Bastian Lange, Ares Kalandides

Birgit Stöber, Inga Wellmann (Hg.)

GoVernange der

Kreativwirtschaft

Diagnosen und Handlungsoptionen

[transcript] Urbanstudies 
Bastian Lange, Ares Kalandides,

Birgit Stöber, Inga Wellmann (Hg.)

Governance der Kreativwirtschaft 

Bastian Lange, Ares Kalandides, Birgit Stöber, Inga Wellmann (Hg.) Governance der Kreativwirtschaft

Diagnosen und Handlungsoptionen 


\section{(9) $(1) \Theta$}

Dieses Werk ist lizenziert unter der Creative Commons AttributionNonCommercial-NoDerivs 4.o Lizenz (BY-NC-ND). Diese Lizenz erlaubt die private Nutzung, gestattet aber keine Bearbeitung und keine kommerzielle Nutzung. Weitere Informationen finden Sie unter https://creativecommons.org/licenses/by-nc-nd/4.o/deed.de/. Um Genehmigungen für Adaptionen, Übersetzungen, Derivate oder Wiederverwendung zu kommerziellen Zwecken einzuholen, wenden Sie sich bitte an rights@transcript-verlag.de

\section{(C) 2009 transcript Verlag, Bielefeld}

Die Verwertung der Texte und Bilder ist ohne Zustimmung des Verlages urheberrechtswidrig und strafbar. Das gilt auch für Vervielfältigungen, Übersetzungen, Mikroverfilmungen und für die Verarbeitung mit elektronischen Systemen.

\section{Bibliografische Information der Deutschen Nationalbibliothek}

Die Deutsche Nationalbibliothek verzeichnet diese Publikation in der Deutschen Nationalbibliografie; detaillierte bibliografische Daten sind im Internet über http://dnb.d-nb.de abrufbar.

Umschlaggestaltung: Kordula Röckenhaus, Bielefeld Lektorat: Anna Stüler, trojan books

Satz: Katja Manz, Leipzig

Druck: Majuskel Medienproduktion GmbH, Wetzlar

Print-ISBN 978-3-89942-996-1

PDF-ISBN 978-3-8394-0996-1

Gedruckt auf alterungsbeständigem Papier mit chlorfrei gebleichtem Zellstoff.

Besuchen Sie uns im Internet: http://www.transcript-verlag.de Bitte fordern Sie unser Gesamtverzeichnis und andere Broschüren an unter: info@transcript-verlag.de 
INHALT

\section{Einleitung}

Fragmentierte Ordnungen

BASTIAN LANGE, ARES KALANDIDES, BIRGIT STÖBER, INGA WELLMANN

Kreativwirtschaft und strategische Stadtentwicklung

KLAUS R. KUNZMANN

Governance und Netzwerke in der Kreativwirtschaft

BASTIAN LANGE

Kreativwirtschaft und Metropolregionen -

Konturen einer systemischen Steuerung

ALAIN THIERSTEIN, AGNES FÖRSTER, STEFAN LÜTHI

Alltagszustand „Risikobereitschaft“"?

Ethnografisch-kulturwissenschaftliches Wissen über Risiko

in der Kreativwirtschaft

ALEXA FÄRBER

Kritik der Kreativindustrien

103

SEBASTIAN OLMA

Neue Arbeitsformen

Reflections on Precarious Work in the Cultural Sector 123

ANGELA MCROBBIE

Allein oder gemeinsam?

Selbständigkeit in der Wiener Kreativwirtschaft

HUBERT EICHMANN, STEFAN LEITNER-SIDL 
„Neue Orte für neues Arbeiten“: Co-working Spaces

BASTIAN LANGE, INGA WELLMANN

Kompetenzen

Kompetenz

155

HARALD A. MIEG

Popkulturförderung in Mannheim

169

SEBASTIAN DRESEL

Design Reaktor Berlin: Ingredienzien einer Prozessgestaltung

177

JUDITH SENG

Neue Institutionen

Schnittstellenkulturen - Hybride Akteure,

Patchworkökonomien, intermediäre Institutionen

183

INGA WELLMANN

Hybrid Space Lab (Amsterdam/Berlin)

199

ELIZABETH SIKIARIDI, FRANS VOGELAAR, INGA WELLMANN

Imagineering

Narrating Urban Entrepreneurship:

A Matter of Imagineering?

CHRIS STEYAERT, TIMON BEYES

Kreatives Singapur

223

BIRGIT STÖBER, CAN SENG OOI

\section{Branding/Marketing}

Orte, Städte und Kreativökonomien als Brand 229

BIRGIT STÖBER, ARES KALANDIDES

Die Bedeutung der Kreativwirtschaft

für die Außenwerbung des Landes Berlin

BJÖRN BÖHNING 


\section{Urban Governance}

Der lokale Staat als Akteur

im Feld kreativer Nischenökonomien

HANS-JOACHIM BÜRKNER

Kultur und Kreativität als Indikatoren

des stadtentwicklungspolitischen Zeitgeistes?

Vom Wandel der Entwicklungsstrategien

der Internationalen Bauausstellung

IBA Emscher Park und IBA Hamburg

261

CONSTANZE GÜNTHER, ACHIM PROSSEK

\section{The Future}

The Challenge of Governance

in the Creative and Cultural Industries

ANDY C. PRATT

\section{Creative Governance}

Reflections on a Governance Model for Creative Industries

289

PERNILLE ASKERUD

\section{Next City}

Just Say Yes: Anmerkungen zur Governance von X-Cities

305

BERNHARD KRUSCHE

\section{Fazit}

Diagnosen, Handlungsoptionen sowie zehn abschließende

Thesen zur Governance der Kreativwirtschaft

BASTIAN LANGE, ARES KALANDIDES, BIRGIT STÖBER, INGA WELLMANN 

Einleitung 



\section{Fragmentierte Ordnungen}

BASTIAN LANGE, ARES KalANDIDES, BIRGIT StÖBER, INGA WELLMANN

Ist die Kreativwirtschaft steuerbar und wenn ja, wie und durch wen? Wie lässt sich Kreativität unterstützen, welche Infrastrukturen benötigen Erwerbstätige in den Bereichen Kunst und Kultur, und inwiefern ist der Begriff Governance anwendbar für die Kultur- und Kreativwirtschaft? ${ }^{1}$ Diese Fragen haben die Herausgeber der vorliegenden Anthologie dazu veranlasst, Praktiker, Wissenschaftler und Kreative einzuladen, ihre Erfahrungen, Erwartungen und Einschätzungen $\mathrm{zu}$ formulieren und hier erstmalig vorzustellen.

Die Kreativwirtschaft als Gesamtbranche wird in Deutschland erst seit vergleichsweise kurzer Zeit aktiv auf Bundesebene wahrgenommen. ${ }^{2}$ So widmet der Abschlussbericht, den die Enquête-Kommission „Kultur in Deutschland“ im Dezember 2007 nach vierjähriger Arbeit dem Bundestag überreichte, der Kreativwirtschaft ein gesondertes Kapitel und räumt ihr eine wichtige wirtschaftliche Bedeutung ein. Bereits im Frühjahr 2007 wurde das Thema erstmals im Rahmen einer eigenen Plenardebatte im Bundestag verhandelt, dabei reichten vier von fünf Fraktionen Anträge zur Förderung der Kulturwirtschaft ein. Ein halbes Jahr später wurde das Konzept für eine „Initiative Kultur- und Kreativwirtschaft" der Bundesregierung unter Leitung des Bundesministeriums für Wirtschaft und Technologie und in Kooperation mit anderen Bundesmi-

1 Wenn im Folgenden von „Kreativwirtschaft“ die Rede ist, dann beziehen wir uns auf die seit 2008 durch die Kultur- und Wirtschaftsministerkonferenz im Bund verabschiedete Definition und Abgrenzung der Kultur- und Kreativwirtschaft (BMWi 2009).

2 Auf Bundesländerebene wird die Allianz von Kultur und Wirtschaft bereits seit den frühen 1990er Jahren beachtet; Vorreiter war hier das Land Nordrhein-Westfalen. 
nisterien vorgestellt. Seit Ende 2008 werden Branchenhearings veranstaltet, konkrete Ergebnisse werden 2009 erwartet.

Trotz großer politischer Zustimmung gibt es kontroverse Diskussionen insbesondere im Bezug auf Förderstrategien. Doch war für den bis Februar 2009 amtierenden Bundeswirtschaftsminister Michael Glos die Bedeutung der Kreativwirtschaft eindeutig: „Wir haben eine bedeutende Stahlindustrie, aber keiner weiß, dass die Kreativwirtschaft inzwischen fast zehn Mal so viele Menschen beschäftigt wie die Stahlindustrie.“3

Wie kam es dazu, dass kreativ bewertete Wirtschaftszweige ohne vergleichbare finanzielle Subventionen der öffentlichen Hand, wie sie die Stahl-, Chemie- oder Energiewirtschaft seit Jahrzehnten erhalten, einen zentralen Wachstums- und Beschäftigungsmarkt im Bund ausmacht und zunehmend gesellschaftliche Relevanz gewinnen? Welcher Wirtschaftskomplex geht mit dem Leitbegriff Kreativwirtschaft einher und wer repräsentiert ihn?

Aus dieser ersten Fragestellung lässt sich sogleich eine zweite ableiten: Wie ist in diese vielschichtige und heterogene Ökonomien unterstützend einzugreifen, ohne ihre höchst individuelle Funktionalität, ihr Autonomiestreben und ihren gesellschaftlichen sowie kulturpolitischen Mehrwert zu beeinträchtigen? Ist dieses Steuerungsparadoxon aufzulösen, und wenn ja, wie?

Diese Fragen verbinden sich zu einer übergeordneten Leitfrage für diese Anthologie: Ist die Kreativwirtschaft steuerbar und wenn ja, wie und durch wen? Universelle Erklärungsmuster, wie sich diese kreativen Ökonomien formieren, versagen ebenso wie die Suche nach konkreten Rezepturen, mit deren Hilfe Fachadministrationen, Investoren aber auch Kulturpolitiker erfolgreich eingreifen könnten. Sowohl das Verstehen der Formierung der Kreativmärkte erscheint von zentraler Bedeutung zu sein, als auch die sich daraus ableitenden Steuerungspraktiken.

Dass wir es nicht mit versteckten Märkten oder einem unsichtbaren Handlungsfeld zu tun haben, zeigen diverse statistische Berichte, häufig in Form von „Kulturwirtschaftsberichten“, die mittlerweile in vielen deutschen Städten (und sogar auch Stadtteilen) publiziert werden. ${ }^{4}$ Ad-

3 Rede des Bundesministers für Wirtschaft und Technologie Michael Glos, MdB, anlässlich der Eröffnung der Popkomm 2007, am 19.9.2007 in Berlin.

4 So gab das Ministerium für Wirtschaft, Mittelstand und Technologie des Landes Nordrhein-Westfalen 1992 den ersten Kulturwirtschaftsbericht sowie die so genannte Essener Erklärung heraus; mittlerweile ist der fünfte Kulturwirtschafsbericht in Nordrhein-Westfalen erschienen. Seither nahmen auch andere deutsche Bundesländer diese Art der Inventarisierung ihres kulturellen und kreativen Bestandes bzw. Potenzials auf und signali- 
ministrative Initiativen und privatwirtschaftlich organisierte Plattformen leisten ihresgleichen, um die gesellschaftliche Akzeptanz und Legitimation einer zunehmenden Zahl von Arbeits- und Lebensentwürfen in diesen Ökonomien zu erlangen. Uns erscheint es sinnvoll, im Zuge der Frage nach der Governability der Kreativwirtschaft, also der Steuerbarkeit, immer auch nach zentralen Elemente der Formierungslogik dieses jungen Handlungsfeldes zu fragen, d. h. nach dem strukturell Neuen dieser Ökonomien.

\section{Governance der Kreativwirtschaft}

Das Konzept und den Begriff Governance auf das Handlungsfeld der Kreativwirtschaft anzuwenden, ist erklärungsbedürftig und stellt eine komplexe Herausforderung dar. Erklärungsbedürftig, weil beide Konzepte relativ jung sind und sich somit nicht durch belastbare Definitionen ausweisen. So ist der Begriff Governance gegenwärtig in den $\mathrm{Hu}-$ man-, Rechts- und Wirtschaftswissenschaften ein Leitbegriff. Die Leitfunktion erhält der Begriff dadurch, dass der hierarchische, zentralistische und dirigistische Charakter traditioneller staatlicher Steuerungsformen durch neue, dezentrale, netzwerkartige Formen der Kontextsteuerung ergänzt und teilweise abgelöst wird (Brand 2004). Besonders in der raumbezogenen Governance-Debatte wurde der Begriff weiter entgrenzt (Haus et al. 2005) und auf Fragen der Verhandlung von raumrelevanten Ressourcen in Metropolregionen übertragen.

Ähnlich vage ist die Definition des Begriffs Kreativwirtschaft. Er erfreut sich zwar seit dem Jahr 2008 einer „offiziellen“ Definition durch die Wirtschafts- und Kultusministerkonferenz, gleichwohl wird die Verwendung der Begriffe Kreativität und Kreativwirtschaft von der Fachöffentlichkeit weiterhin kritisch beäugt. Nicht zuletzt wird diese Debatte durch den kaum fixierbaren Leitbegriff Kreativität immer wieder neu entfacht, weshalb „Rückrufaktionen“ gebotener denn je erscheinen (Althans et al. 2008).

So gesehen wagt die vorliegende Anthologie just in dem Moment, in dem langwierige Definitionsversuche am Konzept Governance und angemessene statistische Abgrenzung des Branchenbegriffs Kreativwirtschaft vollzogen wurden, eine weitere Entgrenzung: Sie prüft die Anwendung des Begriffs Governance auf aktuelle Formierungslogiken von

sierten damit die Wichtigkeit des Themas Kultur- bzw. Kreativwirtschaft oder auch Creative Industries. 
Marktteilnehmern, Märkten und momentane Förderpraxen für die Kreativwirtschaft.

Dabei wird mit dieser Anthologie ein weiterer Schritt in der Analyse des Handlungsfelds Kreativwirtschaft vollzogen, in dem ihre Formierungspraxis untersucht wird. Aufgrund des hybriden und schnell wandelnden Charakters dieser Märkte überprüfen wir die Schnittstellen zu anderen, auch etablierten Dienstleistungssegmenten. Dadurch kann eine erste Erweiterung des eng definierten Begriffs Kreativwirtschaft erfolgen, hin zum strategischen Begriff von Kreativökonomie, den z. B. das Land Nordrhein-Westfalen seit 2008 verwendet.

Zusätzlich bemühen sich die Autoren dieses Buches darum, die Aktivitäten der Kreativwirtschaft im Kontext ihrer jeweiligen sozialräumlichen Bedingungen sowie ihren spezifischen Funktionsprinzipien gerecht zu werden.

Abgrenzungsbestrebungen und Definitionsabsichten auf fachöffentlicher Seite führen leicht dazu, Gegenstände durch Beschreibungen eher zu bereinigen, als ihre auch mögliche paradoxe Komplexität und irritierende Heterogenität zuzulassen. Dies erscheint aber gerade für dieses Spannungsfeld notwendig, da weder vollendetes Wissen um die Formierungspraxis der Kreativwirtschaft vorliegt, noch Governance-Verständnisse für dieses junge Handlungsfeld. Vor diesem Hintergrund erhält die vorliegende Anthologie, deren Vorhaben es ist, die komplexen Kontexte der Kreativwirtschaft aufzuspüren und zu diskutieren, hohe analytische sowie praktische Relevanz.

Die Anwendung der Dimension Governance auf die der Kreativwirtschaft ist ein neues Unterfangen, das erst seit wenigen Jahren als Forschungs- und Handlungsfeld in Augenschein genommen wird. Im Kern der Debatte stehen zweifelsohne übergeordnete Fragen, nämlich die nach der Autonomie von Kunst und Kultur sowie dem gesellschaftlichen Stellenwert ihrer Hauptprotagonisten, den Künstlern und Kulturschaffenden. Daran knüpft sich die Frage nach ihrer Selbstbestimmtheit im Spannungsfeld zwischen existenzsichernder Arbeit, individuellen Verwirklichungsoptionen und gesellschaftlicher Akzeptanz.

Im Folgenden wird ausgelotet, wie Handlungs- und Gestaltungsfreiheiten mit Markterfordernissen abgestimmt werden können, wie die Einbindung in soziale Vertrauensnetze mit Marktmechanismen und damit verbundenen Konkurrenzsituationen abgewogen werden und wie sich die Notwendigkeit individueller Verwirklichung in Beziehung zu kollektiven Interessen darstellen. Diese Binnensicht erklärt wesentliche Dimensionen der Formierungspraxis der Kreativwirtschaft. Sie wird im weiteren Verlauf als Self-Governance (Kooiman 2003) konzeptiona- 
lisiert, um am Fall der Kreativwirtschaft einen systematischen Brückschlag zu Co-Governance- sowie hierarchischen Governance-Formen zu eröffnen.

\section{Governance}

Das Wort Governance ist als Alltagsbegriff im englischen Sprachraum gebräuchlich, mit dem ,the act or manner of governing“ bzw. „the office or function of governing“ bezeichnet wird (Oxford Dictionary). In Anlehnung an diese alltägliche Bedeutung wurde der Begriff aber als Bezeichnung für eine wissenschaftliche Perspektive herangezogen.

Ein Konzept von Governance zielt nicht nur auf die Beschreibung der Handlungskoordination verschiedener Akteure, sondern vielmehr auf die unterschiedlichen Formen und Mechanismen dieser Koordination. Unter Formen versteht Benz (2007) Strukturen der kommunikativen Interaktion, die aus einem dauerhaften Zusammenwirken folgen oder durch Regeln institutionalisiert werden können. Prozessverläufe, die sich begründet im Rahmen dieser Interaktionsformen ergeben, werden demzufolge als Mechanismen bezeichnet. Daraus lassen sich die Betrachtungsmerkmale der Governance-Perspektive ableiten.

Daneben existieren weitere Anwendungsmöglichkeiten des Governance-Begriffs, die weniger analytischer denn normativer Natur sind: Good Governance (Kötschau 2008), Globale Governance (Gruber 2008) oder Corporate Governance (Sick 2008), die letztlich auf der Annahme „guten“ Regierens und Führens aufbauen. Anders als diese Konzepte steht das eingangs adressierte Konzept von Governance für einen analytischen Werkzeugkasten zur Beschreibung und zum Verstehen kollektiven Handelns (Benz 2007).

Während sich die Governance-Debatte in Deutschland lange Zeit am staatstheoretischen Begriff Steuerung abgearbeitet hat und somit Governance im staats- und demokratietheoretischen Verständnis bearbeitet wurde, erweitert die internationale Debatte das Governance-Konzept. Insbesondere Kooiman (2003) schlägt verschiedene Modes of Governance vor, die hier kurz skizziert werden. Diese Perspektive ist im Kern eine interaktionsbasierte und dadurch im Wesentlichen eine soziologische, wodurch sie in der Auslegung von Kooiman weitaus stärker durch gesellschaftliche Diagnosen informiert wird. So stehen Fragen der sozialen Differenz, der gesellschaftlichen Komplexität und den daraus erwachsenen Dynamiken im Mittelpunkt seines heuristischen Rahmens. Folgende Modes of Governance werden näher betrachtet: 


\section{Self-Governance}

Self-Governance verweist auf kommunikative Formen der Selbstorganisation und Selbststeuerung, die gerade in so genannten Kreativszenen und Kreativmilieus (Lange 2007) deutlich zu erkennen sind. Diese Gebilde weisen keine hierarchische Macht- und keine formale Repräsentationsstruktur auf. Als regelrecht autopoietische Teilsysteme sind sie durch ihre wirkmächtigen und abgeschotteten Binnenlogiken von außen für Uneingeweihte schwer zugängliche Kommuniktionsfelder. Umgangssprachlich verdeutlicht der Begriff Künstlerzirkel diese Praxis. Die verschlossenen Kommunikationsnetze sind für die Teilnehmer essentiell: sie stellen einen geschützten Rahmen dar, um z. B. noch unfertige Produkte hinsichtlich ihrer Wirkung auf ein Publikum testen können.

Aufgrund dieser oftmals kontaktdichten Sphären ist es aus der Sicht einer Förderperspektive schwierig, in diese informellen Netzwerke stabilisierend und unterstützend einzugreifen. Fragen der Interessenvertretung, der Abstimmung und der Selbstbeschreibung sind entweder schwach entwickelt, spielen mit der Rolle des Außenseiters, oder schotten sich gegen andere Logiken zur Wahrung ihrer Individualität $a b$ (Kooiman 2003: 82). Gleichwohl sind diese Binnenlogiken auch immer als fruchtbare Basis intensiver Selbststeuerungsmechanismen anzusprechen: Auf dieser Grundlage können auch formalere und komplexere Steuerungsformen verhandelt werden, da in diesen Selbststeuerungsformen das Wissen um Formierungspraktiken zwischen den Akteuren vorhanden und erprobt sind. Finden Akteure verschiedener Teilsysteme z. B. in Form von Netzwerken (Messen, Ausstellungen usw.) zusammen, so treffen nicht nur verschiedene Selbststeuerungslogiken aufeinander, vielmehr weist die Steuerungspraxis des Netzwerks notgedrungen eine eigene Governance-Praxis auf, für die zunächst kein vorhandenes Wissen um Spiel- und Verfahrensregeln zwischen unterschiedlichen Netzwerksphären vorliegt.

Heterogene Steuerungslogiken sind sodann im Sinne von Kooiman zunächst Formen der kommunikativen Störung, die sich zwischen autopoietischen Teilsystemen ergeben. Sie sind dabei per se zunächst unorganisiert, fluide und informell. Explizites Wissen um Formalisierung dieser Störungszone ist nicht vorhanden, so dass Netzwerk- und Milieuwissen einen übergeordneten Stellenwert erhalten, um zielführende Interaktion überhaupt aufrecht zu erhalten. Dieser Blick auf die Steuerungsverhältnisse erscheint gerade für junge und emergierende Märkte, wie sie zu einem großen Teil in den Kreativbranchen vorzufinden sind, relevant, da auch etablierte Interessenvertreter von z. B. der Film- und Kunstbranche oftmals eine große kommunikative und lebensweltliche 
Distanz zur Basis aufweisen, oder mitunter einer schmalen, aber nicht repräsentativen Klientel das Wort reden.

Gleichwohl sind diese kommunikativen Störungszonen zwischen Teilsystemen auch immer die Grundlage für neue Lösungswege, dafür, wie die Störungen beseitigt und wie passende und erfolgreiche Verfahrensregeln der Kollaboration und Kooperation etabliert werden können. An diesen Schnittstellen und kommunikativen Bruchzonen begegnen die zahlreichen mikrokollektiven Produktionsnetzwerke der Kreativwirtschaft den etablierten und zugleich strukturell machtvollen und repräsentativen Systemen der Gesellschaft.

An diesen Stellen artikuliert sich das „Eigene“, gewissermaßen der genetische Code dieser Mikrokollektive, zunächst in Form von neuen Sprech- und Sichtweisen über neue Produkte sowie neue Verfahrensund Produktionsweisen. Darüber hinaus verweist es eben nicht auf gleichberechtigte Interaktionspartner sondern auf hochgradig unterschiedliche Kommunikationsregeln und Interaktionspraktiken. Als Konsequenz dieser kommunikativen Differenz erwächst eine neue, wenn auch noch kleine, professionelle Gruppe von Schnittstellenakteuren (siehe Wellmann in diesem Band), die nicht nur Verbindungen zwischen getrennten Welten herstellen, sondern auch in der Lage sind zu erläutern, worin diese Trennung liegt und wie ein Mehrwert aus einer kommunikativen Interaktion folgen könnte. Gelingen Interaktionen zwischen verschiedenen Teilsystemen, spricht man von Co-Governance.

\section{Co-Governance}

Das Vorzeichen „Co“ verweist auf verschiedene Formen der Kooperation, der Kollaboration sowie der Kommunikation, um mit und gegenüber Anderen spezifische Interessen zu vertreten. Dazu gehören auch soziale Netzwerke, die als eine Form des Co-Governance anzusprechen sind.

Die kommunikative Dimension setzt zum einen die Fähigkeit zur Verständigung voraus und bewertet Kommunikation als eine Form von Governance, da sie gegenüber formalisierten Verfahren (Bürokratie) oder Wettbewerbsverfahren (Markt) adäquater, weil situationsbezogener ist und zudem als dialogische Form neutraler und reflexiver mit struktureller Macht umzugehen weiß. $\mathrm{Zu}$ der Form Co-Governance gehören auch die lange Zeit im Scheinwerferlicht der Kommunalpolitik stehenden Public-Private-Partnerships (PPP).

Die Dimension Vertrauen spielt eine wesentliche Bedingung von Interaktion und verweist auf die Labilität temporärer Kooperationspraxis hin. Gerade der öffentliche Sektor ist durch die Verbindung und die Al- 
lianz mit Marktteilnehmern oftmals Legitimationsproblemen ausgesetzt. Dies zeigt sich z. B. in der Diskussion um den Stellenwert und die Relevanz der öffentlichen Kulturförderung im Verhältnis zu Marktbedingungen.

Als eine zentrale Kategorie von Co-Governance-Formen sind auch soziale Netzwerke zwischen Firmen, Non-Profit-Organisationen (NGO) oder öffentlichen Einrichtungen als separate Verhandlungssphären anzusprechen. Weitaus relevanter für Governance-Perspektiven sind aber Interaktionsformen zwischen der Sphäre Gesellschaft und Markt sowie Zivilgesellschaft und Staat. Co-Governing hebt sich von situativen Formen der Self-Governance in der Gestalt ab, als dass Co-GovernanceFormen systematische Kombinationen von verschiedenen Interessen darstellen. Auch diese können situativ und temporär seien, müssen sich also gegenüber Self-Governance-Formen nicht durch einen höheren Formalisierungsgrad der Interaktionsbeziehungen auszeichnen.

\section{Hierarchische Governance}

Hierarchische Governance mit Top-down organisierten, mechanistischen Formen des Government gleichzusetzen, würde ausblenden, dass hierarchische Governancen-Formen auf die Frage der Steuerung sowie der Steuerungsrichtung abzielen. Im Vordergrund steht dabei die Frage von Kontrolle über die Steuerungsrichtung, was angesichts wachsender Komplexität in den jeweiligen Steuerungskontexten schwieriger wird. Nicht zuletzt zeigt die seit dem Herbst 2008 andauernde Finanzkrise, dass Selbstkontrolle nicht nur in Managementsegmenten schwer zu realisieren ist.

Auch seitens der öffentlichen Hand und seiner staatlichen Aufsichtsbehörden haben wichtige Kontrollfunktionen nicht gegriffen. Dieses Beispiel verdeutlicht auch die Reichweite des Kontrollanspruchs des Staats im Nach- und Fortgang der Finanzkrise, in der er als Kontrolleur in Marktkontexten wirken will, um die eingeleiteten Maßnahmen zu überprüfen. Daran zeigt sich auch, dass nach wie vor von starken steuernden staatlichen Rahmungen auszugehen ist, mitunter sind diese im Zuge der Europäisierung und der Neuformulierung von EU-Rechten, EU-Richtlinien und EU-Praktiken eher noch umfangreicher und komplexer geworden, als dies im Zuge von gesellschaftskritischen sowie neoliberalen Diskursen wahrgenommen wird.

Übertragen auf das Handlungsfeld der Kreativwirtschaft in Deutschland bedeutet dies, dass insbesondere der Staat im Bereich der Kultursowie Wirtschaftspolitik nach wie vor eine eindeutig normative Position einnimmt und mit seinen Definitionsvorgaben stark die Wahrnehmung 
und Artikulation dieses Feldes beeinflusst. Das wirkte sich in dem jüngsten Definitionsansatz der Kultus- und Wirtschaftsministerkonferenz im Frühjahr 2008 aus, in der eine statistische Abgrenzung des Feldes gewissermaßen Top-down implementiert wurde.

\section{Fazit}

Die vorangegangene Darstellung dreier unterschiedlicher Modes of Governance hat das Ziel, nicht nur den Aspekt Self-Governance für das Handlungsfeld Kreativwirtschaft zu stärken, sondern ebenso den Aspekt der Schnittstellensteuerung (Co-Governance), wie er sich zwischen etablierten und nicht-etablierten Policy-Machern zu erkennen gibt.

Ebenso relevant erscheint aber auch der nach wie vor starke Staat als eine Entität, die auf dem Weg der Aktivierung und der Ermöglichung auch daran interessiert ist, kulturelle Güter und die von ihm geleisteten Investitionen zu kontrollieren. Gleichzeitig hat sich das Konzept Governance vor dem Hintergrund dieser drei Modes of Governance von seiner demokratie- und staatstheoretischen Ausrichtung gelöst und ist als Konzept zu einem umfassenden Instrumentarium avanciert, das die Analyse von Interaktionsbeziehungen innerhalb von komplexen Kommunikations- und Handlungsfeldern ermöglicht.

Ziel der vorliegenden Anthologie ist es also, aus vielen unterschiedlichsten Perspektiven, die Schnittstellen zwischen der Kreativwirtschaft und Governance-Formen zu hinterfragen und daraus Konsequenzen abzuleiten.

\section{Das Konzept Kreativwirtschaft: Was ist daran neu?}

Der Begriff Kreativwirtschaft skizziert nicht nur neue, als kreativ bewertete Ökonomien, er steht auch für ein neues gesellschaftliches Verhältnis zwischen Kultur und Ökonomie. Arbeit und Markt organisieren sich in und durch soziale Netzwerke, etablierte Sicherheiten werden sukzessive entwertet und formieren sich entlang neuer Wissens- und Kompetenzformen. Neue Geographien geben sich zu erkennen, aus dem Blick gefallene und deindustrialisierte Orte greifen nach dem hoffnungsvollen Zauber der „kreativen Stadt“, um einen Neuanfang einzuleiten. Neue Macher und Schnittstellenakteure, sogennannte Culturepreneurs, bestimmen als eine Art Raumpioniere mit ihrer Improvisationskompetenz die brachliegenden Zwischenräume mit neuen Produkten und ungeplanten Vermarktungsweisen. Sie inszenieren sich selbst als Marke und re- 
präsentieren einen neuen Unternehmertypus, der für die etablierten Institutionen ungewohnt ist.

Innerhalb der Kreativwirtschaft verläuft aber eine Bruchlinie: Auf der einen Seite stehen traditionelle Kompetenzzentren der Kreativwirtschaft, die mitunter seit mehreren Jahrzehnten am Markt operieren. So stellt z. B. die Musikinstrumentenproduktion mit ihren kleinteiligen Manufakturstrukturen in Randlagen des Freistaats Sachsens ein wesentliches Schwergewicht der sächsischen Kreativwirtschaft dar. Auf der anderen Seite stehen neue, hochdynamische standortungebundene Märkte, v. a. der Software und Games Industrie.

Maßgebliche Triebfeder der Funktionalität dieser neuen Ökonomien sind die abnehmende Halbwertzeit der Gültigkeit von marktrelevantem Wissen, hoher Innovationsdruck und schnell wechselnde Projektkulturen. Diese meistens wenig technikaffinen Ökonomien ermöglichen, so eine positive Lesart, individuelle Selbstverwirklichung, sozialen Statusgewinn, aber auch, so die Kritik, nur sehr selektive ökonomische Existenzsicherung (Manske 2008). Dadurch werden Berufe, Arbeitsstrukturen, Wohnformen und Kommunikationsbedarfe grundsätzlich neu justiert.

Diese Märkte der Kreativwirtschaft werden von zahlreichen Befürwortern und Zukunftsforschern als eine Blaupause für die Neuformierung und Konstituierung eines als zukunftsträchtig bewerteten kreativen Zeitalters beschrieben. Die Bewältigung des Wandels hin zu einer durch Kreativität bestimmten Arbeits- und Lebenswelt geht aber mit einem zunehmenden Steuerungsbedarf der Städte einher, wobei die Steuerungswirkung gleichzeitig tendenziell abnimmt.

Bei der Neuentwicklung von Infrastrukturen, Wohn- und Arbeitsformen stellt sich daher das Problem, dass die oft nach wie vor traditionellen Instrumente und Lenkungssysteme nur wenig auf die Arbeits- und Interaktionsbedingungen der Kreativwirtschaft eingerichtet sind. Vor diesem Hintergrund kann die nachhaltige Wirkung der urbanen Steuerungspraxis nur schwach sein, woraus wir einen Bedarf nach neuen Steuerungsformen zwischen Staat, Privatwirtschaft und intermediären Akteuren ableiten.

\section{Die Akteure der Kreativwirtschaft: Arbeit - Netzwerk - Governance}

Der Wandel der Arbeit in der Kreativwirtschaft ist komplex und artikuliert neue Erwartungshaltungen an Individuen: Diese oszillieren im Spannungsfeld glamouröser Berufsbilder und Hoffnungen auf gesell- 
schaftlichen Statusgewinn. Viele neue Berufsfelder sind kaum etabliert, übergeordnete Regulationsinstrumente fehlen weitestgehend, so dass Technologien des Selbstmanagements dominieren, um eine professionelle Existenz in diesem Handlungsfeld einzunehmen. Die hitzige Diskussion um die prekäre Arbeitssituation (Ellmeier 2001; Lorey 2007) übersieht mitunter, dass sich neben den individuellen An- und Aufforderungen zur Selbstorganisation die Arbeit in der Kreativwirtschaft entlang temporär wirksamer Kollektive formiert: während sich die Kreativszene als Digitale Bohème (Friebe/Lobo 2006) beschreibt und dabei - mal ironisch, mal distanzierend - als Avantgarde auftritt, antworteten die Sozial- und Raumwissenschaften mit nüchternen Begriffen wie Projektökologien (Grabher et al. 2004), Communities of Practice (Wenger 1999), Kreativszenen (Lange 2007) oder Wissensmilieus (Matthiesen et al. 2004). Allen ist gemein, dass gewerkschaftlich erkämpfte Freiheits- und Sicherheitsgarantien, die durch übergeordnete Vertretungen aufrecht erhalten und kontrolliert werden, zunächst nur äußerst schwache Wirkung in kreativ- und wissensökonomischen Kontexten haben. Vielmehr zeigen sich unter dem Vorzeichen von Selbstbestimmung neue und bislang wenig erprobte Arbeitskonstellationen, die sich durch ein hohes Maß an Passgenauigkeit an die Bedingungen der Arbeitswelt und der Arbeitsauffassung der Kreativwirtschaft auszeichnen. Flexibilität und Transformationsbereitschaft werden zu Schlüsselkompetenzen.

Gerade junge Unternehmer in der Kreativwirtschaft müssen gegenüber traditionellen (Kultur-)Institutionen, Grossunternehmen und finanzintensiven Kulturproduktionen wie z. B. in der Filmwirtschaft üblich, signifikant anders operieren. Sie müssen ihre Tätigkeiten und ihre Verfahrensweisen sowie gemeinschaftlich-integrierte Projektsteuerungen in einem instabilen Kontext sowie einem jungen und zugleich hochdynamischen Markt verorten. Markteintritt und Aufmerksamkeitsmaßnahmen auf dem Weg der Identitätsbildung sowie der Entwicklung seiner Selbst als Marke müssen neu erfunden werden (Friebe/Lobo 2008; Lange 2008). Die dabei zu beobachtenden Arbeitsformationen und Arbeitsformate, also Netzwerke, temporäre Kollektive usw. lassen sich auch als Teil eines gesellschaftlichen Phänomens ansprechen.

Diese temporären Projektnetze der kulturellen Produzenten sind zwar mitunter durch instabile Finanzierungsgrundlagen bestimmt. Die international ausgerichteten Arbeitsnetze zielen neben der Einlösung eines symbolischen und Autonomiegewinns aber parallel auf den Aufbau eines zweiten Sicherheitsboden: den der sozialen Netzwerke. Diesen Netzwerken kommt eine maßgebliche Rolle zuteil. Es sind ad-hocGebilde, situative Arbeitsformationen, die einen geringen Formalisierungsgrad aufweisen und in denen Vertrauen eine zentrale Bedeutung 
spielt. Ein wesentliches Kennzeichen dieser Arbeitspraxis ist, dass die Akteure symbolische Güter produzieren, deren Bewertung nicht nur monetären Kriterien unterliegt. Zahlreiche erbrachte Leistungen vollziehen sich auf kollegialen und freundschaftlichen Ebenen, weisen also ein hohes Maß an sozialer Verbindlichkeit auf. Diese Netzwerke sind demnach reich an symbolischem und sozialem Kapital, wobei die Hoffnung allgegenwärtig sein mag, dieses Kapital der symbolischen und sozialen Art im bourdieuschen Sinne zu einem Zeitpunkt auch in ökonomisches Kapital umwandeln zu können. Die informellen Dimensionen des Handelns sind ein Kennzeichen für die Art und Weise, wie Markteintritt, Preisbildung und Entlohnung auf breiter Basis neu bewertet werden. Diese Praxis ist nicht neu, sie ist aus Kunstkollektiven ebenso bekannt wie in schwach regulierten Wirtschaftszweigen sowie der informellen Wirtschaft.

Trotz schwieriger Arbeits- und Existenzbedingungen wirken die kulturellen Produzenten der Kreativwirtschaft zunehmend proaktiv an der Erzeugung und der Entwicklung einer eigenen gesellschaftlichen Position. Ihre vernetzten temporären lokal-globalen Arbeitswelten fordern die etablierten Institutionen durch ihre ungewöhnlichen und gewissermaßen ungeordneten Verfahrensweisen heraus. Sie geben dabei fragmentierte Ordnungen zu erkennen. Den etablierten Institutionen fehlt oft noch ein geschickter Umgang mit existierenden gesellschaftlichen Instabilitäten. Sie haben dafür zunächst kein Steuerungs- und Handlungswissen parat. Zur Bewältigung dieses Defizits leistet die Anthologie einen ersten Beitrag.

\section{Kreativwirtschaft als Motor von urbanen Restrukturierungen}

Schon Jahre vor Richard Floridas publizistischem Erfolg erkannten europäische Regionalpolitiker sowie Stadt- und Regionalplaner die Wichtigkeit von Kultur und Kreativität zur Revitalisierung sowie Imageund Standortaufwertung von strukturschwachen Gebieten. Sowohl in Großbritannien, in Sheffield, als auch in Nordrhein-Westfalen wurden in den 1990er Jahren umfassende Aktivitäten zur Umwidmung von altindustriellen Arealen in kulturelle Erlebnislandschaften in Gang gesetzt. Diese Maßnahmen, die Kultur als harten Standortfaktor ins Zentrum stellten, wurden zum Teil von neugegründeten Entwicklungsgesellschaften durchgeführt und von umfangreichen Informations- bzw. Marketingkampagnen begleitet, so dass Kultur und Kreativität zu einem wichtigen 
Bestandteil von Stadt- und Regionalmarketing wurde (siehe Beitrag von Stöber und Kalandides in diesem Buch).

Diese Art der Marketingaktivitäten beschränkte sich jedoch nicht nur auf Städte und Regionen. Auch auf nationaler Ebene werden seit den 1990er Jahren Kampagnen zur Imageaufbesserung und Gewinnung von Aufmerksamkeit der positiven Art initiiert. Eine der bekanntesten, jedoch nicht unbedingt auch erfolgreichsten Unternehmungen dieser Art ist die „Cool Britannia“ Kampagne, die zu Tony Blairs Amtszeit nach der britischen Rinderwahn Krise das Image des Königreiches wiederherstellen sollte. Mit Hilfe dieser Kampagne sollte die britische Nation von ihrem veralteten five o'clock tea image befreit und mit dem neuen Image einer energischen, spannenden und liberalen Nation versehen werden.

Mit Hilfe neuer Governance-Formen wird versucht Nationen, Städte oder Kommunen im globalen Wettbewerb um Investitionen zu positionieren, wobei die Beteiligung an globalen Netzwerkbildungen im Blick behalten wird. Ökonomische Entwicklung in wissensbasierten und kreativen Branchen wird dabei aufgrund ihrer Komplexität als eine Aufgabe begriffen, die eine Vielzahl städtischer Akteure angeht. Urbane Steuerungsprozesse werden daher in zunehmendem Maße durch unterschiedliche Konstellationen von (lokal-)staatlichen, privatunternehmerischen und zivilgesellschaftlichen Akteuren getragen. Die Heterogenität der Akteure wird nachdrücklich als Merkmal partizipativer Steuerungsformen betont (Cars et al. 2002; Healey 2004).

Als private Akteure kommen nicht nur Unternehmen, Quartiersbewohner, lokale Vereine, zivilgesellschaftliche Initiativgruppen usw., sondern vermehrt auch neue Intermediäre (z. B. Unternehmensberater, private Entwicklungsgesellschaften, professionelle Mediatoren) ins Spiel. Auch die Akteure der Kreativwirtschaft, die „Kreativen“ selber, weisen - aufgrund ihrer Anbindung an unterschiedliche Netzwerke und Arbeit in unterschiedlichsten Projektkonstellationen - zunehmend so genannte Schnittstellenkompetenzen auf.

Die Komplexität neuer Ökonomien, die heute eben nicht mehr den gängigen betriebswirtschaftlichen Logiken folgt, sondern vielmehr auf immateriellem Kapital wie Anerkennung, Glaubwürdigkeit und multidisziplinären, sozialen Beziehungsnetzen beruht, machen diese Kompetenzen überlebensnotwendig. Auch die Möglichkeiten, die dem vernetzt arbeitenden Einzelnen durch neue Technologien, respektive Web 2.0, eröffnet werden, verändern die Strukturen der Einflussnahme auf Markt und Politik sowie die Wechselwirkung unterschiedlicher Disziplinen und Akteurskonstellationen. Dynamische Formen der Selbstorganisation treten an die Stelle organisierter Interessensvertretung, erreichen in der 
Regel jedoch nur selten die politischen Entscheidungsträger in der öffentlichen Administration.

Deutlich wird jedoch, dass die besonderen Interaktionskulturen der Kreativwirtschaft auf der städtischen Maßstabsebene für ein strukturelles Paradoxon der Stadtentwicklung sorgen (DeFillippi et al. 2007; Grabher 2004; Lange et al. 2008a; Lange et al. 2008b): Während sich die ökonomischen Akteure soziale Einbettungszusammenhänge im Nahbereich schaffen, nimmt ihre Orientierung an überlokalen Arbeitszusammenhängen und Marktgeschehnissen ebenfalls zu. Die Verbindung zu lokaler Politik und Gesellschaft wird dadurch so distanziert, dass Beteiligungen an Steuerungsprozessen zwar erfolgen, aber nicht mit der erforderlichen Energie vorangetrieben werden. Über die organisationsbezogenen und sozialen Prozesse, die mit der Positionierung der Akteure der Kreativwirtschaft in diesen Governance-Prozessen zusammenhängen, ist derzeit kaum etwas bekannt.

\section{Förderung der Kreativwirtschaft}

Auch wenn die Bedeutung der Kreativwirtschaft in der Bundesrepublik Deutschland erst seit kurzer Zeit in gebündelter Form Gehör findet und ein gezieltes Engagement bislang aussteht, so werden einzelne Teilbranchen der Kreativwirtschaft bereits durch eine Vielzahl von Aktivitäten und Maßnahmen gefördert. $\mathrm{Zu}$ diesen Aktivitäten gehören etwa der Gründerwettbewerb Multimedia, der Designpreis, Messeförderungsmaßnahmen, der Deutsche Musikinstrumentenpreis oder die als öffentlich-private Partnerschaft angelegte Initiative Musik. Insbesondere die Filmwirtschaft erfährt besondere Aufmerksamkeit durch den Bund in Form verschiedener Auszeichnungen (Deutscher Filmpreis, Deutscher Drehbuchpreis, Kinoprogrammpreis) sowie Projektfilmförderungen finanziert durch den Deutschen Filmförderfonds.

Jedoch richtet sich die Förderpolitik des Bundes, genauer des Beauftragten der Bundesregierung für Kultur und Medien (BKM), in erster Linie auf die Entwicklung von Kunst und Kultur und der Unterstützung entsprechender Rahmenbedingungen und weniger auf die direkte Förderung von Unternehmen der Kreativwirtschaft. Im Gegenzug ermutigt das Bundesministerium für Wirtschaft und Technologie die Unternehmen und freiberuflich Tätigen der Kreativwirtschaft zwar, bestehende Wirtschaftsförderangebote in Anspruch zu nehmen, jedoch zeigt sich bei näherem Hinsehen, dass nur ein Bruchteil der Kreativwirtschaftsakteure die erforderlichen Bedingungen erfüllt. Dieser in Deutschland noch etwas unbeholfen anmutende Spagat zwischen Kultur und Wirtschaft ist 
kennzeichnend für die Förderlandschaft der Kreativwirtschaft. Aufgrund der starken Wechselwirkung mit öffentlich finanzierter Kultur stellt die Kreativwirtschaft die bisherigen ressortpolitischen Abgrenzungen massiv in Frage. Kulturpolitik muss sich dem Markt, den veränderten Realitäten der in komplexen, sektorenübergreifenden Netzwerken agierenden Kulturschaffenden stellen. Gleichzeitig gewinnen wirtschaftspolitische Förderinstrumente an Relevanz für Kultureinrichtungen.

Auch auf Länderebene haben die Wirtschaftsministerien in der Regel klassische Wirtschaftsförderprogramme zusätzlich für die Kreativwirtschaft geöffnet. Doch sind diese, so das Ergebnis der Enquete Kommission des Deutschen Bundestages im Jahr 2008, nicht auf die Kleinteiligkeit, die besonderen Akteurstypologien sowie die durch Unikate und Symbolwert gekennzeichnete Produktpalette der Kreativwirtschaft ausgerichtet. Dies führt dazu, dass die angebotenen Fördermaßnahmen kaum oder nur mit unverhältnismäßig großem Aufwand ausgeschöpft werden. Umfragen der Enquete-Kommission bei den Kulturministerien der Länder ergaben zudem, dass auch die öffentlichen Kulturförderangebote durch privatwirtschaftlich agierende Kulturschaffende kaum genutzt werden können.

Eine Zusammenarbeit oder Abstimmung zwischen Wirtschafts- und Kulturministerien hat bisher nur in Ausnahmefällen stattgefunden. Die wesensimmanente Hybridität der Kreativwirtschaftsunternehmen und -projekte führt automatisch zu einem Herausfallen aus sämtlichen Förderkategorien, unabhängig von Größe oder volkswirtschaftlicher Bedeutung. Besonders benachteiligt sind jedoch Kleinstunternehmer, die den hohen bürokratischen (Begründungs-)Aufwand, der oft mit öffentlicher Förderung einhergeht, kaum bewältigen können. Auch fehlt es an Vermittlungsinstanzen zwischen den beiden wenig kompatiblen Denk- und Herangehensweisen öffentlicher Mittelvergabe und freien kreativen Milieus.

Eines der wichtigsten Handlungsfelder ist eine auf die Kleinteiligkeit, Kurzfristigkeit und Dynamik vieler kreativer Projektverläufe ausgerichtete Kapitalmittelbeschaffung. Nach dem Vorbild der Mikrokreditvergabe, die ihren Ursprung in der von dem bangladeschischen Wirtschaftwissenschaftler und Friedensnobelpreisträger Muhammad Yunus begründeten Grameen Bank hat, reagieren nun auch in Deutschland einige Kreditinstitute mit der Einführung entsprechender Kreativwirtschaftsfonds. Damit soll dem besonderen Bedarf kreativer Unternehmen nach einer flexiblen Vergabe von (Kleinst-)Krediten und Darlehen Tribut gezollt werden. Als Beispiel sei hier die Investitionsbank Berlin Brandenburg (IBB) angeführt, die im Dezember 2007 einen Fonds für Kreativwirtschaft und Technologieunternehmen ins Leben rief, der sich 
speziell an Unternehmen aus dem Kultur- und Medienbereich, wie etwa Film-, Design- aber auch Computerspielfirmen richtet. Von einer selbstverständlichen Praxis im deutschen Kreditwesen kann hier jedoch noch nicht die Rede sein.

Parallel zu direktionalen Förderinstrumenten stellt gerade die Kontextförderung einen nicht unerheblichen Faktor für Wachstum der Kreativwirtschaft dar und gewinnt (wie sich im Verlauf dieses Buches zeigen wird) gerade im Hinblick auf die Frage nach den Steuerungsoptionen für die Kreativwirtschaft an Bedeutung. Auch wenn sich verschiedene Maßnahmen in der Vergangenheit bereits dem kulturtouristischen Marketing, der Clusterförderung, der Raumerschließung durch Umnutzung von Liegenschaften oder der Nachfrageförderung gewidmet haben, so stehen diese bislang wenig integriert nebeneinander. Und auch die von Industrie- und Handelskammern oder privaten Trägern angebotenen Existenzgründungs- und Professionalisierungsangebote, die explizit auf die besondere Situation der Kreativwirtschaft abgestimmt sind, gehören bislang eher zur Ausnahme.

\section{Steuerbarkeit der Kreativwirtschaft?}

Die Kreativwirtschaft zu lenken ist ein paradoxer Prozess (DeFillippi et al. 2007). Dabei gilt es zunächst zu verstehen, wie sich dieses Handlungsfeld entfaltet hat und wie das Verhältnis zwischen freiwilliger oder erzwungener (Selbst-)Steuerung als biographischer Entwurf zu verorten ist. Aufgrund ordnungspolitischer Gestaltungsansprüche sind Steuerungsprozesse immer mit Staat, seiner Fachadministration, seinen Instrumenten innerhalb kultureller Normen und Institutionen $\mathrm{zu}$ denken. Diese können immer direktional auf das Individuum eingreifen, z. B. im Kredit-, Finanz- und Steuerwesen. Es gilt daher zu überprüfen, inwiefern diese bereits etablierten Ausgleichs- und Kontrollinstrumente des Staates die z. B. unternehmerischen und künstlerischen Aktivitäten der Kultur- und Kreativproduzenten behindern oder unterstützen.

Die Kreativwirtschaft erfordert aber ein erweitertes Steuerungsverständnis, das hier als Kontextsteuerung angesprochen wird. Kontextspezifische Steuerungsperspektiven befragen das relationale Verhältnis zwischen z. B. urbanen Einbettungskontexten in Form von vielfältigen Arbeits-, Sozial- und Kommunikationsräumen. Ebenso steht die Frage des Zugangs zu Wissensnetzen und der Verfügbarkeit von wissensbasierten Innovationsquellen im Vordergrund, die von Akteuren und Netzwerken verwendet werden können. Ebenso verweist der jeweilige Kontext auf 
das spezifische Image, die Imagewirkung eines Standorts und die Imageerträge für die Profilierung dieser jungen Ökonomie.

Die Figur des Kompetenzträgers Akteur als Subjekt der Kreativwirtschaft wird abgelöst durch neue Zuschreibungen an die Berufe und Tätigkeiten sowie Selbstverständnisse der betreffenden Marktteilnehmer. An die Stelle des rational handelnden, allwissenden „Akteurs“, der autonom handelt, treten neue Formen und Verständnisse, die in der Figur des „unvollständigen Akteurs“ kulminieren (Priddat 2005). Neue Identitätszuschreibungen an Personen, Netzwerke und temporäre Kollektive gewinnen an Bedeutung. Es gilt daher Fragen an diese neuen Formen des institution building zu richten, und zu prüfen, welche Chancen mit ihnen einhergehen, um die Kreativwirtschaft zu fördern.

\section{Aufbau und Zielstellung der Anthologie}

Ist die Kreativwirtschaft steuerbar und wenn ja, wie und durch wen? Diese wesentliche Ausgangsfrage wird im vorliegenden Buch umfassend beantwortet. Um dieses Ziel zu erreichen, werden im Folgenden verschiedene Autoren ihr Wissen und ihre Sicht im Bezug auf den komplexen Themenbereich vermitteln. Die Struktur der Anthologie ist in insgesamt drei Teile gegliedert.

Der erste Teil gibt einen Überblick über verschiedene Dimensionen der Kreativwirtschaft. Klaus R. Kunzmann eröffnet die Beiträge und gibt einen Überblick über die Anwendung des Konzepts Kreativwirtschaft. Bastian Lange wendet den Begriff Governance auf die Kreativwirtschaft an und schlägt erste Ansatzpunkte vor, welche Governancedimensionen sich daraus ableiten lassen. Alain Thierstein, Agnes Förster und Stefan Lüthi erörtern die Steuerbarkeit von Kreativwirtschaft in und durch Metropolregionen. Alexa Färber befragt die Dimension Risiko, wie sie in der Kreativwirtschaft verhandelt wird. Sebastian Olma führt eine internationale Bestandsaufnahme der Kritik durch, die der Kreativwirtschaft und ihrer politischen Instrumentalisierung entgegengebracht wird, und spart dabei selber nicht an Kritik.

Der zweite Teil der Anthologie greift sechs zentrale Dimensionen der Kreativwirtschaft heraus und spiegelt konzeptionelle Beiträge mit Praxisbeispielen. Der erste Themenkomplex zur Arbeit wird durch Angela McRobbie eröffnet, die am Beispiel Londons zeigt, wie Marktteilnehmerinnen und Marktteilnehmer Arbeit in der Kreativwirtschaft bewältigen und organisieren. Hubert Eichmann und Stefan Leitner-Sidl stellen die Arbeitsbedingungen der Wiener Kreativwirtschaft am Beispiel des Projekts Rochuspark vor. Bastian Lange und Inga Wellmann 
zeigen die soziale Verräumlichung von Arbeitsbeziehungen sowie die spontane Nutzung von Raum anhand so genannter Co-working Spaces auf.

Der zweite Themenkomplex zu Kompetenzen wird durch einen theoretischen Beitrag von Harald A. Mieg eingeleitet. Sebastian Dresel erklärt am Beispiel der Pop-Akademie das Mannheimer Modell. Zukunftsweisende Entwicklungsstrategien für postindustrielle Standorte stellt Judith Seng anhand des Projekts Design Reaktor an der Universität der Künste Berlin vor.

Der dritte Teil skizziert die Emergenz neuer Organisationsformen und Institutionen in der Kreativwirtschaft. Inga Wellmann beschreibt Hybridisierungsphänomene in Akteurs- und Organisationsstrukturen als Zeichen einer für die Netzwerkgesellschaft bezeichnenden Schnittstellenkultur. Als Fallbeispiel verdeutlicht ein Interview mit dem Hybrid Space Lab, welche integrativen und transdisziplinären (Raum-)Strategien erforderlich sind, um einer auf allen Ebenen des Gestaltbaren wachsenden Komplexität zu begegnen.

Der vierte Teil widmet sich dem Thema Urban Imagineering, also der Frage, wie Städte inszeniert werden - verbal, aber auch visuell, wie zum Beispiel durch Architektur und Städtebau. Chris Steyaert und Timon Beyes diskutieren verschiedene politische Narrative der unternehmerischen Stadt und plädieren für ein differenziertes Nachdenken über Stadt und Kultur. Birgit Stöber und Can Seng Ooi illustrieren am Beispiel des Stadtstaates Singapur, wie Kultur sehr weit gedacht und instrumentalisiert wird, um urbane Bilder und Wahrnehmungen zu beeinflussen, und zwar in einem international verzweigten GovernanceNetzwerk.

Der fünfte Teil wendet sich dem Bereich Marketing und Urban Branding zu. Birgit Stöber und Ares Kalandides differenzieren beide Begriffe und fragen, inwiefern Städte als Hauptzentren der Kreativwirtschaft überhaupt als Brands vermarktet werden können. Björn Böhning stellt am Beispiel der Stadt Berlin vor wie strategisch daran gearbeitet wird kreative Potenziale zu vermarkten.

Der sechste Teil führt das Themenfeld wiederum stärker an den Begriff Governance heran. Hans-Joachim Bürkner beschreibt die Herausforderungen für lokale Politik und Governance, die von der Entwicklung städtischer Nischenökonomien ausgehen. Constanze Günther und Achim Prossek skizzieren anhand der Internationalen Bauausstellungen IBA Emscher Park und IBA Hamburg, wie sich die Profilbildung durch Projekte vom Fokus Kultur hin zu Kreativität gewandelt hat.

Der dritte und letzte Teil der Anthologie stellt Zukunftsperspektiven dar. Andy Pratt überdenkt das Verhältnis von Kultur und Wirtschaft 
sowie die Kulturpolitik der vergangenen fünfzig Jahre im internationalen Kontext. Dabei diskutiert Pratt die „Kulturalisierung“ der Wirtschaft sowie die Instrumentalisierung von Kultur und plädiert für eine neue Kulturpolitik. Pernille Askerud setzt den Diskurs über Kreativität in einen internationalen Kontext von wachsenden Ungleichheiten und fragt nach dem gesellschaftlichen Transformationspotenzial der Kreativwirtschaft. Bernhard Krusche erörtert, inwiefern sich Städte in Zukunft stärker als bislang mit dem Umstand auseinandersetzen müssen, in ihrer Struktur, Funktion und Entwicklung auf Berechenbarkeit und hierarchische Ordnungsprinzipien zu verzichten. Was heißt dies für die zukünftige Stadt und ihre Steuerbarkeit?

Die Herausgeber dieser Anthologie greifen abschließend die Eingangsfrage auf und geben Antworten. Anhand von zehn Punkten werden Governanceperspektiven der Kreativwirtschaft eröffnet.

Die Herausgeber bedanken sich ganz herzlich beim transcript Verlag für die Möglichkeit ein junges Forschungsfeld einer größeren Öffentlichkeit vorstellen zu können. Besonderer Dank gebührt den Autorinnen und Autoren, die sich mit ihren Beiträgen auf die komplexen Fragen und Situationen der Kreativwirtschaft eingelassen und sich ihren unterschiedlichen Bewertungen nicht verwehrt haben.

Großen Dank schulden die Herausgeber Harald A. Mieg für die kontinuierliche Unterstützung und nachhaltigen Ermutigungen im Rahmen des Forschungskreises Governance der Creative Industries am GeorgSimmel-Zentrum für Metropolforschung an der Humboldt Universität zu Berlin.

Die Herausgeber möchten sich außerdem ganz herzlich bei Corinna Kennel für wichtige Unterstützung zum Umgang mit dem Begriff und Konzept Governance bedanken. Weiterer Dank gilt Katja Manz für geduldiges Layouten und der Arbeit an der Druckvorstufe sowie Anna Stüler von trojan books für das Lektorat.

\section{Literatur}

Althans, Birgit/Audehm, Kathrin/Binder, Beate, et al. (2008): „Kreativität. Eine Rückrufaktion“. In: Zeitschrift für Kulturwissenschaften 1, S. 7-13.

BMWi (Hg.) (2009): Gesamtwirtschaftliche Perspektiven der Kulturund Kreativwirtschaft in Deutschland. Berlin: BMWi, Forschungsbericht Nr. 577, $35 \mathrm{~S}$. 
Brand, Ulrich (2004): „Governance“. In: Ulrich Bröckling/Susanne Krasmann/Thomas Lemke (Hg.): Glossar der Gegenwart, Frankfurt/Main: Suhrkamp, S. 111-117.

Cars, Göran/Healey, Patsy/Madanipour, Ali, et al. (Hg.): (2002) Urban Governance, Institutional Capacity and Social Milieux, Aldershot: Ashgate.

DeFillippi, Robert/Grabher, Gernot/Jones, Candace (2007): „Introduction to paradoxes of creativity: managerial and organizational challenges in the cultural economy“. In: Journal of Organizational Behavior 28 (5), S. 511-521.

Friebe, Holm/Lobo, Sascha (2006): Wir nennen es Arbeit oder: Intelligentes Leben jenseits der Festanstellung, München: Heyne.

Friebe, Holm/Ramge, Thomas (2008): Marke Eigenbau. Der Aufstand der Massen gegen die Massenproduktion, Frankfurt/Main: Campus Verlag.

Grabher, Gernot (2004): Temporary Architectures of Learning: Knowledge Governance in Project Ecologies. Organization Studies 25 (9), S. 1491-1514.

Grabher, Gernot/Ibert, Oliver (2004): Produktion in Projekten. Das Beispiel der Werbebranche in Hamburg und der Softwareproduktion in München (Abschlussbericht des DFG Projektes). Bonn: Geographisches Institut Universität Bonn, $21 \mathrm{~S}$.

Gruber, Petra C. (Hg.) (2008): Nachhaltige Entwicklung und Global Governance: Verantwortung. Macht. Politik, Opladen: Budrich.

Haus, Michael/Heinelt, Hubert/Stewart, Murray (Hg.) (2005): Metropolitan Governance: Capacity, Democracy and the Dynamics of Place, London: Routledge.

Healey, Patsy (2004): „Creativity and Urban Governance“. In: Policy Studies 25 (2), S. 87-102.

Kooiman, Jan (2003): Governing as Governance, London: Sage.

Kötschau, Kerstin (2008): Good Governance and Developing Countries: Interdisciplinary Perspectives, Frankfurt/Main [u. a.]: Lang.

Lange, Bastian (2007): Die Räume der Kreativszenen. Culturepreneurs und ihre Orte in Berlin, Bielefeld: transcript Verlag.

Lange, Bastian (2008): „Accessing Markets in Creative Industries - Professionalization and Social-Spatial Strategies of Culturepreneurs in Berlin“. Creative Industries Journal 1 (2), S. 115-135.

Lange, Bastian/Kalandides, Ares (2008a): „Governance of Creative Industries in Berlin - Paradoxes, Places, Practices“. In: Wan, Helen/ Yueng, Erin/Yueng, Theresa (Hg.): When Creative Industries Crossover with Cities, Hongkong: Hong Kong Institute of Planners (HKIP), S. 122-133. 
Lange, Bastian/Kalandides, Ares/Stöber, Birgit, et al. (2008b): „Berlin’s Creative Industries: Governing Creativity?“ In: Industry and Innovation 15 (5), S. 531-548.

Lorey, Isabell (2007): „Vom immanenten Widerspruch zur hegemonialen Funktion. Biopolitische Gouvernementalität und Selbstprekarisierung von Kulturproduzenten“. In: Gerald Rauning/Ulf Wuggenig (Hg.): Kritik der Kreativität, Wien: Turia + Kant, S. 121-136.

Manske, Alexandra (2008): „Kreative Superstars. Die soziale Platzierungsstrategie der Digitalen Bohème“. In: Polar. Zeitschrift für Politik. Theorie. Alltag, Märzausgabe, Suhrkamp, S. 123-126.

Matthiesen, Ulf/Bürkner, Hans-Joachim (2004): „Wissensmilieus - Zur sozialen Konstruktion und analytischen Rekonstruktion eines neuen Sozialraum-Typus“. In: Ulf Matthiesen (Hg.): Stadtregion und Wissen - Analysen und Plädoyers für eine wissensbasierte Stadtentwicklung, Wiesbaden: Verlag für Sozialwissenschaften, S. 65-89.

Priddat, Birger P. (2005): Unvollständige Akteure: komplexer werdende Ökonomie, Wiesbaden: Verlag für Sozialwissenschaften.

Sick, Sebastian (2008): Corporate Governance in Deutschland und Großbritannien: ein Kodex- und Systemvergleich, Baden-Baden: Nomos.

Wenger, Etienne (1999): Communities of Practice, Cambridge: Cambridge University Press. 



\section{Kreativwirtschaft und strategische Stadtentwicklung}

KLAUS R. KUNZMANN

Die Erwartungen sind hoch. Städte in allen Regionen der Welt haben sich in den letzten Jahren auf ein Handlungsfeld gestürzt, mit dessen Hilfe sie hoffen, die strukturellen Herausforderungen der Globalisierung meistern zu können. Ermutigt insbesondere durch Veröffentlichungen von Richard Florida und Charles Landry, setzen sie zu Beginn des Beginn des 21. Jahrhunderts mit der Kreativ- und Kulturwirtschaft auf zwei bislang wenig beachtete kommunale und regionale Handlungsfelder. Sie galten lange Zeit als unergiebig, weil sie in den Aktionskatalogen der Wirtschaftsförderer und den Lehrbüchern der Business Schools nicht vorkamen. Zudem waren die städtischen Akteure ohnehin der Meinung, dass Kultur vom öffentlichen Sektor immer subventioniert, oder vom privaten Sektor grundsätzlich gesponsert werden muss. Das Umdenken hat inzwischen eingesetzt, nicht zuletzt dank der von den Medien so gerne verbreiteten Auffassung, dass es die post-industrielle „kreative Klasse“ ist, die Städte heute attraktiv und lebenswert, also wettbewerbsfähig macht, und dass eine Stadt, die erfolgreich sein will, ohne „Talente" nicht auskommt.

\section{Kreativwirtschaft: noch immer ein "fuzzy“ Konzept}

Die Tatsache, dass „Kreativität“ in allen Schichten der Bevölkerung ausschließlich positive Assoziationen weckt, hat sehr wesentlich zum Siegeszug der Kreativwirtschaft beigetragen, jedenfalls zu ihrem Siegeszug in den Medien und in der Stadtpolitik. Aber auch die schnelle Umetiket- 
tierung von ,innovativ“ zu „kreativ“ hat geholfen. Was vor einem Jahrzehnt noch innovativ war, ist heute ganz einfach kreativ.

Dies weist darauf hin, dass trotz aller Bemühungen um eine einheitliche Definition in Europa, in Deutschland und anderswo, Kreativwirtschaft noch immer sehr unterschiedlich kategorisiert und abgegrenzt werden kann. Gehört der kreative Banker dazu, der Milliarden spekulativ transferiert und dabei diese Milliarden aus den Augen verliert, oder doch eher der Geigenbauer, der sein handwerkliches Können für ein Produkt nutzt, ohne das kein Orchester der Welt auskommt. Ist aber jeder Musiker per se kreativ, ein Automechaniker, der Oldtimer zum Fahren bringt, aber nicht, oder ist es die Steuerfachfrau, die kunsthandwerkliche Produkte in Nebentätigkeit herstellt. Graffiti Künstler sind sicher kreativ, aber die Statistik erhebt sie nicht, auch nicht kreative Menschen in der Landwirtschaft. Kreative Menschen arbeiten immer an Rändern, an Grenzen. Auch kreative Räume sind immer Grenzräume für Neuentdeckungen.

Alle Versuche, Kreativwirtschaft umfassend und solide zu definieren, müssen scheitern, weil die Ränder zwangsläufig immer unscharf bleiben. Aber für eine zukunftsorientierte und nachhaltige Stadtpolitik und auch sie erfordert kreative Menschen, die sie formulieren und umsetzen - ist es letzten Endes auch nicht entscheidend, ob ein lokaler Videokünstler Kunst erzeugt oder einfach nur Geschäfte macht. Und dies gilt für alle Handlungsfelder, auf denen Stadtpolitik Kreativwirtschaft fördern soll und kann.

Also, Definitionen und Abgrenzungen spielen keine so große Rolle, wenn es darum geht, Kreativwirtschaft an einem Ort zu fördern. Nur zwei Dinge sind wichtig: Erstens bedarf es guter Kenntnis der endogenen Potenziale der Kreativwirtschaft, weil sie der Ausgangspunkt für Fördermaßnahmen sind. Zweitens muss in einem Handlungsraum Konsens unter den Beteiligten gefunden werden, was sie grundsätzlich unter Kreativwirtschaft verstehen und was sie gegebenenfalls fördern wollen. Dabei sollten selbstverständlich diejenigen nicht ausgeschlossen werden, die sich nachvollziehbar als Kreative bezeichnen, aber nicht der vorgegeben Definition entsprechen. Es macht grundsätzlich ja auch wenig Sinn, kreative Räume als Nutzungskategorie in einem kommunalen Flächennutzungsplan auszuweisen, denn dies führt unweigerlich dazu, dass solche Räume lediglich vom Immobilienmarkt als kreative Räume vermarktet werden, während die wirklich Kreativen ganz anderswo tätig sind. Noch etwas ist wichtig: Die Kreativwirtschaft lebt und floriert mit der Intensität der Kommunikation zwischen ihren Akteuren. Wirtschaftsförderer, die jahrelang Unternehmen der Logistik gefördert haben, werden sich schwer tun, die Zielgruppe der wirklich Kreativen zu 
verstehen und glaubhaft anzusprechen. Nicht selten werden aus Begeisterung für die neu entdeckte Zielgruppe Bauten für die Kreativwirtschaft erstellt, die diese dann gar nicht nutzen kann, weil sie die hohen Kosten für Erwerb oder Miete nicht aufbringen kann, denn es wird auch oft vergessen, dass Kreativität und Prekariat sehr oft zusammenhängen.

War es vor Jahren noch das Unwissen über die Kreativwirtschaft, das dazu führte, dass Wirtschaftsförderer und Politikberater kein Interesse hatten, sich mit der Kreativwirtschaft zu befassen, so hat das Thema heute als Handlungsfeld weltweit einen festen Platz in der Stadtpolitik. Mit dem Hinweis auf die Bedeutung von kreativen Unternehmen und talentierten Bürgern für die Zukunft der Stadt, bemüht sich jede große Stadtverwaltung heute, qualifizierte, kreative, innovative Unternehmen und Arbeitskräfte anzulocken. Keine Stadt, die weltweit Aufmerksamkeit sucht, kann es sich heute leisten, die Kreativwirtschaft zu vernachlässigen, wie es noch vor einem Jahrzehnt der Fall war.

Auch wenn jede Stadt bemüht ist, ihr eigenes Profil zu finden und zu vermarkten, die Kreativwirtschaft gehört zu Beginn des 21. Jahrhunderts zu diesem Profil und damit selbstverständlich zu den Segmenten der Wirtschaft, die die Zukunftsfähigkeit der Städte garantieren sollen. $\mathrm{Ob}$ die große Finanzkrise 2008/2009 daran etwas ändert, darüber kann nur spekuliert werden. Sie kann die neue Begeisterung für die Kreativwirtschaft noch befördern, weil die öffentlichen Mittel in die Sanierung von Banken und großen Technologieunternehmen fließen, und Kreative, eben ohne staatliche Unterstützung, kreativ überleben müssen. Sie kann aber auch dazu führen, dass sich Meinungen wieder durchsetzen, die die kulturell geprägte Kreativwirtschaft als ein Luxushandlungsfeld für Zeiten des Wohlstands verstehen.

\section{Kreativwirtschaft und kreative Städte zwischen Los Angeles, Melbourne und Berlin}

In vielen Städten Europas, Nordamerikas, Ostasiens und Australiens ist die Kreativwirtschaft in den letzten beiden Jahrzehnten zu einem besonderen Faktor der Stadtentwicklung geworden. Jede Stadt, die auf den mehr oder weniger seriösen Rankinglisten der Wirtschaftsjournale einen Platz in der oberen Liga einnehmen möchte, beauftragt ihre Marketingagenturen, sie auch als kreative Stadt zu vermarkten.

Los Angeles gilt manchen als Wiege der Kreativwirtschaft, zum einen weil dort die Filmindustrie traditionell weltweit ausstrahlt, aber auch, weil darüber in den beiden letzten Jahrzehnten mehr veröffentlich wurde, als über die dort ebenfalls ansässige Rüstungsindustrie, die letzt- 
lich auch viele Ingenieure beschäftigt, welche - wenn es darum geht, militärische Produkte zu verbessern - auch sehr kreativ sein müssen. Natürlich hat Kreativität in New York, in welchen Formen auch immer, eine lange Tradition. Aber auch Städte wie Toronto oder Minneapolis sind Orte mit einem hohen Anteil an kreativen Arbeitsplätzen und meist innerstädtischen Quartieren, in denen diese Arbeitsplätze entstehen. Toronto bediente sich der Aufmerksamkeit, die Richard Florida inzwischen überall in der Welt genießt, in dem es ihn zum Direktor eines großzügig ausgestatteten Forschungszentrums machte. Minneapolis ist es in den letzten Jahren gut gelungen, das kulturelle und kreative Image der Stadt aufzuwerten und zu sichern. Pittsburgh hatte schon vor über einem Jahrzehnt gezielt und mit einigem Erfolg Kulturwirtschaft in die daniederliegende Innenstadt der altindustriellen Stadtregion gelockt, um das negative Image der Stadt aufzuwerten und die Innenstadt als sicheren Aufenthaltsort attraktiv zu machen. Aber auch an den zersiedelten, gesichtslosen Rändern amerikanischer Stadtregionen entstehen an Orten, an denen der Immobilienmarkt nicht mehr besonders interessiert ist und aktive Gruppen der zivilen Gesellschaft sich engagieren, gelegentlich kreative Quartiere, die zwar letztlich nur lokale Bedeutung haben, aber damit auf regionale strukturelle und gesellschaftliche Veränderungen reagieren.

In Australien wetteifern Melbourne und Sydney um die Palme der kreativen Stadt, in der die Kreativwirtschaft blüht. Da der gesamte Kontinent unter seiner Geschichte und der großen Entfernung zu Europa und Ostasien leidet, liegt es der Stadtpolitik und ihren Beratern in den beiden Städten sehr am Herzen, Kultur und Kreativität zu fördern. Zielgruppen der Politik sind in erster Linie die Bewohner der Städte, doch es geht auch darum, australische Städte für kreative Menschen aus Ostasien attraktiv zu machen. Hier werden dann Hochschulpolitik, Wirtschaftspolitik und Kulturpolitik eng miteinander verknüpft. Die Zahl der chinesischen Studierenden, die statt nach Nordamerika und Europa nach Australien kommen, ist jedenfalls in den letzten Jahren sehr angestiegen.

Beijing, lange Zeit „nur“ politisches Zentrum Chinas, hat sich in den letzten Jahren auch zu einem Zentrum der chinesischen Kulturwirtschaft entwickelt, das auch international Aufmerksamkeit gefunden hat. Die Stadt sieht sich als die Stadt der Kultur, Kreativität und der Kreativwirtschaft. Der kulturwirtschaftliche Leuchtturm der Stadt ist das Quartier 798, das inzwischen weltweit als Produktions- und Ausstellungsort moderner chinesischer Kunst bekannt ist. Diese noch zu Zeiten der ehemaligen DDR errichtete Produktionsstädte für militärisch genutzte elektronische Güter hat in der Folge schnell weitere Initiatoren dazu inspiriert, ähnliche Quartiere an anderen, nicht so bekannten Orten in der Stadt zu 
entwickeln. Dort haben sich dann die (wirklich) Kreativen niedergelassen, die sich in dem inzwischen zur Touristenattraktion gewordenen Kulturwirtschaftsquartier nicht mehr wohl fühlen. Ebenso wie Hongkong hat auch Taipei auf Taiwan schon vor Jahren die Symbolkraft und Medienwirksamkeit der kreativen Stadt „entdeckt“. Seitdem bemüht sie sich die Stadt über die Förderung von Kultur- und Kreativwirtschaft ihr internationales Profil zu schärfen. Doch die international wirkenden lokalen Potenziale der Kreativwirtschaft - letztlich vor allem die in Taiwan sehr weit entwickelte Elektronikindustrie - sind nicht sehr groß.

Kyoto, aber auch Osaka sind zwei Städte in Japan, die sich aufgrund ihrer traditionellen endogenen Potenziale - in beiden Städten gibt es eine lange Tradition der Produktion von kulturbezogenen Gütern - mit einigem Erfolg bemühen, die Chancen der Kreativwirtschaft zu nutzen. Auch in Tokyo gibt es Segmente der Kreativwirtschaft, doch ihre Bedeutung ist dort gegenüber anderen Branchen nicht überdurchschnittlich groß. Ein Grund dafür sind sicher die extrem hohen Grundstücks- und Wohnungspreise und Lebenshaltungskosten, die es dem kreativen Prekariat dort fast unmöglich machen ihre Kreativität zu entfalten.

Was für die USA und Asien gilt, das gilt auch für Europa. Ob London oder Paris, ob Amsterdam, Antwerpen, Wien oder Mailand, Zürich, Kopenhagen oder Helsinki, alle Städte vermarkten sich als europäische Metropolen, in denen die Kreativwirtschaft eine besondere Bedeutung und internationale Ausstrahlung hat.

Nicht ohne guten Grund hat sich Berlin in den letzten Jahren zur heimlichen Hauptstadt der Kreativwirtschaft in Deutschland hochstilisiert. Vielleicht liegt es daran, dass die Stadt, die noch vor hundert Jahren das globale Zentrum der Elektrowirtschaft war, keine anderen wirtschaftlichen Schwerpunkte aufweisen kann. Vielleicht auch daran, dass die Stadt, die ein hohes Kulturbudget vorweisen kann, wie keine andere Stadt im Westen Europas, preiswerte innenstadtnahe Wohn- und Arbeitstätten hat.

Aber auch ganze Regionen in Europa möchten kreativ sein. Flandern in Belgien und Venetien in Italien sind zwei Regionen, in denen eine Vielzahl von kleinen und mittleren Unternehmen der Kreativwirtschaft angesiedelt sind, und wo institutionalisierte Bemühungen die vorhandenen Cluster der Kreativwirtschaft vernetzen und stärken, so dass sie sich auf diese Weise international profilieren

Schließlich sieht inzwischen jede Kulturhauptstadt Europas in einem medienwirksamen Ereignis eine Chance, die lokale Kreativ- und Kulturwirtschaft zu fördern. Dies hat insbesondere Dortmund veranlasst, sich im Rahmen des Kulturhauptstadtjahres 2010 als neues Zentrum für Kulturwirtschaft zu ,positionieren“. Aus den Beobachtungen von Städ- 
ten, die sich als kreative Städte profilieren und sich als kreativwirtschaftliche Zentren vermarkten, lassen sich unabhängig von den jeweiligen kulturellen und wirtschaftlichen Bedingungen folgende Hinweise zur Förderung der Kulturwirtschaft ableiten. Sie definieren das soziale, kulturelle wirtschaftliche und politische Umfeld der Kreativwirtschaft in einer Stadt, das die Produktionsbedingungen der Kreativwirtschaft charakterisiert und bestimmt:

Kreativwirtschaft und Kulturleben: Der Zusammenhang zwischen Kreativwirtschaft und städtischen Kulturleben bzw. Kulturangeboten ist offensichtlich. Kulturwirtschaft fördern heißt daher immer auch das kommunale Kulturleben mit hoher politischer Priorität fördern. Städte, die sich als Städte der Kulturwirtschaft profilieren möchten, werden dies ohne Erhöhung ihrer Kulturetats nicht glaubhaft und effizient tun können, es sei denn sie haben großzügige Mäzene am Ort, die zusätzlich einspringen um kulturelle Infrastruktur zu bauen und zu unterhalten sowie kulturelle Ereignisse finanziell zu unterstützen.

Kreativwirtschaft und Events: Nur immer wiederkehrende kulturelle Events festigen das kulturwirtschaftliche Profil eine Stadt. Nur sie schaffen die Bedingungen vor Ort, die eine Kulturwirtschaft braucht, um nachhaltig lokale und internationale Abnehmer für Produkte und Dienstleistungen $\mathrm{zu}$ finden. Die Einrichtung international ausstrahlender Events ist nicht leicht. Sie bedarf vor allem profilierter Persönlichkeiten, die solche Events repräsentieren. Es gilt, diese Persönlichkeiten an den Standort zu holen und dort physisch und emotionell zu binden.

Kreativwirtschaft und Kulturhauptstadt: Der Titel Kulturhauptstadt macht aus einer Stadt nicht sofort auch eine kreative Stadt mit einer blühenden Kreativwirtschaft. Er ist eine Chance für die Kulturwirtschaft am Ort, soweit das Ereignis genutzt wird, um die lokalen Rahmenbedingungen der Kulturwirtschaft auch über das Festivaljahr hinaus zu verbessern. Eine einjährige Party allein sichert keine Arbeitsplätze am Ort, auch wenn mediale Ausstrahlungseffekte der Veranstaltung grundsätzlich dazu beitragen, das Bewusstsein für einen höheren Stellenwert der Kulturwirtschaft in lokalen politischen und wirtschaftlichen Milieus zusätzlich zu verbessern. Internationale Medien vergessen schnell, dass eine Stadt Kulturhauptstadt war.

Kreativwirtschaft, Geschichte und Identität: Die Kreativwirtschaft, insbesondere die Kulturwirtschaft, hat an einem Ort in der Regel eine lange Geschichte. Sie ist wesentliches Element lokaler Identität. Unternehmen und Kreative nutzen das Wissen, das an diesen Ort über Jahrzehnte angesammelt wurde und das diesen Ort mitgestaltet hat. Dieses Wissen manifestiert sich in einer Stadt sichtbar in Form von Bauten, Straßenzügen, öffentlichen Plätzen, Denkmälern oder besonderen Quar- 
tieren. Diese Geschichte ist Teil des endogenen Kapitals der Kulturwirtschaft an einem Ort. Neue kulturwirtschaftliche Traditionen zu schaffen, erfordert besondere Initiativen, „windows of opportunities“ aber auch Persönlichkeiten, die sich damit identifizieren, und nicht zuletzt auch große finanzielle Anstrengungen.

Kreativwirtschaft und Internationalität: Kreativwirtschaft hat starke lokale Wurzeln. Doch in Zeiten fast grenzenloser Internationalisierung sind die Akteure der Kulturwirtschaft gezwungen, ebenso wie andere Industriezweige auch, sich dauerhaft international zu vernetzen. Bei aller Ortsgebundenheit können sie nicht nur auf lokale und regionale Märkte vertrauen. Dies hat vielfältige Implikationen für individuelle Mobilität sowie für den Zugang zu Informations- und Kommunikationsmedien, aber auch für nach außen orientierte Informations- und Marketingstrategien.

Kreativwirtschaft und gesellschaftliche Offenheit: Gerade in Deutschland ist Kreativwirtschaft noch immer eine sehr deutsche Angelegenheit, die in besonderem Maße auf den endogenen Traditionen im Lande aufbaut. Doch andere Kulturen, und damit andere Erfahrungen, Fähigkeiten, andersartige Gewohnheiten und divergierende Bedürfnisse, sind neue Chancen für die Entwicklung der Kulturwirtschaft, insbesondere in Deutschland. Dies wiederum macht es notwendig, Personen für innovative Initiativen auf diesem Gebiet zu fördern, an Ausbildungsstätten wie im lokalen Kulturleben, in der Wirtschaftsförderung wie in der Stadtpolitik.

Kreativwirtschaft und Stadtgestalt: Die in aller Welt zu beobachtenden Reurbanisierungsbewegungen und die Renaissance der Innenstädte stehen an vielen Orten im Zusammenhang mit dem Wachstum der Kreativwirtschaft. Erfahrungen zeigen, dass moderne Siedlungen an den Rändern der Städte keine guten und anregenden Milieus für die Kreativwirtschaft darstellen. Selbst Technologieparks bemühen sich zunehmend, und dies nicht ohne Schwierigkeiten, ihre offensichtlichen Urbanitätsdefizite abzubauen und Nachrüstungsstrategien zu entwickeln, deren Standortbedürfnisse in den Innenstädten besser befriedigt werden können, insbesondere dort wo Arbeitsstätten und Wohnorte zunehmend verschmelzen.

Noch etwas: Kreativwirtschaft überschneidet sich in vielen Bereichen mit Wissensindustrien, insbesondere mit den Unternehmen und Unternehmungen, die aus den Universitäten und Forschungszentren hervorkommen. Die Förderung von Wissenschaft und Forschung, und dies ist in der Regel nur am Rande eine Aufgabe von Städten, ist also auch gezielte Förderung der Kreativwirtschaft. 
Hier gibt es Synergien und Effekte der gegenseitigen Unterstützung. Auf einem kommunalen Politikfeld gibt es in dieser Hinsicht jedoch immer Handlungsbedarf. Die Wohn- und Freizeitbedürfnisse der Kreativen und der Wissensarbeiter sind in der Regel sehr ähnlich, insbesondere was innerstädtische Standorte und Ziele anbelangt. Daher muss Stadtentwicklungsplanung dies im Auge haben.

\section{Strategische Stadtentwicklung durch Kreativ- und Kulturwirtschaft}

Doch was kann realistischerweise getan werden, um die Kreativwirtschaft an einem Ort im Rahmen strategischer Stadtentwicklungsplanung, und damit verbundener Wirtschaftsförderung und kommunaler Kulturpolitik konkret zu fördern? Zehn Ansatzpunkte für Handlungsfelder seien hier angesprochen, in denen die Kulturwirtschaft, also der besondere kreative Kern der Kreativwirtschaft, gefördert werden kann. ${ }^{1}$

Informationsgrundlagen schaffen und kulturwirtschaftliche Potenziale erkunden: Städte und Regionen kennen ihre kulturwirtschaftlichen Potenziale kaum oder jedenfalls nur bruchstückhaft. Die amtliche Statistik spiegelt in der Regel die Arbeitsplätze und Umsätze in den verschiedenen Bereichen der Kreativ- und Kulturwirtschaft nur unzureichend wider. Sonderauswertungen der kulturwirtschaftlichen Potenziale liegen selten vor. Hinzu kommen die unvermeidbaren Definitions- und Abgrenzungsprobleme. Auch bei Industrie- und Handelskammern, wie bei Handwerkskammern, liegen zumeist keine Daten vor. Ähnlich sieht es bei Wirtschaftsförderungsagenturen und Kulturämtern aus, zum einen weil sie die Kulturwirtschaft nicht für so wichtig erachten oder weil sie sich nicht legitimiert fühlen, sich damit zu befassen. Doch solide und glaubhafte quantitative Informationen über die regionalen Dimensionen und Potenziale der Kreativ- und Kulturwirtschaft sind unverzichtbar, wenn es darum geht, Cluster zu etablieren und Allianzen für die Förderung der Branche in einer Region zu schmieden.

Kulturwirtschaftliche Raumbeobachtung etablieren und Wettbewerber beobachten: Sind die regionalen Informationsgrundlagen gelegt und erste kulturwirtschaftsbezogene Initiativen auf den Weg gebracht, gilt es, die wirtschaftliche, kulturelle und städtebauliche Entwicklung konti-

1 Diese zehn Punkte beruhen in wesentlichen Teilen auf einem Beitrag des Autors für die Zeitschrift Aus Politik und Zeitgeschichte (siehe APuZ 3435/2006, S. 3-7). 
nuierlich weiterzuverfolgen. Der Verweis auf die festgestellte Dynamik des Sektors macht es leichter, weitere Verbündete zu überzeugen, laufende Initiativen zu unterstützen, auszubauen, zu korrigieren und zu ergänzen - und neue Projekte und Initiativen zu starten. Die Wettbewerber in Europa sollten dabei immer im Auge behalten werden, um von anderen Regionen im europäischen Raum zu lernen, wie diese Kreativ- und Kulturwirtschaft im Rahmen strategischer Stadtentwicklung nutzen und fördern. Dabei sind gerade solche - meist altindustrielle Regionen - von Interesse, die überregional nicht als „Kulturregionen“ bekannt sind, wie Sheffield Manchester oder Pittsburgh oder auch Turin, Zürich, Lille, Antwerpen oder Lyon.

Botschaften aussenden und Netzwerke bilden: Die Erfahrung hat gezeigt, dass die Kommunikation zwischen Akteuren der Kreativ- und Kulturwirtschaft und solchen des Kulturlebens immer noch von erheblichen Missverständnissen geprägt ist. Nach wie vor gilt es Skeptiker des „reinen“ Kulturlebens zu überzeugen, dass die Kulturwirtschaft der Kultur nicht schadet. Ebenso gilt es, die Wirtschaftsverbände immer wieder davon zu überzeugen, dass die Kulturwirtschaft eine für die lokalen Ökonomien wesentliche Branche ist, und nicht etwas, was gesponsert werden soll oder was staatlicher Förderung überlassen werden kann. So können regionale und lokale Strategien entwickelt werden, die bestehende Arbeitsplätze sichern oder neue schaffen, denn weder bei der regionalen Tourismusförderung noch bei der zukunftsorientierten Innenstadtpolitik kann auf die Kreativ- und Kulturwirtschaft als Branche verzichtet werden. Da insbesondere die Akteure der engeren Kulturwirtschaft aus vielerlei Gründen bis heute ihre Interessen kaum gemeinsam vertreten, fehlt auch die Lobby, welche die Interessen der Unternehmen in der Öffentlichkeit glaubhaft und machtvoll vertreten könnte, wie es etwa die Straßenbau-Lobby so selbstverständlich tut. Notwendig ist daher der Aufbau von nachhaltigen Interessennetzwerken vor Ort bzw. in der Region. Solche Netzwerke von Personen entstehen, wenn es Gelegenheiten zur Kommunikation gibt und wenn sich einzelne, in den jeweiligen Milieus bekannte und anerkannte Persönlichkeiten dafür auch stark machen.

Erfolgsgeschichten verbreiten: Marketingfachleute wissen, dass nichts erfolgsfördernder ist als der Erfolg; sie wissen um die mediale Wirkung von Erfolgsgeschichten. In der politischen Arena ist es immer leichter, auf realisierte Projekte hinzuweisen, als neue Prozesse mit unsicherem Ausgang in Gang zu setzen. Erfolgsgeschichten aus anderen Städten regen an, auch wenn sie meist nicht kopiert werden können, weil die Bedingungen vor Ort unterschiedlich sind oder Schlüsselakteure fehlen. Dennoch, eine breite Kommunikation erfolgreicher Projekte zur 
Förderung der regionalen Kreativ- und Kulturwirtschaft ist für eine bessere Akzeptanz unverzichtbar. Die regionalen Medien sowie die vielen meinungsbildenden Organe der Wirtschaftsverbände und Wochenjournale haben dabei eine Schlüsselrolle inne. Wichtig ist deshalb die direkte Ansprache der Journalistinnen und Journalisten.

Katalytische Projekte initiieren: Erfolgsgeschichten sind wichtig, doch geraten sie schnell in Vergessenheit. Nachhaltiger sind die Erfahrungen, die alle am Gelingen des Projektes beteiligten Personen gemacht haben und mit denen sich innovative kulturwirtschaftliche Projekte auf den Weg bringen lassen. Sie haben eine besondere katalytische Funktion, weil sie Akteure aus ganz unterschiedlichen Politik- und Handlungsfeldern zusammenbringen und diese dann in einem vorgegebenen Zeitrahmen, und mit festen politisch vorgegebenen Terminen kooperieren müssen. Der Erfolgs- und Zeitdruck ist es, der dazu beiträgt, dass Entscheidungen schneller getroffen und Bedenken zurückgestellt werden, dass Koalitionen eingegangen sowie Feindbilder und Vorurteile abgebaut und neue Finanzierungsmöglichkeiten gefunden werden. Projekte im Handlungsfeld Kulturwirtschaft haben diesen katalytischen Charakter. Sie sind - weit mehr als andere Routineprojekte der Stadtentwicklung und Wirtschaftsförderung - auf motivierte und sachlich interessierte Personen angewiesen und daher Experimentierfelder für eine zukunftsorientierte stadtregionale Wirtschaftsförderung, weit über die Kulturwirtschaft hinaus.

Kreative Räume für Experimente und Innovationen offen halten: Stadträume, deren Nutzungen bis zum letzten Quadratmeter definiert und gesetzlich festgelegt sind, lassen wenig Spielräume für Neues, schon gar nicht für Experimente, die auch fehlschlagen können. Jede Stadt braucht Räume, deren Nutzung durch kreative Menschen neu definiert werden können. Solche Räume gibt es in jeder Stadt. Wenn politisch gewollt, lassen sich diese Art Umnutzungsprozesse in benachteiligten, und vom Immobilienmarkt vernachlässigten Quartieren durch die Lokalisierung von kulturbezogenen Nutzungen und Ausbildungsstätten, durch Ankerprojekte und sozio-kulturelle Zentren an gewünschten Standorten initiieren bzw. beschleunigen. Dies gilt in gleichem Maße auch für ehemalige Industrieflächen oder Hafenareale. Überall in Europa haben solche Brachen die Fantasie von kreativen Planern, Künstlern und Architekten beflügelt und sich für Kulturentwicklung und Kreativwirtschaft als förderlich erwiesen. Doch nicht immer finden diese Gentrifizierungs-Prozesse die Begeisterung der Bewohner (also der Studierenden, jungen Akademiker und Künstler), die jahrelang von der Vernachlässigung eines Quartiers profitiert haben und diese erst für den Markt in Wert gesetzt haben. Diese „Kreativen“ nutzen solche Räume in der Re- 
gel für eine Übergangszeit als Experimentierfeld für soziale und kulturelle Initiativen, bevor sie - nach ihrer „Entdeckung“ durch Stadtmagazine, Werbefotografen und „kreative“ Makler - ihre Verborgenheit verlieren und ihre Nutzer aufgrund steigender Mietkosten wieder ausziehen müssen.

Öffentliche Räume sichern: Auch wenn ein Großteil kulturellen Lebens in privaten Räumen stattfindet, sind für attraktive Städte doch öffentliche Räume die wichtigsten Orte kultureller Produktion und Konsumption. Kulturangebote in einer Shopping Mall können ebenso wenig wie die Ausstellungen in Schalterhallen von Sparkassen öffentliche Räume ersetzen. Solche Räume zu gestalten und ihre Gestaltung mit privaten Investoren abzustimmen, sie mit Leben zu füllen, das ist die Aufgabe kompetenter und unabhängiger Planer des öffentlichen Sektors. Öffentliche Räume haben darüber hinaus eine wichtige soziale Funktion in den europäischen Städten der Zukunft, da dort die oft beschworenen Face-to-Face-Kontakte stattfinden. Dort finden Menschen eine Bühne, wo sie sehen und gesehen werden, ohne Eintritt zahlen zu müssen. Für Unternehmen der Kreativ- und Kulturwirtschaft sind solche öffentlichen Räume unverzichtbar. Sie geben ihnen den dekorativen städtischen Rahmen, den sie brauchen, um sich zu entfalten und um Aufmerksamkeit zu finden.

Kulturwirtschaftliche Dimensionen in strategische Leitbilder der Stadtentwicklung integrieren: Die Formulierung räumlicher Leitbilder für Städte wie Regionen ist im Rahmen partizipativer Zielfindungsprozesse oder kreativer Zukunftswerkstätten in Deutschland wieder en vogue. Die Sicherung und Schaffung von Arbeitsplätzen dominiert verständlicherweise diese Leitbilder. Vermehrt werden dabei die Anforderungen innovativer Unternehmen und ihrer Beschäftigten oder auch der Tourismuswirtschaft berücksichtigt. Bei allem spielen lokale und regionale kulturelle Dimensionen eine große Rolle, insbesondere dann, wenn sie sich mit endogenen wirtschaftlichen Dimensionen verknüpfen lassen. Hier liegen die Ansatzpunkte für die Integration der Kreativ- und Kulturwirtschaft in räumliche Leitbilder. Sie bilden den strategischen Rahmen für konkrete lokale und regionale Maßnahmen.

Aus- und Fortbildungsgelegenheiten nutzen und ausbauen: Das mangelnde Interesse vieler Akteure an der Kultur hat auch damit zu tun, dass dieses Themenfeld im modernen Bildungskanon oft nur noch ein bedauerliches Schattendasein fristet. Das beruht auf sich ändernden gesellschaftlichen Wertesystemen, auf der ausufernden Diversifikation kultureller Angebote (,culture overkill“"), aber es liegt auch an dem immer größer werdenden Graben zwischen einer für breite Nutzergruppen leicht konsumierbaren kommerzialisierten Kunst und ihrer immer stär- 
keren und ohne Vorwissen kaum verständlichen Intellektualisierung. Während die Nutzung des Wissens von technischen, ökonomischen und medizinischen Hochschulen für die regionale Wirtschaft inzwischen zum Kern jeder Entwicklungsstrategie für die viel propagierte lernende Region gehört, stehen Kunsthochschulen noch immer im regionalen Schatten, weil sie kaum als kreative Laboratorien innovativer Entwicklung post-industrieller Gesellschaften angesehen werden. Aber auch die in der räumlichen Planung tätigen Fachleute werden im Rahmen ihrer Aus- und Fortbildung meist nicht mit der komplexen kulturellen Dimension ihres Handelns vertraut gemacht.

Auf Verbesserung der Rahmenbedingungen pochen: Viele Akteure, die in der Kulturwirtschaft unternehmerisch tätig sind, oder denen die Förderung der Kulturwirtschaft am Herzen liegt, wissen um die täglichen Hemmnisse. In den Kulturwirtschaftsberichten des Landes NRW wurde immer wieder darauf hingewiesen, dass die gesetzlichen, steuerpolitischen Rahmenbedingungen der Kulturwirtschaft optimiert werden müssen, wenn es gelingen soll ihre Produktionsbedingungen am Standort Deutschland zu verbessern. Solange aber die Kulturwirtschaft als Zukunftsbranche für eine im ökonomischen wie im kulturellen Sinne nachhaltige Raumentwicklung politisch vernachlässigt, und von den zersplitterten Interessenverbänden wenig Druck ausgeübt wird, dürfte die Motivation gering sein, die Rahmenbedingungen für kleine und mittlere Unternehmen kreativer Branchen zu verbessern.

\section{Fazit}

Kreativwirtschaft ist weltweit zu einem Handlungsfeld geworden, in das viele Städte neue Hoffnung setzen. Sie möchten es durch strategische Planung entwickeln und stärken, um bestehende Arbeitsplätze zu sichern und neue zu schaffen. Doch dies wird nur dort gelingen, wo alte mentale Barrieren abgebaut, wo Sektorpolitiken auf der lokalen und regionalen Ebene koordiniert werden können, wo wirtschaftliche, kulturelle und städtebauliche Politiken miteinender verknüpft werden können, um die endogenen Potenziale vor Ort zu nutzen - vielleicht auch um, was dann doch erheblich schwieriger sein wird, in kleinen Schritten neue Potenziale zu entwickeln.

Die große internationale Begeisterung für die Kreativwirtschaft wird die Städte in einen noch stärkeren weltweiten Wettbewerb um Events, Architekturmonumente und andere Bilder produzierende Projekte untereinander treiben. Auch wenn kulturwirtschaftliche Produkte, ähnlich wie Nahrungsmittel, immer wieder konsumiert werden, so wird es doch hier 
eine Sättigungsgrenze geben, über die hinaus ein privater Haushalt nicht mehr ausgeben wird. Aber auch der Bedarf von Unternehmen und Institutionen an Produkten und Dienstleistungen der Kreativwirtschaft ist nicht unbegrenzt.

Und je mehr Städte dieses Feld entdeckt haben und es mit öffentlichen Mitteln fördern, um so stärker wird der Wettbewerb um die Konsumenten und Nutzer sein, der den Städten dann neue Belastungen aufbürden, insbesondere dann, wenn ihre endogenen kulturwirtschaftlichen Potenziale begrenzt sind. Den weltweiten Trend zur Kreativwirtschaft zu nutzen ist sicher sinnvoll, aber Strategien zur Förderung der Kreativwirtschaft werden nur dann Erfolg haben, wenn sie lokal eingebunden und glaubhaft sind, wenn sie in Stadtpolitiken integriert werden. 



\section{Governance und Netzwerke in der Kreativwirtschaft}

BASTIAN LANGE

Der folgende Beitrag fragt, welche grundsätzlichen Perspektiven sich zwischen der Kreativwirtschaft und der Dimension Governance ergeben. Anders formuliert: Ist die Kreativwirtschaft steuerbar? Ziel des Beitrags ist die Qualifizierung des Verhältnisses zwischen Kreativwirtschaft und Governance. Ausgangspunkt dieser Frage sind verstärkte Aktivitäten der öffentlichen Hand (Bund und Kommunen), intermediärer Institutionen sowie der freien Wirtschaft, sich intensiver in das junge Handlungsfeld der Kreativwirtschaft einzubringen. Dies kann in Form von Imagepolitiken und Standortentwicklungen erfolgen oder mit dem Anspruch Marktpositionen neu auszurichten. Die Anwendung ist nicht folgenlos, da sie das historisch erkämpfte Terrain der Kulturpolitik systematisch in Frage stellt: Notwendig wird dies nicht zuletzt durch neue Produktionspraktiken und Neubewertungen vormals stabiler Professionshaltungen, wie bspw. dem Künstlertum.

Somit ergibt sich ein neuer Typus von Arbeitsorganisation, der sich empirisch im Gewand von kleinteiligen Netzwerkstrukturen und hochspezialisierten Nischenökonomien zeigt. Dieser beschreibt nicht nur den Wandel hin zu einem weitestgehend selbstbestimmten und ebenso entgrenzten Arbeits- und Produktionskontext. Er charakterisiert exemplarisch ein emergierendes und neu formierendes System, das der Kreativwirtschaft. Dieses gibt sich zunächst als ein paradoxes Konstrukt zu erkennen: Individuelles Autonomiebestreben steht im eklatanten Widerspruch zur Bedingung der Arbeit in sozialen Netzwerken, in denen ein hohes Mass an Vertrauen vorherrscht; lokal basierte Einbettungsmilieus 
stehen globalen Absatzmärkten gegenüber; etablierte Marktteilnehmer nehmen, um neue Produkte zu platzieren, im Marktgeschehen plötzlich die Rolle eines Außenseiters ein (DeFillippi/Grabher/Jones 2007: 511512; Lange/Kalandides/Stöber et al. 2008).

Die vehementen Veränderungen von Marktformierungen verweisen auf grundsätzlich neue Organisationsstrukturen und somit neue, reflexive Self-Governance-Formen (Kooiman 2003). Diese Formen sind situativ, ephemer und folgen eigenlogischen Regeln. Etablierte Fachverwaltungen, Verbände und andere Institutionen sind erst einmal irritiert und auf derartige Konstruktionen nicht eingestellt. In der Folge haben sozial-technologische Eingreifs- und Herrschaftsrhetoriken, im Sinne eines heroischen Managements wenig Chancen, adäquat in die Produktionsnetze dieser Kreativwirtschaft einzugreifen. Dennoch ist nicht von getrennten Wirkungs- und Funktionssphären zwischen der Arbeitswelt der Kreativwirtschaft einerseits und z. B. der öffentlichen Hand, der Politik oder der Zivilgesellschaft andererseits auszugehen: Vielmehr zeigen sich kontingente Sphären. An diesen Stellen wird der Bedarf nach neuen und zugleich tragfähigen Steuerungsperspektiven dringender denn je; wenn es gilt ein postheroisches Managementverständnis auf den Fall der Kreativwirtschaft anzuwenden (Baecker 1994).

Der folgende Beitrag erläutert, wie unter grundsätzlich neu zu bewertenden wissensgesellschaftlichen Rahmenbedingungen ein Begriff von Governance $\mathrm{zu}$ entfalten ist. Er nimmt die aktuellen heterogenen Organisationsprinzipien der Kreativwirtschaft zum Ausgangspunkt, um von dieser Fallsituation aus Perspektiven einer gelingenden Eingriffsund Steuerungspraxis überhaupt erst zu begründen. Im ersten Teil des Beitrags wird Kreativwirtschaft als Ausdruck eines neuen Modus von Arbeit und Innovation sowie neuen Interaktionsbeziehungen skizziert. Erklärungsbedürftig ist die sich daraus ableitende Governance- und Steuerungsdimension, um die in verschiedenen Funktions- und Gesellschaftsbereichen heftig gerungen wird. Die beobachtbaren Verfahrensweisen zur Profilierung des jungen Handlungsfeldes der Kreativwirtschaft werden sodann in Beziehung zu einem erweiterten Verständnis von Governance gesetzt. In einem zweiten Teil wird zunächst knapp der Begriff und das Konzept Governance vorgestellt und im dritten Teil daraus eine Sicht von Governance auf den Gegenstand Kreativwirtschaft angewandt. Die dabei vollzogene Erweiterung des Governance-Verständnisses weist somit den Weg, um neu über die Formierung von Wirkungszusammenhängen in der Kreativwirtschaft nachzudenken sowie daraus geeignete Steuerungsableitungen zu treffen. Das abschließende, vierte Kapitel setzt sich dann mit der Frage auseinander, wie das Arbeiten in offenen und changierenden Systemen und in schnell umbrechen- 
den Märkten nur auf der Grundlage von Improvisationen und situativen Strategien $\mathrm{zu}$ bewältigen ist und sich die Kreativarbeiter mit systematischen Unschärfen innerhalb offener Systemen auseinandersetzen müssen. Daraus ergeben sich Grundmaximen für neue Governance-Prozesse.

\section{Widerstreit um Governance-Formen der Kreativwirtschaft}

Um das Schlagwort des jungen Handlungsfeldes Kreativwirtschaft herum haben sich in jüngster Zeit vielstimmige und zugleich widerstreitende Diskurse angelagert, die oftmals einen nüchternen und zugleich sachlichen Blick nach der Frage ihrer Governance versperren. Governance wird hier als ein abgestimmter Modus verstanden, mit dem Akteure sich kommunikativ zu einem Markt bzw. innerhalb eines Marktes verhalten. Dabei lassen sich Regeln und Verfahrensweisen erkennen, um Preise und Bewertungen von symbolischen Gütern zu verhandeln (Lange/Mieg 2008). Der hier angewandte Begriff von Governance basiert auf einer neoinstitutionstheoretischen Perspektive, um nach den absichtsvollen Verfahrensweisen von Marktteilnehmern $\mathrm{zu}$ fragen (Mayntz/Scharpf 2005).

Er berücksichtigt aber ebenso die kommunikationsbasierte Perspektive, wie sie von der Systemtheorie (Willke 2007) hervorgehoben wird. Somit können einerseits die höchst eigenlogischen Regeln und Verfahrensweisen in den Blick genommen werden, wie sie in Teilbranchen der Kreativwirtschaft zu beobachten sind (Svejenova/Mazza/Planellas 2007; Haunschild/Eickhof 2007) sowie die sich daraus ergebenden sozialstrukturellen Formate, wie z. B. bestimmte soziale Netzwerke, innovative Milieus und kreative Szenen (Frey 2009; Lange 2007; Steets 2008). Die Produktion von symbolischen Gütern vollzieht sich meist in kleinteiligen und heterogenen Branchenstrukturen. Lange Zeit wurde dieses zentrale Merkmal der Kreativwirtschaft aus der Sicht etablierter Förderinstitutionen nicht anerkannt oder als Nachteil angesprochen.

Dieser Unübersichtlichkeit wird seit mehreren Jahren begegnet: Nach dem Vorbild Großbritanniens hat Nordrhein-Westfalen als erstes Bundesland in der Mitte der 1990er Jahre mit der Erarbeitung eines Berichts zum Status, der Struktur und der Relevanz der Kulturwirtschaft begonnen. Der dabei angewandte Branchenfokus verhalf dem Thema zu einer ersten umfassenderen Legitimation im (fach-)öffentlichen Bewusstsein. Seit wenigen Jahren zeichnen Kreativwirtschaftsberichte auf der Ebene der Städte, der Länder und ebenso des Bundes ein Wachs- 
tumsfeld. Neben der unbestreitbaren Relevanz erzeugen diese Berichte aufgrund der Fokussierung auf Wirtschaftsprozesse von Kultur bei Kulturpolitikern aber mitunter eine Abwehrhaltung: Sie befürchten die Instrumentalisierung sowie den Ausverkauf der öffentlich geförderten Kultur, oder, noch schlimmer, den Verlust der kulturellen Identität.

Der Widerstreit lässt sich aber nicht nur zwischen einem kulturpolitischen und einem marktwirtschaftlichen Lager aufzeigen. Ein weiterer Widerstreit gibt sich zwischen und innerhalb von Generationsmilieus zu erkennen. Gerade eine heute etablierte und in vielen Bereichen diskursführende Generation aus der Zeit des bundesdeutschen Wirtschaftswunders ist irritiert von den neuen und heterogenen Patchwork-Formen von Arbeitssituationen in der Kreativwirtschaft. Sie können nur schwer nachvollziehen, wie sich eine junge Generation anschickt, sich in der neuen, aus der Sicht der Traditionalisten, unbeständigen und unsicheren Arbeitswelt, einzurichten. Hat da eine Generation abseits etablierter Institutionen und Machtverhältnisse mit anderen Methoden und Absichten das erreicht, was die vorangegangene Generation nur durch den Gang der Institutionen und mit Hilfe der politischen Machtübernahme zu erzielen träumte? Hat die Generation der heute 25 bis 40-Jährigen, die in der Kreativwirtschaft tätig sind, strukturelle Autonomie in der Gestaltung ihrer Arbeitsbiographien erreicht?

Oder gilt nach wie vor, dass dies nur um den Preis der Illusion, der Annahme einer unwirklichen Welt, möglich ist? Folgt man der Sichtweise von Luc Boltanski und Eve Chiapello, so ist es in jüngster Zeit dem normativen System Kapitalismus gelungen, unter sich wandelnden Bedingungen wiederum Menschen zu gewinnen und davon zu überzeugen, sich am Prozess der kapitalistischen Akkumulation zu beteiligen: Eben in dem sie in der Kreativwirtschaft arbeiten. Grundlage war, dass dieser „Geist des Kapitalismus“ die gegen ihn gerichtete Kritik, wie sie seit den 1960er Jahren artikuliert wurde, konstruktiv verarbeiten konnte.

Die sich damals auflehnenden Künstler und Intellektuelle sind im Verlauf der letzten drei bis vier Dekaden mit ihren professionsspezifischen Attributen Flexibilität, Mobilität, Kreativität und Eigenverantwortung, die die heutige Beschäftigungsfähigkeit der Menschen bestimmen, ins bestimmende Zentrum gegenwärtiger Berufsfelder gerückt (Boltanski/Chiapello 2006; Koppetsch/Burkart 2002). Wer über diese Eigenschaften verfügt, kann die Möglichkeiten nutzen, die der projektbasierte Kapitalismus des 21. Jahrhunderts bietet.

So gesehen verwundert es nicht, wenn eine junge Generation ihr Glück in Form eines gelebten Pragmatismus und bezeichnenderweise im Projekt der „Marke Eigenbau“ (Friebe/Ramge 2008) sucht und suchen muss, um einen gangbaren Weg einzuschlagen, soziale, ökonomische 
und kulturelle Teilhabe zu erlangen. Paradox erscheint dieser Weg, weil diese Generation der Auffassung ist, in mikrokollektiven und netzwerkbasierten Arbeits- und Produktionsformen selbstbestimmt Nischen zu besetzen und einzig und allein in der Nischenproduktion eine Chance für gesellschaftliche Teilhabe sieht. Vielleicht ist dieser Weg jedoch gerade deshalb gangbar, weil mit der Formierung von unendlichen Schwärmen kleiner Projektnetze, so die Idee der Marke Eigenbau, die große neue Bewegung entstehen kann. Traditionelle Wege der Beeinflussung (finanzielles Kapital und/oder politische Macht) werden aus dieser Sicht nicht mehr als ein gangbarer biographischer Entwurf aufgefasst, um sich gesellschaftlich einzubringen.

Blickt man genauer auf diesen Selbststeuerungsmodus der Arbeit, so fällt die Sprunghaftigkeit und Labilität vieler arbeitsbiographischer Entwürfe auf. Sie lassen sich als ,situative Strategien“ (Seng 2008) ansprechen, mit der Protagonisten in changierenden Netzwerken Projekte bewältigen. Damit stellen sich für die Marktteilnehmer neue berufliche Anforderungen ein: Diese Anforderungen setzen sich aus dem Umgang mit offenen Systemen, mit systematischer Unschärfe, struktureller Ungewissheit sowie mit Komplexität zusammen: Improvisation ist angesagt (Dell 2003). Nichts prinzipiell Neues, so könnte man einwenden, auch andere wissensintensive Branchen müssen sich alltäglich mit instabilen Gewissheiten in ihrer Arbeitspraxis konstruktiv und proaktiv auseinandersetzen. Doch in den vom kreativem Schaffen geprägten Branchen ist das nicht die Ausnahme oder eine existentielle Bedrohung, vielmehr ist es originärer und konstitutiver Bestandteil der Arbeitsprozesse. Dieser reibt sich mit tradierten Modellen und Konzepten der Arbeitszeitführung, der Arbeitsorganisation und der Arbeitsteilung.

Wenn sich Arbeit in hochgradig funktional differenzierten Gesellschaften neu formiert und entlang von Kriterien wie Originalität, Authentizität und Flexibilität gestaltet, so ergeben sich auch Fragen nach der Steuerbarkeit der Kreativwirtschaft sowie nach grundsätzlichen Perspektiven im Spannungsfeld von Steuerungsanspruch, Steuerungswirkung sowie generell der Frage nach der Steuerbarkeit eines jungen Handlungsfeldes. Das Interesse leitet sich nicht zu letzt durch die vielfältigen Aktivitäten von z. B. Städten und Nationen ab, mit diesem Handlungsfeld Sichtbarkeit auf einer europäischen oder globalen Landkarte zu erzeugen.

Die marginalisierten Positionen von obligatorisch am Ende angefügten Handlungsempfehlungen in Kreativwirtschaftsberichten sind daher der Anlass, die Frage nach der Steuerbarkeit der Kultur- und Kreativwirtschaft in einen breiteren Kontext zu stellen. Dieser Kontext ist die Sicht der Organisationspraxis der zahlreichen selbstorganisierten Mikro- 
kollektive, Projektnetze und vorhandenen Steuerungsformen, die auf das Konzept Governance angewandt wird. Dazu bedarf es zunächst einer begrifflichen Einordnung des Begriffs und Konzepts Governance sowie einer Leistungsbeschreibung dieses Begriffs im Kontext und Anwendungsgebiet der Kreativwirtschaft.

\section{Governance: Begriff, Konzept - Anwendung auf die Kreativwirtschaft}

Lange Zeit wurde Governance im angelsächsischen Sprachbereich nur gelegentlich zur Bezeichnung des Prozessaspekts von Politik benutzt: governance bedeutete governing (Benz 2004). In den Wirtschaftswissenschaften lenkte der Begriff Governance die Aufmerksamkeit damit auf die Existenz von Regeln und die Art und Weise der Regeldurchsetzung im Wirtschaftsprozess (ebd.). Das in der Transaktionskostentheorie von Williamson eingeführte Begriffspaar Markt und Hierarchie wurde um die Dimension Netzwerke ergänzt (Powell 1990). Am Ende umfasste der Begriff Governance alle wesentlichen Formen der Handlungskoordination, rückte also die politische Dimension in den Hintergrund. Im Vordergrund steht die zustandegekommene Regelungsstruktur und ihre Wirkung auf das Handeln der ihr unterworfenen Akteure. Die Governance-Perspektive geht damit nahtlos in eine institutionalistische Denkweise über. Dadurch rücken Institutionen in den Vordergrund, die rationales Handeln über situative Anreize lenken.

Mit der Erweiterung des Governance-Begriffs auf Formen der Handlungskoordination jenseits von Markt und Hierarchie löste sich die Terminologie aus dem Bereich der Ökonomie und gewinnt so an Relevanz für neue Anwendungsfelder, wie das der Kreativwirtschaft. Governance kann sich sowohl auf eine Handeln regelnde Struktur als auch auf den Prozess der Regelung beziehen. Gleichwohl wird eine Anwendung dieses Governance-Begriffs auf Formen der mikrokollektiven SelfGovernanceformen, wie sie in der Kreativwirtschaft im Vordergrund stehen, selten praktiziert. Erst in jüngster Zeit erfolgt eine Übertragung des Begriffs Governance auf den Kreativwirtschaftskontext, z. B. als Ausdruck der Selbstorganisation von Kreativmilieus (Lange/Kalandides/Stöber et al. 2008).

In der hier geführten Diskussion wird daher der Blick weniger auf die politikwissenschaftliche Frage nach individuellen, sozio-ökonomischen oder kulturellen Dispositionen respektive der jeweiligen Kapitalformen und den daraus resultierenden Beiträgen hinsichtlich Planung, politischer Beteiligung oder Aktivitäten in demokratischen Verfahren 
gerichtet. Diese Festlegung erscheint aufgrund der fehlenden Stabilität und Belastbarkeit von kategorialen Begriffen wie z. B. Kollektiv und Kooperation für den Anwendungsfall Kreativwirtschaft nicht plausibel. Die für Governance-Fragen so wichtige Frage nach dem „Steuerungsobjekt", also der Frage, wie sich Akteure zu Kollektiven zusammenschließen, um dadurch ihre Interessen besser zu vertreten, erscheint für diesen Fall spannender denn je. Dies gilt vor allem für die projektorientierte Arbeitsweise, die weitaus situativere Zuschreibungen an „unvollständige Akteure" in zunehmend komplexeren und nicht abschließend zu überblickenden Ökonomien richtet (Priddat 2005). Nicht zuletzt dadurch wird die Anwendung etablierter politikwissenschaftlicher Konzepte auf den Fall Kreativwirtschaft erschwert, da sich der Gegenstand Kreativwirtschaft aufgrund seiner beobachtbaren eigenlogischen Praxis nicht so einfach in das Begriffs- und Analyseschema der bekannten GovernanceRhetoriken einfügt.

Aufgrund einer anderen Markteinführung symbolischer Produkte gibt sich eine fundamental andere Governance-Praxis $\mathrm{zu}$ erkennen: Unternehmerische Trägergruppen der Kreativwirtschaft zeichnen sich durch eigenlogische Strategien und Praktiken aus, um ihre Innovationen in Gestalt von symbolischen Gütern in kommunikativ hergestellte Netzwerksozialitäten hineinzufiltern und somit ihre produzierten Güter zunächst Bewertungskreisläufen auszusetzen (Lange/Mieg 2008). Erst die von anderen professionellen Akteuren (bestenfalls) als bewertungswürdig erachteten Güter haben sodann die Chance, wiederum in weiteren aufmerksamkeitsgenerierenden Funktionskreisläufen verhandelt zu werden.

Bei der Herstellung dieser Verkettungen spielen Vertrauen, Spezialistentum und Professionalität eine zentrale Rolle, ebenso das Spiel mit der Differenz. Markt ist daher zunächst ein performativer, verhandelbarer und dadurch sozial-räumlicher Gegenstand. Die dabei angelegten Netzwerke sind somit ein notwendiger Ausdruck von Marktgeschehen und werden nicht wie in klassischer Art entgegen Hierarchie oder als abgrenzbare „Gegenwelt“ zu Markt konzeptionalisiert, sondern sind im Sinne einer „marketization of networks“ zu verstehen (White 2002; Grabher 2004: 423).

Dieses Netzwerkverständnis sieht Macht, Spiel, Irritation, Camouflage und Performanz als Elemente der Herstellung von Netzwerken; Elemente also, die ganz wesentlich die Praxis eines schwer umkämpften und hochgradig risikobehafteten Marktes kennzeichnen. In diesen Formationen nimmt die Halbwertzeit der Gültigkeit von Innovationen, Wissen und Kennerschaft rasant ab. Der Druck auf die Herstellung von „Neuem“ ist hoch, mimetische Prozesse oder erkennbare Absatzbewe- 
gungen und Differenzierungsstrategien vom Mainstream verschaffen Aufmerksamkeitserträge und Autonomiegewinne, die aber gleichwohl stark risikobehaftet sind (McRobbie 2003; Ross 2008; Färber in diesem Band).

Bei den Netzwerken der Kreativwirtschaft ist also nicht von reinökonomischen Produktionsnetzwerken auszugehen, sondern vielmehr von gemischten Produktions-, Dienstleistungs- und Konsumentennetzwerken. Soziale Netzwerke sind sodann als neue "Suchpfade“ (Schindler 2006: 106) jenseits von traditionellen Hierarchien, etablierten Handlungspraktiken und politischen Verfahrensweisen zu begreifen.

Die gewachsene Bedeutung von Netzwerken in der ökonomiebezogenen Governance-Debatte wird durchgängig als eine Reaktion auf den Bedeutungsverlust nationalstaatlicher Steuerungsinstanzen gegenüber transnationalen Strukturen und Prozessen aufgefasst. Netzwerke werden entgegen ihrer „babylonischen Bandbreite“ (Börzel 1998: 254) als handlungsleitende Relevanzsysteme für individuelle Akteure und Interessenvertreter verstanden. Sie können im Fall der Kreativwirtschaft als Bindeglieder zwischen individuellen Dispositionen, politischen Felder und kreativen Märkten verstanden werden.

\section{Governance und Kreativwirtschaft - Ausgangspunkte}

Die wesentliche Arbeitspraxis in jungen Segmenten der Kreativwirtschaft wird durch die Dominanz von neuen Produktionsprozessen in schnell umbrechenden Netzwerken sowie temporärer Projektteams bestimmt. Dadurch stellt sich generell eine Situation ein, in der Unsicherheit und Unübersichtlichkeit maßgebliche Koordinaten der Bewältigung von Produktion darstellen. Aufgrund der Kurzfristigkeit der Projektarbeit sind die Projektmitglieder meistens hyperkomplexen Situationen ausgesetzt. Wie also ist in diesen Kontexten überhaupt ein Begriff von und eine Perspektive Governance zu platzieren? Nach Kooiman (2003) können diese Formen als Self-Governance angesprochen werden.

Richtet man den Blick aber auf die kommunikative Praxis der autonomen Formierung dieser jungen Marktsegmente, so eröffnet sich ein Blick auf einen weitaus komplexeren gesellschaftlichen Umbruch: Kreativwirtschaft ist ein heterogenes Handlungsfeld, das sich systematisch mit Komplexität und Unübersichtlichkeit auseinandersetzen und mit einem permanent vorhandenen Sinnüberschuss situative Lösungen erzielen muss. Dadurch liegt das Handlungsfeld Kreativwirtschaft nicht am 
gesellschaftlichen Rand, sondern ist als Labor für zukünftige Arbeitsund Produktionsformate anzusprechen.

In dieser dauerhaften Experimentierphase zeigt sich eine sogenannte Beta-Kultur (Neff/Stark 2003), d. h. die adhoc angesetzten Spielregeln sind immer suboptimal und fehlerbehaftet. Da es keinen „richtigen“ Steuerungsweg gibt, erscheinen neue Governanceformen als sogenannten postheroische Formen (Baecker 1994): Sie tendieren dazu hierarchische, monokausale, und lineare Ansätze zu vermeiden, um den komplexen Anforderungen gerecht zu werden. In iterativen Kommunikationsprozessen können aufkommende Steuerungsfehler besser aufgenommen und zügig bearbeitet werden.

Grundannahme einer derartigen Sichtweise auf Steuerung ist, dass man, um kontrollieren zu können, zuerst kontrolliert werden muss. Gerade postmoderne Varianten der Netzwerktheorie (White 2002) illustrieren die gegenseitigen Interdependenzen aller Steuerungsansätze in mehr oder weniger intransparenten Situationen. Die dabei entstehenden Koordinationsformen sind die Spiel- und Verfahrensregeln z. B. einer Teilbranche oder eines Teilmarktes, die sich durch bestimmte, kommunikativ errichtete Steuerungsformen überhaupt erst als Teilbranche/Teilmarkt zu erkennen geben.

\section{Ausblick oder Governance 2.0}

Die bisherige Argumentation basiert auf der Einschätzung, dass akteurszentrierte politikwissenschaftliche Governance-Ansätze zu rigide sind, um die beobachteten heterogenen Produktionspraktiken in der Kreativwirtschaft als Governanceformen zu konzeptionalisieren. Akteurszentrierten Ansätzen wohnt die Auffassung inne, dass „Kapazitäten“ durch kommunikative abgestimmte Regelverfahren in Kollektiven aufgebaut werden können, d. h. dass Steuerung per se einsehbar und somit durchführbar ist. Diese Auffassung entspricht somit einer Fortsetzung heroischer Ansätze, da sie an dem normativen Glauben festhalten, dass kollektives Handeln (,capacity building“) mit regional gebundenen Akteuren möglich ist. Dabei wird meistens die Idee aktualisiert, dass Kontrolle und Gewissheit über kollektives Handeln zu erzielen ist, d. h. dass Verhandeln und Organisieren von Zukunft trotz paradoxer Kontextbedingungen möglich ist. Inwiefern es aber plausibel ist, von homogenen Kollektiven zu sprechen, in denen abgestimmte Interessen vertreten werden, erscheint angesichts jüngerer Phänomene, die einerseits als crowdsourcing (1) und andererseits als social crowding (2) angesprochen werden, fragwürdig. 
(1) Unter dem Begriff des Crowdsourcing werden intellektuelle Produktionsprozesse in dezentrale transnationale Netzgemeinschaften ausgelagert, die als Schwarmintelligenz wiederum ihre Produkte in bestehende Unternehmensstrukturen einbinden. Crowdsourcing bzw. Schwarmauslagerung bezeichnet im Gegensatz zum Outsourcing nicht die Auslagerung von Unternehmensaufgaben und -strukturen an Drittunternehmen, sondern die Auslagerung auf die Intelligenz und die Arbeitskraft einer Masse von Freizeitarbeitern im Internet. Auf der Basis von webbasierten Interfaces können komplexe Projekte realisiert werden. Crowdsourcing kann auch als Form des Electronic Commerce stattfinden und wird in diesem Zusammenhang als Social Commerce bezeichnet. Dabei werden Kunden eines Anbieters zu persönlichen Filtern anderer Kunden und helfen diesen das bestmögliche Angebot zu finden. Beispiele für Crowdsourcing sind Wikipedia, OpenStreetMap und InnoCentive.

Diese Praxis funktioniert auch abseits großer Unternehmen im Rahmen selbstorganisierter Netzwerke wie etwa der Open Source-Bewegung. Produkte werden zu Gemeinschaftsleistungen, basieren regelrecht auf kooperativer Arbeitsteilung und komplexen Abstimmungsmechanismen. Strategien der gegenseitigen Bewertung und des Peerings machen die Vielfalt navigierbar, erschließen kollektives Wissen und legen neue Prozesse der Wertschöpfung frei. Kennzeichnend für die zugrunde liegenden hybriden Organisationsformen sind ein hoher Grad an Informalität, Fragmentierung und ständiger Transformationsbereitschaft der teilnehmenden Protagonisten.

(2) Entgegen der fehlenden Bodenhaftung dieser Schwärme gibt sich ein Trend zu erkennen, dezentrale und sozial vereinsamte Arbeitsweisen an Webinterfaces zu verlassen und sich wiederum in so genannten Coworking Spaces zusammenzufinden (siehe Lange/Wellmann in diesem Band). Die projektbezogene Arbeit verdichtet sich für eine bestimmte Zeit an einem sozialen wie gleichsam konkreten geographischen Ort. Diese sozialen Verdichtungen werden durch lokale Promotoren getragen, die darüber hinaus als Sozialstatiker den neu hinzukommenden Wissensarbeitern den Weg durch das neue Dickicht der Stadt weisen. Gleichwohl agieren sie neben ihrer Rolle als lokale Pfadfinder auch als Schnittstellenakteure zu anderen Projekten und vermitteln so zusätzlich Arbeit.

Erfolgreich könnte dieses Modell eines hybriden Café-ArbeitsKontextes zukünftig werden, weil es in der Lage ist, als Plattform und Schnittstelle zu anderen Projektnetzen freie Kooperation und kommerzielle Ausrichtung sowie Autonomiestreben und korporative Strukturen in einer Balance zu halten. Die Steuerung dieses Neben- und Miteinan- 
ders unterschiedlicher Kompetenzen und Geschwindigkeiten bedarf jedoch einer Governance-Praxis, die der Kurzfristigkeit und Heterogenität, den instabilen Gewissheiten und der immer post-demokratischeren Gestaltung des Marktgeschehens zu begegnen weiß. Wie in einem Ökosystem bedarf es dabei Mechanismen der Selbstregulierung und der Bereitstellung eines Raumes für die Emergenz verschiedener, in Relation zueinander stehenden (Un-)Ordnungssystemen.

\section{Fazit}

Die vorangegangenen Explorationen stellen eine Perspektive von aktuellen Formierungsprozessen in emergierenden Produktionsfeldern der Kreativwirtschaft dar. Sie sind als ein Plädoyer für hochgradig kontextspezifische und immer stärker netztechnologiebasierte Produktionsfelder zu sehen, in denen die Gewissheit ob des richtigen Steuerungsmodells, des marktgängigen Produktes und zielführender Investitionen exante kaum ermittelbar sind. Gewissheit in diesen offenen Systemen kann nur mittels einer Kultur der Improvisation, der Anerkennung der lokalen Situation als maßgebliche Begründungsdimension für wie auch immer geartetes Handeln in ständig changierenden Kontexten erreicht werden. In diesem Sinne ist die Kreativwirtschaft in einer Vorreiterrolle: Leadership im klassischen Sinne erhält nur Legitimation und Akzeptanz, wenn sich Führungsverantwortung im Verbund mit den Interessen von Mikrokollektiven einpendelt. Symbolische Innovationen sind daher nicht einzig einer Expertengruppe zuzuweisen oder von dieser zu erwarten, sondern im Grunde genommen nur durch vielfältige und nischenbasierte Schwärme zu erklären. Diese agieren als Resonanzboden und filtern Innovationen in weitere soziale Netzwerke.

Wendet man Governance als Ausdruck der Verfahrens- und Formierungsweisen von Marktteilnehmern in der Kreativwirtschaft an, so muss das Konzept Governance den folgenden Spannungsbogen aushalten: Den, der sich einerseits durch immer wirkungsmächtiger werdende Produktionskollektiven einstellt, die das Wohl eines wesentlichen Teils einer Branche bestimmen, und andererseits denen, die eine Form der spezifischen Eigenwilligkeit an den Tag legen, die wiederum den wesentlichen originären Kern kreativen Schaffens ausmacht. In diesem Spannungsverhältnis verortet sich der konstitutive Charakter der Kreativwirtschaft. Diese Eigenwilligkeit weist dann auch den Weg für individuelle, situative und überraschende Ansätze der z. B. öffentlichen Eingriffspraxis auf. Sie folgen keiner übergeordneten politischen Leitvorgabe, sondern bemessen sich an der Fähigkeit der Förderwilligen, die Eigenwilligkeit des Produktionsfeldes als etwas grundsätzlich Neues zu 
erkunden sowie anzuerkennen. Die Aufgabe besteht dann im Wesentlichen darin, sich im Treibsand der Produktionsschwärme immer wieder kurzfristig neu $\mathrm{zu}$ orientieren und unerwartete Überraschungen als Chance zu begreifen und nicht als Handlungshemmnis. Dazu bedarf es sicherlich einer robusten Praxistauglichkeit der Förderwilligen, die sich auf dem Weg in diese neuen Produktionswelten darauf einstellen sollten, dass sie durch das erfahrene Realitäts-Update nicht zu lange an vertrauten Positionen und bekannten Weltauffassungen festhalten.

\section{Literatur}

Baecker, Dirk (1994): Postheroisches Management: Ein Vademecum, Berlin: Merve-Verlag.

Benz, Arthur (2004): Governance - Regieren in komplexen Regelsystemen, Wiesbaden: VS-Verlag für Sozialwissenschaften.

Boltanski, Luc/Chiapello, Eve (2006): Der neue Geist des Kapitalismus, Konstanz: UVK-Verlagsgesellschaft.

Börzel, Tanja (1998): „Organizing Babylon - On the Different Conceptions of Policy Networks“. In: Public Administration, 76 (2), S. 253 274.

DeFillippi, Robert/Grabher, Gernot/Jones, Candace (2007): „Introduction to Paradoxes of Creativity: Managerial and OrganizationalCchallenges in the Cultural Economy“. In: Journal of Organizational Behavior 28 (5), S. 511-521.

Dell, Christopher (2003): „Improvisation braucht Methode. Sieben Takes“. Arch+ 167, S. 3-7.

Frey, Oliver (2009): Die amalgame Stadt: Orte. Netze. Milieus, Wiesbaden: VS-Verlag für Sozialwissenschaften.

Friebe, Holm/Ramge, Thomas (2008): Marke Eigenbau: der Aufstand der Massen gegen die Massenproduktion, Frankfurt/Main: Campus Verlag.

Grabher, Gernot (2004): „The markets are back!“ In: Progress in Human Geography 28 (4), S. 421-424.

Haunschild, Axel/Eickhof, Doris (2007): „For art's sake! Artistic and economic logics in creative production“. In: Journal of Organizational Behaviour 28 (5), S. 523-538.

Kooiman, Jan (2003): Governing as Governance, London: Sage.

Koppetsch, Cornelia/Burkart, Günter (2002): „Werbung und Unternehmensberatung als ,Treuhänder' expressiver Werte? Talcott Parsons' Professionssoziologie und die neuen ökonomischen Kulturvermittler.“ In: Berliner Journal für Soziologie 12 (4), S. 531-550. 
Lange, Bastian (2007): Die Räume der Kreativszenen. Culturepreneurs und ihre Orte in Berlin, Bielefeld: transcript Verlag.

Lange, Bastian/Kalandides, Ares/Stöber, Birgit, et al. (2008): „Berlin’s Creative Industries: Governing Creativity?“ In: Industry and Innovation 15 (5), S. 531-548.

Lange, Bastian/Mieg, Harald A. (2008): „Professionalisierungswege und Konstituierungen von ,Märkten" in den Creative Industries“. In: Geographische Zeitschrift 94 (4), S. 225-242.

Mayntz, Renate/Scharpf, Fritz (2005): Politische Steuerung - heute? Köln: MPIfG Working Paper 05/1, Januar 2005, Max-Planck-Institut für Gesellschaftsforschung, $12 \mathrm{~S}$.

McRobbie, Angela (2003): „I was knitting away day and night: Die Bedeutung von Kunst und Handwerk im Modedesign“. In: Osten, Marion von (Hg.), Norm der Abweichung, Zürich: Edition Voldemeer, S. 99-118.

Neff, Gina/Stark, David (2003): „Permanently Beta - Responsive Organisation in the Internet Era“. In: Howard, Philip E. N./Steve Jones (Hg.): The Internet and American Life, Thousand Oaks/CA: Sage, S. 173-188.

Powell, Walter W. (1990): „Neither Market nor Hierarchy: Network Forms of Organization“. In: Research in Organizational Behavior 12, S. 295-336.

Priddat, Birger P. (2005): Unvollständige Akteure: komplexer werdende Ökonomie, Wiesbaden: VS-Verlag für Sozialwissenschaften.

Ross, Andrew (2008): „The New Geography of Work: Power to the Precarious?“. In: Theory, Culture \& Society 25, S. 31-48.

Schindler, Delia (2006): „Die Rolle von Ideen und Deutungsmustern in der Politik: Wissenspolitologische Perspektiven auf Netzwerke“. In: Hollstein, Betina/Straus, Florian (Hg.): Qualitative Netzwerkanalyse, Opladen: VS-Verlag für Sozialwissenschaften, S. 99-123.

Seng, Judith (2008): „Ingredienzen der Projektgestaltung“. In: Kufus, Axel/Seng, Judith/Piesbergen, Mark et al. (Hg.): Design Reaktor Berlin, Berlin: Universität der Künste Berlin, S. 6-9.

Steets, Silke (2008): „Wir sind die Stadt!“: Kulturelle Netzwerke und die Konstitution städtischer Räume in Leipzig, Frankfurt/Main: Campus Verlag.

Svejenova, Silvia/Mazza, Carmelo/Planellas, Marcel (2007): „Cooking Up Change in Haute Cuisine: Ferran Adrià as an Institutional Entrepreneur". In: Journal of Organizational Behavior 28 (5), S. 539-561. 
White, Harrison C. (2002): Markets from networks: socioeconomic models of production, Princeton: Princeton University Press.

Willke, Helmut (2007): Smart Governance: Governing the Global Knowledge Society, Frankfurt/Main: Campus Verlag. 


\title{
Kreativwirtschaft und Metropolregionen - Konturen einer systemischen Steuerung
}

\author{
ALAIN THIERSTEIN, AGNES FÖRSTER, STEFAN LÜTHI
}

Aus dem Taumel der zahllosen Cluster-Projekte und Cluster-Strategien ist ein neuer Hoffnungsschimmer am Horizont von Standortförderung, Raumplanung, Politik und Beraterfirmen aufgetaucht: „Sei kreativ!“ Während man diese nutzlose Aufforderung früher Einzelpersonen zurief, gilt dieser gleiche Anspruch heute immer häufiger öffentlichen Körperschaften. Die kreative Stadt und der kreative Standort gelten als die magischen Attribute für Zukunftsfähigkeit. In diesem Wettlauf um die am besten qualifizierten und innovativsten Arbeitskräfte gelten gemeinhin Großstädte oder Metropolregionen als jene Konfigurationen mit den größten Anziehungsqualitäten - und Metropolitan Governance als das Mittel zum Zweck.

Die Herausgeber dieses Buchbeitrags haben uns die Aufgabe gestellt, den Zusammenhang zwischen der Förderung von Kreativwirtschaft und Metropolregionen $\mathrm{zu}$ untersuchen und daraufhin $\mathrm{zu}$ prüfen, auf welchem Wege sich daraus Aussagen für Governance ableiten lassen. Bestünde ein direkt beobachtbarer Zusammenhang, ließen sich unmittelbare Folgerungen für Governance formulieren. Unser Artikel geht von der These aus, dass es keinen - oder zumindest keinen direkten Zusammenhang zwischen der Förderung von Kreativwirtschaft und Metropolregionen gibt. Vielmehr wirkt die Kreativwirtschaft über ein vielgestaltiges System indirekt auf die Entstehung funktional polyzentrischer Metropolregionen.

Mit dieser These bedienen wir uns den zentralen Gedanken der Systemtheorie, welche eine isolierte Betrachtung einzelner Systembestandteile - wie z. B. eine isolierte Betrachtung der Kreativwirtschaft - ab- 
lehnt und demgegenüber eine ganzheitliche Perspektive bevorzugt. Eine systemische Betrachtungsweise trägt der Tatsache Rechnung, dass vieles zusammenhängt, was wir als getrennt erachten. Es liegt in der Natur eines Systems, dass jeder Teil so mit den andern Teilen verbunden ist, dass eine Änderung in einem Teil eine Änderung in allen Teilen und damit dem ganzen System verursacht. Ein System ist daher mehr als nur die Summe seiner Bestandteile; jeder Versuch einer Analyse künstlich isolierter Segmente würde das System als Gegenstand der Untersuchung zerstören (Watzlawick et al. 2000). Dies ist auch der Grund, wieso es unserer Meinung nach keinen Sinn macht, die Kreativwirtschaft als isoliertes Phänomen sondern als integrierter Bestandteil der Wissensökonomie zu betrachten, welche ihrerseits die treibende Kraft hinter dem Entstehen funktional verstandene polyzentrischer Metropolregionen ist. Dabei ist es nicht nötig, die einzelnen Systembestandteile bis ins kleinste Detail zu durchleuchten, denn so erfahren wir zwar vieles über diese Details, aber nichts über das System als solches. Aus diesem Grund argumentieren wir hier auf einem relativ hohen Aggregationsniveau. So werden wir nicht auf Differenzierungen innerhalb der Kreativwirtschaft oder der Wissensökonomie eingehen. Dies geschieht, um den Blick für das Gesamtsystem nicht zu verlieren und nicht aus Geringschätzung gegenüber der Relevanz für die aktuelle wissenschaftliche Debatte.

Bei einer systemischen Sichtweise steigt man aus dem zu betrachtenden System heraus, schaut von außen auf das eigene System und versucht dessen Verhalten zu erfassen und zu verstehen. Dabei versucht man zu eruieren, wo die kritischen, wo die puffernden Bereiche liegen, mit welchen Hebeln sich das System steuern lässt oder wo Symbiosemöglichkeiten oder Innovationspotenziale liegen (Vester 2007). Für die Governance von Kreativwirtschaft und Metropolregionen ist diese Sichtweise von entscheidender Bedeutung. Sie liefert den Entscheidungsträgern ein Modell, aufgrund dessen die ineinander greifenden vernetzten Abläufe transparent werden und auf deren Basis geeignete Impulsvorgaben zur Selbstregulation des Systems abgeleitet werden können. Wo immer man auch eingreift, pflanzt sich die Wirkung fort, verliert sich oder wirkt auf Umwegen wieder zurück. Um zu erfassen, was verschiedene Governanceformen in einem komplexen System bewirken, kommt man nicht umhin, das Muster seiner vernetzten Dynamik besser verstehen zu lernen (Vester 2007).

Basierend auf dieser systemischen Betrachtungsweise stellt dieser Buchbeitrag zunächst die Klärung einzelner Begriffe und Konzepte in den Mittelpunkt. Kreativwirtschaft und Metropolregionen zusammen zu denken gelingt nur, wenn Kreativwirtschaft nicht isoliert, sondern als ein Teil der Wissensökonomie betrachtet wird. In einem ersten Schritt ver- 
suchen wir darum die Konturen des Untersuchungsgegenstands über die Definition der wesentlichen Begrifflichkeiten zu skizzieren. Darauf aufbauend leuchten wir im zweiten Teil das Phänomen Kreativwirtschaft und Metropolregion von zwei verschiedenen Seiten aus. In der Nachfragerperspektive erscheint die Kreativwirtschaft als Nachfrager von Standortqualitäten in Metropolregionen; in der Angebotsperspektive wird Kreativwirtschaft als Standortfaktor von Metropolregionen betrachtet. Das Zusammenspiel von Kreativwirtschaft und Metropolregionen lässt sich nun als eine Überlagerung geografischer, institutioneller und sozialer Nähe auf verschiedenen Maßstabsebenen erkennen. Im dritten Teil diskutieren wir Möglichkeiten und Grenzen der Steuerbarkeit von Kreativwirtschaft und Metropolregionen und stoßen dabei auf zwei mögliche Konzepte für die Umsetzung einer systemischen Steuerung: der Triple Helix Ansatz und das Konzept von räumlich-funktionalen Komplementaritäten.

\section{Kreativwirtschaft, Wissensökonomie, Metropolregionen - Was ist darunter $\mathrm{zu}$ verstehen?}

Wissensorientierte Wirtschaftstätigkeiten, die man gemeinhin unter dem Begriff der Wissensökonomie zusammenfasst, gewinnen an Bedeutung. Die statistische Abgrenzung der Wissensökonomie ist kein leichtes Unterfangen. In empirischen Untersuchungen gibt es eine Vielzahl von Abgrenzungen. Die Unterscheidung zwischen wissensintensiven, forschungsintensiven und kreativen Wirtschaftszweigen ist eine Möglichkeit, die zahlreichen Definitionen zu strukturieren. Wissensintensive Wirtschaftszweige charakterisieren sich durch eine hohe Akademikerquote und durch eine große Anzahl an Forschungs-, Entwicklungs- und Konstruktionspersonal (Legler/Frietsch 2006). Forschungsintensiv sind diejenigen Wirtschaftszweige, welche eine hohe Forschungs- und Entwicklungsintensität sowie eine hohe Wissenschaftlerintensität aufweisen (Legler/Frietsch 2006).

Als kreativ können Unternehmen bezeichnet werden, ,die sich auf erwerbswirtschaftlicher Basis mit der künstlerischen/kreativen Produktion, ihrer Vermittlung und/oder medialen Verbreitung von entsprechenden Gütern und Dienstleistungen befassen“ (Held/Kruse 2005: 7). Konkret subsummiert Richard E. Caves (2000) darunter Bereiche wie „,book and magazine publishing, the visual arts (painting, sculpture), the performing arts (theatre, opera, concerts, dance), sound recordings, cinema and TV films, even fashion and toys and games" (Caves 2000: 1). Ri- 
chard Florida (2002) zählt auch Architekten und Designer sowie Ärzte, Wirtschaftsprüfer und Unternehmer dazu (Florida 2002). Gemäß dem so genannten Drei-Sektoren-Modell existieren neben der privatwirtschaftlich ausgerichteten Kreativwirtschaft, welche sich nach einer eigentlichen Branchenlogik definiert, noch zwei andere Bereiche, welche kulturelle Leistungen anbieten: Zum einen ist es der Staat, welcher basierend auf gesetzlichen Grundlagen Kunst und Kultur fördert und die kulturelle Grundversorgung garantiert. Zum anderen sind es gemeinnützige Vereine und Organisationen, welche weitere kulturelle Leistungen zur Verfügung stellen (Söndermann et al. 2008). In diesem Artikel konzentrieren wir uns explizit auf den privatwirtschaftlichen Bereich der Kreativwirtschaft.

Abbildung 1 zeigt die direkte Gegenüberstellung der Definitionen von forschungsintensiven, wissensintensiven und kreativen Wirtschaftszweigen. Die Abgrenzungen basieren auf den empirischen-analytischen Definitionen von Legler/Frietsch (2006) bzw. Held et al. (2005). Die Größe der Kreisflächen illustriert die Anzahl der Wirtschaftszweige in den entsprechenden Branchen. Die unscharfe Abgrenzung der Kreise macht deutlich, dass auch innerhalb der einzelnen Bereiche unterschiedliche Definitionen verwendet werden. Innerhalb der „Schnittmengen“ ist die absolute Anzahl der überlagernden Wirtschaftszweige abgebildet. Auffallend ist, dass forschungsintensive Wirtschaftszweige vollständig in den wissensintensiven enthalten sind, und dass gut die Hälfte der kreativen Wirtschaftszweige auch als wissensintensiv definiert wird.

Unser Artikel verknüpft Kreativwirtschaft mit dem Phänomen der Metropolregion, für die keine klaren Vereinbarungen zur Definition und Abgrenzung dieser Raumeinheit besteht (Adam/Göddecke-Stellmann 2002). In der deutschen Metropolregionsdiskussion dominieren drei unterschiedliche Lesarten, welche zwar komplementär, bei Weitem aber nicht deckungsgleich sind: die politisch-normative, die analytischfunktionale und die räumlich-morphologische Perspektive.

Die politisch-normative Perspektive. Aus politisch-normativer Sicht werden Metropolregionen als Leitvorstellung der Raumentwicklung definiert. Damit soll die internationale Wettbewerbsfähigkeit eines Landes erhöht und das nationale Wachstum gestärkt werden. Vor diesem Hintergrund wurden bereits im Jahre 1995 von der Ministerkonferenz für Raumordnung (MKRO) im Rahmen des raumordnungspolitischen Handlungsrahmens sieben deutsche Metropolregionen in ihrer Bedeutung hervorgehoben. Bis 2006 sind weitere vier Metropolregionen dazugekommen (MKRO 1995). Im ersten Leitbild „Wachstum und Innovation“ der „Leitbilder und Strategien für die Raumentwicklung in Deutschland“, die im Juni 2006 von der Ministerkonferenz für Raum- 
ordnung verabschiedet wurden, spielen die Metropolregionen eine herausragende Rolle (MKRO 2006). In diesem Leitbild wird ein „,weiter metropolitaner Verflechtungsraum“ flächendeckend über ganz Deutschland gezogen, so dass keine weißen Flecken auf der Landkarte entstehen und sich jede Raumeinheit an eine Metropolregion angeschlossen fühlen darf. Damit versucht man dem politisch-normativen Anspruch der „Gleichwertigkeit der Lebensverhältnisse in allen Teilräumen“ (Bundestag 1998) gerecht zu werden.

Abbildung 1: Vergleich von wissensintensiven, forschungsintensiven und kreativen Wirtschaftszweigen

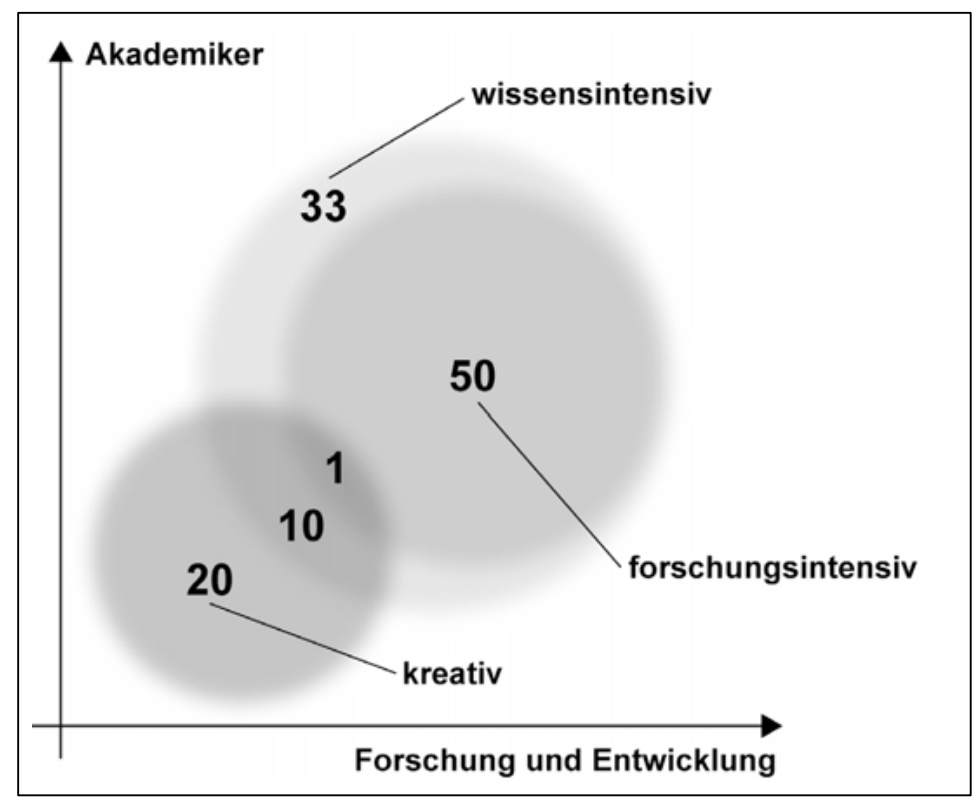

Quelle: eigene Berechnung und Darstellung

Die analytisch-funktionale Perspektive. Im gegenwärtigen wissenschaftlichen Diskurs dominieren zwei analytische Konzeptionen. Die erste Konzeption bezieht sich auf die Funktion von Metropolregionen, die sie im Rahmen des zunehmenden globalen Standortwettbewerbes und der Standortanforderungen der Wissensökonomie erbringen (Bonneville 1994; Blotevogel 2002). In dieser Konzeption werden Metropolregionen als eine Art Behälter verstanden, die spezifische Metropolregionsfunktionen beherbergen, wie Entscheidungs- und Kontrollfunktionen, Innovations- und Wettbewerbsfunktionen, Gateway- und Symbolfunktionen (Blotevogel 2002; Schmitt 2006). Die überwiegende Zahl dieser Studien 
basiert auf Attributdaten, wie beispielsweise der Zahl von Unternehmenssitzen oder internationalen Organisationen, dem Volumen des Börsenhandels oder der Kapazität technologisch-wissenschaftlicher Infrastruktur. Die wesentliche Schwäche dieses Ansatzes besteht darin, dass man dem Diktat sekundärstatistischer Attributdaten verhaftet bleibt und dadurch keine Aussagen über relationale Beziehungen zwischen Standorten machen kann.

Im Gegensatz zur ersten trägt die zweite analytisch-funktionale Konzeption der Tatsache Rechnung, dass räumliche Entwicklung ein Ergebnis sozialer Interaktionen ist und auf Relationen zwischen wirtschaftlichen Akteuren basiert. Metropolregionen werden nicht als Behälter von spezifischen Funktionen gesehen, sondern als Prozessraum, welcher über funktionale Verflechtungen definiert wird. Solche funktionalen Verflechtungen können zum einen physische Verflechtungen wie Pendlerbeziehungen oder internationale Flugverbindungen sein, zum andern aber auch nicht-physische Verflechtungen wie Unternehmensbeziehungen oder elektronische Finanztransaktionen.

In dieser relationalen Perspektive übernehmen Metropolregionen Knotenfunktionen für global vernetzte Güter-, Kapital-, Informationsund Personenströme (Castells 1996; Friedmann 1986; Sassen 2001; Taylor 2004; Kujath 2002). Manuel Castells' Network Society (1996) ist eine der einflussreichsten Arbeiten in diesem Zusammenhang. Seine Gedanken haben eine neue Wahrnehmung von Raum und räumlicher Entwicklung stimuliert. Mit seinem Konzept des „Space of Flows“ macht er deutlich, dass sich unsere Gesellschaft rund um Ströme errichtet: Kapitalströme, Informationsströme, Technologieströme, Ströme organisatorischer Interaktion, Ströme von Bildern, Klängen und Symbolen (Castells 1996: 412).

Peter Taylor's (2004) Modell des „World City Network“ operationalisiert diese Idee, indem es die Beziehungen zwischen Stadtregionen mit Hilfe der Organisationsstrukturen global agierender Firmen analysiert (Taylor 2004). Im EU-Forschungsprojekt POLYNET wurde diese Methodik auf die Maßstabsebene von Metropolregionen angewendet und zum Vergleich von acht Beispielregionen in Westeuropa herangezogen (Hall/Pain 2006b). Im POLYNET Projekt wurden Metropolregionen als „Mega-City Regions“ bezeichnet und folgendermaßen definiert: „MegaCity Regions are a series of anything between 10 and 50 cities and towns physically separated but functionally networked, clustered around one or more larger central cities, and drawing enormous economic strength from a new functional division of labour" (Hall/Pain 2006: 3). Im vorliegenden Beitrag bauen wir auf dieser Definition der Metropolregion auf. Wir beziehen uns damit explizit auf ein relationales und ana- 
lytisch-funktional definiertes Verständnis von Metropolregionen und ergänzen damit die politisch-normative Dimension durch eine entscheidende Komponente.

Die räumlich-morphologische Perspektive. Aus räumlich-morphologischer Sicht können Metropolregionen als polyzentrische Räume angesehen werden, die hoch verdichtete städtische Räume, semi-rurale Gegenden sowie urbane Kulturlandschaften umfassen. Wissensbasierte Unternehmen suchen gezielt Orte mit besonderem „Look and Feel“, denn sie brauchen zur Leistungserstellung nicht nur Kontakte zu Kunden, Zulieferern und anderen Dienstleistern, sondern auch bestimmte Qualitäten der räumlichen Umgebung, damit Kreativität und Schaffensdrang ungehindert fließen können (Helbrecht 2005: 144).

Die Rolle von Metropolregionen als funktionale Knoten im globalen „Space of Flows“ wie auch die Bedeutung von atmosphärischen Standort- und Gestaltqualitäten ergänzen sich als Standortfaktoren gegenseitig. So berichtet Helbrecht (2005: 145), dass die kreativen Dienstleister in München und Vancouver in ihren jeweiligen Städten, Stadtteilen, Straßenzügen und Büroräumen eine ausgewogene Balance aus der Nähe zu Kunden, Zulieferern und Kooperationspartnern wie auch Wohlfühlräume für den geistigen Schöpfungsprozess suchen. Ihr Erfolg gründet sich - neben den Netzwerkökonomien - zu einem wesentlichen Teil auf einer dezidiert subjektiven Wahrnehmung und Bewertung der erlebten Stadtlandschaften und ihren Bildern (Helbrecht 2005).

Mit diesen drei unterschiedlichen Lesarten von Metropolregionen im Hinterkopf, wollen wir im Folgenden das systemische Zusammenspiel von Kreativwirtschaft, Wissensökonomie und Metropolregionen aufzeigen und dessen verschiedenen, räumlichen Artikulationen herausarbeiten und diskutieren.

\section{Kreativwirtschaft, Wissensökonomie, Metropolregionen - ein systemisches Zusammenspiel}

Während die allmähliche Herausbildung von polyzentrischen Metropolregionen wesentlich auf der funktionalen nicht-physischen räumlichen Ebene, also unterhalb der Schwelle unserer Wahrnehmung verläuft, ragen uns deutliche physische Landmarken entgegen: Fußballstadien, Hochhäuser, Kunstmuseen, Forschungsinstitute, Flughäfen und Autostädte. Ihre bildhafte Wirkung schlägt sich real, medial und mental nieder (Förster/Thierstein 2008). Wissensökonomie und Kreativwirtschaft zeigen sich in gut sichtbaren Orten innerhalb von Metropolregionen in 
Nachbarschaft zueinander: Central Business District und pulsierendes kreatives Bahnhofsviertel, Hightech Campus und Medienstadt. Diese sichtbare Nachbarschaft lässt sich erklären als Resultat eines systemischen Zusammenspiels von Kreativwirtschaft und Wissensökonomie in verschiedenen Raumdimensionen und auf verschiedenen räumlichen Maßstabsebenen.

Die wesentliche Verknüpfung zwischen Kreativwirtschaft und Wissensökonomie ist dabei auf der nicht-physischen funktionalen Ebene anzusiedeln. Kreativwirtschaft und Wissensökonomie stehen als Geschäftspartner und Kunden im gegenseitigem Austausch. Diese Geschäftsbeziehungen können dabei innerhalb von Unternehmen zwischen verschiedenen Standorten und Geschäftsfeldern oder zwischen verschiedenen Unternehmen ähnlicher oder verschiedener Branchen bestehen. Kreativwirtschaft und Wissensökonomie sind dabei durch wechselseitige Wertschöpfungsbeziehungen miteinander verknüpft. Diese funktionalen Austauschbeziehungen manifestieren sich in Informations- und Kommunikationsströmen, sind also immaterieller, nicht physischer Natur. Die Verräumlichung dieses netzwerkartigen Zusammenspiels ließe sich mit ähnlichen analytischen Verfahren bewerkstelligen, wie sie in dem europäischen Forschungsprojekt POLYNET für die Wissensökonomie in den Metropolregionen Nord-West Europas angewendet wurden (Hall/Pain 2006b). Das Ergebnis würde zeigen, welche räumlichen Maßstabsebenen für die Konstitution der Austauschbeziehungen zwischen Kreativwirtschaft und Wissensökonomie von strategischer Bedeutung sind. Eine Antwort könnte lauten: Metropolregionen.

Da solche Forschungsergebnisse zumindest den Autoren dieses Artikels nicht vorliegen, nähern wir uns der Verräumlichung des funktionalen nicht-physischen Zusammenspiels von Kreativwirtschaft und Wissensökonomie im Folgenden mit einer indikativen Vorgehensweise an. In die wechselseitige Betrachtung von Angebot und Nachfrage zwischen den beiden Wirtschaftsfeldern beziehen wir weitere Raumdimensionen ein. Wir betrachten die Standortfaktoren, über die Kreativwirtschaft und Wissensökonomie miteinander verbunden sind. Unter funktionalen Standortfaktoren verstehen wir die räumliche Ausstattung mit physischen Infrastrukturen, Institutionen und Arbeitskräften; auf der morphologisch räumlichen Ebene sind städtebauliche und landschaftliche Strukturen und Qualitäten bedeutend; zur Ebene des Bilds und der Wahrnehmung gehören sowohl innere Vorstellungsbilder, Images und Reputation wie auch real wahrnehmbare städtische Atmosphären und Bilder. Die näherungsweise Verräumlichung dieser angebotenen und nachgefragten Standortfaktoren lässt Schlüsse über die Rolle und das Zusammenspiel verschiedener räumlicher Maßstabsebenen zu. 


\section{Nachfrageperspektive - Kreativwirtschaft als Nachfrager von Standortqualitäten}

Kreative Unternehmen und kreativ tätige Menschen sind ein anspruchsvolles Klientel. Der weltweite Wettbewerb um Talente ist in aller Munde (Michaels/Handfield-Jones/Axelrod 2001). Auch für die Kreativwirtschaft ist das Vorhandensein einer kritischen Masse an qualifizierten, kreativen Arbeitskräften ein wesentlicher Standortfaktor. Dabei sind kreative Talente häufig auch Unternehmer. Die Kreativwirtschaft besteht in Deutschland und der Schweiz mehrheitlich aus Klein- und Kleinstunternehmen, die Quote der Selbständigen und Freiberufler in diesen Beschäftigungszweigen ist überdurchschnittlich hoch (Söndermann et al. 2008; Referat für Arbeit und Wirtschaft 2007).

Für die Stadt München zeigt der Vergleich der Standortanforderungen von hoch kreativen Berufsgruppen und hoch qualifizierten Wissensarbeitern deutliche Unterschiede hinsichtlich der Anforderungen an das Wohn- und Arbeitsumfeld sowie das Freizeitangebot. So bevorzugen die Hochkreativen deutlich nutzungsgemischte innenstadtnahe Quartiere, die hoch qualifizierten Wissensarbeiter hingegen fragen gleichermaßen Wohnstandorte in der Innenstadt wie am Stadtrand nach (Referat für Arbeit und Wirtschaft 2007). Die Untersuchung des Standortwahlverhaltens von Unternehmen der Kreativwirtschaft innerhalb der Stadt Zürich bestätigt die herausragende Bedeutung des Faktors „Urbanität“. Sowohl auf kantonaler wie auch auf städtischer Ebene zeigen sich räumliche Konzentrationsprozesse der Kreativwirtschaft, welche auf urbane Räume und darin wiederum auf bestimmte zentrale Stadtquartiere abzielen (Söndermann et al. 2008). Räumliche Nähe ist ein gefragtes Gut. Folgende Lesarten bieten sich an:

Kreativwirtschaft fragt Kreativwirtschaft nach. Die Kreativwirtschaft unterliegt speziellen Werthaltungen, Denk- und Arbeitsweisen, welche sie von anderen Wirtschaftsbranchen unterscheidet. Die spezifische Logik der Ökonomie der Kreativwirtschaft lässt sich in Teilen mit dem Konzept der Embeddedness - Einbettung - erklären. Ökonomische Interessen können nur zum Teil das Akteursverhalten in der Kreativwirtschaft erklären. Für die Arbeitsprozesse der Kreativwirtschaft sind Reputation, der Austausch in informellen Netzwerken und wenig vertraglich abgesicherte Kooperations-, Zulieferer- und Kundenbeziehungen prägend. In diesem kreativen Milieu spielen Vertrauen, häufig jahrelang aufgebaut, gemeinsame Ideengenerierung und Schlüsselpersonen eine entscheidende Rolle (Aemisegger 2007; Held/Kruse 2005). Das berufliche Netzwerk ist entscheidend für die Gewinnung von Kunden und Geschäftspartnern und die Reduktion des Risikos der Arbeitslosigkeit (Re- 
ferat für Arbeit und Wirtschaft 2007). Der Standortfaktor räumliche Nähe liest sich für die Kreativwirtschaft als soziale Interaktion in dichten, kreativen Quartieren oder campusartigen städtebaulichen Anlagen. Dabei überlagern sich Produktion und Konsum von Kreativwirtschaft. Für die Kreativbranche selbst sind die Möglichkeiten und der Zugang zu kulturellen Angeboten, Trends, Moden, technologischen und gesellschaftlichen Entwicklungen wichtig zur Aneignung von aktuellem Wissen sowie als Inspirationsquelle. Die Kreativen fragen selbst ein kulturelles Angebot und ein kreatives Milieu nach (Söndermann et al. 2008; Referat für Arbeit und Wirtschaft 2007). Die enge Verknüpfung des öffentlichen, intermediären und privaten Sektors der Kreativwirtschaft ist in zahlreichen Biografien nachweisbar (Held/Kruse 2005; Weckerle/Söndermann 2005). Der privatwirtschaftliche Teil der Kreativwirtschaft sucht die räumliche Nähe zu kreativen öffentlichen Einrichtungen wie Hochschulen, Museen oder Opernhäusern.

Kreativwirtschaft fragt Kunden nach. Die Kreativwirtschaft stellt hochwertige Produkte und Dienstleistungen her. Anspruchsvolles Angebot sucht anspruchsvolle Nachfrage (Porter 1990). Die Kunden der Kreativwirtschaft - Unternehmen oder private Endverbraucher - sind selbst häufig in wertschöpfungsintensiven Branchen tätig. Sassen (2001) beschreibt, wie die hoch bezahlten Wissensarbeiter in Global Cities wie New York oder London in besonderem Maße personennahe Dienstleistungen nachfragen, um bei hoher Mobilität und knappen zeitlichen Ressourcen ihre Ansprüche im alltäglichen Leben zu befriedigen (Sassen 2001). Auf ähnliche Art und Weise bewegen sich besonders die Hersteller der Simple Creative Goods (Caves 2000), dazu zählen Produkte und Dienstleistungen mit nur geringer Arbeitsteilung und hohem Einsatz von beinahe handwerklichem Erfahrungswissen, im Windschatten von gut verdienenden hoch qualifizierten Menschen und von Unternehmen mit überdurchschnittlicher Wertschöpfung (Caves 2000). Räumliche Nähe $\mathrm{zu}$ einer ausreichend kritischen Masse von anspruchsvollen Kunden ist für Kreativwirtschaft ein entscheidender Standortfaktor.

Kreativwirtschaft fragt Wertschöpfungsbeziehungen nach. Rückt man die Complex Creative Goods in den Mittelpunkt der Betrachtung, dazu sind unter anderem Filme, Bücher und Musikaufnahmen zu zählen, welche in ausdifferenzierten Teams entstehen, zeigt sich, dass die Kreativwirtschaft ebenso wie viele andere wissensintensive Branchen hochgradig arbeitsteilig organisiert ist (Caves 2000). Die Arbeitsteilung besteht zwischen den verschiedenen Teilbereichen des privatwirtschaftlichen kreativen Sektors und zu anderen Wirtschaftszweigen wie unternehmensnahe Dienstleistungen, Tourismus, Freizeit, Sport und Wellness. In Zürich zeigt sich der Finanzplatz als zentraler Nachfrager für 
kreative Dienstleistungen (Held/Kruse 2005). Kreative Produkte sind hier Teil umfassender Wertschöpfungsketten. Gerade die Wissensökonomie zeichnet sich durch eine kulturelle Durchdringung von Produkten und Dienstleistungen aus. Dafür bieten kreativ tätige Unternehmen strategische Bausteine an (Held/Kruse 2005; Caves 2000). Die milieuspezifischen Standortanforderungen der Kreativen sind nun eingebettet in die Standortanforderungen der Wissensökonomie zu verstehen.

\section{Angebotsperspektive - Kreativwirtschaft als Standortfaktor}

Kreativwirtschaft ist ein Standortfaktor - doch mit welcher Kraft? Wir wollen zunächst die Wirkungsebenen verstehen.

Kreativwirtschaft bietet Produkte und Wissen an. Der privatwirtschaftliche, intermediäre und auch öffentliche Sektor der Kreativwirtschaft stellen Kulturangebote, künstlerisch-kreative Produkte und Dienstleistungen bereit. Kulturelle Zeichen, Symbole und Designs sind zunehmend Bestandteil der Wirtschaft. In der Wissensökonomie hat die Kreativwirtschaft eine wichtige Interface-Funktion, indem sie Dienstleistungen und Produkte bereitstellt, welche im weitesten Sinne der Kommunikation zwischen Unternehmen und Kunden dienen. Insofern kann die Verfügbarkeit hoch qualitativer kreativer Produkte für bestimmte Wirtschaftszweige einen relevanten Standortfaktor darstellen. Daneben sind kulturelle Angebote und Institutionen für Städte und Regionen von erheblichem Freizeit- und Tourismuswert.

Kreativwirtschaft bietet Stadträume an. Die Akteure der Kreativwirtschaft schaffen sich ihr Umfeld, das sind Mitarbeiter, Infrastrukturen, Netzwerke. Dabei produzieren sie städtische Räume: Zwischennutzungen in Industriebrachen und Kasernen oder trendige, kreative Arbeiterquartiere. Kreative Menschen und Unternehmen sind vielfach die Vorhut - Pionierpflanzen oder Trend Scouts - einer Spirale der Aufwertung von städtischen Lagen und Liegenschaften. Sie scheinen räumliche Qualitäten in besonderer Weise aufzuspüren und herzustellen. Die entstehenden Angebote sind neu und überraschend, sie treffen vielfach den Nerv des Immobilienmarkts.

Kreativwirtschaft bietet Sichtbarkeit an. Kreative Menschen und Unternehmen produzieren Bilder, Zeichen, Symbole für ihre Kunden. Die Summe dieser einzelnen kreativen Aktivitäten strahlt aus und prägt auch die Atmosphäre und Reputation des Quartiers, der Stadt - auch der Metropolregion? Metropolregionen entziehen sich unserer unmittelbaren Wahrnehmung. Immaterielle funktionale Austauschbeziehungen zwischen Unternehmen, also nicht sichtbare Informations- und Kommuni- 
kationsströme, stehen im Zentrum der analytischen Betrachtung. Thierstein et al. sprechen daher von „Raumentwicklung im Verborgenen“ (Thierstein et al. 2006). Die räumlich weit ausgreifenden normativplanerischen Gebilde sprengen gewohnte Raumkategorien. Ihre städtebaulich-landschaftliche Morphologie lässt keinen zwingenden strukturellen Zusammenhang erkennen (Förster/Thierstein 2008). Vor dem Hintergrund dieser Unsichtbarkeit wirbt die Metropolregion Hamburg mit der Elbphilharmonie. Berlin gilt als arm aber sexy, das manifestiert sich in einzelnen Straßenzügen und Stadtteilen und strahlt bis nach Brandenburg aus. Die Orte der Kreativwirtschaft gehen in die Prospekte von Metropolregionen ein. Die kulturellen Leuchttürme und kreativen Hot Spots der Kernstädte sollen helfen, die gesamte Metropolregion zu kommunizieren, also auch die Stadtlandschaften oder semi-ruralen Räume außerhalb der urbanen Zentren. Kreativität und Kultur bergen den Reiz des Sichtbaren und dienen den institutionell gefassten Metropolregionen in Deutschland als visuelle Anker und Identitätspunkte.

\section{Fazit}

Sowohl auf Nachfrageseite, wie auch auf Angebotsseite ist Kreativwirtschaft eng verflochten mit der Wissensökonomie. Abbildung 2 zeigt, auf welchen Maßstabsebenen wichtige Standortfaktoren der Kreativwirtschaft und Wissensökonomie hauptsächlich räumlich wirksam werden. Die dargestellten Standortfaktoren sollen wesentliche Aspekte schlaglichtartig erfassen, ihre grafische Überlappung deutet an, wie sich Kreativwirtschaft und Wissensökonomie gegenseitig bedingen und ergänzen. Die Abbildung versteht sich als Skizze ohne Anspruch auf Vollständigkeit. Dennoch hilft sie, wesentliche Eigenschaften des systemischen $\mathrm{Zu}$ sammenspiels zu erkennen.

Standortentscheidungen von wissensintensiven Unternehmen werden wesentlich geprägt durch die Nähe zu Kunden und Partnern; Faceto-Face Kontakte sind für die Weitergabe von implizitem Wissen und das Schaffen von Innovationen unabdingbar. Die sich herausbildenden Metropolregionen können als gemeinsamer Nenner der Standortanforderungen der verschiedenen wissensintensiven Branchen und Unternehmenstypen begriffen werden. Sie bieten die erforderliche kritische Masse sozialer Interaktionen und stellen internationale wie innerregionale Erreichbarkeit sicher (Thierstein/Goebel/Lüthi 2007). Innerhalb von Metropolregionen finden sich ausdifferenzierte Lagequalitäten ent- 
Abbildung 2: Skizze zu einem systemischen Zusammenspiel der Standortfaktoren von Kreativwirtschaft und Wissensökonomie nach Raumdimensionen und Maßstabsebenen

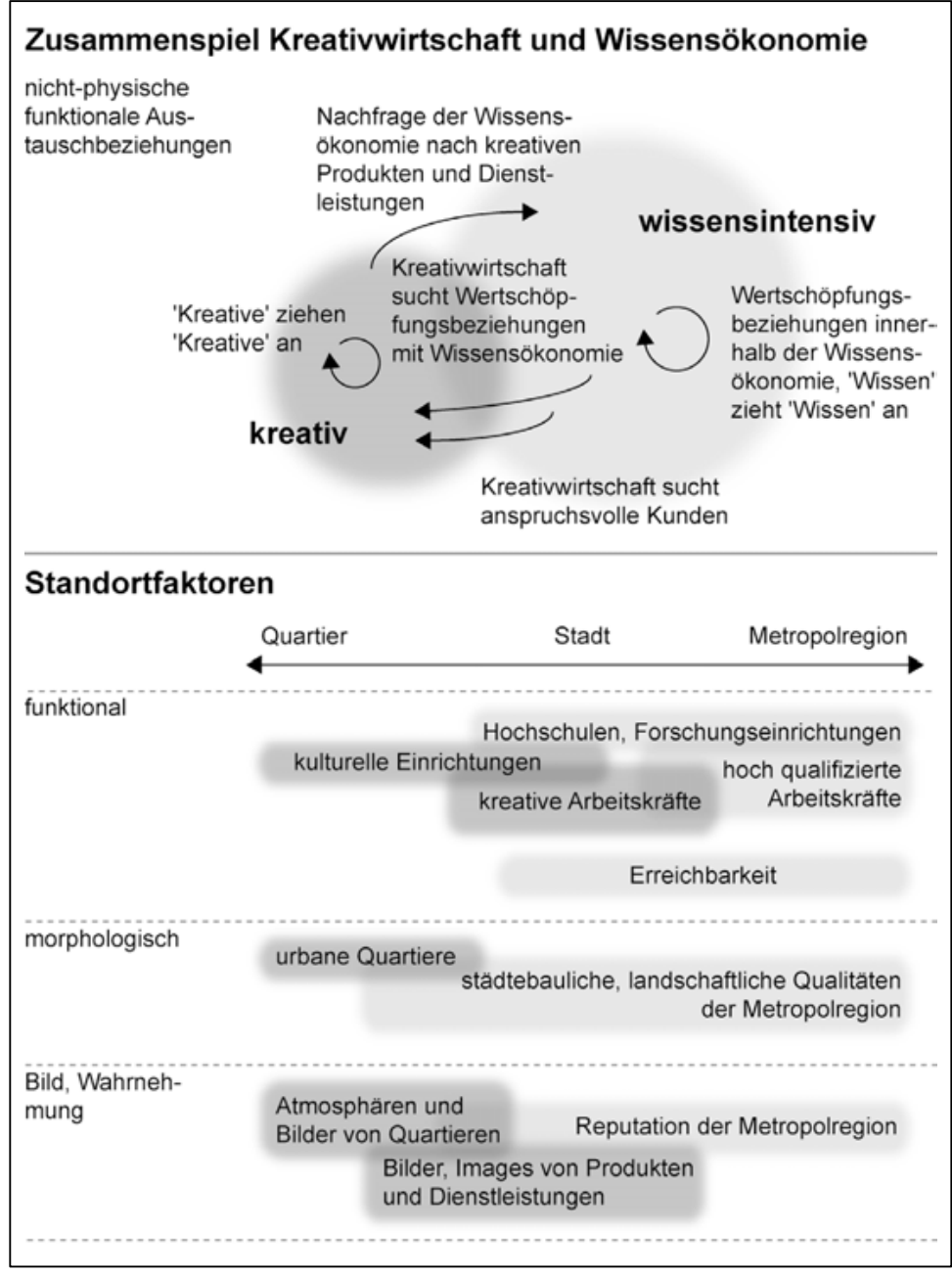

Quelle: eigene Darstellung

sprechend der branchen- und unternehmensspezifischen Anforderungen der Wissensökonomie. Wissensintensive Wirtschaftszweige sind wesentlich angewiesen auf die Verfügbarkeit hoch qualifizierter Arbeitskräfte. Für diese Menschen sind Kultur und Kreativität wichtige Faktoren bei der Wahl des Arbeits- und Wohnorts. Diese alleine reichen aber nicht aus, denn Kreativwirtschaft wird im Bündel mit weiteren 
Qualitäten eines Standorts nachgefragt: Verfügbarkeit von Wissen, Wirtschaftskraft, attraktiver Arbeitsmarkt und internationale Erreichbarkeit (Thierstein/Förster 2008).

Die Standortfaktoren, welche die Kreativwirtschaft direkt nachfragt oder produziert, also anbietet, sind tendenziell räumlich enger gefasst, zum Beispiel auf der Ebene von Quartieren. Durch die Beziehungen zu Kunden und Geschäftpartnern der Wissensökonomie kommen wiederum deren relational begriffenen Standortanforderungen ins Spiel. Die Akteure der Kreativwirtschaft mit ihren Standortanforderungen können daher nicht als Punktgröße verstanden werden. Angebot und Nachfrage von Standortqualitäten der Wissensökonomie und Kreativwirtschaft greifen auf verschiedenen Maßstabsebenen ineinander, Metropolregionen können als ihre räumlich-strategischen Schnittstellen begriffen werden.

\section{Konzepte für ein systemisches Steuern}

Kreativwirtschaft ist ein mit Hoffnungen beladener Gegenstand politisch-planerischer Interventionen - handhabbare, steuerbare Einheiten lassen sich aber nur schwer eingrenzen. Aus der bisherigen Analyse muss folgen, dass das Wohl und Weh der Kreativwirtschaft nur über einen systemischen Ansatz zu steuern ist. Einfache Ursache-WirkungBeziehungen sind nicht erkennbar. Hier bedarf es ausführlicher Systemanalysen. Da wir diese hier nicht leisten können, möchten wir einen Umweg nehmen und abermals indikativ vorgehen.

Ein klar umrissener Gegenstand, welcher von außen gesteuert werden kann, ist nicht auszumachen. Es ist zu erwarten, dass eine Systemanalyse zeigt, dass nur wenige Variablen einer direkten Steuerung überhaupt zugänglich sind und eine aktive Position im System haben. Vor dem Hintergrund dieser Überlegungen halten wir zwei Konzepte für geeignet, um konkrete Maßnahmen zum Steuern des Systems zu entwickeln. Erstens geht es um die Akteursfrage und zweitens um den geeigneten Mix aus Eingriffen.

Ausgehend von dem systemischen Verständnis des Zusammenhangs zwischen Kreativwirtschaft, Wissensökonomie und Metropolregionen, sind Formen der Steuerung zu suchen, die den Steuermann oder viel mehr die Steuermänner als Teil des Systems begreifen. Diejenigen Akteure, die das System selbst zum Laufen bringen sind auch die Akteure, die steuernd eingreifen können. Dieser Gedanke soll im Folgenden am Beispiel des Triple Helix Ansatzes erläutert werden. Das Konzept von regionalen Innovationssystemen basiert auf dem Hauptgedanken, dass 
Innovationen sowohl innerhalb von Unternehmen als auch an der Schnittstelle von Universitäten, Wirtschaftsunternehmen und öffentlicher Verwaltung stattfinden. Der Triple Helix Ansatz (Leydesdorff/ Fritsch 2006; Leydesdorff 2000) begreift Innovation und Kreativität als einen gemeinschaftlichen Prozess, der auf lange Sicht Privatfirmen, Öffentliche Hand und Wissenschaft zusammenführt und damit die Grundlage stärkt für eine gedeihliche Entwicklung von Regionen. Die Innovation Society ist ein Netzwerk europäischer Regionen, darunter die Region Stockholm, die Provinz Nordbrabant und die Metropolregion München, welche zum Ziel hat, Innovationen in den teilnehmenden Regionen zu stärken und die regionalen Innovationspolitiken zu verbessern. Basierend auf dem Gedanken der Triple Helix tauschen öffentliche Verwaltung, Universitäten und Forschungsinstitute sowie Wirtschaftsunternehmen der Partnerregionen ihre Erfahrungen auf Konferenzen aus. Anhand der Ergebnisse des Workshops zu dem Thema „Attracting Talents“ auf der Konferenz der Innovation Society im Mai 2008 in München, wollen wir im Folgenden aufzeigen, wie sich die Kompetenzfelder der drei Triple Helix Partner in Stadtregionen verschränken und auf welchen räumlichen Maßstabsebenen Handlungsmöglichkeiten zu erkennen sind (Thierstein/Förster 2008).

Abbildung 3 zeigt auf der linken Seite hierarchisch von oben nach unten die Standortfaktoren, welche für die Attraktivität einer Stadtregion für hoch qualifizierte und international mobile Talente als besonders bedeutend eingestuft werden. Grundlage bildet eine Umfrage unter den am Workshop teilnehmenden Experten der verschiedenen kooperierenden Stadtregionen.

Mit den Themen „Verfügbarkeit von Wissen“, „landschaftliche Qualität der Region“, „Arbeitsmarkt“, „Kultur“, „Internationalität und Toleranz“, „Erreichbarkeit und Verkehrsinfrastruktur“ sowie „urbane Attraktivität“" zeigen sich Überschneidungen mit den Standortfaktoren des Systems Kreativwirtschaft und Wissensökonomie in Metropolregionen (siehe Abbildung 2).

In der mittleren bis rechten Bildhälfte bewerten die Workshopteilnehmer, welche Partner der Triple Helix diese Standortqualitäten in ihrer Stadtregion produzieren. In der Summe ergänzen und durchdringen sich die Kompetenzfelder deutlich. Viele Standortfaktoren können nur in Kooperation von zwei oder drei Partnern hervorgebracht und bereitgestellt werden. Der Zugriff der öffentlichen Hand auf wesentliche Themen wie Wissen, Wirtschaftskraft und Arbeitsmarkt wird als sehr beschränkt wahrgenommen. 
Es zeigt sich somit deutlich, wie stark die Triple Helix Partner wechselseitig aufeinander angewiesen sind, wenn es um die internationale Attraktivität ihrer Stadtregion im Wettbewerb um Talente geht.

Abbildung 3: Bewertung der Standortfaktoren für die Attraktivität einer Stadtregion für hoch qualifizierte und international mobile Talente nach der Zuständigkeit der Triple Helix Partner

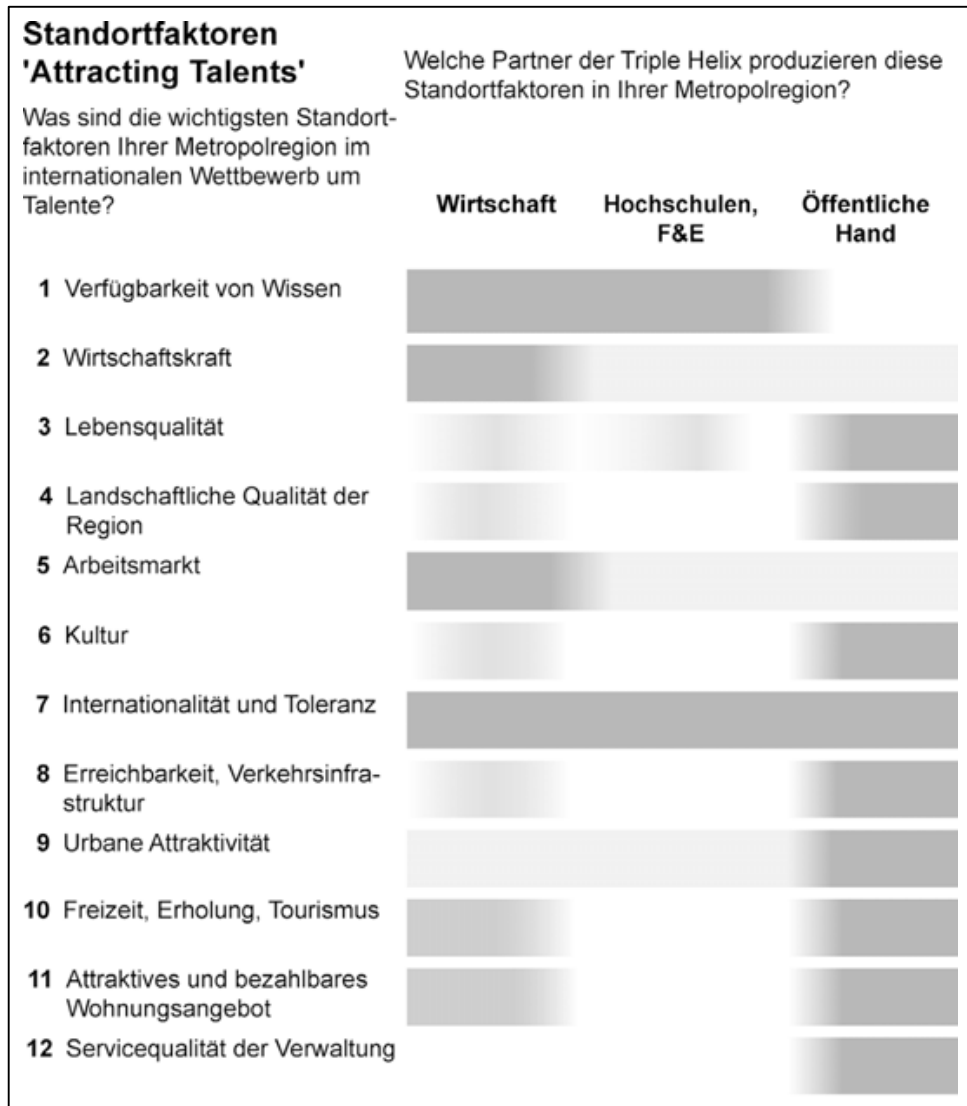

Quelle: eigene Darstellung basierend auf Thierstein/Förster 2008

Gefragt nach den Handlungsfeldern der öffentlichen Hand, welche geeignet sind die Position der eigenen Stadtregion im internationalen Standortwettbewerb um Talente zu stärken, zeigen sich ebenfalls Themen, welche als relevant auch im Zusammenhang von Kreativwirtschaft und Wissensökonomie in Metropolregionen einzuschätzen sind: „öffentlicher Nahverkehr“, „Unterstützung kultureller Aktivitäten“, „Aufwer- 
tung öffentlicher Räume“, „städtebauliche Qualitäten“ und „kulturelles Erbe“ (Abbildung 4). Die Workshopteilnehmer waren aufgefordert zu beurteilen, auf welchen räumlichen Handlungsebenen diese Themen heute von der öffentlichen Hand am wirksamsten angegangen werden können. Obwohl es um Standortqualitäten der Stadtregion im internationalen Wettbewerb geht, wird die größte Hebelwirkung öffentlicher Maßnahmen auf kommunaler Ebene angenommen. Nur bei wenigen Themen wird bereits heute die begrenzte Wirksamkeit der Planungshoheit der Städte und Gemeinden anerkannt. Die Handlungsfelder „öffentlicher Nahverkehr“ sowie, wenn auch etwas schwächer ausgeprägt, ,kulturelles Erbe“ und „Umweltschutz“ verweisen auf die Handlungsebene der Metropolregion. In den Themen „Aufwertung öffentlicher Räume“ und „Schulen und Kinderbetreuung“ werden wichtige Handlungsfelder auf Quartiersebene gesehen.

Überträgt man die Workshopergebnisse, so approximativ sie auch zu verstehen sind, auf das systemische Zusammenspiel von Kreativwirtschaft, Wissensökonomie und Metropolregionen, zeigt sich, worum es im Kern gehen muss: „Multi-level Governance“. Steuerungsmöglichkeiten sind gleichzeitig in verschiedenen Themenfeldern, auf verschiedenen räumlichen Maßstabsebenen, und bei Akteuren unterschiedlicher Welten im Sinne des Triple Helix Ansatzes zu suchen. Vor dem Hintergrund des nur beschränkten Zugriffs öffentlicher Akteure auf diese drei Faktoren scheint die Steuerung wirkungsvoller zu sein, wenn sie auf die Befähigung der Adressaten - Enabling - statt auf direkte Intervention ausgerichtet ist. Das entspricht auch dem systemischen Verständnis von Steuerung, welches den Steuermann selbst als Teil des Systems erkennt.

Aufbauend auf dem bisherigen Erkenntnisstand, werden wir im Schlussteil dieses Artikels eine mögliche Governance-Strategie für das Zusammenspiel von Kreativwirtschaft und Wissensökonomie in Metropolregionen skizzieren. Eine systemische Steuerung kann weder Komplettlösungen, sei es im Sinne eines flächendeckenden räumlichen Vorgehens oder neuer institutionelle Verfasstheiten, bezwecken, noch sich begnügen mit dem Aneinanderreihen von Einzelmaßnahmen. Ziel so-genannter „Wertschöpfungsgeschichten“ ist es, Synergien zwischen funktionalen und räumlichen Komplementaritäten in Metropolregionen auszubauen und zu unterstützen (Thierstein/Goebel/Förster 2006). Am Beispiel der Europäischen Metropolregion München möchten wir diese Strategie näher erläutern.

Standortqualitäten innerhalb einer Metropolregion wie München sind nicht gleichmäßig verteilt. Metropolregionen sind nicht homogen, über die Unternehmensverflechtungen innerhalb der Metropolregion 
Abbildung 4: Beurteilung der vorgeschlagenen Maßnahmen zur Verbesserung der Position der Stadtregion im internationalen Standortwettbewerb um Talente

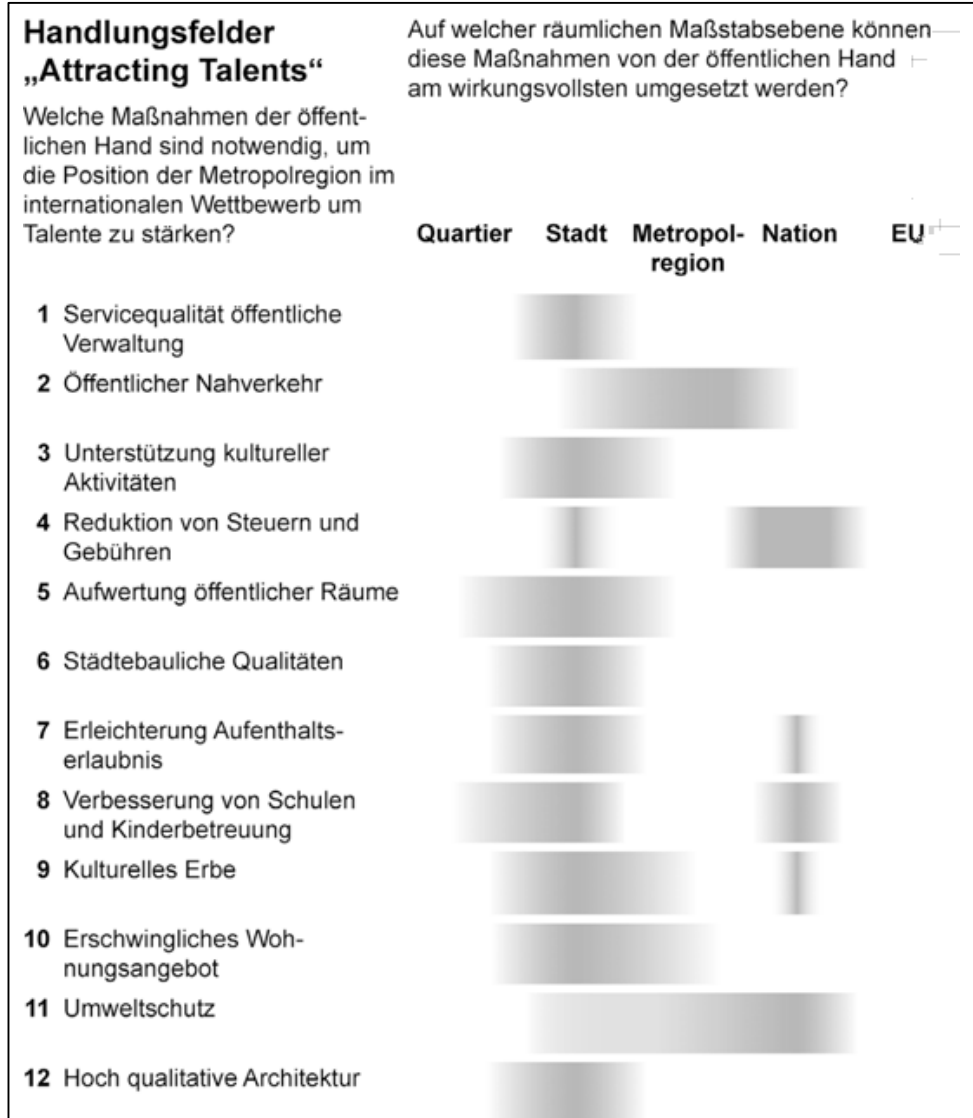

Quelle: eigene Darstellung basierend auf Thierstein/Förster 2008

stellt sich vielmehr eine funktionale Arbeitsteilung zwischen verschiedenen Standorten ein. So tragen die verschiedenen Teilräume der Metropolregion - hoch verdichtete urbane Kerne, Stadtlandschaften im Umland, mittlere und kleinere Zentren, Flughäfen, ländlich geprägte Zwischenräume - weniger über den Wettbewerb untereinander, sondern vielmehr über ihre gegenseitige Ergänzung, also Komplementarität, zu den spezifischen Qualitäten der Metropolregion bei.

Genau diese funktionalen und räumlichen Komplementaritäten sollen mit Hilfe von „Wertschöpfungsgeschichten“ nutzbar gemacht werden. Funktionale Komplementaritäten beziehen sich vor allem auf die 
unternehmerische Logik von miteinander in einer Wertschöpfungskette verbundenen Bausteinen der Produktion von Gütern und Dienstleistungen. Solche Wertschöpfungsketten gibt es innerhalb von Unternehmen oder auch zwischen verschiedenen Unternehmen auch verschiedener Branchen. Innerhalb einer Metropolregion lassen sich solche funktionalen Wertschöpfungsketten räumlich verorten. Verschiedene Teilräume haben dabei spezifische Funktionen, die mit bestimmten Lagequalitäten, also territorialen räumlichen Eigenschaften zusammen hängen. Dadurch entsteht ein räumliches Wertschöpfungssystem, in dem sich funktionales, unternehmerisches Denken und Handeln verbindet mit territorialem, hoheitlichem Denken und Handeln (Abbildung 5).

Der Begriff „Geschichte“ verweist auf die Absicht, eine Vielzahl einzelner Maßnahmen in einen großen, systemischen Zusammenhang zu stellen und diesen auch durch eine bildhafte, erzählerische Komponente zu vermitteln. Die Verknüpfung verschiedener geographischer Maßstäbe und Raumdimensionen, wie sie für funktional verstandene Metropolregionen gilt, spiegelt sich in den Wertschöpfungsgeschichten wider.

Vor dem Hintergrund der räumlichen und thematischen Selektivität sowie des nicht-physischen funktionalen Ansatzes dieser GovernanceStrategie, sind es „Geschichten“, welche die Teile mit einem inneren Faden zusammenhalten. So verbindet die Wertschöpfungsgeschichte „Gesundheit-Wellness-Tourismus“ in der Europäischen Metropolregion München Standorte der Medizin, Forschung und Produktion, vielfach konzentriert im Zentrum der Metropolregion, mit attraktiven Kulturstädten und Erholungslandschaften im Süden und Osten der Metropolregion (Abbildung 5).

Der in Abbildung 6 dargestellte „Umgriff“ dieser Wertschöpfungsgeschichte ist als eine erste räumliche Eingrenzung der Geschichte zu begreifen, in dem die genannten Komplementaritäten zu suchen und knüpfen sind. Das entstehende Gesamtbild setzt auf vorhandenen lokalen und regionalen Wahrnehmungen der Metropolregion auf und verbindet sie auf metropolitaner Ebene zu einer neuen Qualität. 
Abbildung 5: Vorschlag für eine Wertschöpfungsgeschichte „Gesundheit-Wellness-Tourismus" der Europäischen Metropolregion München

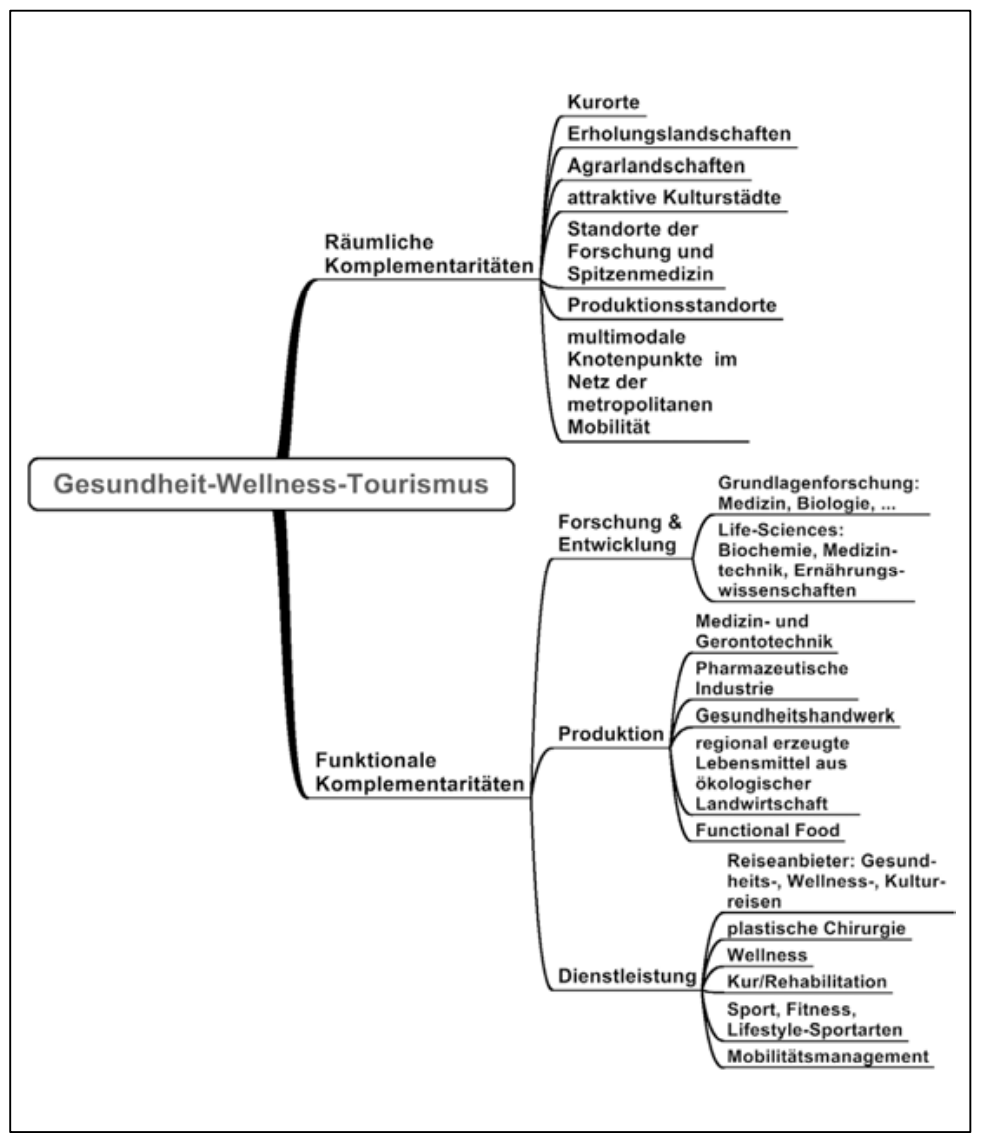

Quelle: Thierstein/Goebel/Förster 2006 
Abbildung 6: Skizze des räumlichen Ineinandergreifens möglicher Wertschöpfungsgeschichten in der Europäischen Metropolregion München

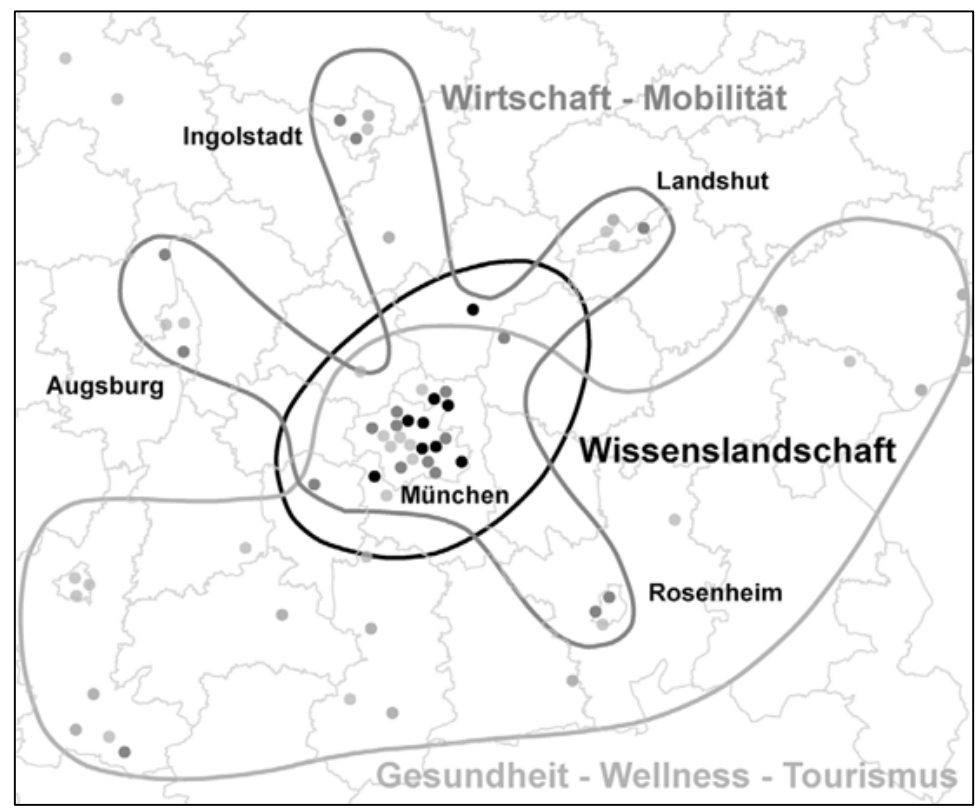

Quelle: Eigene Darstellung basierend auf Förster/Thierstein 2007

Versuchen wir den Transfer dieser Überlegungen auf das Zusammenspiel von Kreativwirtschaft und Wissensökonomie: Wertschöpfungsgeschichten könnten genutzt werden für ein gegenseitiges In-Wert-Setzen von Kreativwirtschaft und Wissensökonomie in Metropolregionen. Funktional gilt es, die wechselseitigen Austauschbeziehungen der beiden Bereiche im Sinne von Wertschöpfungsbeziehungen zu stärken.

Räumlich geht es darum, Synergien durch das Ineinandergreifen der Standortfaktoren von Kreativwirtschaft und Wissensökonomie in Metropolregionen $\mathrm{zu}$ generieren: von der funktionalen Ausstattung mit Wissen, Arbeitskräften und Erreichbarkeit über die morphologischen, also städtebaulichen und landschaftlichen, Strukturen und Qualitäten bis zu den realen, medialen und mentalen Bildern von Metropolregionen.

Begreift man Metropolregionen als einen Prozess, ein im Entstehen begriffenes räumliches Phänomen, welcher maßgeblich durch die wachsende Bedeutung der Wissensökonomie angetrieben wird, kann der Kreativwirtschaft durchaus eine Bedeutung für die Genese und Attraktivität von Metropolregionen attestiert werden. Kreativwirtschaft ist sicherlich kein alleine wirksamer Hebel; sie kann aber die Ausformulie- 
rung bestimmter räumlicher wie funktionaler Qualitäten des Standorts

Metropolregion befördern und helfen, diese bildhaft erzählerisch $\mathrm{zu}$ vermitteln.

\section{Literatur}

Adam, Brigitte/Göddecke-Stellmann, Jürgen (2002): „Metropolregionen. Konzepte, Definitionen und Herausforderungen“. In: Informationen zur Raumentwicklung, Vol. 9, S. 513-525.

Aemisegger, Nina (2007): Die Vernetzung der Kreativwirtschaft Zürich. Eine empirische Untersuchung der privatwirtschaftlichen Kreativbetriebe des Kantons Zürich. Diplomarbeit Geographisches Institut, Abteilung Wirtschaftsgeographie. Zürich: Universität Zürich. www.creativezurich.ch (Zugriff am 31.07.2008).

Blotevogel, Hans H./Schmitt, Peter (2006): „European Metropolitan Regions as a New Discursive Frame in Strategic Spatial Planning and Policies in Germany“. In: Die Erde 137 (1/2), S. 55-74.

Blotevogel, Hans Heinrich (2002): „Deutsche Metropolregionen in der Vernetzung“. In: Informationen zur Raumentwicklung 6/7, S. 345351.

Bonneville, Marc (1994): „Internationalization of Non-Capital Cities in Europe: Aspects, Processes, and Prospects“. In: European Planning Studies 2 (3), S. 267-285.

Budd, Leslie/Amer K. Hirmis (2004): „Conceptual Framework for Regional Competitiveness“. In: Regional Studies 38 (9), S. 1015-1028.

Bundestag (1998): Raumordnungsgesetz (ROG), Berlin: Bundesministerium für Justiz.

Carrincazeaux, Christophe/Lung, Yannick/Vicente, Jérôme (2008): „The Scientific Trajectory of the French School of Proximity: Interactionand Institution-based Approaches to Regional Innovation Systems“. European Planning Studies 16 (5), S. 617-628.

Castells, Manuel (1996): The Information Age: Economy, Society and Culture, Band 1: The Rise of the Network Society, Malden, MA: Blackwell.

Caves, Richard E. (2000): Creative Industries: Contracts between Art and Commerce, Cambridge: Harvard University Press.

Florida, Richard (2002): The Rise of the Creative Class, New York: Basic Books.

Förster, Agnes/Thierstein, Alain (2007): Das Bild der Europäischen Metropolregion München. Protokoll zum Stakeholder-Workshop am 3.7.2007, München: Lehrstuhl für Raumentwicklung. 
Förster, Agnes/Thierstein, Alain (2008): „Calling for Pictures. The Need for Getting a Picture of Mega-City Regions“. In: Alain Thierstein/Agnes Förster (Hg.), The Image and the Region - Making Mega-City Regions Visible! Baden: Lars Müller Publishers, S. 9-34.

Friedmann, John (1986): „The World City Hypothesis“. In Development and Change, Vol. 17, S. 69-83.

Gilly, Jean-Pierre/Torre, André (2000): Dynamiques de proximité, Paris: L'Harmattan.

Grabow, Busso/Henckel, Dietrich/Hollbach-Grömig, Beate (1995): Weiche Standortfaktoren, Stuttgart: W. Kohlhammer.

Hall, Peter/Pain, Kathy (2006a): From Metropolis to Polypolis. In: Peter Hall/Pain, Kathy (Hg.): The Polycentric Metropolis. Learning from Mega-City Regions in Europe, London: Earthscan, S. 3-16.

Hall, Peter/Pain, Kathy (2006b): The Polycentric Metropolis. Learning from Mega-City Regions in Europe, London: Earthscan.

Helbrecht, Ilse (2005): „Geographisches Kapital - das Fundament der kreativen Metropolis“. In: Kujath, Hans Joachim (Hg.): Knoten im Netz. Zur neunen Rolle der Metropolregionen in der Dienstleistungswirtschaft und Wissensökonomie, Münster: LIT Verlag.

Held, Thom/Kruse, Christian (2005): Kreativwirtschaft Zürich. Studie II - Raum für das Kreative. Konzeptionelle Ansätze für den Aufbau eines Clusters Kreativwirtschaft Zürich, Zürich: Wirtschaftsförderungen der Stadt und des Kantons Zürich. www.kulturwirtschaft.ch, Zugriff am 31.7.2008.

Held, Thomas/Kruse, Christian/Söndermann, Michael/Weckerle, Christoph (2005): Kreativwirtschaft Zürich, Synthesebericht. Zürich: Museum für Gestaltung.

Kitson, Michael/Martin, Ron/Tyler, Peter (2004): „Regional Competitiveness: An Elusive yet Key Concept?“'In: Regional Studies 38 (9), S. 991-999.

Kujath, Hans-Joachim (2002): „Die Logiken internationaler und nationaler ökonomischer und politischer Verflechtungen von Metropolregionen“. In: Informationen zur Raumentwicklung 7, S. 431-438.

Legler, Harald/Frietsch, Rainer (2006): Neuabgrenzung der Wissenswirtschaft - forschungsintensive Industrien und wissensintensive Dienstleistungen (NIW/ISI-Listen 2006), Hannover, Karlsruhe: Niedersächsisches Institut für Wirtschaftsforschung, Fraunhofer-Institut für System- und Innovationsforschung.

Leydesdorff, Loet (2000): „The Triple Helix: An Evolutionary Model of Innovations“. In: Research Policy 29, S. 243-255. 
Leydesdorff, Loet/Fritsch, Michael (2006): „Measuring the Knowledge Base of Regional Innovation Systems in Germany in Terms of a Triple Helix Dynamics“. In: Research Policy 35 (10), S. 15.

Michaels, Ed/Handfield-Jones, Helen/Axelrod, Beth (2001): The War for Talent, Boston, MA: Harvard Business School Press.

MKRO (2006): Leitbilder und Handlungsstrategien für die Raumentwicklungspolitik in Deutschland, Bonn: Ministerkonferenz für Raumordnung.

MKRO (1995): Raumordnungspolitischer Handlungsrahmen. Bonn: Ministerkonferenz für Raumordnung.

Oinas, Päivi (Hg.) (2000): Distance and Learning: Does Proximity Matter? Knowledge, Innovation and Economic Growth, Cheltenham: Edward Elgar.

Polanyi, Michael (1966): The Tacit Dimension, New York: Doubleday.

Porter, Michael E. (1990): The Competitive Advantage of Nations, New York: The Free Press.

Referat für Arbeit und Wirtschaft (2007): München - Standortfaktor Kreativität. Studie des Departements für Geographie, LMU München, München: Landeshauptstadt München.

Sassen, Saskia (2001): The Global City: New York, London, Tokyo, 2. Aufl., Princeton/New York: Princeton University Press.

Söndermann, Michael/Weckerle, Christoph/Klaus, Philipp/Bentz, Dominic/Hofstetter, Claudia (2008): Zweiter Zürcher Kreativwirtschaftsbericht. Empirisches Portrait der Kreativwirtschaft Zürich. Geographie der Kreativwirtschaft, Zürich: Wirtschaftsförderung der Stadt Zürich, Standortförderung des Kantons Zürich. www. statistik.zh.ch (Zugriff am 31.7.2008).

Taylor, Peter J. (2004): World City Network: A Global Urban Analysis, London: Routledge.

Thierstein, Alain (1996): Auf der Suche nach der regionalen Wettbewerbsfähigkeit - Faktoren und Einflussmöglichkeiten. In: Raumforschung und Raumordnung 54 (2/3), S. 193-202.

Thierstein, Alain/Förster, Agnes (2008): Working Group A. Strategies of Knowledge Regions - Attracting Talents and Mobilizing Competences. 3rd Innovation Society Conference 29.-30.5.2008, Munich. www.innovationsociety2008.de (Zugriff am 31.7.2008).

Thierstein, Alain/Goebel, Viktor/Förster, Agnes (2006): Das Feuer in der Europäischen Metropolregion München entfachen. Expertise zum Aufbau eines Initiativkreises Europäische Metropolregion München. München: Landshauptstadt München.

Thierstein, Alain/Goebel, Viktor/Lüthi, Stefan (2007): Standortverflechtungen der Metropolregion München. Über Konnektivität in der 
Wissensökonomie. Endbericht Forschungsprojekt. München: Lehrstuhl für Raumentwicklung, TU München. www.raumentwicklungtum.de (Zugriff am 31.7.2008).

Thierstein, Alain/Kruse, Christian/Glanzmann, Lars/Gabi, Simone/ Grillon, Nathalie (2006): Raumentwicklung im Verborgenen. Untersuchungen und Handlungsfelder für die Entwicklung der Metropolregion Nordschweiz, Zürich: NZZ Buchverlag.

Thierstein, Alain/Schedler, Kuno/Bieger, Thomas (Hg.) (2000): Die lernende Region. Regionale Entwicklung durch Bildung, Zürich: Rüegger.

Vester, Freceric (2007): Die Kunst vernetzt zu denken. Ideen und Werkzeuge für einen neuen Umgang mit Komplexität, 6. Auflage, München: Deutscher Taschenbuch Verlag.

Watzlawick, Paul/Beavin, Janet H./Jackson, Don D. (2000): Menschliche Kommunikation. Formen, Störungen, Paradoxien, 10. Auflage, Bern: Hans Huber.

Whitehead, Alfred N. (1988): Wissenschaft und moderne Welt, Frankfurt/Main: Suhrkamp.

Weckerle, Christoph/Söndermann, Michael (2005): Kreativwirtschaft Zürich. Studie I: Der privatwirtschaftliche Teil des kulturellen Sektors im Kanton Zürich. Zürich: Wirtschaftsförderung des Kantons Zürich, Wirtschaftsförderung der Stadt Zürich. www.kulturwirtschaft.ch (Zugriff am 31.7.2008). 



\section{Alltagszustand „Risikobereitschaft“? Ethnografisch-kulturwissenschaftliches Wissen über Risiko in der Kreativwirtschaft}

ALEXA FÄRBER

The culture/creative industries are a high risk sector with extremely variable market chances. This is true for global culture/film productions as well as for local culture/creative scenes or SMEs acting on a regional level.

(Fesel/Söndermann 2007: 25).

Die Studie der UNESO zur Kultur- und Kreativwirtschaft in Deutschland zeichnet ein insgesamt positives Bild dieses Sektors, ist hier doch in den vergangenen Jahren ein Wirtschaftsbereich entstanden, der nicht nur wirtschaftliche Erträge erbringt, sondern auch dort, wo sich Kreative sichtbar ansiedeln, einen Imagegewinn verspricht. Neben dem ökonomischen und symbolischen Kapital, das sich hier akkumulieren lässt, bringt dieser Sektor jedoch auch die genannten strukturellen Unwägbarkeiten mit sich: Wenn Kreativwirtschaft mit "hohem Risiko“ verbunden ist, dann baut sie offenbar auf eine gesteigerte Risikobereitschaft. Die Charakterisierung der „kreativen Klasse“, wie sie in Anlehnung an Richard Florida äußerst populär geworden ist, unterstreicht diese Annahme, indem Risikobereitschaft als einer der gemeinsamen Nenner der vielfältigen ProtagonistInnen darstellt wird (Florida 2002). Risiko und die Bereitschaft, es einzugehen, müssen indes, und das interessiert an dieser Stelle, als hochgradig individualisiert angesehen werden und zwar für ein breites Spektrum an Akteuren. 
Das Forschungsfeld „Kreativwirtschaft“ hat sich in den vergangenen zehn Jahren vor allem als ein multi-disziplinäres herausgebildet, zumal dann, wenn es mit Fragen der Stadtforschung verkoppelt wird: Sei es die in der geschichtswissenschaftlichen Forschung angestoßene Frage nach historischen Formen kreativer Milieus (Heßler/Zimmermann 2008), oder die in Soziologie, Wirtschafts- und Kulturgeografie durchgeführten Untersuchungen zu gegenwärtigen Formen der Clusterbildung (Krätke 2002; Scott 2000) und der Genese spezifischer Stadtentwicklungsparadigmen der „kreativen Stadt“ (O’Connor 2005; Heßler 2007). Ethnografische Studien haben sich zudem der genauen Beobachtung von Milieus gewidmet, von professionellen und subkulturellen kreativen Praktiken und ihrer Materialisierung im Stadtraum (Lange 2007; Schwanhäußer 2008; Vogt 2005).

Die Spezifik ethnografisch arbeitender Kulturwissenschaften liegt in der Generierung von Material durch Teilnehmende Beobachtung: Diese in die Tiefe einzelner Fallbeispiele gehende Perspektive passt sich im Forschungsprozess den Entwicklungen des Forschungsfeldes an, sie ist multi-lokal und geht vergleichend vor. Die Analyse des auf diese Weise selbst generierten qualitativen Materials bezieht quantitative Daten und diskursanalytische Textauswertung ein, überprüft inwiefern das Material zum gewählten disziplinären Theorieansatz beiträgt und unterzieht diesen Erkenntnisprozess einer reflexiven Perspektive (Schmidt-Lauber 2007). Im interdisziplinären Stadtforschungszusammenhang widmet sich eine solche theoretisch informierte und reflexive Mikro-/Tiefenperspektive vor allem der Alltagskultur (Kokot 2000). Für das Verständnis der Bedeutung von Risiko in der Kreativwirtschaft und des Umgangs mit Risiko ist eine solche Perspektive deshalb relevant, weil sie Attraktion und Bürde risikoreicher Praktiken im Alltag nachzeichnen und daraus Wissen über mögliche Formen kollektiver Absicherung bereitstellen kann. Dieser Beitrag stellt nur einen ersten Schritt in diese Richtung dar und hat zum Ziel, die zu untersuchenden Akteursgruppen, Praxisformen und stadtspezifischen Ausformungen von Risiko in der Kreativwirtschaft zu umreißen.

\section{Risiko als Untersuchungsgegenstand}

„Risiko“ zählt sicherlich zu den Schlüsselbegriffen der spätmodernen Gesellschaftsanalyse. Ulrich Beck hat erstmals Mitte der 1980er Jahre und jüngst in seiner deutschen Fassung der ,Weltrisikogesellschaft" (2007) die überindividuellen Dimensionen globaler Risiken in ihrer gesellschaftlichen Ausformung untersucht und zahlreiche Studien in die- 
sem Zusammenhang angestoßen. Für die Soziologie der Risikogesellschaft gilt Risiko als eine Begleiterscheinung nicht etwa des Versagens, sondern des Erfolgs der kapitalistischen Gesellschaftssysteme. Die Risikogesellschaft zeichnet sich nach Beck durch die zunehmende „Bedeutung von Entscheidung, Unsicherheit und Wahrscheinlichkeit“" aus (Beck 2007: 19). Damit sind drei elementare Bestandteile von Risiko benannt, denen eine ethnografisch-kulturwissenschaftliche Perspektive nachgehen sollte, um die kulturellen Formen von Risiko akteurs-, praxis- und stadtorientiert zu untersuchen.

Was die Soziologie der Risikogesellschaft anhand konkreter Ereignisse und ihrer gesellschaftlichen Verarbeitung untersucht, interessiert die Gouvernementalitätsstudien mit Blick auf Subjektkonstruktionen. Ausgehend von den spezifischen, diskursiv vermittelten Anforderungen an spätmoderne Subjekte zeichnen diese Studien eine Risikologik nach, die an sich nicht neu ist, aber ihren Adressatenkreis erheblich erweitert hat: Ein „Modell der Konstruktion und Bearbeitung von Risiken“ greift auf „,nahezu alle gesellschaftlichen Bereiche“ über, so Henning SchmidtSemisch. Dieses Modell hat ,die Eigenverantwortung der Subjekte zu ihrem Ziel und ihrer Voraussetzung zugleich, gelten soll sie im Guten wie im Schlechten. Indem Autonomie und Responsibilisierung zusammenfallen, die Subjekte also stets auch mit der Unsicherheit des selbst verschuldeten Scheiterns leben müssen, schreibt sich die marktwirtschaftliche Risikologik auf ambivalente Art und Weise in Befindlichkeit, Handeln und Denken der Individuen ein“" (Schmidt-Semisch 2004: 223f.).

Dieses marktförmige und subjektivierte Risiko richtet sich an ein „,unternehmerisches Selbst“ und lässt sich in Anlehnung an Klassiker der Wirtschaftswissenschaften in „reine Ungewissheit“ und „kalkulierbare Risiken“ unterscheiden: „Ohne rationale Planung und Kontrolle kommt keine Unternehmung aus, aber zum Unternehmer im Sinne Knights wird nur, wer immer wieder den Schritt hinaus ins Ungewisse wagt.“ (Bröckling 2007: 119)

Es sind die subjektkonstituierenden Anforderungen und Freiheitsversprechen, nicht die sich daran abarbeitenden konkreten und beobachtbaren Praktiken, die in den Gouvernementalitätsstudien im Vordergrund stehen und einer kritischen Analyse unterzogen werden. Kreativität gilt dabei als ein zentraler Imperativ, Risiko als eine Begleiterschei- 
nung. ${ }^{1}$ Dies hat auch die 2003 vom Bundestag als Enquete-Kommission eingesetzte Expertengruppe „Kultur in Deutschland“ erkannt, die Künstler als die „Prototypen neuer Selbst- und Einzelunternehmer“ identifiziert, die ,sich durch Flexibilität, Mobilität, Parallelarbeit und ein hohes $\mathrm{Maß}$ an Risikobereitschaft" auszeichnen (Deutscher Bundestag 2007: 290). Gleichzeitig muss sie aber den geringen Kenntnisstand über die Bedeutung dieses „Prototyps“ für die Alltagspraxis feststellen. Diese „derzeit noch nicht präzise beschreibbare Mischung aus wirtschaftlichen Armutsrisiken und subjektiven Autonomiegewinnen“ (ebd.: 295) bietet aber eine Reihe von Beobachtungspunkten für die ethnografisch-kulturwissenschaftliche Forschung.

\section{Ethnografisch-kulturwissenschaftliche Perspektiven auf Risiko und Kreativwirtschaft}

Den Beitrag ethnografischer Untersuchungen in der ,gegenwärtigen Krise der Arbeitsgesellschaft und des Sozialstaates“ sieht Bernd Jürgen Warneken weniger in der realistischen Darstellung der ökonomischen Situation und Bewältigung neuer Arbeitsformen. Vielmehr sollte sich das ethnografische Interesse auf die weit reichende Ambivalenz einer (vormals im Kreativitätskonzept eingelagerten) ,popular agency“ richten. Es sollte

dazu beitragen, die Innenseite dieses Geschehens zu erhellen. Werden die Bewältigung unterschiedlicher Jobs, die zeitliche Koordination der verschiedenen Arbeitsaufgaben sowie das Herausschlagen von Restzeiten für das Privatleben nur als Stress oder auch als Selbstbestätigung empfunden? Entspricht der Kreativitäts-Zuschreibung von außen ein Stolz auf die eigenen Fähigkeiten und Befriedigung über das Geleistete? [...] Und es wäre sicherlich voreilig, ein solches Selbstverständnis als Illusion abzuhaken, statt nach Realien oder Potentialen zu suchen, die solch eine Selbstsicht erklären und es manchmal sogar rechtfertigen, ihm vorwärtstreibende Momente zuzuerkennen. (Warneken 2006: 125)

Diesem Forschungsauftrag kommt die Arbeitskulturforschung bereits nach, indem sie die realen Seiten der Ambivalenz von Selbstausbeutung und Selbstverwirklichung, von Armutsrisiken und Autonomiegewinnen

1 Die Kritik an Richard Floridas Konzept der kreativen Klasse richtet sich deshalb auch häufig gegen die Normativität, die es aus eben jenen Anforderungen zieht. 
beschreibt und nachvollziehbar macht (Moser 2003; Seifert/Götz/Huber 2007). Diese Forschungsperspektive kann durch Fragen nach Risikopraktiken nochmals zugespitzt werden. Für den spezifischen Bereich der urbanen Kreativwirtschaft und der darin zu beobachtenden alltagskulturellen Bedeutung von Risiko wären dann drei Untersuchungsperspektiven $\mathrm{zu}$ unterscheiden: eine akteurszentrierte, eine praxiszentrierte und eine auf die Stadtform konzentrierte Perspektive. Im Zusammenspiel dieser Ansätze können die involvierten Akteurskonstellationen, Risikopraktiken und stadtspezifischen Ausformungen von Risikobereitschaft erhoben und auf ihre Alltagsrelevanz hin untersucht werden.

\section{Akteurszentrierte Perspektive}

In einer akteurszentrierten Perspektive ist zunächst einmal von Interesse, für wen welche Risiken innerhalb des Kontexts der Kreativwirtschaft gelten? Denn die Komplexität - und die von der UNESCO attestierte große Spannbreite an Erfolgs- oder Misserfolgschancen - geht u. a. auf die historisch verankerte Vielfalt involvierter Akteursgruppen zurück. Wie lassen sich Akteursgruppen beschreiben und wie ist Risiko und Risikobereitschaft zwischen ihnen verteilt?

Die ethnografische Studie der Stadtsoziologin Silke Steets über Leipzigs Kreativwirtschaft liefert für die Beantwortung dieser Fragen eine Reihe von Ansatzpunkten (Steets 2008). ${ }^{2}$ Leipzig scheint vor wie nach der Wende eine für kreative Milieus (Metzler 2008: 42f.) ${ }^{3}$ attraktive Stadt gewesen und noch heute zu sein: Als Knotenpunkt für Netzwerke von Kreativen kann die mit der Leipziger Hochschule für Grafik und Buchkunst einher gehende Kunstszene, vor allem im Bereich Malerei, gelten, die seit Ende der 1990er Jahre über den bundesdeutschen

2 Steets rekonstruiert in dieser Ethnografie die (Vor-)Geschichte der Kreativwirtschaft in Leipzig ab den frühen 1990er Jahren über ca. 15 Jahre.

3 Mit dem Begriff der „kreativen Milieus“ untersucht die Historikerin Birgit Metzler am Beispiel des Nauwieser Viertels in Saarbrücken die Geschichte kultureller Netzwerke ab den 1970er Jahren bis heute. Interessant an diesem Fall ist die weit über das Viertel und die Stadt hinausreichende Sichtbarkeit des an politischen Zielen orientierten alternativen „kreativen Milieus", das sich aber weder nachhaltig als Zentrum von kreativwirtschaftlichen Aktivitäten etablieren konnte noch in das Bild der Stadt als solche hätte vordringen können. Metzger verweist damit auf die Diskontinuitäten kreativer Milieus und, dass ,[t] he simple presence of a bohemian, alternative milieu in a city seems not to be a sufficient condition for economic development as claimed especially by Richard Florida“. Um tiefere Einsicht über die Bedingungen von Erfolg zu erlangen, müssten deshalb auch Fälle von Misserfolg in der Kreativwirtschaft untersucht werden. 
Kontext hinaus zur Verknüpfung der Stadt mit dem globalen Kunstmarkt geführt hat (Steets 2008: 171ff.). Während dieser Bereich der Kreativwirtschaft, nicht nur in Leipzig, zu den schillerndsten zählt, was sich u. a. in der besonders großen Spannbreite der Marktchancen für die Individuen ausdrückt, sind in den frühen 1990er Jahren subkulturelle, spartenübergreifende kulturelle Netzwerke entstanden. $\mathrm{Zu}$ dieser Zeit bildeten sich diese Netzwerke bereits zu einem großen Teil aus Ost- wie Westdeutschen, denn viele der jungen AkteurInnen waren aus den alten Bundesländern zugezogen. Eine spezifische Akteurskonstellation, die der historischen Umbruchssituation geschuldet war und derjenigen im Ostteil Berlins ähnelte. Diese Spezifik beruhte auch auf der besonderen räumlichen Situation, d. h. neben den industriellen Brachen auf dem Leerstand im Wohn- und Gewerberaumbereich. Steets vollzieht in ihrer Studie „Wir sind die Stadt!“ das Werden dieser Netzwerke nach, die sich zunehmend professionalisieren und Leipzig nur zum Teil explizit auch als Identifikationsraum benennen.

Für einen der zentralen Akteure eines kulturellen Netzwerkes „komme es vor allem auf das Netzwerk an Leuten an, mit denen er Projekte realisieren kann. Und das sei in Leipzig ohne Zweifel vorhanden, aber nicht direkt an die Stadt oder den lokalspezifischen Kontext gebunden“ (Steets 2008: 185). Dass er hier dennoch schon seit zehn Jahren kontinuierlich lebe, interpretiert Steets nicht etwa mit dem Konzept der „sticky places“, die deshalb attraktiv sind, weil an ihnen ein gelungenes „Zusammenspiel zwischen Wissensmilieus [...] und lokalen Organisationen beziehungsweise institutionellen Strukturen" stattfinde (ebd.: 189). Vielmehr zeichneten sich für diese Akteure die von ihnen hergestellten/genutzten Orte durch die Vorstellung eines „nutzungsoffenen Raums [aus], eines Raums der kreative Formen der Aneignung zulässt, an dem man die ,richtigen Leute" trifft und der neuartige Formen der Kulturproduktion erlaubt“ (ebd.: 185). Deshalb schlägt Steets den Begriff der ,wohnzimmertypischen Vergemeinschaftungsform“ vor.

Hinsichtlich der Verteilung von Risiko und Risikobereitschaft liefert die Studie einige Ansatzpunkte: So verweist die von den AkteurInnen hervorgehobene geringe Anzahl von Orten, an denen sich in der Entstehungsphase immer die selben Personen getroffen hätten, zum einen auf den Pionierstatus, den sie sich rückblickend zuschreiben - und damit auch auf ihre Risikobereitschaft. Andererseits relativiert genau diese Übersichtlichkeit den Grad des Risikos für kreative Unternehmungen: die neue Situation wird dadurch in ihren Unwägbarkeiten überschaubar und kalkulierbar. Wer agiert also innerhalb dieses Feldes mit hoher Risikobereitschaft, wer mit weniger oder gar ohne? Dabei gilt es in diesem Fall zu bedenken, dass diese Situation zwar für alle gleichermaßen neu 
war. Sie war aber für Ost- und Westdeutsche unterschiedlich herbeigeführt worden (Systemwandel vor Ort vs. Systemtransfer und Ortswechsel usw.) und man ist ihr deshalb mit einem unterschiedlichen Repertoire an Alltagspraktiken begegnet; Risikobereitschaft und der Umgang mit Risiko müssen davon nachhaltig geprägt worden sein.

Die Studie zu Leipzig beschränkt sich mit Blick auf die Akteurskonstellationen nicht auf die Untersuchung der Kreativen oder UnternehmerInnen selbst. Sie bezieht die an der politischen und wirtschaftlichen Identifizierung, Steuerung und Aufwertung der Kreativwirtschaft Beteiligten ein, was für alle akteurszentrierten ethnografischen Untersuchungen gelten muss. Dazu zählen auf politischer Ebene Bundesministerien und einzelne kommunale Verwaltungen, zudem öffentliche wie private Institutionen (Handelskammern, Forschungsinstitute); darüber hinaus aber auch herausragende, impulsgebende Einzelpersonen und Medien (Friedrichs 1998). Über welche Instrumente werden hier Interessen und Akteursgruppen konstruiert (bspw. in Form von Kulturwirtschaftsberichten und der Benennung von dazu zählenden Sektoren, Institutionen, Einzelpersonen) und wer trägt welches Risiko innerhalb des damit nachweisbaren Zusammenhangs „Kreativwirtschaft"? Nicht alle Akteursgruppen dürften hier (demselben) Risiko begegnen.

\section{Praxiszentrierte Fragestellungen}

Eine empirisch praxiszentrierte Forschungsperspektive widmet sich nicht der Frage, ob Individuen und Gruppen in unterschiedlichem Maße Risikobereitschaft umsetzen, sondern wie sie dies tun und wie sie mit verschiedenen Formen von Risiko umgehen, die der KreativwirtschaftsKomplex hervorbringt. Wie wird in den einzelnen Akteurskonstellationen welches Risiko verhandelt - was schafft Anreize, was dämpft Risikobereitschaft? In welche Richtung führen Kalkulationspraktiken und -instrumente?

Im Zentrum steht dann das Herausarbeiten konkreter Praktiken, die das Verhältnis von hohem Entscheidungszwang, gesteigerter Unsicherheit und Kalkulation von Wahrscheinlichkeit des Erfolgs oder des Scheiterns bestimmen. Wie werden diese Wahrscheinlichkeiten „kalkuliert“ bzw. praktisch umgesetzt?

Obwohl Risikobereitschaft und damit einhergehende kulturelle Praktiken in der Studie von Steets nicht thematisch ausgeführt werden, zeigt die detaillierte Beschreibung des öffentlich-privaten Charakters dieser szeneförmigen Netzwerke, dass ein individuelles Risiko für eine neue Unternehmung innerhalb des jeweiligen Netzwerkes abgefedert wird. So auch für die Autorin selbst, deren Zugang zum Forschungsfeld über die 
eigene Beteiligung an Kulturproduktionen zustande kam. Diese Form der Teilnehmenden Beobachtung hatte „,den Arbeitsalltag freischaffender Kulturproduzenten und eine sehr persönliche Erfahrung des ökonomischen Überlebenskampfes“ (Steets 2008: 18) zum Gegenstand und erlaubt ein differenziertes Bild der sozialen Praktiken der „Szenevergemeinschaftung“ (ebd.: 211). Diese Praktiken erzeugen eine geringe Fallhöhe für die einzelnen Akteure, indem die Überschaubarkeit der Netzwerke beibehalten wird. Sie äußern sich darin, dass das (zahlende) $\mathrm{Pu}$ blikum einer Kulturproduktion vor allem aus den anderen Szenemitgliedern besteht und dass sie vielfältige, zeitlich begrenzte Kooperationen zwischen den einzelnen Akteuren erzeugen: „Gruppen formieren sich für eine Filmproduktion, eine Bandgründung, eine Clubshow, ein zweiwöchig betriebenes Café, eine künstlerische Intervention oder die Aufnahme eines Hörspiels und lösen sich nach Beendigung des Projektes wieder auf.“ Ausschlaggebend ist: „Als Potentialität aber existieren sie weiter: Man bleibt in Kontakt, behält unter Umständen sogar das gemeinsame Label und wenn sich ein neues Projekt ergibt, kommt man wieder zusammen oder bildet neue Kooperationen.“ (Ebd.: 17).

„Wahrscheinlichkeit“, ein Element von Risiko, wird hier positiv gewendet zur „Wahrscheinlichkeit wieder zu kooperieren“ und nicht etwa als mangelnde Planbarkeit oder Ungewissheit artikuliert. Flankiert wird diese projektförmige Risikopraxis von geringen finanziellen Investitionen und einer Mischfinanzierung des Lebensunterhalts, die aus unterschiedlichen Arbeitsverhältnissen resultiert.

Ebenfalls auf individuellen „Mischfinanzierungen“ beruht das Beispiel einer „Verkaufsplattform“ für Design in Berlin. Im Unterschied zu den bei Steets beschriebenen kulturellen Netzwerken sind die hier angewandten kooperativen Praktiken jedoch nicht spartenübergreifend und führen nicht zu einer Szenevergemeinschaftung; sie dienen allein der Kalkulation von Erfolgs- oder Misserfolgschancen.

Bei dem hier gewählten Beispiel aus einer Langzeituntersuchung zu „Kaufhausprojekten“, die sich auf Design aus Berlin spezialisiert haben, (Färber 2008: 417-425) handelt es sich um ein Kaufhaus, das als Verkaufs- und Arbeitsraum in einem Gebäude im Stadteil Friedrichshain von wechselnden KooperationspartnerInnen betrieben wird. An diesem anfangs noch vom Quartiersmanagement unterstützten Kaufhaus sind ExistenzgründerInnen anhand unterschiedlicher Praktiken beteiligt (zu Beginn noch gefördert durch das Format der Ich-AG). Das geringste finanzielle und zeitlich-räumliche Engagement gehen diejenigen ein, die sich ein Regal mieten, um ihre Waren auszustellen und zum Verkauf anzubieten. Gängistes Model ist jedoch eine kombinierte Werkstatt- und Verkaufsraumnutzung auf Grundlage eines monatlichen Mietbeitrags 
und der Abgabe von $10 \%$ der Einnahmen in die gemeinsame Kasse. Die tägliche Anwesenheit ist in keinem der beiden Modelle verpflichtend, da der Verkauf von einer Person gehandhabt werden kann, die dann die Kassen der jeweils nicht anwesenden DesignerInnen mit verwaltet und einen Abschlag für diese Tätigkeit erhält. Auf diese Weise ist die Ressource Zeit relativ frei verfügbar und wird von einigen der ca. zehn ständigen MieterInnen dahin gehend genutzt, dass sie die eigenen Waren an anderen Orten (z. B. Märkten, weitere Geschäfte oder auch in Onlineshops) anbieten - und überhaupt entwerfen und fertigen. Das finanzielle Risiko, das ein etwaiger geringer Umsatz im Kaufhaus selbst darstellen könnte, wird durch diese Mischformen ausgeglichen, indem vom Kaufhaus aus räumlich flexibel gehandelt wird. Die Fluktuation ist indes relativ groß, von den anfänglichen MieterInnen sind nach vier Jahren nur noch zwei vertreten. Viele sind „Pleite gegangen“, wie die Hauptmieterin ohne Umschweife feststellt - oder haben sich, sobald sich ein gewisser Erfolg abzeichnete, aus der Gemeinschaftsnutzung verabschiedet und ihre eigenen Geschäfte eröffnet.

Insgesamt zeichnet sich das Projekt zum einen durch festgelegte Praktiken aus, die zu einer Minimierung der Wahrscheinlichkeit des Scheiterns beitragen sollen: das Teilen von Raum und Zeit, die anteilige Verteilung von Verantwortung und Ressourcen. Darüber hinaus bleibt aber jeder/m einzelnen überlassen, sein/ihr Geschäft über das Kaufhaus hinaus zu betreiben. Dem unternehmerischen Risiko wird so gesehen temporär gemeinschaftlich und räumlich flexibel begegnet. Die Konzentration auf diese relative Absicherung hängt von den individuellen Ressourcen ab, das jeweilige Mischverhältnis zeichnet sich als Risikobereitschaft ab.

Langfristige Teilnehmende Beobachtung mit der Perspektive „Risiko und Risikobereitschaft" wird genaueren Aufschluss darüber geben, wie sich Planung unter Bedingungen der Planungsunsicherheit vollzieht, wie Absicherung unter Bedingungen der Unsicherheit und berechenbarer Risiken geschieht.

\section{Form- und stadtzentrierte Fragestellungen}

Schließlich stellt sich die Frage, in welcher Form Risiko in der Kreativwirtschaft neben seinen ökonomischen Formaten (von privaten Krediten bis zum Risikokapital) auftritt: Welchen kulturellen Ausdruck finden mangelnde Planbarkeit, (fehlende) ökonomische und soziale Absicherung oder der Gewinn/Verlust an symbolischem Kapital, den die Wahrscheinlichkeit des Erfolgs oder des Scheiterns in der Kreativwirtschaft 
mit sich bringen kann? Wenn „Selbstbestimmung und soziale Deklassierung [...] in der Kreativwirtschaft offenbar untrennbar miteinander verknüpft" sind (Manske 2008: 52; 2007), in welcher Form und in welchem Medium artikuliert sich das Paradox des Risikos im Alltag der AkteurInnen: in Form von Gefahr, Absicherung oder „Risikofreude“, in ironischen Selbstreflexionen, Rationalisierungen, die bspw. ohne digitale Medien nicht zu erlangen wären?

Ohne den Bezug zur akteurs- und praxiszentrierten Perspektive lässt sich die Frage der Form sicher nicht differenziert beantworten. Und auch eine stadtanthropologische Perspektive muss hier zu Rate gezogen werden, die Risikoformate, Praktiken und Akteurskonstellationen hinsichtlich ihrer Einbettung in die „Eigenlogik“ der jeweiligen Stadt erklärt (Berking/Löw 2008).

Risiko als kalkulierbare Möglichkeit des Erfolgs und des Scheiterns hat sich in Berlin als Topos ganz explizit Eingang in das Feld der urbanen Kreativwirtschaft verschafft: in öffentlichen Darstellungen der städtischen Wirtschaftslage und in Role-models. Dass Kulturproduktion in Berlin heute nämlich als florierende Kreativwirtschaft wahrgenommen wird, hat sie auch einer Vielzahl diskursiver Anstrengungen zu verdanken, die Wahrscheinlichkeiten des Erfolgs und des Scheiterns thematisieren und u. a. dadurch kalkulierbar machen.

Eine Analyse der diskursiven Reevaluierung und Einbettung einer als stagnierend wahrgenommenen wirtschaftlichen Gesamtlage des Landes Berlin und einer gleichzeitig dynamischen Kulturproduktion von Seiten öffentlicher und privater Institutionen, wie dem Senat und der Industrie und Handelskammer (IHK), zeigt eines: Alle Akteure, die sich mit der wirtschaftlichen Lage Berlins als Ganzes auseinander setzen müssen, können die Spannung zwischen ökonomischer Ödnis einerseits und florierender Kultur und Kulturproduktion andererseits, nur als Gegensatz darstellen. Gemäß der unterschiedlichen Zuständigkeiten, Positionen und Kontexte wird diese Spannung zwar unterschiedlich aber stets als inkommensurabler Gegensatz formuliert. So schreibt die IHK Berlin in einer Pressemitteilung zur Gesamtlage des industriellen Sektors:

Die Industrie in Berlin ist anders, aber sie ist quicklebendig. Sie ist geschrumpft aber mit ihren Produkten ist sie auf den Weltmärkten wettbewerbsfähig. [...] Die Industrie beschäftigt weniger Mitarbeiter, deren Qualifikation und durchschnittliches Einkommen sind aber höher. ${ }^{4}$ 
Wirtschaftssenator Harald Wolf (Die Linke) stellt als „Grunddilemma, das wir an unserem Standort haben“ fest: „Realität und Potenzial klaffen in Berlin weit auseinander.“ (Waldermann 2005) Bürgermeister Klaus Wowereit (SPD) spricht laut Medienberichten auf einer Dienstreise nach London von ,den maroden Finanzen und wirtschaftlichen Strukturen, die man nicht gerade als gesund bezeichnen könne. Aber Berlin sei eine junge, ,unfertige Stadt", in der man mitmischen könne." Für dieses Dilemma findet er das äußerst medienwirksame Bild: Berlin sei „Arm aber sexy“ (Thibaut 2003). Die Wahrscheinlichkeit ökonomischen Scheiterns tritt in dieser anthropomorphen Wendung letztendlich als Gegensatz zu symbolischem Erfolg auf, beides scheint unvermeidbar aber anknüpfbar und möglicherweise auch verwertbar.

Mit dem Instrument des Kulturwirtschaftsberichts, der 2004 von der „Berliner Initiative für Kreativwirtschaft“ angestoßen ${ }^{5}$ und 2005 in $\mathrm{Zu}$ sammenarbeit mit den damaligen Senatsverwaltungen für Wirtschaft, Arbeit und Frauen sowie für Wissenschaft, Forschung und Kultur umgesetzt wurde, ließ sich nun ein Sektor aus der desolaten Gesamtlage „herausschneiden" - die Kreativwirtschaft. Damit konnten nicht nur AkteurInnen, Netzwerke, vermittelnde Formate (Messen, Großhandel), öffentliche Institutionen und Ereignisse identifiziert werden, die das Feld der nun „offiziellen“ Kreativwirtschaft absteckten. Gleichzeitig war auch ein Instrument geschaffen, mit dem sich fortan eindeutige Zahlen über die Produktivität dieses Sektors liefern lassen. Innerhalb der gesamt gesehen schlechten Wirtschaftslage ließ sich die Dynamik dieses Sektors fortan repräsentieren.

Anstelle des unerklärlichen Widerspruchs tritt nun eine Darstellung, in der die Materialisierung des Scheiterns alter Industrien in Gestalt des industriellen Brachlands zur Voraussetzung für Kulturproduktion und damit für die Kreativwirtschaft wird. Der sich seit den 1990er Jahren zunehmend etablierende Topos des Leerstands/der Brache als Freiraum (Fezer/Wieder 2004: 80), der in der Debatte um die schrumpfenden Städte als Strukturmerkmal aufgegriffen wurde (Oswalt 2004), hat den Ansatzpunkt für eine Semantik geboten, in die ökonomischer Stillstand und eine wachsende Kreativwirtschaft als Kausalzusammenhang eingeschrieben werden konnten: nicht ,aber“, sondern „weil“. Auf diese Weise ist auch „Risiko“ angesprochen, denn diese Rhetorik lagert die Wahr-

5 Der erste Kulturwirtschaftsbericht konnte sich in seinen Wachstumsanalysen auf zwei Berichte zu Wirtschaft und Kultur von 1992 und 2002 beziehen. 
scheinlichkeit des Scheiterns in die alten Industrien aus und bietet mit Verweis auf die bereits erfolgreiche und nun messbare Kulturproduktion einen Ansporn für die (Risiko-)Bereitschaft, sich auf diesen Wirtschaftszweig einzulassen. Dieser Logik folgend und ebenfalls von offizieller Seite unterstützt hat die Dokumentation „Urban pioneers. Stadtentwicklung durch Zwischennutzung“ das dafür notwendige Profil „urbaner Pioniere" skizziert (Overmeyer 2007). Sie ist damit ein weiterer Baustein, der zur individuellen Identifizierung mit diesem Modus der Risikobereitschaft beiträgt und Aufschluss über die veränderte Wahrnehmung und Bewertung risikobehafteter unternehmerischer Kulturproduktion im Stadtraum gibt.

Obwohl die Brachen und Leerräume auch in Berlin knapper werden und die Mieten spürbar steigen, hat sich dieses Bild auch aufgrund der oben beschriebenen Verschiebung der Rhetorik etabliert: Es basiert zum Teil auf noch immer realisierbaren Erfahrungen, zum Teil gehören diese aber schon der Vergangenheit an; es wird als grundlegendes und modellbildendes Paradox in die kulturpolitische Debatte hineingetragen (Göhler 2006: 201f.) oder - von „urbanen Pennern“ über die „,digitale Bohème“ bis zu den „urbanen Pionieren“ (Bunz 2006; Friebe/Lobo 2006; Overmeyer 2007) - in szenewirksame Role-models von Risikobereitschaft übersetzt. Diese Bilder führen ein Eigenleben, sie eilen der Stadt voraus und erzielen entsprechende Effekte (Rehberger 2009). ${ }^{6}$ Mit Blick auf die Attraktivität Berlins für Kreative und deren Risikobereitschaft legen diese Bilder eine hohe Wahrscheinlichkeit, Fuß fassen zu können, nahe und ermutigen zu einer entsprechenden Entscheidung, sich in dem wirtschaftlich sehr schwachen Umfeld niederzulassen.

\section{Ausblick}

Das Sich-Einrichten im Risiko der Kreativwirtschaft, die häufig auch geäußerte Zufriedenheit über den Grad an Selbstverwirklichung im Kontext entgrenzter Arbeit und eines risikoreichen Lebens von Seiten der AkteurInnen - und sei sie ein Schutzmechanismus - müssen empirisch ernst genommen werden. Denn eine ethnografisch-kulturwissenschaftliche Forschungsperspektive hat zum Ziel

6 So verwies jüngst der Vizepräsident der Frankfurter Städelschule auf die vergleichbar niedrigen Lebenshaltungskosten in Berlin, um die Stadt Frankfurt zu mahnen, ,ihre“ Kreativen nicht nur (sehr gut) auszubilden, sondern sie auch durch die Ermöglichung ähnlicher Lebensbedingungen in der Stadt zu halten. 
über die Vor- und Nachteile dieser Beweglichkeit zu informieren. Es bedeutet andererseits, diese Akteurssicht nicht auf Meinungsäußerungen und die Erhebungsmethode nicht auf Interviews zu reduzieren, sondern sich durch Beobachtung und Datenanalyse ein eigenes Bild von den Ursachen und Folgen einschließlich der Langzeitfolgen - flexiblisierter Arbeits- und Lebensweisen zu machen. Nur dann ist es möglich, etwas über Situationsverklärungen und illusionäre Hoffnungen, aber auch über eventuelle unnötige Befürchtungen oder das Übersehen von Flexiblisierungschancen herauszubekommen, was dann zum Eruieren der kulturellen Hintergründe für Fehleinschätzungen oder selektive Wahrnehmung weiterführen kann. (Warneken 2006: 128f.)

Mit dem Forschungsfeld Risiko innerhalb der Kreativwirtschaft ist außerdem eine Beobachtungsperspektive eingezogen, die Aufschluss darüber geben kann, wie Entscheidungen, Unsicherheiten und Wahrscheinlichkeiten individuell in ein alltagstaugliches Verhältnis gebracht werden können. Darüber hinaus eröffnet diese Perspektive aber auch die Möglichkeit, das Abwägen eines individuell passenden Verhältnisses der Risikoelemente nicht als individuell bestimmt zu verstehen, sondern die kollektiven, gesellschaftlichen Anteile darin zu erkennen. In dieser Hinsicht kann ethnografisch-kulturwissenschaftliches Wissen über kulturelle Praktiken im Zusammenhang von Risiko und Risikobereitschaft (alltagstaugliche Verhältnisse zwischen Entscheidung, Unsicherheit und Wahrscheinlichkeit herzustellen) auch für Fragen der „Steuerung“ von Interesse sein. Denn es kann zu einer Stärkung dieser Praktiken auf der Grundlage von geteiltem Wissen beitragen, aus individuellen Lagen, kollektive Interessen werden zu lassen. Während das unternehmerische Selbst nämlich u. a. deshalb alleine dasteht, weil es in einem ständigen Abgrenzungsdruck der „Norm der Individualität“" entsprechen müsse (Bröckling 2007: 68), ließen sich hier Beobachtungen darüber auswerten, wie individuelle Risikobereitschaft in gemeinsame Interessen übersetzt werden kann und daraus Praktiken der Interessensvertretung werden könnten. Dass die bestehenden Instrumente, wie bspw. die Künstlersozialkasse oder gewerkschaftliche Beratungsleistungen für Freiberufliche, zum „Abbau von Lebensrisiken“ (Friebe/Lobo 2006: 285) für einen erweiterten Kreis Betroffener nicht ausreichen werden, liegt nahe; ob das Modell der Kulturgesellschaft realisierbar ist, muss sich noch erweisen, die Aufgaben dafür sind skizziert (Göhler 2006). Szenen, temporäre Kooperationen, Netzwerke sind bis heute nur selten in der Lage auch politisch nachhaltige Effekte für ihre Belange zu erreichen. Eine ethnografisches Wissen einbeziehende „Steuerung“ der Alltagssituation von Kreativen in der Kreativwirtschaft könnte aber in diese Richtung wirken. 


\section{Literatur}

Beck, Ulrich (2007): Weltrisikogesellschaft. Auf der Suche nach der verlorenen Sicherheit, Frankfurt/Main: Suhrkamp.

Berking, Helmuth/Löw, Martina (Hg.) (2008): Die Eigenlogik der Städte. Neue Wege für die Stadtforschung, Frankfurt/Main: Campus Verlag.

Bröckling, Ulrich (2007): Das unternehmerische Selbst. Soziologie einer Subjektivierungsform, Frankfurt/Main: Suhrkamp.

Bunz, Mercedes (2006): „Meine Armut kotzt mich an“, In: zitty 4: S. 17-19.

Deutscher Bundestag (2007): Schlussbericht der Enquête-Kommission „Kultur in Deutschland“, Drucksache 16/7000, 11.12.2007.

Färber, Alexa (2008): „Flourishing Cultural Production in Economic Wasteland: Three Ways of Making Sense of a Cultural Economy in Berlin at the Beginning of the Twenty-first Century“. In: Heßler, Martina/Zimmermann, Clemens (Hg.), Creative Urban Milieus, Frankfurt/Main: Campus Verlag, S. 409-428.

Fesel, Bernd/Söndermann, Michael (2007): Culture and Creative Industries in Germany, Bonn: German Commission for UNESCO.

Fezer, Jesko/Wieder, Axel John (2004): „Raum begrenzter Möglichkeiten - Stadtentwicklung in Berlin nach 1989“. In: Komplex Berlin. Katalog der 3. Berlin Biennale. Köln: Walther König, S. 73-82.

Florida, Richard (2002): The Rise of the Creative Class. And How It's Transforming Work, Leisure, Community and Everyday Life, New York: Basic Books.

Friebe, Holm/Lobo, Sascha (2006): Wir nennen es Arbeit. Die digitale Bohème oder: Intelligentes Leben jenseits der Festanstellung, München: Heyne.

Friedrichs, Jürgen (1998): „Soziale Netzwerke und die Kreativität einer Stadt". In: Albrecht Göschel/Volker Kirchberg (Hg.), Kultur in der Stadt: stadtsoziologische Analysen zur Kultur, Opladen: Leske + Budrich, S. 145-163.

Göhler, Adrienne (2006): Verflüssigungen. Wege und Umwege vom Sozialstaat zur Kulturgesellschaft, Frankfurt/Main: Campus Verlag.

Hess, Sabine/Moser, Johannes (Hg.) (2003): Kultur der Arbeit - Kultur der neuen Ökonomie. Kulturwissenschaftliche Beiträge zu neoliberalen Arbeits- und Lebenswelten, Kuckuck. Notizen zur Alltagskultur, Sonderband 4, Graz: Institut für Volkskunde und Kulturanthropologie.

Heßler, Martina (2007): Die kreative Stadt. Zur Neuerfindung eines Topos, Bielefeld: transcript Verlag. 
Heßler, Martina/Zimmermann, Clemens (Hg.) (2008): Creative Urban Milieus. Historical Perspectives on Culture, Economy, and the City, Frankfurt/Main: Campus Verlag.

IHK Pressemitteilung, Berlin 7.6.2002.

Krätke, Stefan: Medienstadt (2002). Urbane Cluster und globale Zentren der Kulturproduktion, Opladen: Leske + Budrich.

Kulturwirtschaft in Berlin: Entwicklung und Potenziale, Hg. von Senatsverwaltung für Wirtschaft, Arbeit und Frauen in Berlin, Senatsverwaltung für Wissenschaft, Forschung und Kultur. Berlin 2005.

Kokot, Waltraud (Hg.) (2000): Kulturwissenschaftliche Stadtforschung: Eine Bestandsaufnahme, Berlin: Reimer Verlag.

Lange, Bastian: Die Räume der Kreativszenen. Culturepreneurs und ihre Orte in Berlin, Bielefeld: transcript Verlag 2007.

Manske, Alexandra (2008): „Prekarisierung auf hohem Niveau oder: Risikolage Kreativarbeit“. In: Heinricht-Böll-Stiftung (Hg.), Kreativen:Wirkung. Urbane Kultur, Wissensökonomie und Stadtpolitik, Berlin: Heinrich Böll Stiftung: S. 48-52.

Manske, Alexandra (2007): Prekarisierung auf hohem Niveau. Eine Feldstudie über Allein-Unternehmer in der IT-Branche, München: Rainer Hampp Verlag.

Metzler, Birgit (2008): „Creative Milieus: Concepts and Failures“. In: Heßler, Martina/Zimmermann, Clemens (Hg.), Creative Urban Milieus, Frankfurt/Main: Campus Verlag, S. 379-408.

O’Connor, Justin (2005): „Creative Exports: Taking ,Cultural Industries“ to St Petersburg“". In: International Journal of Cultural Policy 11, S. 45-59.

Oswalt, Philipp (2004): Schrumpfende Städte. Band 1: Internationale Untersuchung, Ostfildern-Ruit: Hatje Cantz Verlag.

Overmeyer, Klaus/Berlin Senatsverwaltung für Stadtentwicklung (Hg.) (2007): Urban Pioneers. Stadtentwicklung durch Zwischennutzung, Berlin: Jovis Verlag.

Rehberger, Tobias (2009): „Mein 2009“. In: Journal Frankfurt 1, S. 46.

Schmidt-Lauber, Brigitta (2007): „Feldforschung. Kulturanalyse durch teilnehmende Beobachtung“. In: Göttsch, Silke/Lehmann, Albrecht (Hg.), Methoden der Volkskunde. Positionen, Quellen, Arbeitsweisen der Europäischen Ethnologie, Berlin: Reimer Verlag, S. 219248.

Schmidt-Semisch, Henning (2004): „Risiko“. In: Bröckling, Ulrich/ Krasmann, Susanne/Lemke, Thomas (Hg.), Glossar der Gegenwart, Frankfurt/Main: Suhrkamp, S. 222-227. 
Schwanhäußer, Anja (2008): „Raumästhetik und Raumlabore: Zur kulturellen Einbettung kreativen Schaffens im Neuen Berlin“. In: Zeitschrift für Kulturwissenschaften 1, S. 114-116.

Scott, Allen J. (2000): The Cultural Economy of Cities. Essays on the Geography of Image-Producing Cities, London: Sage.

Seifert, Manfred/Götz, Irene/Huber, Birgit (Hg.) (2007): Flexible Biografien. Horizonte und Brüche im Arbeitsleben der Gegenwart, Frankfurt/Main: Campus Verlag.

Stadt und Kreativität: „Ein Konzept als Perspektive und Effekt. Eine Diskussion zwischen Stadtforschenden“. In: Zeitschrift für Kulturwissenschaften 1 (2008), S. 106-132.

Steets, Silke (2008): „Wir sind die Stadt!“”: Kulturelle Netzwerke und die Konstitution städtischer Räume in Leipzig, Frankfurt/Main: Campus Verlag.

Thibaut, Matthias (2003): „Arm, aber sexy: Wowereit warb in London für Berlin“. In: Der Tagesspiegel 4.12.2003.

Vogt, Sabine (2005): Clubräume - Freiräume, musikalische Lebensentwürfe in den Jugendkulturen Berlins, Kassel: Bärenreiter Verlag.

Waldermann, Anselm (2005): „Unternehmer glauben an die Zukunft“. In: Der Tagesspiegel 24./25.3.2005.

Warneken, Bernd Jürgen (2006): Die Ethnographie popularer Kulturen. Eine Einführung, Wien/Köln/Weimar: Böhlau Verlag. 


\section{Kritik der Kreativindustrien}

\section{SEBASTIAN OLMA}

Der Topos der Kreativindustrien stellt den Versuch dar, ein recht unüberschaubares Gemenge aus emergenten sozial-ökonomischen Strukturen, städteplanerischen Fantasien, Immobilienspekulation, kommunalpolitischen letzten Hoffnungen, akademischem Marketing - um nur einige der wichtigsten Komponenten zu nennen - begrifflich zu fassen. Die folgenden Anmerkungen sind dazu gedacht, ein wenig Ordnung in das Wirrwarr der Argumente zu bringen. Dabei ist es nicht das Ziel, letztgültig zu klären, worum es denn nun wirklich bei dem Phänomen Kreativindustrie geht. Vielmehr soll versucht werden, sich den Creative Industries über eine kritische Bestandsaufnahme der Kritik zu nähern. Dem liegt der Gedanke zugrunde, dass das heterogene Feld vielleicht am effektivsten abzustecken ist, wenn man sich anschaut, was im Zusammenhang mit den Kreativindustrien denn eigentlich im Einzelnen bemängelt wird. Dem sich daraus ergebenen Bild sollen am Ende dieser Ausführungen ein paar Gedanken über Kreativität und Macht im neoliberalen Zeitalter nachgeschickt werden.

\section{Bullshit Britain: Kritik der kreativen Spin Doctors}

Die Geburtsurkunde der Kreativindustrien ist britischer Herkunft und wurde 1998 (mit einem Upgrade in 2001) vom Kultusministerium der Blair-Regierung ausgestellt. Gemeint ist das sogenannte Task Force Mapping Document, welches einen post-industriellen Supersektor, bestehend aus Kunst, Kultur und Softwareindustrie, ins Leben dekretierte (DCMS 1998/2001). Zwar gab es mit der Creative Nation Initiative be- 
reits 1994 einen australischen Vorläufer, aber dieser findet in der Literatur kaum Beachtung. Die zentrale Idee hinter der britischen Initiative war, dass man in der globalisierten Wertschöpfungskette nur dann in der Pool-Position bleiben könne, wenn man sich als Nationalökonomie auf Kreativität und Innovationsfähigkeit spezialisieren würde.

Diese These, entwickelt von Figuren wie dem zu New Labour bekehrten Ex-Marxisten Charles Leadbeater, nahm an, dass sich die etwa von Jean Baudrillard oder Guy Debord vorausgesagte Emanzipation der semiotischen Ebene der Ware (das Bild/Spektakel des Kapitals) in eine aktive Wirtschaftspolitik umsetzen ließe. Peter Mandelson, Blairs ChefSpin-Doctor, erkannte sofort das ideologische Potenzial dieses Ansatzes: Kreativität passte perfekt ins Konzept von New Labour als etwas, das im herkömmlichen Sinne weder Kapital noch Arbeit war, sondern in der Tat einen dritten Weg wies. Und glücklicherweise, so nahm man weiterhin an, hätte Großbritannien wegen der indigenen Kreativität und Innovationskraft seiner Bevölkerung in der Kreativitätsökonomie einen natürlichen Wettbewerbsvorteil. Tony Blair brachte es folgendermaßen auf den Punkt: „We can say with pride that Britain is the ,design workshop of the world"- leading a creative revolution."

Die notwendigen Wirtschaftsdaten, die die These einer postindustriellen Wirtschaftslokomotive zu bestätigen schienen, lieferte das Kultusministerium gleich mit: für 1998 Gewinne in Höhe von $£ 60$ Milliarden/Jahr und für 2000 Schätzungen von $£ 112$ Milliarden/Jahr. Allerdings verdanken sich diese Zahlen vor allem dem Einschluss der Softwareindustrie in die auch ansonsten kreative Buchführung des Ministeriums. Richtig ist, dass cool Britannia Ende der 1990er Jahre das eine oder andere vorzuweisen hatte: Soho hatte gerade der Madison Avenue den Schneid beim Werbegeschäft abgelaufen, die Young British Artists waren kommerziell bereits ziemlich in Fahrt, und es gab ein paar erfolgreiche Popbands. Allerdings, so die einheimischen Kritiker des creativity hype, wurden hier zu keinem Zeitpunkt Größenordnungen erreicht, die annehmen lassen durften, die Kreativindustrie könne die desolate britische Wirtschaft aus ihrer Misere führen. ${ }^{1}$

Hier haben wir ein wichtiges Problem der Kreativindustrie, auf das vor allem Wirtschaftsexperten immer wieder hinweisen: die fehlende ökonomische Grundlage. Wie beispielsweise die Chefökonomen der Tageszeitung The Guardian, Larry Elliot and Dan Atkinson, betonen, gibt

1 In der Tat muss festgestellt werden, dass die unlängst zusammengebrochenen Säulen der Wirtschaft Großbritanniens - nämlich der Finanzdistrikt der City of London und der Immobilienmarkt - sehr wenig mit Kreativität im hier diskutierten Sinn zu tun haben. 
es bis heute in der Tat keinen nennenswerten Kreativitätseffekt in der britischen Wirtschaft (Elliot/Atkinson 2007). Natürlich, so wird argumentiert, gibt es urbane Gebiete, in denen sich kulturell-künstlerisches Unternehmertum konzentriert - East London und Glasgow wären hier als prominente Beispiele zu nennen. Der Versuch, dies anderswo zu wiederholen schlägt jedoch regelmäßig fehl. So verweist der zum Sprachrohr der ökonomisch informierten Linken gewordene Kritiker James Heartfield auf die kurze Halbwertzeit unzähliger art centres und ähnlicher New Labour Fehlinvestitionen zur Anregung des kreativen Sektors und Regeneration postindustriell verwüsteter Städte (Heartfield 2005). ${ }^{2}$

Was die Kritik am fehlenden ökonomischen Fundament betrifft, so ist diese formal zutreffend: Blair \& Co hatten sich ihren neuen Supersektor wirklich kreativ herbeigerechnet. Problematisch ist jedoch die ethische Position, auf deren Grundlage diese Kritik vorgetragen wird: Der sich ausbreitende postindustrielle Kapitalismus sei dekadent und deshalb nicht lebensfähig. So witzeln Elliot und Atkinson beispielsweise über die Ikonen der Kreativindustrie als ,men and women who think that making up jingles and slogans to flog Pot Noodles and similar products is a serious job“ (2007: 73).

Dies ist unterhaltsames Gegengift für Kreativrhetorikgenervte und mag vielleicht sogar eine gute Charakterisierung der englischen Situation sein. Der von der zitierten Kritikversion vorgetragene Ausweg, nämlich die Rückkehr zu einem stärker auf manufacturing beruhende System, ist allerdings im britischen Kontext eher unwahrscheinlich. Zwar gibt es inzwischen einen im Schatten der Finanzkrise ausgearbeiteten Report des Wirtschaftsministeriums, der die ,neuen Herausforderungen und Chancen“ dieses Sektors betont (BERR 2008). Allerdings finden auch die Autoren des Papiers keine schlüssige Antwort darauf, wie dies denn im heutigen England erreicht werden soll.

Außerdem verliert diese Art von Kritik vor lauter Zynismus die Bedeutung des Kritisierten aus den Augen. Hier sind die vom Australischen Medienwissenschaftler Ned Rossiter formulierten Anmerkungen zum Thema etwas ergiebiger. Nach seinem Dafürhalten muss das britische Task Force Mapping Document mit der WTO-Vereinbarung über Trade-Related Aspects of Intellectual Property Rights (TRIPS) in Zu-

2 Im Sommer 2008 sorgte ein Report des renommierten konservativen Think Tanks Policy Exchange im Vereinigten Königreich für Wirbel, weil er ernsthaft vorschlug, die Regenerationsprogramme für Städte wie Liverpool, Bradford, Hull oder Sunderland endlich für gescheitert zu erklären und die Städte aufzugeben (vgl. Leunig/Swaffield 2008). 
sammenhang gebracht werden. Letztere führte ein Kontrollregime ein, mit dem die verstärkte Informatisierung des sozialen Lebens rechtlich in Schach gehalten werden soll. Die These vom Doppelfundament der Kreativen Industrie ermöglicht es, das Phänomen in den weiteren Kontext sozial-ökonomischer Evolution zu stellen. In der Tat ging es auch in der britischen Initiative um die Bildung und Nutzung intellektuellen Eigentums (DCMS 1998/2001), so dass die Annahme angebracht scheint, dass die Kreativindustrie als politisches Programm faktisch auf eine Transformation der Produktionsstrukturen abzielt, und zwar derart, dass Kreativität (die Referenz ist hier auf ,individual creativity, skill and talent") in entsprechenden Eigentumsverhältnisse überführt werden kann. Noch wichtiger an Rossiters Intervention ist jedoch der Hinweis, dass die strukturelle Determination, die derartige Initiativen herstellen, mit den Verhältnissen und Erfahrungen der kreativen ArbeiterInnen abgeglichen werden muss. Nur so ist es möglich, zu einer realistischen Einschätzung des Phänomens der Kreativindustrien zu kommen. In der öffentlich-politischen Debatte spielen derartig radikale Positionen zwar derzeitig noch keine bedeutende Rolle. Trotzdem möchte ich am Ende dieses Texts noch einmal kurz darauf zurückkommen.

Mit Blick auf die Kunstschaffenden im Vereinigten Königreich müssen weitere unmittelbare Folgen der mit den Creative Industries verbundenen Förderpolitik angesprochen werden - auch weil sie mittlerweile ein Problem für Künstler weltweit geworden sind. Gemeint ist die Tatsache, dass vom Kultusministerium unterstützte Kunst positive soziale Relevanz unter Beweis stellen oder kommerziell verwertbar sein musste. Damit wurde Kunst zum Instrument von Sozialpolitik degradiert. Davon profitierte zwar die so genannte community arts-Bewegung, aber einer in ihrem Selbstverständnis autonomen oder gar sozialkritischen Ästhetik war eine derartige Funktionalisierung nicht unbedingt zuträglich. Wie die Soziologen Hesmondhalgh und Pratt betonen, werden Künstler so gezwungen, sich explizit als Unternehmer zu begreifen. Letzteres muss man nicht unbedingt problematisch finden, man muss sich nur darüber im Klaren sein, dass der Künstler, der bewusst Geschäftsmann ist, einer radikal anderen Logik folgt als derjenige, der sich autonom wähnt (Hesmondhalgh/Pratt 2005: 8f.). 


\section{Florida angezählt: Kritik der „kreativen Klasse“}

Ein weiterer wichtiger Moment in der Welt der Kreativindustrie war die Publikation von The Rise of the Creative Class im Jahre 2002. Sein Autor, der US-Amerikanische Stadtsoziologe Florida, wurde beinahe über Nacht zum Superstar und Coolness-Guru im bis dato wenig schillernden Feld urbaner Theorie und Städteplanung. Floridas zentrale These bestand in der Behauptung, eine neue, sich im Aufstieg befindliche Klasse hochausgebildeter Kreativlinge entdeckt zu haben, um die sich im postindustriellen Zeitalter bereits alles dreht und zukünftig weiterhin drehen wird. Obwohl kreative Menschen schon immer eine wichtige Rolle für die kapitalistische Entwicklung gespielt hätten, wäre deren Zahl und Einfluss in den letzten Jahrzehnten derartig gestiegen, dass Sie heute ungefähr $30 \%$ der arbeitenden Bevölkerung ausmachten (Anwälte und Bankiers gehören ebenso zu Floridas Kategorie wie Künstler, Entertainer und Architekten). Tendenz steigend. Damit, so Florida, ,ist die kreative Klasse zur herrschenden Klasse der Gesellschaft geworden“ (Florida 2002: ix).

Damit verschärfte Florida die britische Debatte, indem er die dort vorgebrachten Argumente in eine pseudoradikale Klassenkampfrhetorik verpackte. Nimmt man seine Einlassungen ernst, so hat dies einschneidende gesellschaftspolitische Folgen: Als entscheidender Faktor zukünftigen wirtschaftlichen Wohlergehens muss die kreative Klasse erst angesiedelt und dann gehegt und gepflegt werden. Kommunalpolitiker und Firmenleitungen müssen aktiv für das richtige people climate sorgen, sonst bleibt die kreative Klasse weg und damit der wirtschaftliche Erfolg aus. Vor allem bedeutet dies, dass herkömmliche Kommunalpolitik über den Haufen geworfen werden muss, oder zumindest verschoben, und zwar auf das neue interkommunale Wettbewerbsterrain der drei T's: Talent, Technologie und Toleranz. Laut Florida sind das nämlich die Parameter, die das Feld abstecken, auf dem Städte und Regionen um eine erfolgreiche wirtschaftliche Zukunft wetteifern. Das wichtigste der T-Triade ist zweifellos Talent. Glaubt man Florida, so werden urbane Erfolgsgeschichten in Zukunft nur von denjenigen geschrieben, die die neue humane Ressource der talentiert-kreativen Klasse an sich binden können.

Floridas Thesen stießen auf offene kommunalpolitische Ohren überall in den Vereinigten Staaten. Dies hatte vor allem zwei Gründe. Der erste ist praktischer Natur: Wie auch in Großbritannien, gab Floridas Entwicklungsmodell postindustriell desillusionierten Kommunalpolitikern neue Hoffnung für ihre wirtschaftlich heruntergekommenen Städte. 
Darüber hinaus sind Investitionen in Kunst und Kultur (zur Herstellung des kreativen Ökosystems) preiswerter zu haben und medienwirksamer $\mathrm{zu}$ inszenieren als das Klein-Klein nachhaltiger Wirtschaftspolitik (Peck 2005). Der zweite Grund für Floridas Erfolg ist eher epistemologischer Natur: so bediente der Soziologieprofessor das gesamte Arsenal der Management-Binsenweisheiten, die im Laufe der 1990er Jahre ihren Weg in den offiziellen politischen Diskurs gefunden hatten. Peter Druckers Erkenntnis (die eigentlich aus den 1960er Jahren stammte), dass in der Wissensgesellschaft eben vor allem Wissen als ökonomischer Faktor relevant war, polte Florida einfach auf Kreativität um, kombinierte sie mit der Wettbewerbsgeographie von Rosabeth Moss Kanter und Kenichi Ohmae, und verpackte das Ganze in New-Economy-Rhetorik à la Fast Company. Das so geschnürte Paket, versehen mit einer cleveren Marketingstrategie, machte Florida zum Guru und seine kurzerhand gegründete Beraterfirma Catalytics zu einem äußerst rentablen Unternehmen.

Ein oft zitiertes Kuriosum aus Floridas Feder war der so genannte gay-index, demzufolge es eine Korrelation zwischen der Größe der Homosexuellenpopulation und technologieintensivem Wachstum gäbe (Schwule als Kreativitätsbarometer). Die rechte Kritik war außer sich, selbst seriösere Kommentatoren, wie Steven Malanga vom konservativen Manhattan Institute, witterten eine linke Verschwörung, da hier unter dem Deckmantel von Toleranz traditionelle Werte (family values usw.) entsorgt werden würden und außerdem noch ein aktives Eingreifen der Politik in den Markt zur Schaffung des richtigen people climate propagiert wurde. Ernstzunehmen ist Malanga's Kritik allerdings dort, wo sie darauf verweist, wie störrisch die US-Amerikanische Empirie im Angesicht der Kreativen Klasse bleibt: Die wirtschaftlich erfolgreichsten Städte (gemessen an Indikatoren wie Beschäftigung, Bevölkerungswachstum, Bildung erfolgreicher Unternehmen) bleiben eben doch eher konservative Zentren wie Las Vegas oder Memphis, also Städte, die in Floridas Kreativitätsliste ganz unten stehen (Malanga 2004).

Für die akademisch fundierte US-Kritik verdeutlichte der gay-index die Tatsache, dass Floridas Argumentation eher auf schrägen Korrelationen als auf einer handfesten Kausalargumentation beruht (Marcuse 2003; Sawicki 2003). Floridas schärfster Kritiker in dieser Hinsicht ist zweifellos sein Landsmann und Fachkollege Jamie Peck, der eine detaillierte Punkt-für-Punkt Kritik von The Rise of the Creative Class abgeliefert hat. Wie er überzeugend darlegt, ließe sich der von Florida behauptete Mechanismus, dass nämlich eine lebendige Kunst- und Kulturszene wirtschaftliches Wohlergehen schafft, genauso gut und vielleicht sogar überzeugender, umdrehen (Peck 2005: 755). 
So brillant und fundiert solche Kritik an Floridas Thesen auch sein mag, sie bleibt in der Praxis ohne Wirkung. Die oben genannten Erfolgsfaktoren haben Florida und seine Idee der kreativen Klasse zu so etwas wie den Joker urbaner Reformer gemacht. Bleibt abzuwarten, ob es im Zuge der Finanzkrise hier eine Änderung der Spielregeln geben wird.

\section{Kreativität auf Welttournee: Kritik der Global Creative Industries}

Kombiniert mit Floridas sonnigen Thesen ist Blairs policy initiative schnell zum Exportschlager geworden - ein Erfolg mit dem selbst die Initiatoren wohl so nicht gerechnet hatten. Seit Beginn des neuen Millenniums verschrieben sich immer mehr Regierungen der kreativen Industriepolitik in dem Glauben, hier einen veritablen Weg in die postindustrielle Zukunft gefunden zu haben. Kanada, Neuseeland, Ostasien, Brasilien und Westeuropa sind hier zu nennen.

In der letzten Zeit ist vor allem auch China auf dem Radar der internationalen Kreativitätsexponenten aufgetaucht. Dies hat natürlich einiges mit dem allgemeinen China-Hype zu tun, aber auch mit der Tatsache, dass die Kreativindustrien seit dem 11. Fünfjahresplan offizielle Politik der Volksrepublik geworden sind. Wie der australische Soziologe und China-Experte Michael Keane (2007) zeigt, hat die chinesische Parteiführung nach sehr kurzer Bedenkzeit das Konzept der Kreativindustrien angenommen und entsprechende Politikinitiativen gestartet. Seitdem wird der Kreativitätssektor vor allem in Chinas Metropolen wie Beijing, Shanghai, Chongqing, Guangzhou, Nanjing, Chengdu, und Tianjin entwickelt (Tong 2006; Qidi 2006).

Oft wird diese Entwicklung mit der Hoffnung nach politischer, sozialer und kultureller Liberalisierung verbunden (z. B. Xiaoming 2006; Keane 2007.) Allerdings ist am Beispiel Shanghai recht konkret gezeigt worden, dass Kreativindustrie und drakonische Ordnungspolitik einander keineswegs ausschließen müssen. Offensichtlich lassen sich bohemian zones auch derart kontrolliert einrichten, dass dadurch die allgemeine moralische Ordnung nicht aus den Fugen gehoben wird (Lee 2004). Den Offiziellen scheint die Vision von kreativen Jungunternehmern, die sich kommerziell selbstverwirklichen, jedenfalls keinen großen Schrecken einzujagen. Aber auch wirtschaftlich ist die kreative Wende opportun für die chinesische Führung.

Wie der amerikanische Kulturwissenschaftler Andrew Ross (2007: 33) kommentiert, ist Chinas weiterer Aufstieg auch davon abhängig, inwieweit es beim Schaffen geistigen Eigentums von der Imitation auslän- 
discher Innovation auf hauseigene Neuerung umstellen kann. Dementsprechend hat Premier Wen Jiabao 2006 die „unabhängige Innovation“ (zizhu chuangxin) zur ökonomischen Entwicklungsstrategie der nächsten fünfzehn Jahre erklärt. Natürlich geht es auch hier darum, an die Spitze der globalen value chain zu gelangen. Welchen Beitrag die Kreative Industriepolitik dabei leisten kann, bleibt abzuwarten. Gleiches gilt für die sich noch im Formierungsprozess befindende Kritik. ${ }^{3}$

Zurück in Europa muss konstatiert werden, dass die interessanteren Kommentare der Kritikergemeinde solche sind, die sich an konkreten regionalen Fallstudien abarbeiten. Der italienische Digitalaktivist und -kritiker Matteo Pasquinelli nimmt sich beispielsweise des Regenerationsprozesses Barcelonas an. Zu Hilfe kommt ihm dabei der britische Geograph David Harvey (2001), der in Barcelonas Aufstieg in die Prominentenliga europäischer Städte das Ergebnis eine konzertierte Aktion sieht, bei der sich Stadtregierung, zivilgesellschaftliche Organisationen und Privatinteressen neoliberal vereinten, um aktiv und massenhaft symbolisches Distinktionskapital anzuhäufen.

Dagegen ist per se selbstverständlich nichts einzuwenden. Allerdings ist Harvey zuzustimmen, wenn er kritisiert, dass der später daraus gezogene Gewinn nur an einige wenige Immobilienspekulanten geht. Und man muss ihm sogar Recht geben, wenn er dafür das etwas verstaubte Konzept des rent seeking aus dem Archiv holt. Gemeint ist damit die Tatsache, dass das gemeinschaftlich aufgebaute symbolische Kapital die Immobiliengemeinde Barcelonas in die Lage versetzt, eine Art Monopolzuschlag auf Verkauf oder Vermietung Ihres Besitzes einzufordern, denn das hippe Barcelona gibt es schließlich nur einmal.

Der Gedanke, den Pasquinelli den Betrachtungen Harveys beifügt, dreht sich um die Frage, inwieweit man sich vor Ort solchen Vereinnahmungen entziehen kann. Denn ist es nicht so, fragt der Autor, dass an der „kreativen Halluzination“ Barcelonas nicht nur die sich beispielsweise im Umfeld der MACBA angesiedelten Kreativindustrien mitarbeiten, sondern auch die Hausbesetzer, Anarchisten und sonstige Aktivisten, die versuchen, sich gegen ihre Vertreibung im Zuge urbaner Regeneration zur Wehr zu setzen, ihren kulturellen Beitrag zur neoliberalen Rentenökonomie leisten? Das hier in verschärfter Form aufgeworfene Problem unfreiwilliger Komplizenschaft übersetzt ein zentrales Anliegen der italienischen Autonomiebewegung in die Situation des (mehr oder weniger) kreativen Prekariats: Ist ein „Exodus“ aus dem derzeitigen Verwer-

3 Die Ergebnisse eines internationalen Counter-Mapping Creative Industries Projekts waren zu Redaktionsschluss noch nicht veröffentlicht. 
tungssystem möglich? Die Frage ist von großer Wichtigkeit, auch wenn sie bis auf weiteres unbeantwortet bleibt.

Auch das kleine Amsterdam ist seit einigen Jahren ein big player im Zirkus der Creative Industries. Hier gibt es eine Allianz aus Politik, Wirtschaft und künstlerisch-kulturellen Öffentlichkeitsinstitutionen, die auch und vor allem durch die Einrichtung so genannter cultural clusters die Ausbildung der Kreativindustrien betreiben. Der niederländische Sozialgeograph Hans Mommaas (2004) hat einige dieser Clusters studiert und entdeckt darin die nächste Stufe der fortschreitenden Instrumentalisierung von Kunst und Kultur als Stadtentwicklungsressourcen. Allerdings, so urteilt der Professor, ist es alles in allem ein Schritt in Richtung einer offeneren, inklusiven kommunalen Planungspolitik.

Aktivist und Herausgeber der Zeitschrift Flexmens Merijn Oudenampsen hat eine Untersuchung zum konkreten Fall Amsterdam vorgelegt, die nicht nur dieser Einschätzung widerspricht, sondern auch Floridas These vom Zusammenhang von kreativer Klasse und dem big T von Toleranz widerlegt. Kreativindustrie funktioniert in Amsterdam im Kontext einer gründlichen Florida-inspirierten Metamorphose unter dem Motto „I Amsterdam“. Laut Oudenampsen ist „I Amsterdam“ allerdings eher der Schlachtruf einer neoliberalen Abrechnung mit dem sozialprogressiven planerischen Vermächtnis der Stadt. War Stadterneuerung in Amsterdams jüngerer Geschichte eng verbunden mit reformerischen Ideen, wie Aufwärtsmobilität, Emanzipation und, in der Tat, Integration, so scheint die derzeitige Politik der kreativen Stadt ein willkommener Anlass, um all dies endlich über Bord zu werfen.

Richtete sich städtische Planungspolitik in der Vergangenheit vor allem auf die unteren sozialen Schichten, so steht heute die (kreative) Mittelklasse im Visier der Entwickler. Kreativer face lift bedeutet hier in der Regel Abriss und Privatisierung sozialer Wohnungsbausubstanz. Damit verbunden ist das neue Prinzip geographischer Umverteilung von Armut, das einhergeht mit der offen rassistischen Vertreibung von nichtweißen Migranten aus als potentiell kreativ demarkiertem Urbanterritorium. Beim kreativen Upgrade des Viertels denken Stadtteilverwaltungen verstärkt an das Entfernen türkischer/marokkanischer Geschäfte (sowie an die Liquidierung von Coffeeshops). Natürlich werden hier Alternativen angeboten, irgendwo in der Peripherie, wo man keiner planerischen Kreativitätsphantasie im Wege steht. Man muss sicherlich nicht betonen, dass diese Art der Politik nicht viel mit Toleranz zu tun hat. Bleibt abzuwarten, ob diese Politikpraxis dem ostentativ liberalen Amsterdam nicht über kurz oder lang das teuer produzierte Image verderben wird. 
Eine durchaus globale Perspektive bietet der US-amerikanische Kulturkritiker Brian Holmes. Aufhänger seiner Kritik ist ein Streifzug durch North-Carolinas Research Triangle Park, der in den 1950er Jahren als Refugium für Forschungslaboratorien großer US-Konzerne angelegt wurde. Ziel der Politik war regionale Wiederbelebung, während die sich ansiedelnden Unternehmen auf hochfrequente Patentproduktion aus waren. Laut Holmes gab der Research Triangle Park nicht nur der umliegenden Region (das Research Triangle um die Universitätszentren Chapel Hill, Durham und Raleigh) seinen Namen, sondern ist auch eine Art Strukturmodel der Kreativindustrien. Bei allen oberflächlichen Unterschieden ging es damals wie heute um staatlich geförderte Schaffung urbaner Räume, in denen gesellschaftliche Kreativität effektiv industriell abgeschöpft werden kann. Nur geht dies eben heute wesentlich effizienter und umfassender vor sich.

Als das zentrale Relais für diese Tendenz in den USA, aber auch weltweit, kennzeichnet Holmes die zunehmende Privatisierung universitärer Wissensproduktion. Von entscheidender Bedeutung dafür ist die Praxis der Patentierung von mit öffentlichen Mitteln gewonnnen Forschungsergebnissen, die seit dem Bath-Doyle Act von 1980 gesetzlich kodifiziert ist. Die WTO sorgt dafür, dass das so erschlossene geistige Eigentum auch globalisiert und die entsprechenden Profite vervielfacht werden können. Das Research Triangle dient Holmes als dreifaltige Metapher dieses Prozesses: UNC Chapel Hill als Zuhälterin der Industrie, die ihre Studenten als willige Forschungshuren anpreist, Duke als Exporteur der neoliberalen Universitätspraxis in Richtung Singapur und Asien, und schließlich NCSU Raleigh, die ihre Seele an das Pentagon verkauft: hier gewinnt ein militärisch-akademisch-industrieller Komplex an Gestalt, der die Kreativität aller Beteiligten kidnappt - im besten Fall zur Profitmaximierung und im schlechtesten für den nächsten Eroberungskrieg.

Natürlich ist Holmes' Argument sehr auf die USA gerichtet und leicht dramatisiert. Aber eben nur leicht. Auch in Europa ist es nicht mehr besonders weit her mit der wissenschaftlichen Unabhängigkeit der Universitäten. Verschulung, Drittmittelzwang, Managerialisierung, Abbau irrelevanter Geistes- und Sozialwissenschaften: Die neoliberale Reise hat auch hier längst begonnen. Die Frage ist allerdings, was der Niedergang der Universität mit den Kreativindustrien zu tun hat? Dazu ist einerseits zu sagen, dass die Art und Weise, in der „Industrie kreativ gemacht wird“ an dieser Stelle sicherlich relevant ist. Andererseits ist Holmes der Überzeugung, dass die Relevanz der Kreativindustrien per se rein ideologischer Natur ist. Sie sind die ,touchy-feely side of technology“ (Holmes 2007: 184) und dienen lediglich der gesellschaftlichen 
Legitimation der beschriebenen Praxis. Die Kreativindustrien besorgen demnach die Gleichsetzung von Kreativität und Unternehmertum gewissermaßen auf Volksniveau. Die Zivilgesellschaft kann in den staatlichprivaten Dauerarrest des Neoliberalismus gelockt werden, weil die Kreativität als unternehmerische Tätigkeit individuell normalisiert ist.

So wichtig es ist, auf die industrielle Vereinnahmung zivilgesellschaftlicher Kreativität hinzuweisen, so wenig überzeugt die Reduktion der Kreativindustrien auf ein rein ideologisches Phänomen. Mag sein, dass die Verbreitung kultureller Klein(st)unternehmer bei der Neoliberalisierung der Köpfe hilft, aber doch bitte etwas weniger glatt und funktionalistisch, als von Holmes dargestellt. Nicht zuletzt die in diesen Notizen besprochene Kritik an den Kreativindustrien macht deutlich, dass sich das Phänomen nicht in einer Feigenblattfunktion für großindustrielle Raubzüge erschöpft.

\section{Alles Lüge! Kritik des „kreativen Imperativs“}

Seit einiger Zeit wird die deutsche Diskussion über die Kreativwirtschaft unter anderem geprägt von Holm Friebes und Sascha Lobos Wir nennen es Arbeit (Friebe/Lobo 2006), das den Begriff der „digitalen Bohème“ im Feuilleton und am Stammtisch etablierte. Was den relativ jungen Autoren in vorzüglicher Manier gelang, war Floridas creative-class-These auf Berlin zu münzen und mit dem an eine ganze Elterngeneration gerichteten Erklärungsversuch, warum das mit der Banklehre doch nicht geklappt hat, zu verbinden. Der entscheidende Vorteil des Werks gegenüber Floridas professoralen Einlassungen ist Authentizität: hier sind zwei, die nicht nur über digitale Bohème schreiben, sondern selber dazugehören.

Da die hauptberuflichen Bohemiens ziemlich unverblümt ihre freelance-Unterbeschäftigung zelebrieren, scheinen sie genau zu bestätigen, was die diskursanalytisch trainierte akademische Kritik im deutschsprachigen Raum schon lange wusste: wir befinden uns unter der Fuchtel eines „kreativer Imperativs“ (Osten 2003; Bröckling 2003), mithin einem hegemonialen Kreativitätsdiskurs, der individuelle Unternehmerschaft (Stichwort: Ich-AG) als sexy darstellt und - semantisch vermittelt - auch erfahrbar macht. Selbst wenn man wollte (und wer wollte schon nicht sexy und kreativ sein), kann man sich dem kreativen Denk- und Handelszwang nicht entziehen. Junge Unternehmer und solche, die sich dafür halten, werden so zu Diskursopfern, die Ihre Haut preiswert, un- 
organisiert, dafür aber gutgelaunt (zumindest für eine gewisse Zeit) zu Markte tragen.

Diese Argumentation ist insofern adäquat, als sie die Prekarität der neoliberal freigesetzten Subjekte thematisiert. Man sollte sich jedoch davor hüten, hier die Repräsentanten einer Generation von Opfern eines „massenhaften Selbstbetrugs“ (Raunig 2007) zu entdecken: Das Arbeit $=$ Party Pamphlet von Friebe und Lobo lässt sich nicht auf eine globale Kreativarbeitergeneration hochrechnen. Urbane Penner und digitale Bohemiens sind Produkte der Anomalie Berlin. Sicherlich sitzen auch in London und New York Leute mit Laptops im Café. Im Unterschied zu ihren Berliner Kollegen müssen diese allerdings irgendwann zur Arbeit, weil sie sonst ihre Miete nicht bezahlen können. Friebe und Lobo konnten sitzen bleiben und haben sich in der Tat clever selbst zur Brand geschrieben. Vielleicht ist es dann angebracht, über die so zu Ruhm Gelangten als „kreative Superstars“ (Manske 2007) zu lästern; sie zum weberschen Idealtypus einer neuen Ära zu erklären, wäre Nonsens. Denn für die außerhalb der Berliner Stadtgrenzen liegende Welt der real existierenden Kreativindustrie gilt, dass es sich niemand leisten kann, seine eigene Prekarität zu Gunsten irgendwelcher Kreativitätsfloskeln zu ignorieren.

\section{5. Über Kreativität und Macht im neoliberalen Zeitalter}

Im Übrigen ist es nicht nur ziemlich überheblich, Kreativunternehmern und -freiberuflern vorzuwerfen, sie wären einem epistemologischen Schwindel auf dem Leim gegangen, sondern trägt dies auch nichts zur Entschlüsselung des Problems bei. Die Form der Argumentation ist schlichtweg obsolet: Herrschende Diskurse, denen man mit dekonstruktivistischen Textanalysen (die Welt als Text!) effektiv zu Leibe rücken kann, setzen ein relativ stabil strukturiertes Sozialgefüge voraus.

Dass sich dies seit der ,linguistischen Wende“ der 1970er Jahre dramatisch geändert hat, dafür ist nicht zuletzt das Phänomen der Kreativindustrien ein Beleg. In Netzwerkgesellschaften funktionieren Machtprozesse schlichtweg nicht mehr primär diskursiv oder epistemologisch. Neoliberale Steuerungs- und Machtprozesse - von Foucault bereits in den 1970er Jahren antizipiert (Foucault 2004) - sind darauf ausgerichtet, materiale Bedingungen zu schaffen, in denen Produktions- und Kreativitätsprozesse autonom ablaufen können.

Die Kreativindustrien sind gerade deshalb ein so treffendes Beispiel für neoliberale Politik im Sinne Foucaults, weil eben kein „kreativer Im- 
perativ“ existiert. Es gibt kein Programm, keinen Hegemonialdiskurs etwa im Sinne einer kreativen Disziplinarität. Subjekte werden nicht mehr epistemologisch konstituiert: Subjektivierung als wissensgeleitete „Formgebung“ ist nicht das Geschäft des Neoliberalismus. ${ }^{4}$ Vielmehr werden Strukturen geschaffen - beispielsweise die kreative Stadt - in denen sich die Subjekte völlig unterschiedlich (in Differenz) selbstkonstituieren können. Die simultane Erfahrung von unternehmerischer Unabhängigkeit und Prekarität verweist auf eine nichtdiskursive, ja ontologische Neuformation von Machtprozessen: das Vermögen, selbstständig kreativ oder produktiv zu sein, verschränkt sich mit dem Zwang zur Entwicklung des Individualkapitals. Das Verschmelzen dieser zwei essentiellen Machtpole, die Spinoza bereits im 17. Jahrhundert als potentia und potestas markierte und die ihren Weg in die zeitgenössische Kulturkritik über die Arbeiten des italienischen Philosophen Antonio Negri (1990) gefunden haben, verursacht einen Großteil der Probleme beim Begreifen der sozialen Prozesse der Gegenwart. Die Selbstkonstitution des heutigen Subjekts bedeutet, dass der Zwang von potestas sich bereits im kreativen Vermögen von potentia befindet. Damit wird Machtanalyse äußerst schwierig und Demaskierung von Diskursen überflüssig und nutzlos.

Deshalb: Anstatt den Leuten ein falsches Klassenbewusstsein (NeoFoucauldianisch verpackt) vorzuwerfen, wäre es sinnvoller, etwas in die Debatte zu werfen, was den Prekärgewordenen Orientierung bieten kann. Eine „Gebrauchsanweisung für die digitale Bohème“ - vom Penner für Penner - so ein Buch wäre nötig gewesen. Stattdessen gibt es eine Phantomdiskussion zwischen selbsternannten Kreativitätsabgeordneten und einer akademischen Diskursopposition.

Und wenn man den Begriff des kreativen Imperativs mal inhaltlich unter die Lupe nimmt, dann bleibt davon auch nicht viel übrig. Da sich die Exponenten der deutschen Debatte so sehr auf Diskurs und Managementrhetorik einschießen, dürfte ihnen nicht entgangen sein, dass nach der Reengineering- und Outsourcingwelle vor allem der 1990er Jahre heute wahrlich nicht von einem vorherrschenden Kreatitivitätscodex in der Arbeitswelt die Rede sein kann. Will heißen, dass im Bereich der so genannten white-collar work, um die es hier ja wohl geht, in den letzten zwei Jahrzehnten eine systematische Dequalifizierung der Arbeit stattgefunden hat, von der die Population der zahlreichen Call-, Service-, und ähnlichen Centern ein Lied singen kann. Zur Veranschaulichung des

4 Dies falsch verstanden zu haben, ist übrigens auch Brian Holmes' Problem in seiner ansonsten brillanten Studie. 
Sachverhalts kann hier die Arbeit des Forschungskollektivs Kolinko zu Rate gezogen werden, die den Arbeitsalltag in den neuen Arbeitswelten ethnologisch erforscht und dokumentiert hat (Kolinko 2002). Dass die Studie etwas klassenkämpferisch daherkommt, sollte nicht von der Tatsache ablenken, dass hier ein wertvoller Einblick in einen zentralen Sektor des neoliberalen Wirtschaftsgefüges gewährt wird. In den hier untersuchten hyperfordistischen Neofabriken muss man wohl eine Art Antithese zur arbeitsweltlichen Kreativität sehen, zumindest insofern, als hier eine massive Vernichtung von schöpferischer Fähigkeit und Fertigkeit betrieben wird. Die Arbeit des ehemaligen Financial Times Korrespondenten Simon Head (2005) ist hier ebenfalls äußerst aufschlussreich und zwar vor allem deshalb, weil sie nicht Managementrhetorik analysiert, sondern sich anschaut, welche Strukturen durch Reengineering und Outsourcing entstehen. Sein Fazit: die massive Mechanisierung der Arbeitsprozesse, die übrigens nicht nur die Rekruten von Call-Centern in vorformatierte Abläufe presst, sondern die auch hochqualifizierte Fachkräfte bis hin zu Ärzten die Entscheidungen vorschreibt, hat eine Maschine kreiert, die systematisch professionelle Kreativität vernichtet. Was laut Head auf der Strecke bleibt, sind „die erlernten Fertigkeiten, das angesammelte Wissen und die Erfahrung, die Arbeiter und Angestellte in die Lage versetzen, gute Arbeit zu leisten (2005: 169).

Das klingt nicht gerade nach kreativem Imperativ. Hier scheitern die Diskurswächter an ihrem eigenen Programm, da sie der neoliberalen Ideologie genauso auf den Leim gehen, wie sie dies den digitalen Bohemiens unterstellen: Fokussiert auf ein Fragment metropolitaner Jungunternehmer verliert man das Gesamtgefüge aus den Augen, in das sich das Phänomen der Kreativindustrie einordnet.

Der britische Sozialgeograph Nigel Thrift beschreibt das Gefüge des neoliberalen Kapitalismus im Sinne einer Netzwerkökologie in der „Unternehmen zunehmend zu Projektkoordinatoren werden, die ihren normalen Betrieb auslagern, um sich auf die Entwicklung und Implementierung neuer Ideen konzentrieren zu können (Thrift 2006: 287). Der Aufstieg der Kreativindustrien ist wohl ein Zeichen dafür, dass eben auch das Ideenbusiness outgesourced wird, und zwar in den urbanen Raum der Kreativindustrien.

Natürlich ist die derartig funktionalisierte Stadt auch ein Laboratorium, in dem die Qualität und Effektivität frei schwebender „kreativer Arbeit" (Rossiter 2006) getestet werden kann. Allerdings ist Rossiters Begriff auch gewählt, um das politische Potenzial der Netzwerkarbeiter vor allem hinsichtlich ihrer Fähigkeit zur Selbstorganisation zu betonen. Das heißt, für Rossiter zeigt sich in den Kreativindustrien auch die Chance einer Öffnung hin zu nichtrepräsentativen Organisations- und 
Politikformen. Das Standardbeispiel an dieser Stelle ist das 2004 gegründete Netzwerk Coordination des Intermittents et Précaires in dem französische Medienarbeiter für die Verbesserung ihrer Existenzbedingungen kämpfen. Obwohl es sich dabei in der Tat nicht um eine klassische Gewerkschaft handelt, werden hier sicherlich auch keine Experimente in revolutionärer Resubjektivierung durchgeführt. Jedoch zeigt das Beispiel, dass es durchaus möglich ist, neoliberale Strukturen kreativ zu konfrontieren. Es geht hier um ein Manövrieren zwischen sich postfordistisch öffnenden Räumen und deren Neubesetzung durch neoliberale Fernsteuerung. Dabei ist ein hohes Maß an Pragmatik gefragt, denn neoliberale Macht funktioniert eben pragmatisch - nicht diskursiv.

\section{Schluss}

Wie diese Überlegungen zeigen, hat sich am Rande der Kreativindustrien ein bereits recht umfangreicher Kritikapparat konstituiert. Sein Einfluss auf die Politik und Praxis der Kreativindustrien muss jedoch wohl als eher gering eingeschätzt werden. Gegebenenfalls bildet die britische Kritik eine positive Ausnahme, da aufgrund der außergewöhnlichen Wucht, mit der die gerade beginnende Wirtschaftskrise Großbritannien treffen wird, hier alternative Stimmen mehr Gehör finden könnten. Dafür würde auch die stark ökonomische Ausrichtung der englischen Kritik - Creative Industries als verkehrte Wirtschaftspolitik - sprechen, die möglicherweise eine größere Anschlussfähigkeit gewährleistet. Für den restlichen Globus wird das creative industries paradigm wohl noch einige Zeit auf der stadtpolitischen Agenda stehen.

In einer zumindest potentiell äußerst interessanten Position befinden sich die aktivistischen und akademischen Exponenten der kulturwissenschaftlich orientierten Kritik, wie man sie vor allem auf dem europäischen Kontinent antrifft. Diese machen nämlich selbst einen wichtigen Teil der Kreativindustrien aus, zumal die Kritik auf hippen Konferenzen oder in angesagten Kunstgalerien inszeniert wird. Man kann in diesem Zusammenhang von einer so genannten immanenten Kritik - also einer Kritik aus der Praxis heraus - sprechen. Allerdings bleibt diese fragwürdig, solange sie in eine Anklage der Selbstunterwerfung kreativer Arbeiter mündet.

Ironischerweise zeigt sich in derartigen Bewusstseinsappellen gerade die fehlende Reflexivität der intellektuellen Dekonstrukteure, für die ja selber nicht weniger auf dem Spiele steht, als die Legitimation ihres eigenen prekären Arbeitsplatzes im intellektuellen Segment des Kreativsektors. KritikerInnen sind also mit dem Problem konfrontiert, sich ent- 
scheiden zu müssen zwischen Kritik als persönlicher Arbeitsbeschaffungsmassnahme und Kritik als materialer Intervention. Für erstere sind Dekonstruktionen der herrschenden Verhältnisse weiterhin ausreichend. Entscheidet man sich für die zweite Variante, wird es viel schwieriger: Dann geht es um die bereits erwähnte Frage von „subversivem“ Networking zum Zwecke der Wiedergewinnung von Verhandlungsmacht auf Seiten der verstreuten Kreativarbeiter. Dies ruft, vielleicht etwas überraschend, einen bekannten Kritiker des 19. Jahrhunderts auf den Plan, der gerne mit den Worten zitiert wird, es käme nicht darauf an, die Welt zu interpretieren, sondern darauf, sie zu verändern.

\section{Literatur}

BERR (2008): Manufacturing: New Challenges, New Opportunities, BERR (Department for Business, Enterprise \& Regulatory Reform). www.berr.gov.uk (Zugriff am 1.3.2009)

Bröckling, Ulrich (2003): „Über Kreativität. Ein Brainstorming“. In: Bröckling, Ulrich/Paul, Axel T./Kaufmann, Stefan (Hg.) Vernunft Entwicklung - Leben. Schlüsselbegriffe der Moderne. Festschrift für Wolfgang Eßbach, München: Fink, S. 235-243.

DCMS (Department of Culture, Media and Sport) (1998/2001): Creative Industry Task Force: Mapping Document, London.

Elliott, Larry/Atkinson, Dan (2007): Fantasy Island. Waking up to the Incredible Economic, Political and Social Illusions of the Blair Legacy, London: Constable \& Robinson.

Florida, Richard (2002): The Rise of the Creative Class. And How It's Transforming Work, Leisure, Community and Everyday Life, New York: Basic Books.

Foucault, Michel (2004): La Naissance de la Biopolitique. Cours au Collège de France 1978-1979, Paris: Gallimard/Seuil.

Friebe, Holm/Lobo, Sascha (2006): Wir nennen es Arbeit: die Digitale Bohème oder: Intelligentes Leben jenseits der Festanstellung, München: Heyne.

Harvey, David (2001): „The Art of Rent: Globalization and the Commodification of Culture“. In: Spaces of Capital: Towards a Critical Geography, New York: Routledge, S. 394-411.

Head, Simon (2003): The New Ruthless Economy. Work and Power in the Digital Age, Oxford: Oxford University Press.

Heartfield, James (2005): The Creativity Gap, www.design4design.com/creative.pdf (Zugriff am 15.1.2009) 
Hesmondhalgh, David/Pratt, Andy (2005): „Cultural Industries and Cultural Policy“. In: International Journal of Cultural Policy, 11 (1), S. $1-14$.

Holmes, Brian (2007): „Disconnecting the Dots of the Research Triangle: Corporatisation, Flexibilisation and Militarisation in the Creative Industries“. In: Lovink, Geert/Rossiter, Ned (Hg.): MyCreativity Reader: A Critique of the Creative Industries, S. 177-190.

Kolinko (2002): Hotlines, Call Centre, Inquiry, Communism, www.prol-position.net (Zugriff am 15.1.2009)

Lee, Terence (2004): „Creative Shifts and Directions. Cultural Policy in Singapore“. In: International Journal of Cultural Policy, 10 (3), S. 281-299.

Leunig, Tim/Swaffield, James (2008): Cities Unlimited: Making Urban Regeneration Work, www.policyexchange.org.uk (Zugriff am 15.1.2009)

Michale, Keane (2007): „Re-Imagining Chinese Creativity. The Rise of a Super-Sign“. In: Lovink, Geert/Rossiter, Ned (Hg.) MyCreativity Reader: A Critique of the Creative Industries, S. 99-109.

Malanga, Steven (2004): „The Curse of the Creative Class“. City Journal, Winter, S. 36-45. www.opinionjournal.com (Zugriff am 15.1.2009)

Manske, Alexandra (2008): „Kreative Superstars. Die soziale Platzierungsstrategie der Digitalen Bohème“. Polar. Zeitschrift für Politik. Theorie. Alltag, Märzausgabe. Frankfurt/Main: Suhrkamp S. 123126.

Marcuse, Peter (2003): „Review of ,The Rise of the Creative Class“ by Richard Florida“. In: Urban Land 62, S. 40-41.

Mommaas, Hans (2004): „Cultural Clusters and the Post-industrial City: Towards the Remapping of Urban Cultural Policy“. Urban Studies, 41(3), S. 507-532.

Osten, Marion von (2003): „Be Creative! Der Kreative Imperativ. Anleitung“. In: Norm der Abweichung, Zürich, Wien: Springer, S. 161165.

Oudenampsen, Merijn (2007): „Back to the Future of the Creative City: An Archaeological Approach to Amsterdam's Redevelopment“. In: Lovink, Geert/Rossiter, Ned (Hg.) MyCreativity Reader: A Critique of the Creative Industries, S. 165-176.

Pasquinelli, Matteo (2007): „ICW - Immaterial Civil War: Prototypes of Conflict within Cognitive Capitalism“. In: Lovink, Geert/Rossiter, Ned (Hg.) MyCreativity Reader: A Critique of the Creative Industries, S. 69-80. 
Negri, Antonio (1991): The Savage Anomaly: The Power of Spinoza's Metaphysics and Politics, Minneapolis: University of Minnesota Press.

Peck, Jamie (2005): „Struggling with the Creative Class“. In: International Journal of Urban and Regional Research, 29 (4): S. 740-770.

Qidi, Wu (2006): „Creative Industries and Innovation in China“. In: International Journal of Cultural Studies, 9 (3), S. 263-266.

Raunig, Gerald (2007): „Kreativindustrie als Massenbetrug“, transform.eipcp.net (Zugriff am 15.1.2009)

Ross, Andrew (2007): „Nice Work if You Can Get It: The Mercurial Career of Creative Industries Policy“. In: Lovink, Geert and Rossiter, Ned (Hg.) MyCreativity Reader: A Critique of the Creative Industries, S. 17-40.

Rossiter, Ned (2006): Organized Networks: Media Theory, Creative Labour, New Institutions, Rotterdam: NAi Publishers.

Sawicki, David (2003): „Review of , The Rise of the Creative Class“ by Richard Florida“. In: Journal of the American Planning Association 69, S. 90-91.

Thrift, Nigel (2006): „Re-Inventing Invention: New Tendencies in Capitalist Commodification“. In: Economy and Society, 35 (2): S. 279306.

Tong, Su (2006): „Cultural Resources, Creative Industries and the Long Economy“. In: International Journal of Cultural Studies, 9 (3), S. 307-316.

Xiaoming, Zhang (2006): „From Institution to Industry: Reforms in Cultural Institutions in China“. In: International Journal of Cultural Studies, 9 (3), S. 297-306. 
Neue Arbeitsformen 



\section{Reflections on Precarious Work in the Cultural Sector}

\section{ANGELA MCRobBIE}

In my work I have been concerned with how culture and creativity have in effect in recent years been put to work not as leisure, or as consumption, as taste or as national identity, but rather as sources for work, employment and job creation. Of course people have for many years been employed in the arts and cultural sectors, but there has been a seismic shift since the mid 1990s. From being rather gentle backwaters of employment, these areas have come to the forefront of the 'new economy'. The paradoxes and the contradictions this gives rise to will be the subject of this article. One element of my argument concerns the illegibility of work in the creative and cultural sector. But this is functional illegibility. What have been in the past considered sites of relative freedom have become quite the opposite, fields for self-disciplining, but without losing that freedom, indeed the freedom is harnessed and put to work, the new 'free' labour depends on this being entirely different from 'wage labour'. Hence my own designation of this as 'passionate work'.

Creative work appears to operate, as it did in the past, if only for the select few, as an escape from, the dull monotony of normal work. But here is another irony, culture has been prised out of the hands of those for whom it once had a compensatory existence (culture as a way of life) outside the workplace, for example in leisure, in the working men's choir, in the amateur singalong in the pub, in knitting circles, and has become instead a favoured instrument of self-help in regard to selfemployment, career choices, and small-scale entrepreneurship for those same people for whom it was external to work, for whom it was leisure (it has become culture as a way of making a living). Of course there 
have been jobs in the cultural sector, especially in the arts and in publishing and journalism, this has been a field typically associated with graduates in the arts, humanities and social sciences.

And the art school has embodied the tradition of State-supported training and educating for the industrialisation of art, design and more generally for the proliferation of culture within the nation-state. But now this expanded occupational terrain has become a site for a different kind of work, for working class and lower middle class young men and women, with or without degree qualifications. I am concerned then with what we might call a culture-machine, and with the deployment of creativity as a way of transforming what we mean by work, what it is to work. The promotion of creative work has I claim become a depoliticising strategy, a way of removing politics from work and replaceing it instead with notions of self-gratification, reward, and selfexpression.

First I will review some of the research I have carried out from the mid 1990s on the indie fashion sector, and then with young people working across a range of creative small scale organisations. Then I will update this work, drawing on some more recent interviews, in the third section I will offer a series of grounded commentaries a result of these more contemporary observations, and then finally I will expand the argument so that it connects with some of our concerns in the universities and the arts schools.

\section{Work as a mode of contemporary self-disciplining}

I have argued that passionate attachment to work has emerged as a mode of contemporary self-disciplining. My focus has been on fashion, but the same sensibility applies across the world of the arts, culture and media. I have drawn on Donzelot's brief article 'Pleasure in Work' to present an account of the incitement to take self-actualising pleasure in one's working life in fashion, as an example of how biopower operates as labour discipline for young women now entering the world of work with very different expectations than those of previous generations of women (Donzelot 1991). In effect this is a Foucauldian technology of the self, the subject is expected if not required to find pleasure in the working life. For young women working in new culture industry jobs, the valency of emotion and the experience of intensity in the new postFordist creative workplace, along with the existence of hidden hierarchies in seemingly flat working environments, and the absence of access 
to rights, and the further side-effect of flexibility as 'gender retraditionalisation', produce a kind of multi-layered opacity, or 'illegible labour' that is also a defining feature in this sector. There are three key themes that have emerged in the small studies of creative microeconomies that I have carried out in recent years (McRobbie 1989/1994, 1998, 2002).

The first feature is that fashion design activity, when pursued as a specialism, is low capital return work, by which I mean that there is an inversion of Beck's description of 'capital without jobs', such that these young women are working in a context of jobs without capital (Beck 2000). They had little or no investment capital apart from small bank loans, or support from the Princes Trust. As micro-enterprises or freelance outfits, their capacity to manufacture clothes for example, was limited, and this was in effect an integral part of their working practices. They would typically produce a collection of fifteen pieces and then manufacture runs of perhaps just 20 of each item.

Over time this style of independent working almost inevitably became unsustainable and instead had to become a stepping stone into more diverse activities. In the search for security the young women fashion designers gravitated to a public sector niche of a part-time or fractional appointment, ideally teaching, the advantage of this being that payment would be guaranteed and there might even be some statutory obligations on the part of the employers such as sick pay. This then became the most reliable of the roles tucked into the portfolio. Jobs like these were considered gold dust, and there was also a hierarchy in this kind of work, the best jobs being in prestigious art schools or universities, and the less attractive being in low level further education courses in fashion retail or perhaps in fashion buying.

This shift towards the public sector coincided however with the semi-privatisation of many of its activities in the world of the arts and culture. By the time the young women had decided to become mentors or arts administrators, these kinds of posts had already been stripped of the welfare benefits accruing to employment status and so were subject to not dissimilar insecurity as was experienced in the commercial world of project-working. There was something of an irony here. Thirty years ago, graduating from art school with a specialism in fashion and textiles for many young women would have been followed by a Post Graduate Certificate in Education course which in turn would have resulted in a teaching post in a secondary school, with all the benefits associated with the teaching profession, holidays, salary scales, and opportunities for promotion, including also the chance to keep professional skills alive with shows and exhibitions. 
Overall this would have meant being a teacher, which has never carried quite the status and glamour of actually being a fashion designer. Nowadays we might find that similarly qualified young women would pursue this independent, freelance pathway for many years, scraping a living, never thinking about undertaking a teacher training course since schoolteaching carries no aura of success, only to find themselves eventually in their late 30 s or early 40 s in effect working as teachers on 'projects' with unemployed youngsters or with young people, suspended from school or who have been forced onto job creation schemes. The women retain the title of fashion designer rather than that of fashion and textiles teacher, even though the bulk of their livelihood will actually come from this kind of teaching-mentoring activity. ${ }^{1}$ Opacity or illegibility creeps into these working lives, they have a semi-detached relation to the institutions where they have a seasonal teaching contract, and to keep themselves buoyant on the market they constantly need to be promoting their own work no matter how little time they have to concentrate on this creative activity, it is nevertheless still their trademark, giving them access to possible contracts and so it has to be safeguarded and updated even though in reality its existence might be almost virtual or imaginary

The second theme is then the unviability over time of a pure fashion or design specialism and the need, as a result, for a broad portfolio, hence the rise of the fashion multi-taskers who can show themselves capable of working in a number of different roles across this field. This might mean helping out as a shop assistant, developing new projects which emerge from perceived needs on the part of the sector and which connect directly with the knowledge economy ${ }^{2}$, picking up the skills of being a stylist, doing agency work booking photographers, compiling and then editing fashion directories, or working as a freelance design assistant or consultant for various less well-known or less adventurous companies such as Esprit or Marco Polo.

The interviews I conducted showed that the ingenuity and emphasis on contacts and networks needed to keep these jobs coming in, meant that unfairness, gender or race related discrimination, or overtly bad

1 The same applies to the category of "film-maker" who in reality might be teaching media production several days a week to young people who have been excluded from school, or who are on welfare to work programmes.

2 Harriet Posner for example began as an independent designer but some years later helped to set up a fashion directory, for use by designers and wholesalers as well as retailers who needed up to date information about suppliers and manufacturing units and costings across the world, see www.harrietposner.com. 
practice in the sector was often ignored and put down to experience. Most often the strategy was to walk away from it, having already spent energy and anger in situations where the payment had not arrived months after the job was completed, or the organisation had changed the terms mid-way through the work. The fashion world has long been known to operate in these ways, so there was some level of tolerance of bad practice. Gender inequalities were often inflected in a post-feminist way, for example women bosses treating young men more respectfully than their female counterparts, giving them more work as freelancers, or more complicated and hence better paid work, and so within this landscape of daily stress these gender issues were often difficult to pin down, they would be buried deep inside this whole underbelly of freelance experience. Work-place grievances would not be opened up for discussion, never mind arbitration, they simply meant it was time to leave, usually under a bad cloud of claims and counter-claims. Sociologists of small businesses that rely on freelance or casual workers would surely say there is nothing at all new here, and they are probably right. The novelty applies more to the sector itself, as art and design, culture and creativity take on the cruel characteristics (or bad intensity) of the world of small enterprises

The third feature can be described as a bypassing mechanism, which impacts on the career trajectories and self-identities in this sector. By this I mean the way in which the structural characteristics of this whole field of new culture industry working are increasingly predicated on mechanisms which allow the usual safeguards against poor practice or discrimination in the workplace to be by-passed and ignored without recrimination. Whether this gives rise to low level criminality or corruption or not, this possibility is normalised in the everyday working lives of the young people. The very idea of challenging a long hours culture is replaced again by emotional intensity, by profound attachments, by virtually living the work, by eating if not sleeping with the project director. There are also incentives to young cultural entrepreneurs to create flexible and informal working environments for themselves so that employment law is irrelevant. Even if in the course of time new legislation is indeed introduced to protect part-time or casual workers, in the meantime informal working and network sociality comprises a de facto understanding that there can be no recourse to the law or to an industrial tribunal when things go wrong.

By-passing the world of what Ulrich Beck calls 'normal work' means that the new cultural capitalism can get away with murder, since the kind of dangers which appear in old factory-style environments are replaced here by health hazards of a different kind, mental illness, burn 
out, exhaustion, alcoholism, drug-related problems, heart attacks, premature strokes and so on. It also results in what Scott Lash and then Lisa Adkins call gender re-traditionalisation in the form of a renewed dependency on family or kin as this becomes once again a unit of production (Beck/Giddens/Lash 1994; Adkins 2002). Lash commented that women were, in the world of reflexive modernisation 'reflexivity losers'. I think what this means is not just that old patterns of gender hierarchy re-appear in the form of the husband in what is in effect a family business both being more active in network sociality at night or in the evenings that must be spent in the pub, but that relations of financial dependence take on a new significance, that some work simply cannot be sustained without the support of a partner.

If new culture industry work coincides with the state attempting to unburden itself of responsibility through promoting self-employment in this sector, almost inevitably responsibility falls back on family and kin. It has long been the case that a struggling artist or writer has been dependent on his wife's steady employment to cover the basic bills and the mortgage, but when young women are embarking on these precarious careers and when it is quite uncertain as to whether or not they will actually secure a viable income, the fact that the state has cut back on benefits and on many other forms of social security, so that unemployment payments barely exist, and when so many creative-related jobs no longer provide security of employment, this introduces a retrograde factor for young women who not only want to 'be creative' but who also hope to be financially independent.

The chances are that they actually, over time, become less creative because the desire or the need for a steady income prevails. Bearing in mind that for this generational cohort of young women this is a priority, then it is likely that artistic independence and creative freedom are jettisoned in favour, eventually, of some form of regular employment, all the more so if the same women embark on motherhood ${ }^{3}$. Multi-tasking, project work, and job mobility as well as different forms of self-employment all contribute to this work becoming illegible labour. Statistics are unreliable, despite all the recent attempts by government to 'map' the new culture industries for the reason that the culture of creative work defies the logic of systematicity. Working life is thoroughly individualised and occupational identity has been subjected to the logic of branding, in direct contrast to the old world of bureaucracy and replaceability,

3 Destination studies have not so far investigated gender-specific pathways see Royal College of Art 2004. 
and instead uniqueness is a dominating characteristic, and the lifebiography the most reliable tool for charting pathways and forms of work and experience across this sector.

\section{Researching self-reflexive subjects}

Indeed we also come up against difficulties when we turn to the realm of experience and the research methods relied upon to document that experience. There is no (nor should there be) transparency of meaning in the narrative accounts given by these subjects, for the reason that, as Lange points out, they are generally highly self-reflexive subjects for whom the chance to express themselves in an interview situation or in the form of a research- related blog or email diary becomes indistinguishable from the self-promotional strategies required to positions themselves on the freelance labour market (Lange 2005). University research has a place in the cognitive map of the small scale cultural entrepreneur. This aspect of creative labour, the endless need to self-promote, and to flag up the work itself, makes it difficult for the researcher to disentangle the layers of talk which are required features of network sociality and which frequently overtake the flow of the well-planned semistructured interview. Nobody can afford to come across as less than exceptional, hard working, and talented. Bauman described the way in which failure is personalised and how self-blame and a must try harder attitude takes the place of more traditional workplace grievances (Bauman 1990). We can really only surmise that this kind of work takes its toll on mental health and well-being, and the question arises as to how people retain visibility and how and when and why they slip into the invisibility of quiet mode.

I offer a selection of quotes from three respondents. One is from the early work on fashion designers and is a simple expression of creative identity. It is by Pam Hogg, who in many ways epitomised the first wave of visible, successful, subcultural, small-scale art-related designers. I am interested in her career because, a forerunner of Galliano and younger by a decade than Westwood, she has refused the lure of the big commercial marketplace, while nevertheless finding it impossible to remain small scale and independent. For this reason over the last few years she has had a low profile, known only to insiders. (This reflects the point above about 'failure' and what it means, fading into invisibility, making a living by different means?) In fact Hogg has continued to make one off dresses and costumes for musicians and celebrities, she herself plays in a band, and is well known around the club scene in London. And for the 
first time in many years she is showing a new collection at London Fashion Week 2009 20th-27th Feb). I should also add that I know Pam personally from her time at the Glasgow School of Art. Her career is totally idiosyncratic, haphazard and irregular, archetypal artist-bohemian.

Sometimes I dream my next collection. I have millions of clothes in my head, that I have not got time to produce. It's like painting with the body as a canvas. I cut straight into the fabric, the ideas pour right out of me onto the table, I get completely carried away by the process. I like being able to sell clothes that are within the range of girls and young women, I get such a buzz seeing them wear my pieces out and about on the streets and in the clubs.

In total contrast and following up the idea of self-reflexivity and creative identity, one more recent couple of designers, recently graduated in graphic design, and working together in as partners, approached me, in the university, in the same way as they did potential clients. Their distinctive business strategy was to send out unusual and beautifully designed postcards to people who they thought they would like to work for or with.

When I responded to their postcard, and told them about my culture industry research, they were happy to take part in what turned out to be a lengthy interview. They had read my fashion book while at college and had found it interesting, they were also familiar with the Subcultures Reader, so in effect my research respondents came to me in this case, and three years after the interview they continue to send me postcards and emails. I in turn monitor their activity, which reveals them to be continuing to work at a frenetic level and keeping to the same kind of business plan they described to me in our conversations. The two hours I spent with them were illuminating.

They worked from home to keep down costs. They arranged to meet potential clients and to show them their portfolio and ideas in different London locations, in my own case one of the large and empty cafés in the South Bank. However what I hoped would be a research interview was in effect them presenting their portfolio to me and talking their way through it, every single client and job was described in detail, myself and my research assistant barely got a word in, such that asking questions got pushed off the agenda. This was of courser immensely interesting and research wise we just went with the flow.

They were also pleased to describe their daily activities and their working life since leaving college. Working from home, getting on the dole until contract began to flow in. Never borrowing or taking out loans, keeping overheard low. Never more than three projects at a time, 
so they could guarantee quality. Talking between themselves for days at a time on how to develop an idea. In effect they were offering a full design service to possible clients but on an out-sourced and one- off freelance basis. Researching in advance, they also provided clients with socially informed creative ideas about their products. Referring to their pitch with a key company, the young man, Mike commented:

After we had presented it, it was the accountants who said 'I can really see the benefit of this creativity', which was really nice.

These two young people, Rebecca and Mike, talked in detail about the technicalities of their work, the style, the colours, the 'linear sequences' and so on. My role in this research interview was more reminiscent of being a tutor walking about a graphic design studio and commenting on the work. But outcome tells us more about the work process than might be imagined. Enthusiasm, drive, and network sociality were the defining features of this kind of creative entrepreneurship.

The interview was an opportunity. Myself and the research assistant heard about every client they had worked for in the last 3 years, what they had done, what the outcome was, how they got the work, and what it led to next. We were also able to see the links between the client range, from Levi's to Diesel, from MTV to Cadbury's chocolate, from Dunhill to Channel 4.

My impression was that these companies were not looking to bring the couple in as part of a big advertising team but instead were willing to pay them for ideas, input and more specifically for a 'pure art' signature brought to the world of commercial design. The pair were working with clients not unlike the way in which as students they would be doing work or coming up with ideas for similar clients, but within the framework of 'work experience'.

This way of pitching gave them a foothold in a competitive market, and this time, unlike as students, they were being paid. Their strategy also showed the way in which art and design school pedagogy (internships, working unpaid for clients as part of the training) relayed into the world of freelance work. 'rebeccaandmike' (their trade name) came across as exemplary professionals whose creative identity was forged through bringing art and design, subculture and cultural studies, mediated through their training at Central St Martins, into the commercial domain of mainstream clients (they suggested Cadbury's did a special edition chocolate bar).

Last I offer just one quote from Paul Hedges director of the now very successful Hales Gallery in London (Bethnal Green). A Goldsmiths 
graduate and one of the Hirst generation, but with a sharper concern for social issues including local community politics, Hedges realised the combination of punk DIY ethos with the enterprise culture ideal espoused by the Thatcher generation. Hedges's career demonstrates the power of network sociality underpinned by the long hours, no pay culture. His first shows were for Jake and Dinos Chapman and then for Mike Nelson, and this would repay him on the longer term. To start up he raised $£ 85,000$ as a business training for artists initiative in the rundown area of Deptford where he had grown up, he used his connections with the local church to get cheap premises and he opened a café with space for a makeshift gallery. Talking to him about his career he said:

I was getting up at 5 am and making a load of sandwiches, and then by 3 in the afternoon I would get out of the kitchen and do the paperwork for the gallery.

What these extracts illustrate is the utterly singular and non-comparable nature of careers in this sector, and the uniqueness of life-biographies (although all trained in the UK art school system, and all identified themselves with aspects of youth culture as a kind of unofficial training ground for later careers).

I would suggest that conventional methodologies for carrying out social research on or with the creative workforce are less effective than research strategies which foreground life-biography. Cultural producers are comfortable talking about 'the work itself'. But as a cohort they are typically de-territorialised, nomadic, and rhizomatic in their schedules. Fleeting from project to project, the quality of social encounters is both intense and often short lived, by necessity. The economic geographer of cultural labour markets Allen Scott gets round the difficulties I describe above in relation to representativeness by using professional organisations as the source for respondents across the areas of film, media, TV and associated fields (Scott 2000).

While this gives methodological rigour and statistical accuracy, nevertheless there is little correspondence between these definable skills and competences and the working practices of the four people quoted above. A professional organisation which would represent their distinct interests was not something that had any place in the working lives of these people. Their own personal networks replaced this more traditional form of work organisation. Of course one might add that artists and creative people have in the past worked by and large without trade unions or professional associations, but the question arises that as this kind of work becomes increasingly pervasive with larger numbers of people amassing 
together in albeit fragmented or even virtual ways, the consolidation of networks into support systems might seem like an obvious thing to do.

\section{Expanding our understanding of creative labour}

In previous articles I have introduced a range of conceptual devices for expanding our understanding of this field of creative labour e.g. 'happiness at work', 'passionate work', 'club to company' 'culture as a way of life (Williams) to culture as a way of making a living', I have also discussed the visibility of $1 \mathrm{st}, 2$ nd and $3 \mathrm{rd}$ waves of creative economies in the UK and in the light of the extensive neo-liberalisation effect in global cities like London I have suggested the emergence of the 'one big hit' model or aspiration (McRobbie 2005). In the following pages I briefly want to address issues, which connect with the changing prevailing socio-economic structures which impact on this creative activity. This also takes us away from expressions of passionate attachment to work to the other side, and to the power relations, which produce intensity, and a highly emotional relation to work. For the sake of brevity and clarity I will itemise these as follows;

- The expansion of this kind of work as a feature of government policy which traverses all levels of schooling and education (e.g. media, performing arts, art and design etc.) marks a decisive feature for the labour market of the future in the UK and also Europe, in regard to unburdening companies, firms and conventional employers of the responsibility they have hard to workforce. It is one thing to talk about unburdening, but something quite different to examine how this actually happens on the ground.

- The training and education for self-organised work, results in a field of semi-employment which is relatively cheap to introduce as a kind of informal thin-spread labour strategy. And it provides labour, which comes cheap to employers as a result of its unofficial or irregular status. In effect irregular work with the shimmer of creativity becomes normalised and institutionalised according to the emerging codes of the de-regulated labour market.

- This has repercussions beyond the limits of creative labour for the reason that as we have seen this kind of work is profoundly intersectional, it overlaps with and becomes inextricably linked with parts of the knowledge economy, with the social projects for creating employment in Europe, as well as for implementing grounded social policies, in fact we could say there is a multi-chain model in place. To give one example, one layer of young people (i.e. under $35 \mathrm{~s}$ ) in Italy, with qualifications in areas as diverse as architecture, media 
and communications, social science, and law, are employed on a freelance basis, by an independent research organisation (whose directors are social scientists funded through an array of EU projects), to administer and oversee further down the chain, a scheme to provide skills and training to unemployed graduates in Palermo who might benefit from business studies, media education, tourism and heritage studies, and knowledge of enterprise culture to develop a more autonomous self-reliant outlook, so as to be able eventually to create their own jobs ${ }^{4}$. The conclusion of the funded project entailed the Palermo students getting work experience as interns in the creative sector in Berlin for up to four weeks (events management, youth employment, club management, fashion sector etc.). In this context regular employment is replaced by irregular, abnormal and illegible labour where they question of who and within what terms and conditions is paying who, who is on work experience or internships, and at what level of authority or 'status', disappears in a fog of activity, events, invoices and claims. This is 'social enterprise' drawing on cultural and creative skills and expertise, it offers a fascinating glimpse of how complex and inter-generational such EU funded projects are ${ }^{5}$.

- In small-scale or tiny organisations intimacy or emotional labour is unavoidable. To cut overheads people work in close proximity with each other, if hierarchies are flattened and bureaucracy marginalised, pseudo-kinship or familial ties replace workplace formality, and in such a context anger and even violent emotion replaces the grievance procedures in formal employment. Small companies have always been characterised by such volatile and intimate environments (the small restaurant, the small fashion company) however there are consequences when these kinds of features come to dominate organisations which technically are state- funded eg arts administration or semi-privatised training agencies.

- This leads us to reflect on the nature and status of workplace legislation, employment law, sex discrimination law, health and safety, sickness entitlement and maternity rights. In effect the field of employment rights comes into question. Are these evaded or simply ir-

4 My own contribution to this Palermo Step programme took the form of one lecture to the students followed by a seminar, and another lecture to a range of trainers.

5 Palermo Step Programme (2009) Sculoa Territoriale per Artisti Emergenti www.bbjshare.it. The Final Report will be published in May 2009. 
relevant, or are there new ways in which they are got round, or implemented in an unsystematic way?

- Finally however we need to consider business failure, this too is a site of illegibility. Especially when jobs are constantly being invented and re-invented, and where freelance work and project to project work dominates. To make sense of business failure or closure, we would also need to take into account not-for-profit dimensions, and the role of the third sector in so many cultural and creative sectors, e.g. film, media training for unemployed youth, or drama and music workshops for disadvantaged people, or community activities with a cultural and creative aspect, all of which are reliant on people inside the organisations re-applying for funding. What does it mean then when instead of talking about business failure the usual terms are that the organisation 'lost its funding'. Failure is then taken out of the working vocabularies and instead there are words like change, transformation or re-structuring. This makes it hard to see who are the winner and the losers. Or who eventually gets some compensation or pay out, who leaves amicable and who seeks recourse in law. I would suggest this area of illegibility is the source of acute anxiety, where roles are ill defined, where job descriptions evolve, where everyone is always waiting for the outcome of funding proposals. It is this dimension which accentuates the difference between regular and irregular work. It is this which leaves the individual unprotected, where the singularity, distinctiveness and uniqueness of the talented creative is a poor substitute for bureaucracy, and for that fact that if ill, one gets paid and someone can step in as a replacement.

\section{Conclusion}

We are confronted with a series of paradoxes, clusters of young creatives who pass on work opportunities to each other, yet also highly atomised and even isolated workers, for whom there are only a patchwork of possibilities to fall back on if things go wrong. As Allen J. Scott would put it they are both agglomerated and individualised, and also in cut throat competition for work, undercutting their peers when it comes to getting a project contract. Scott also quotes Ursell's description of an 'economy of favours' in the informal job market, but this too is hard to quantify, who is actually doing what for whom? There are other paradoxes, the state steps back, yet it re-appears in certain instances and in particular forms, employment law is swept aside in micro-organisations yet budgets are often underwritten by institutions of the state. The free- 
dom of creative labour is offset by traditional forms of dependency, family or kinship, husbands and wives. The visibility index of success overshadows the existence of failure or hardship, this is made invisible, people simply fade from the scene. To sum up, I have argued that creativity has been instrumentalised as a regime of freedom, bringing with it the possibility of happiness at work and the idea of culturally improved or enriched cities and environments, and this becomes the means by which older bureaucratic safeguards and entitlements are swept away. The theorists of immaterial labour correctly see this process as a manifestation of capitalist power and domination, as work subsumes life and as post-fordist production appears to respond to the desires of the people who fought for freedom from wage labour and from monotonous and mundane work. Cultural capitalism shows itself re-invigorated through its ability to absorb and respond to critique. Some theorists claim that post-fordism is capitalism's communism. My own conclusions are more banal:

- There are more creative producers than before and the numbers are rising as government invests in training more young people for this field of work (for example in Further Education colleges with media and performing arts, in projects for young offenders with film and media making projects), but this provision is predicated on uncertainty and unknown outcomes, since the capacity of the labour market to absorb this kind of work force is unsure, as is the sustainability of a DIY labour market concocted out of these self-realising strategies.

- We as educators and as people who both contribute to policy-making and implement it, find ourselves in an unexpectedly 'hot seat' position as universities and art schools are pinpointed as lead institutions for growth in the creative and the knowledge economy, the role of education and research in this area assumes a new importance. How do we ourselves in our pedagogy confront the uncritical celebratory discourse of the new cultural economy?

- If as the Italian Opera theorists claim work now overwhelms life itself we need to address the question of what new or novel modes of radical political organisation can be envisaged in such a context? When cultural and creative work in the community supplants socialwork we need to address the question of the immanent democratic politics of that illegible activity, which as Virno reminds us, rubs us so close to its opposite as to be frequently hard to discern.

To conclude I want to suggest we need to pay more analytical attention to not just the subsumption of life by work but to life itself, how does 
life intersect with these working practices, can everyday life be used as a possible instrument for critique against the overwhelming authority of work, can life be a source of creative opposition, and what might such a new form of everyday life look like in this context? I think we need to inspect more closely the fine line between paid and unpaid work, and focus in much more detail on what it might mean to re-invent a 'welfare state' in the light of the proliferation of precarious work.

\section{References}

Adkins, Lisa (2002): Revisions: Gender and Sexuality in Late Modernity, Buckinghamshire: The Open University Press.

Bauman, Zygmund (2000): The Individualized Society, Cambridge: Polity Press.

Beck, Ulrich (2000): The Brave New World of Work, Cambridge: Polity Press.

Beck, Ulrich/Lash, Scott/Urry, John: (1994) Reflexive Modernisation, Cambridge: Polity Press.

Donzelot, Jachques (1991): 'Pleasure in Work'. In: Burchell, Graham/Gordon, Colin/Miller, Peter (eds.): The Foucault Effect; Studies in Governmentality, Chicago: University of Chicago Press.

Lange, Bastian (2005): 'Landscapes of Cultural Scenes: Socio-spatial Emplacement Strategies of "Culturepreneurs" in Berlin'. In: d'Hauteserre, Anne-Marie/Terkenli, Theano S. (eds.): Landscapes of a New Cultural Economy of Space, Dordrecht: Kluwer Press, pp. 41-67.

McRobbie, Angela (1989/1994): 'Second Hand Dresses and the Role of the Rag Market'. In: McRobbie, Angela (ed.): Postmodernism and Popular Culture, London: Routledge, pp. 135-154.

McRobbie, Angela (1998): British Fashion Design: Rag Trade or Image Industry? London: Routledge.

McRobbie, Angela (2002): 'Club to Company'. In: Cultural Studies, Vol. 16, No. 4, pp. 516-532.

McRobbie, Angela (2005): The Los Angelisation of London. See: www.eipcp.net

Scott, Allen J. (2000): The Cultural Economy of Cities, London and New York: Sage. 



\section{Allein oder gemeinsam? Selbständigkeit in der Wiener Kreativwirtschaft}

HubERT EICHMANN, STEFAN LEITNER-SIDL

Unter Ausblendung diverser Schwierigkeiten der statistischen Erfassung sowie der inhaltlich begründeten Zuordnung von Berufsfeldern zur Kreativwirtschaft, sind in Wien entlang einer breiten Definition etwa 120.000 Personen oder $14 \%$ aller Erwerbstätigen in ,kreativwirtschaftlichen“ Sektoren tätig (Landsteiner/Harauer 2004). Etwa $30 \%$ davon arbeiten in den engeren Kunst- und Kultursektoren (darstellende und bildende Kunst, Musik usw.); dagegen $70 \%$ in den ,,angewandten“ Feldern: Architektur; Grafik, Mode, Design, Fotografie; Film, Rundfunk; Internet, Software; Werbung.

Das an sich bekannte Muster der Konzentration kreativwirtschaftlicher Milieus in Ballungsräumen ist in Österreich besonders ausgeprägt, da Wien mit ca. 1,8 Mio. Einwohnern die einzige Großstadtregion ist und insofern überregionale Sogwirkung ausübt (Bildungsinstitutionen, Absatzmärkte usw.). Dazu ergibt eine aktuelle Befragung unter Kunstschaffenden in Österreich, dass $44 \%$ (!) als Lebensmittelpunkt Wien anführen (Standard, 22.8.08, 29), während der Anteil von Wien an der Gesamtbevölkerung nur bei rund $20 \%$ liegt.

Zeitreihenanalysen für die Wiener Kreativwirtschaft in den Segmenten „content origination“ ergeben zudem einen signifikanten, wenn gleich konjunkturabhängigen Beschäftigungszuwachs (Mayerhofer/Huber 2005). Dabei ist die Dauerhaftigkeit („Nachhaltigkeit“) einmal geschaffener Arbeitsplätze in der Wiener Kreativwirtschaft höher als in der Wiener Gesamtwirtschaft: Während in allen Wirtschaftssektoren in Wien nach drei Jahren nur noch rund $38 \%$ aller neu geschaffenen Jobs existieren, liegt dieser Anteil in der Wiener Kreativwirtschaft immerhin 
bei $42 \%$. Strukturell lässt sich die Beschäftigungssituation in der Wiener Kreativwirtschaft durch eine kleinteilige Unternehmenslandschaft, einen starken Andrang von NeueinsteigerInnen (der die „Aufnahmekapazität" oft übersteigt) und (eben deshalb) einen hohen Anteil an selbständigen Beschäftigungsverhältnissen skizzieren.

Eine im Jahr 2005 durchgeführte Erhebung bei ca. 900 Erwerbstätigen in den ,angewandten“ Branchen der Wiener Kreativwirtschaft (laut obiger Definition) ergibt dazu das folgende Bild (Eichmann/Schiffbänker 2008): $13 \%$ der Befragten sind Arbeitgeber, $49 \%$ sind formal (Allein-)Selbständige (inkl. Freiberufler, Werkvertragnehmer usw.), 10 $\%$ arbeiten als „Patchworker“ (d. h. in Mehrfachbeschäftigungen) und nur $28 \%$ sind Angestellte (am ehesten in IT-Firmen, Werbeagenturen und beim ORF). Prägende Leitbilder sind eine professionelle, möglichst eigenverantwortliche Arbeitsausführung und/oder künstlerische Selbstverwirklichung. Dagegen werden Managementfunktionen oder eine Rolle als Unternehmer, d. h. als Arbeitgeber, nur von einer Minderheit angestrebt. Begleiterscheinungen dieser Kultur der Selbständigkeit sind ausgesprochen erwerbszentrierte Lebensmodelle sowie die Akzeptanz von eingeschränkter sozialer Sicherheit. Teilweise bescheidene Einkommen, intensiver Wettbewerb, oftmals geringe Honorierung der eigenen Leistung bzw. eine unsichere Auftragslage sind weitere Kehrseiten selbständiger Arbeit bzw. kreativ-künstlerischer Ambitionen.

\section{Förderungen und Interessenvertretung}

Als spezielle Initiative für die Wiener Kreativwirtschaft ist die von der Stadt Wien 2004 ins Leben gerufene Förderagentur Departure zu nennen, die sich auf Einzelprojekt- bzw. Unternehmensförderung in den Sparten Design, Multimedia, Musik und jüngst auch Architektur spezialisiert hat. Zwischen Mai 2004 und September 2008 wurden Fördermittel von insgesamt 10,7 Mio. $€$ vergeben, meist an Klein- und Kleinstbetriebe, wodurch ein privates Investment von ca. 43 Mio. $€$ ausgelöst wurde (www.departure.at).

Darüber hinaus ist die Förderlandschaft für Creative Industries in Wien bzw. Österreich vielfältig (was nicht mit üppiger Dotierung gleichzusetzen ist): Analog zur Ausdifferenzierung der kunst- und kulturpolitischen Felder bzw. der öffentlichen Institutionen existieren eigene „Schienen“ (und Konfliktlinien) für Filmwirtschaft, Architektur und besonders für die Hochkultursparten Musik, Theater, Museen usw. Nicht zuletzt deshalb, weil in Wien/Österreich die öffentliche Subventionierung der klassischen Hochkultur wesentlich großzügiger dotiert ist als 
jene für zeitgenössische Kunstformen und Kreativfelder, sind Vorschläge zur Umstellung von Subjektförderung (v. a. für Unternehmen) auf Objektförderung (Infrastrukturen, Plattformen, Netzwerke) etwas verfrüht. Die Ziele der laufenden Kreativwirtschaftsprogramme liegen ja gerade im Aufbau von international konkurrenzfähigen (und sichtbaren) Unternehmen bzw. von kritischen Massen zumindest in einigen Sparten.

Wichtig erscheint uns im Kontext von wirtschafts- (und auch arbeitsmarktpolitischen) Fördermaßnahmen für die Kreativwirtschaft das Hinterfragen der traditionellen Vorstellung, von der die österreichische Förderpolitik nach wie vor geleitet ist. Gleichsam in trauter sozialpartnerschaftlicher Einigkeit gehen Stadtpolitik, Arbeitgeber- und Arbeitnehmervertretungen davon aus, insbesondere solche ,,mittelständische" Unternehmen als förderungswürdig anzusehen, die eine mehr oder weniger ambitionierte Wachstumsstrategie behaupten. Unterstützt werden sollen „blühende“ Klein- und Mittelbetriebe, die den Standort sichern sollen, indem sie eine ausreichend hohe Zahl von stabilen und gleichzeitig innovativen Arbeitsplätzen generieren.

Im Allgemeinen ist an diesem förderpolitischen Leitbild wenig auszusetzen. Im Speziellen wird dadurch allerdings die Mehrheit aller Unternehmen (nicht nur) in der Kreativwirtschaft ausgeschlossen, d. h. Ein-Personen-Unternehmen (EPU) bzw. solche, die es auch in Zukunft bleiben wollen. Selbständige unterliegen insofern im sozialpartnerschaftlich verfassten Österreich - weiterhin - dem Risiko, interessenpolitisch „durch den Rost“ zu fallen. Insofern geht es zuallererst darum, die Existenzform Solo-Selbständigkeit anzuerkennen, anstatt diese als irrelevant oder atypisch zu marginalisieren.

Ein weiterer maßgeblicher Baustein zur Förderung von EPU (inkl. Werkvertragsnehmer usw.) wäre die rechtliche und materielle (Mindest-) Absicherung gegenüber Auftragslosigkeit = Arbeitslosigkeit. Die Einbeziehung von Selbständigen in eine Versicherung zum Schutz gegen Einkommensausfall bezieht sich selbstredend nicht nur auf die Kunst- und Kreativwirtschaft, sondern auf alle Branchen. Individuell abgeschlossene Verdienst- oder Betriebsausfallsversicherungen sind für jene mit dem dringendsten dahingehenden Bedarf meist nicht leistbar, und in den aktuellen Regelungen der österreichischen Künstlersozialversicherung sind lediglich marginale Leistungen für Kranken-, Unfall- und Pensionsversicherung enthalten, aber kein Schutz gegen Arbeitslosigkeit. Weitere Benachteiligungen von Solo-Selbständigen betreffen Regelungen zur Einkommenssteuer und Sozialversicherung oder wenig transparente Übergänge zwischen Beschäftigungsformen, z. B. in Bezug auf die Mitnahme ,angesparter“ Sozialversicherungszeiten (vgl. www.fofos.at). Freilich ist ein Wechsel zu einem allgemeinen Sozialversicherungssys- 
tem für alle Erwerbstätigen (wie z. B. in Schweden, vgl. dazu SchulzeBuschoff/Schmidt 2006) angesichts der Pfadabhängigkeit (und Trägheit) von Systemen wohl nur langfristig realisierbar.

Aufgrund dieser Auflistung sind allzu hohe Erwartungen auf der Makroebene eher unrealistisch: Wünsche nach besserer sozialer Absicherung stehen einer eingeschränkten Bereitschaft zur umfassenden Solidarversicherung gegenüber; Verbände bzw. Interessenvertretungen in der Kreativwirtschaft sind relativ schwach und/oder zersplittert. Naheliegendere Wege in Richtung ,nachhaltiger“ Arbeit sind daher arbeitsteilige Formen der Organisation auf der Mesoebene, die für Selbständige vor allem in flexiblen Kooperationen, Projektnetzwerken, Arbeits- oder Bürogemeinschaften usw. liegen. Ein derartiges Beispiel, dem zumindest in Wien eine Vorbildwirkung zukommt, wird im Anschluss skizziert.

\section{UnternehmerInnenzentren Schraubenfabrik, Hutfabrik, Rochuspark}

Im Jahr 2002 gründete die Firma Konnex (Michael Pöll/Stefan LeitnerSidl) in Wien Leopoldstadt eine Bürogemeinschaft in einer alten Schraubenfabrik. Als kleine Marktforschungs-Agentur war Konnex damals in der Situation von vielen Selbständigen und Kleinst-Unternehmen, die nach einiger Zeit im Home-Office das Bedürfnis verspüren, aus den privaten Räumen raus zu kommen und in Kontakt mit Gleichgesinnten zu treten. Das anfangs leere Fabrik-Loft in der Schraubenfabrik bot zudem die Möglichkeit, diesen Raum so zu gestalten und zu nutzen, wie es den eigenen Bedürfnissen entsprach. So wurde die notwendige Büroinfrastruktur installiert, dazu kamen Elemente wie Terrasse, Sofas, Kaffeebar, Tischfußball-Gerät, die dem ganzen Projekt eine soziale und spielerische Funktion verliehen.

Wände wurden nur für Archiv und Besprechungsraum gebaut, ansonsten blieb das Loft offen. In kurzer Zeit schlossen sich viele Selbständige, die zum Großteil in der Wiener Kreativwirtschafts-Szene angesiedelt waren, der Bürogemeinschaft an: Grafiker, PR-Agenten, Architekten, Eventmanager, IT-Firmen, Mode-Designer usw. Das Organisationsmodell war jedoch, anders als bei vielen kleinen Bürogemeinschaften, so gestaltet, dass Konnex für sämtliche Investitionen sowie die infrastrukturelle Betreuung verantwortlich war. Die Mieter zahlen dafür eine monatliche Miet- und Servicepauschale, in der sämtliche Bürokosten inkludiert sind. Innerhalb dieses Rahmens entstand in kurzer Zeit ein reges selbstgesteuertes Community-Leben. Feste, Betriebsausflug, 
Weihnachtsfeier, Vernissagen und vieles mehr wurde organisiert. Journalisten berichteten über das Konzept, den Ort und die Leute. Alsbald entstand eine Marke, die innerhalb der Wiener Kreativszene bekannt war. Spätestens nach einem Jahr merkten die Betreiber, dass sie hier nicht nur einen Eigenbedarf befriedigten, sondern ein Bedürfnis vieler Selbständiger nach Gemeinschaft, Austausch, Inspiration und einem passenden Ambiente. 2004 wurde eine weitere Etage der Schraubenfabrik gemietet und die Hutfabrik in Wien Mariahilf gegründet, 2007 der Rochuspark, eine ehemalige Schmiede in Wien Erdberg, aus der Taufe gehoben. Insgesamt umfasst die Selbständigen-Community im Jahr 2008 rund 80 Personen. Eine formelle Befragung in der Schraubenfabrik aus dem Jahr 2004 über die Motive der MieterInnen, in diese Bürogemeinschaft zu ziehen, brachte folgendes Ergebnis:

- Wichtigster Grund war, aus der sozialen Isolation des Home-Office bzw. des Einzelbüros zu entkommen und trotz der Selbständigkeit als Einzelne/r einer Gemeinschaft zugehörig zu sein.

- Gleich dahinter kam bei vielen die Perspektive, aus dieser Community auch ökonomischen Nutzen in Form von Aufträgen, Weiterempfehlungen, Projekt-Kooperationen usw. zu ziehen.

- Als dritt-wichtigstes Motiv wurde genannt, dass es für viele einen Wert darstellte, unter dem Dach einer mittlerweile bekannten Marke $\mathrm{zu}$ arbeiten. Beweis dafür ist, dass viele ihre Adressangaben auf Visitenkarten und E-Mail-Signatur mit den Worten ,,in der Schraubenfabrik" ergänzen.

- Erst an vierter Stelle der Motivpyramide kam die von den Betreibern ursprünglich intendierte Bedarfsbefriedigung hinsichtlich einer kostengünstigen und vollwertigen Arbeitsinfrastruktur.

Über die Jahre wurde nicht nur in diversen Medien über die geschaffene Community berichtet. Es interessierten sich zunehmend Wissenschaftler für das Konzept und zuletzt auch Politik und InteressensvertretungsVerbände. So kam es, dass die Konnex-Betreiber immer mehr auch als Experten für die Szene der Wiener Kreativwirtschaft gesehen und gefragt wurden und für ihre Kunden bei verschiedenen Anlässen auch eine informelle Interessenvertretungs- bzw. Lobbying-Funktion übernahmen. Die selbstgesteuerten Aktivitäten im beruflichen Bereich innerhalb der jeweiligen Bürogemeinschaften reichen von der Weiterempfehlung von Büro-KollegInnen, über die interne Beauftragung bis hin zu Projektkooperationen, sogar lose Firmenzusammenschlüsse waren schon zu beobachten. Auch informelle Einkaufsgemeinschaften wurden bereits gegründet (z. B. gegenüber einem Mobilfunk-Betreiber). Als Vision sehen die Betreiber eine Kooperation mit ähnlichen Initiativen in anderen Me- 
tropolen Europas, die einen informellen Austausch - physisch und virtuell - ermöglichen. Dies kann einen Schritt in Richtung Professionalisierung und Internationalisierung der in der Kreativwirtschaft Tätigen darstellen. Weitere Schritte sind der leichtere Zugang zu Kapital, v. a. Mikrokrediten, die Etablierung von einschlägigen Aus- und Weiterbildungs-Konzepten sowie der Gründung eines eigenen Mediums für diese spezielle Szene.

\section{Literatur}

Eichmann, Hubert/Schiffbänker Helene (Hg.) (2008): Nachhaltige Arbeit in der Wiener Kreativwirtschaft? Architektur, Design, Film, Internet, Werbung. Wien: LIT-Verlag.

Konnex (Hg.) (2008): People Spaces Action: Schraubenfabrik, Hutfabrik, Rochuspark. Wien: Eigenverlag.

Landsteiner, Günther/Harauer, Robert (2004): „Creative Industries in Wien - Methodologie und Befunde“. SWS-Rundschau 3/2004.

Mayerhofer, Peter/Huber, Peter (2005): Arbeitsplatzeffekte und Betriebsdynamiken in den Wiener „Creative Industries“, www.wu-wien.ac.at (Zugriff am 31.1.2009)

Schulze-Buschoff, Karin/Schmidt, Claudia (2006): „Allein, flexibel und mobil. Solo-Selbständigkeit nimmt in Europa stark zu“. WZWMitteilungen, Heft 112. 


\title{
„Neue Orte für neues Arbeiten"“: Co-working Spaces
}

\author{
BASTIAN LANGE, INGA WELLMANN
}

\section{Betahaus}

Werte werden nicht mehr in klassischen Büros geschaffen. Wertschöpfung findet statt in Projekten, an unterschiedlichen Orten, zu unterschiedlichen Zeiten, unabhängig, ohne Festanstellung. Diese neue Art der Arbeit findet an neuen realen und virtuellen Orten statt. Benötigt werden offene, digital vernetzte kollaborative Arbeitsorte, die flexibel sind und als Inkubationsplattform für Netzwerk, Innovation und Produktion dienen. Betahaus ist eigentlich eine Plattform, die den Ansprüchen von unabhängigen Kreativen und Wissensarbeitern gerecht wird. In einer Mischung aus entspannter KaffeehausAtmosphäre und konzentriertem Arbeitsumfeld legen wir Raum zwischen Arbeit und Privatsphäre an, in der kollaborative Innovation und Kreativität gefördert wird. (www.betahaus.de)

\section{Hallenprojekt}

In Anlehnung an den Begriff „Halle“ des Arbeitsphilosophen Frithjof Bergmann wollen wir Orte für Arbeitsformen der post-industriellen Gesellschaft schaffen. Wir wollen Orte für kreatives Arbeiten in so komfortabler wie inspirierender Atmosphäre schaffen, denen man wie einem Fitnessstudio gegen einen monatlichen Mitgliedsbeitrag beitreten kann, ohne sich sonst wie zu irgendwas zu verpflichten. Es soll Bereiche und Infrastrukturen für konzentriertes Arbeiten geben genauso wie Bereiche zur Entspannung und Zerstreuung,

1 Claim vom „Hallenprojekt“ (siehe: blog.hallenprojekt.de). 
Meeting- und Seminarräume genauso wie Cafébereiche, und all das rundherum auf analoge wie digitale Bedürfnisse der Neuzeit angepasst.

(wiki.hallenprojekt.de)

\section{Beehive Baltimore}

Beehive Baltimore is a community of freelancers, independents, entrepreneurs, and artists who want to augment the work-at-home lifestyle by working and participating in a community that's designed to support and encourage people who make a living doing what they love. (beehivebaltimore.org)

\section{Co-working Spaces}

Der Begriff Co-working heißt ,nebeneinander arbeiten“. Doch das wäre nur die eine Seite eines zunehmend um sich greifenden Phänomens. Coworking verbindet Arbeits- und Kommunikationsprozesse, in dem sich diese Praktiken nicht nur digital verorten, sondern ebenso an konkreten Arbeitsräumen. Erstmalig formierten sich Co-working Spaces durch lose Netzwerkbeziehungen von Computerfreaks. In dem sie sich mit ihren Computern vernetzten und Plattformen im Web aufbauten, verdichteten sie ihre Interaktionsprozesse. Temporären Meetings folgte der Gang in Cafés mit W-Lan Anschluss. Irgendwann kristallisierte sich die Idee heraus, dass man für diese Praxis ebenso eigene Räume definieren könnte: Die Idee war geboren, die Community existierte bereits und man zog mit seiner Kerngruppe in günstig zu mietende Fabriketagen.

Mittlerweile besetzen auch Akteure unter dem Vorzeichen Coworking das Feld, die als Dienstleister Räume aktivieren und den zahlreichen Mikrounternehmern der Kreativwirtschaft günstige und flexibel nutzbare Arbeitsräume anbieten. Sie verfolgen das Ziel, das Konzept des Co-working Space auch für andere Berufsgruppen und Personen in Angestelltenverhältnissen zu öffnen, um eine Flexibilisierung von Arbeitszeit und -raum zu ermöglichen und damit mehr Selbstbestimmung zuzulassen.

Als Phänomen entwickelt sich Co-working aber seit einigen Jahren vor allem in den USA, wo erste Pioniere dieser Bewegung die Open Source-Logik als freie Nutzung und Weiterverarbeitung eines Quellcodes auch auf andere Bereiche ausweiten wollten. Nicht nur Programme und Ideen sollten mit einer Community geteilt und dadurch öffentlich zugänglich werden, sondern auch Arbeitsorte. Inzwischen finden sich entsprechende Co-working Spaces bestens miteinander vernetzt und in unterschiedlichsten Ausprägungen über den gesamten Globus verteilt, vor allem aber in Metropolen. Co-working Spaces bestehen demzufolge 
aus thematisch fokussierten Communities respektive deren Arbeitsinfrastrukturen. Dazu gehören ein konkreter Arbeitsort und eine digitale Plattform. Der Ort kann flexibel auf die verschiedenen Projektarbeiten abgestimmt werden. Die Arbeitsplätze in Form eines gemieteten Arbeitstisches können kurzfristig und spontan angemietet werden. Die Räume weisen sich oftmals durch einen improvisierten Charakter aus.

Ihr flexibler Charakter korrespondiert mit dem Anspruch der Nutzer, einen freien Denkraum ebenso wie einen sozialen Raum zu haben, in dem Gespräche möglich sind und der gegebenenfalls auch Rückzugsorte bereithält. Präsentationsräume mit Café- und Lounge-Anschluss runden die kommunikativen Erwartungshaltungen in der Regel ab. Voneinander unabhängige und projektweise arbeitende Programmierer, Schriftsteller oder Designer teilen sich hier nicht nur gemeinsam eine Büroinfrastruktur, sondern unterstützen sich gegenseitig bei Bedarf auch mit branchenspezifischen Hilfeleistungen. Nebenbei entwickelt sich ein soziales wie berufliches Netz. Sebastian Sooth vom Hallenprojekt in Berlin:

Das Ganze ist eine Mischung aus Großraumbüro, W-Lan-Café und Home Office, und all das eben mit Leuten, die man nicht unbedingt vorher kennen muss, mit denen man auch nicht zwangsweise an einem gemeinsamen Projekt arbeiten muss, mit denen man aber trotzdem eine Ebene für gemeinsamen Austausch findet.

\section{Car-Sharing für Arbeitsplätze}

Ein wesentliches Merkmal von Co-working Spaces ist die lose Kopplung unterschiedlicher Raumprogramme, die sich an dem physischen Ort „Co-working Space“ berühren und direkt überschneiden. Es sind reale und gleichsam virtuelle Orte der Vergemeinschaftung von sich zunehmend professionalisierenden kreativen Wissensarbeitern. Der Charakter dieser Räume wird wesentlich durch die Operationspraktiken und Prozessverläufe der Wissensarbeiter bestimmt: Sie sind weniger auf Repräsentation ausgerichtet, vielmehr sollen sie aufgrund der flexiblen Nutzungsdauer sachlich und nüchtern drei Dinge bereitstellen: Strom, WLan und einen Sitzplatz. Sebastian Sooth, vom Hallenprojekt in Berlin:

Die Idee ist, eine Art Car-Sharing für Arbeitsplätze anzubieten. Die entsprechenden Orte können leerstehende Büroetagen in Städten sein, große Hallen, temporäre Architekturen zum Beispiel bei Festivals oder aber auch einfach nur ein Arbeitsplatz bei Max im Garten. Theoretisch könnte jeder seine eigene Umgebung zur Verfügung stellen. Hauptsache, man kann dort arbeiten. 


\section{Katalysator und Kompetenzpool}

Co-working Spaces sind nicht nur eine Reaktion auf kleinteilige und mikrounternehmerische Arbeitsformen in der Kreativwirtschaft. An diesen Verdichtungsräumen eröffnet sich ein basarartiger Tauschhandel mit immateriellen Gütern und individuellen Kompetenzen. Ein Steuerberater erhält für die Erstellung einer Jahressteuererklärung von einem Webdesigner die Erstellung seiner Visitenkarten im Web, während ein Innenarchitekt bei der Erstellung eines Auftrags mit einem Sounddesigner kooperiert. Co-working Spaces basieren zunächst auf Kulturen des Zufalls, des Spontanen, auf der Basis hochgradig vernetzter Akteure.

Die Verdichtung an einem Ort hat katalytische Wirkung und kreiert für alle Beteiligten einen strategischen Mehrwert: Die Heterogenität der individuellen Kompetenzen macht nicht nur Spass, sie generiert neue Formen der Innovation, die wir als Lowtech im Gegensatz zu den bekannten Hightech und finanzintensiven Innovationsbereichen (Bio-, Nano- oder Halbleitertechnologie) ansprechen. Der Begriff Lowtech bezieht sich auf ein technisches Verständnis, das sich unter den Maßstäben Funktion, Herstellung, Bedienung und Robustheit durch Einfachheit auszeichnet. Christoph Fahle und Tonia Welter vom Betahaus in Berlin:

Betahaus wird ein Kristallisationspunkt, er besteht zum einen aus freien Kreativen, die jeden Tag hier arbeiten und sehr starker Bestandteil des Kerns der Community sind, und anderen, die über die virtuelle Plattform verbunden sind und nur ein bis zwei Mal im Monat reinschauen. Dadurch lösen wir das Home Office auf und schaffen einen kontinuierlichen Austausch, der am Ende neue Ideen generiert.

\section{Sozialstatiker und Begegnungskuratoren}

Nun zu glauben, ohne Steuerung emergieren gleichsam an diesen Coworking Spaces spontan Innovationen, würde übersehen, dass es sich im Grunde genommen um kuratorisch inszenierte Orte handelt. Die Betreiber derartiger Co-working Spaces weisen sicher nicht durch rein altruistische Motive aus. Sie haben nur die richtige Idee $\mathrm{zu}$ einem richtigen Zeitpunkt entwickelt und diese in einen sich durch den ökonomischen Wandel transformierenden Stadtkörper platziert.

Die dadurch ausgelöste Flexibilität in der Nutzung der zunehmend porösen Stadtstrukturen (Leerstand) erfolgt von unten, und erst langsam erkennen Immobilienbesitzer die Chance, die Kreativen nicht nur als Trockenwohner zu instrumentalisieren, sondern dauerhafte Provisorien der postmodernen Stadtnutzung einzurichten. Dass die entsprechenden 
Orte durch den verstärkten „Traffic“ einer bestimmten Szene gleichzeitig auch sozial, kulturell und damit auch in ihrem monetären Wert aufgewertet werden, ist eine Begleiterscheinung, die gegebenenfalls auch zur Gentrifizierung führen kann.

Abbildung 7: Ausschnitt des Co-working Space Betahaus in Berlin

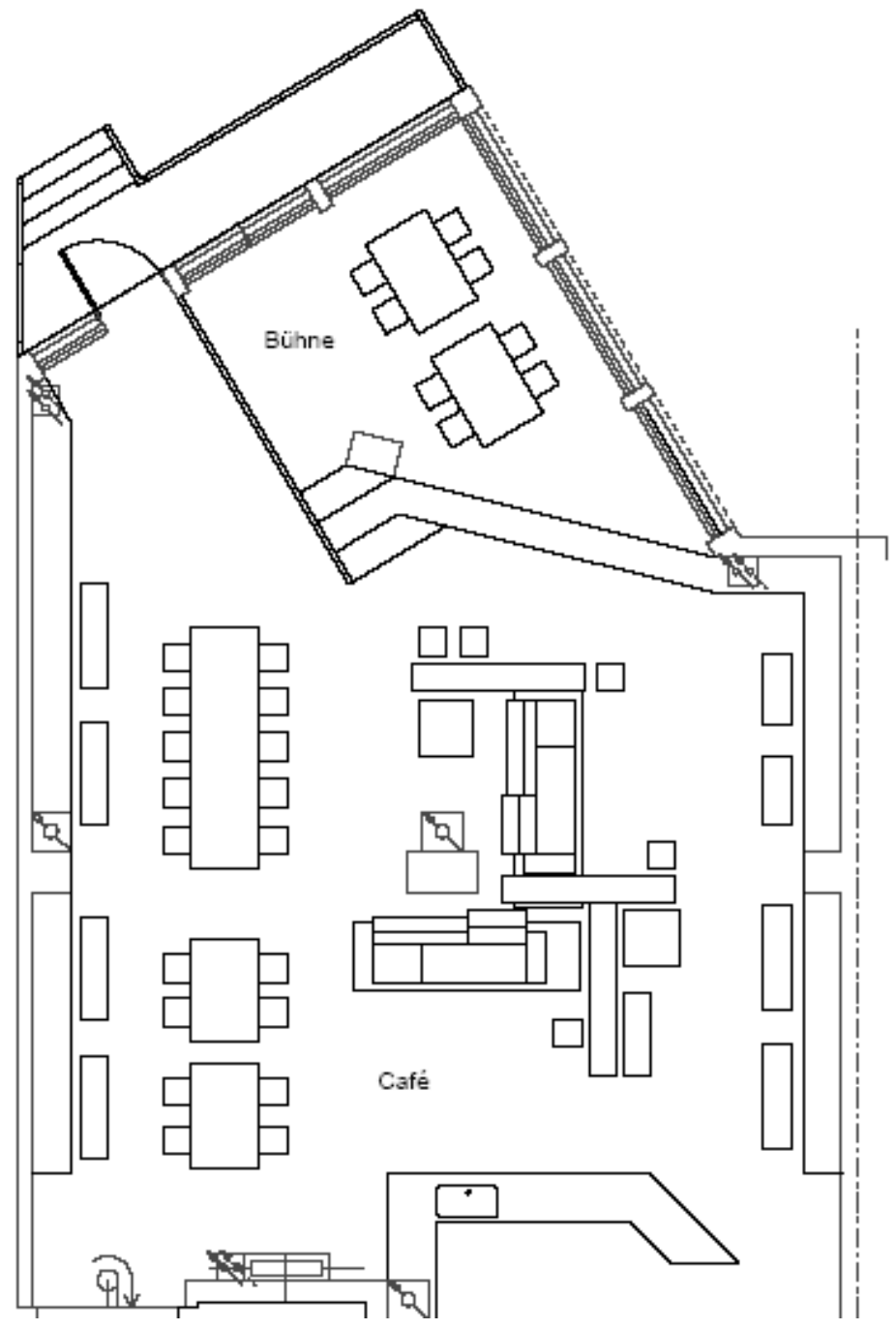

Quelle: Tonia Welter, Betahaus Berlin 
Das Konzept des Co-working ist jedoch so flexibel angelegt, dass es sich an die jeweiligen Gegebenheiten und Veränderungen anzupassen weiß. Auffällig ist, dass sich an diesen Orten schnell ein soziokulturelles „Ökosystem“ entwickelt. Christoph Fahle und Tonia Welter vom Betahaus in Berlin:

In diesem Möglichkeitsraum Betahaus haben wir zusätzlich zu der Gesamtorganisation des Raumes auch die Funktion eines „Sozialstatikers“. Wir müssen die unterschiedlichen Interessen mit unseren Ansprüchen und denen der anderen Nutzer des Raums ausbalancieren.

\section{Neue Arbeit in Wertegemeinschaften}

Das Konzept der Co-working Spaces hat unterschiedliche Ausprägungen. Zum einen gibt es Orte, deren Identitäten durch ihre Betreiber herausgearbeitet und mit einer impliziten Wertestruktur versehen werden. Hier wird der Ort gegebenenfalls auch als Marke aufgebaut, unter deren Dach unterschiedliche dort entstandene, bzw. durchgeführte Projekte präsentiert werden können. Solche Orte sind in der Regel stark Community-gebunden und treten auch nach außen als eine Wertegemeinschaft auf (in Berlin z. B. der selfHUB für Social Entrepreneurs, das Betahaus für kreative Freelancer). Andere Initiativen wiederum sehen sich eher als Bindeglied zwischen Mensch und Ort mit dem Ziel, ,neue Orte für neues Arbeiten“ zu entwickeln. Hier ist der Anspruch weniger ideologisch denn funktional; Sebastian Sooth vom Hallenprojekt in Berlin:

Wir wollen bessere soziale Interaktion unterstützen. Wir glauben, dass das Coworking nicht nur eine bessere Arbeitsform für kreative Freelancer ist, sondern dass es eine bessere Arbeitsform für jeden ist, der mit einem Rechner arbeitet, egal ob Anwalt, Buchhalter oder Ingenieur, egal ob festangestellt oder selbständig. Es müssen nicht zwangsweise nur die Leute aus der Kreativwirtschaft sein.

\section{Schwärme im Kontext von Betakulturen}

Die Kultur dieser neuen Arbeit reagiert auf die Tatsache, dass immer mehr Menschen die digitalen Möglichkeiten nutzen wollen, um selbstbestimmt und mobil zu arbeiten. Sie wollen dies mit dem Anspruch auf Selbstentfaltung und Autonomie tun, ohne dabei gleichzeitig auf den Mehrwert eines sozialen sowie professionellen Umfeldes zu verzichten. Dieser Anspruch der flexiblen Anbindung an ein Kollektiv bei gleichzeitigem Autonomieerhalt entspricht einem Schwarmverhalten. Bei diesem 
Phänomen steht weniger die alte Trennlinie zwischen abhängig arbeitenden Angestellten und Selbständigen im Vordergrund. Vielmehr verläuft eine neue Trennlinie zwischen Leuten, die ständig erreichbar sein müssen, und Leuten, die selber steuern können, wann und mit wem sie kommunizieren. Letztere werden in der Betakultur der Co-working Spaces, die prozessuales, vernetztes ad hoc-Arbeiten möglich macht, einen geeigneten Entfaltungsspielraum finden. Christian Heller vom Hallenprojekt in Berlin:

Wir gehen davon aus, dass sich die einzelnen Bausteine der Arbeit flexibilisieren, dass es also kein festes Paket mehr gibt, in dem alles vordefiniert wäre, also wer mit wem wann und wie zusammenarbeitet, sondern dass sich alles bedarfsorientiert immer wieder neu zusammenorganisieren muss, und dafür wollen wir die Infrastruktur anbieten. 

KOMPETENZEN 



\section{Kompetenz}

\section{HARALD A. MIEG}

Wenn die Kreativwirtschaft von kurzlebigen Innovationen, Trends und Modezyklen bestimmt wird, wie lassen sich dann Kompetenz und Kompetenzentwicklung in der Kreativwirtschaft verstehen? Welche Bedeutung hat Ausbildung in der Kreativwirtschaft? Brauchen wir neue Bildungsträger?

Der folgende Beitrag gibt einen Einblick in das Phänomen der Kompetenz und versucht auf dieser Grundlage die Randbedingungen für Kompetenz in der Kreativwirtschaft zu skizzieren. Auf die Darstellung von Praxisbeispielen wird verzichtet, da dies in den anderen Kapiteln erfolgt. Hinzuweisen ist insbesondere auf das gelungene Beispiel der Popkulturförderung in Mannheim (vgl. den Artikel von Sebastian Dresel in diesem Band). In Mannheim findet Kompetenzentwicklung auf verschiedenen Ebenen statt:

- auf der Ebene der Stadtverwaltung und -politik, der es gelungen ist, Baden-Württembergs Popakademie in Mannheim anzusiedeln und ihr ein in jeder Hinsicht förderliches Umfeld zu schaffen;

- auf der Ebene individueller, professioneller Ausbildung im Bereich Popmusik, die nicht nur die unternehmerische Komponente einbezieht, sondern direkt den Weg in die Unternehmensgründung eröffnet (im Musikpark);

- auf der Ebene der Stadt, welche neben der Industriekultur auch eine Tradition der Musikausbildung aufweist und - für Kreativwirtschaft fast unabdingbar - eine gelebte Multikulturalität besitzt.

Die Diversität der Kompetenzträger vom Individuum bis hin zur Stadt ist nicht untypisch in der Kreativwirtschaft und soll im Folgenden näher beleuchtet werden. Doch zuerst zum Begriff der Kompetenz. 


\section{Begriff der Kompetenz}

Der Begriff der Kompetenz enthält mehrere Bedeutungsrichtungen. Kompetenz meint zum einen eine Fähigkeit, etwa im Beispiel der Sprachkompetenz. Kompetenz kann zum anderen auf eine Zuständigkeit abzielen, etwa im Beispiel der Richtlinienkompetenz des deutschen Bundeskanzlers, der den Rahmen der Regierungspolitik festzulegen hat. Eine weitere Facette von Kompetenz besteht in der Motivation, bestimmte Handlungen auszuüben. So ist ein kompetenter Ansprechpartner nur jemand, der auch bereit ist, Auskunft zu geben. In unserem Zusammenhang liegt das Augenmerk auf Kompetenz im Sinne einer Fähigkeit. Doch ist es von Vorteil, bei der Diskussion um Kompetenz die anderen Bedeutungsrichtungen mitzudenken.

Kompetenz lässt sich abgrenzen von Qualifikation. Qualifikation ist als zertifizierte Kompetenz zu verstehen. Qualifikationen können formal gefasst sein, wie im Fall von Zeugnissen und Diplomen. Qualifikationen können aber auch auf mehr oder weniger unausgesprochenen Übereinkünften bestehen. Die Grundidee ist, dass die qualifizierenden Fähigkeiten bestimmten Anforderungen entsprechen. In der Berufsbildungsforschung (vgl. z. B. Rauner 2005) schwenkte in den letzten Jahrzehnten der Fokus der Betrachtung weg von Qualifikationen hin zu Kompetenzen. Dies hängt auch mit der Entwicklungsdynamik und Unklarheit der Berufsbilder zusammen, wie wir sie in der Kultur- und Kreativwirtschaft antreffen.

\section{Diversität der Kompetenzträger}

Es gibt drei Sorten von Kompetenzträgern (vgl. auch Abbott 1991). Kompetenz kann sich verkörpern in:

- Individuen

- Organisationen

- Produkten

Ein einfaches Beispiel: Kochkompetenz finden wir üblicherweise bei gelernten Köchen. Jedoch verkörpern auch Fast-Food-Restaurantketten Kochkompetenz, wobei kein einziger der Mitarbeiter der Organisation über Kochfertigkeit verfügen muss. Nicht unwichtig ist der Hinweis, dass auch Produkte Kompetenzträger sein können, im Fall des Kochens sind dies: Rezepte, Kochbücher, intelligente Backöfen usw. Ein zentraler Produkt-Kompetenzträger in der heutigen Zeit ist der Computer. Ohne die Digitalisierung ist auch der Aufschwung der Kreativwirtschaft in 
den 1990er Jahren nicht denkbar. Digitale Bild- und Musikbearbeitung ersetzen heute Kompetenzen, die früher an Spezialisten delegiert werden mussten.

Die Frage der Kompetenz von Organisationen sei hier im Folgenden etwas näher betrachtet. Bezüglich der Organisationsformen der Kreativwirtschaft ist meist von Netzwerken die Rede, zum Beispiel mit Bezug auf die Arbeiten von Grabher (z. B. 2004; 2006). Dies ist im Prinzip richtig, im Folgenden sollen jedoch explizit Organisationen und nicht Netzwerken behandelt. Der Organisationsbegriff mag im Vergleich zu dem Netzwerkbegriff allgemeiner und weniger modern klingen, er hat jedoch den Vorteil, dass sich damit klar die Formen von überindividueller Arbeitsteilung ansprechen lassen. Unternehmen sind eine Organisationsform von Arbeitsteilung; sie lassen sich zwar auch als Netzwerke verstehen, damit geraten jedoch wesentliche Punkte der organisierten Arbeitsteilung (Vertragsbasierung, Unternehmenskultur usw.) in den Hintergrund. Tabelle 1 zeigt eine Auswahl von Organisationsformen, die im Weiteren diskutiert werden sollen. Die Liste ist keineswegs vollständig, zum Beispiel fehlen Firmennetzwerke oder die öffentliche Verwaltung. Auch im Bezug auf die Kreativwirtschaft ist die Auswahl nicht umfassend. Es fehlt beispielsweise die Organisation von Freiwilligenarbeit, die auch für die Kreativwirtschaft nicht unwichtig ist. Die hier getroffene Auswahl soll relevante, generelle Unterschiede in der Organisation von Arbeitsteilung deutlich machen.

Gruppen können als Mengen von Individuen, die miteinander in Kontakt stehen, aufgefasst werden. Informationsverarbeitung in nichthierarchischen Gruppen folgt meist dem Mehrheitsprinzip: Es werden nur die Informationen zur Kenntnis genommen und im Gruppengedächtnis behalten, die die meisten betreffen, in der Regel ist dies die gemeinsame Gruppenbiographie. Nichtgeteiltes Sonderwissen einzelner Mitglieder geht in Gruppen oft verloren, selbst wenn eine Gruppe eine Aufgabe übernimmt (Stewart/Stasser 1995). Das Sonderwissen findet erst Eingang, wenn es zu einer Arbeitsteilung und Rollenverteilung kommt und das Sonderwissen als Wissen eines internen Experten formatiert ist.

Unter Arbeitsteilung entwickeln Gruppen ein sogenanntes ,transaktives Gedächtnis“ (Wegner 1987): neue Informationen werden an die zuständigen (,kompetenten“) Mitglieder weitergeleitet und nur dort gespeichert. Das Wissen arbeitsteiliger Gruppen wird somit distribuiert. Auf diese Weise kommt es zu einer Art Kompetenzregulation in Gruppen.

Im Bereich der Kreativwirtschaft finden wir so genannten Communities of Practise (Brown/Duguid 1991; Lave 1991): Lokale Netzwerke 
von Personen bzw. Firmen, welche durch flexible Arbeitsteilung eine Gruppenkompetenz entwickeln, z. B. in der norditalienischen Schuhund Bekleidungsindustrie, die in großen Teilen von Gruppen aus Kleinund Familienbetrieben getragen wird.

Unternehmen haben gegenüber einfachen Gruppen den Vorteil, dass hier die Arbeitsteilung klarer organisiert werden kann. Dies liegt vor allem an der scharf trennenden Formalität der Mitgliedschaft: Per Vertrag gehört man einem Unternehmen an, oder aber mehr. Gruppen sehen sich oftmals mit dem Problem konfrontiert, ihre eigenen Mitglieder zu motivieren. In Unternehmen können die Kompetenzen (in einem weiten Sinne) umfassend geregelt werden: Fähigkeiten und Zuständigkeiten werden zugewiesen, die Arbeitsmotivation wird durch Gehalt und Anstellung geregelt.

Neben der Kompetenzregelung haben Unternehmen den Vorteil, dass sie in hohem Maße Kapital- und Arbeitsressourcen bündeln können, beispielhaft sichtbar in der industriellen Produktion. Unternehmen sind ab einer gewissen Größe notwendigerweise hierarchisch organisiert. Kreativwirtschaft ist vielfach projektbasierte Arbeit (vgl. z. B. DeFillippi/Arthur 1998; DeFillippi 2002, Grabher 2004). Diese Organisationsform ist nicht immer einfach in hierarchisch organisierten Unternehmen umsetzen, zumal dann nicht, wenn Flexibilität und rasche Anpassungen erforderlich sind.

Kommen wir zur dritten Organisationsform, den Städten. Großstädte gelten als ein Nährboden für Kreativwirtschaft. Auch Städte müssen Arbeitsteilung organisieren. Gegenüber Unternehmen und Gruppen sind Städte jedoch stark im Nachteil, weil sie sich ihre Mitglieder - Einwohner - nur bedingt aussuchen können. Ein weiterer Nachteil ist die Immobilität von Städten: Städte können weder räumlich ausweichen, noch können sie sich einfach auflösen, wie das für Gruppen oder Unternehmen möglich ist.

Mit Blick auf Kreativwirtschaft erweist sich das Organisationsproblem von Städten als gewisser Vorteil: Wie Georg Simmel bereits zu Beginn des 20. Jahrhunderts konstatierte, ist die Anonymität eine konstitutives Element von Stadtkultur. In Großstädten kann man dank einer gewissen Anonymität zwischen Netzwerken wählen. Großstädte sind in dieser Hinsicht gewaltige Ressourcenpools mit einer unorganisierten Diversität: Jobmöglichkeiten, Freizeitangebote, Beziehungsformen. Die Ressourcendichte von Großstädten - im Sinne einer ,,institutional thickness“ (Sydow/Staber 2002) - ermöglicht vertikale und horizontale Mobilität, das heißt: Großstädte bieten das Umfeld für Karrieren wie auch für das rasche Wechseln der Wohnadresse. Anders als Unternehmen konkurrieren Städte nicht am Markt miteinander, eine gewisse Leis- 
tungskontrolle erfolgt über das Ansehen hinsichtlich Einfluss, Wohlstand oder Lebensqualität. Seit der Jahrtausendwende bestimmt die Idee der Creative City (Landry 1996) den Ansehenswettbewerb unter Städten. Mannheim hat es geschafft, Anerkennung als „Kompetenzzentrum“ für Popmusik zu finden. Dass auch die Leitidee der Creative City viel Raum für Interpretation lässt, zeigt der Umstand, dass manche Städte darunter eine Form von Wirtschaftspolitik verstehen, während andere Städte eine Profilierung als Creative City im Kulturbereich anstreben.

Tabelle 1: Organisationsformen

\begin{tabular}{|c|c|c|c|c|c|}
\hline & Gruppen & $\begin{array}{l}\text { Unterneh- } \\
\text { men }\end{array}$ & Städte & $\begin{array}{l}\text { Wertschöp- } \\
\text { fungsketten }\end{array}$ & Professionen \\
\hline $\begin{array}{l}\text { Mitglied- } \\
\text { schaft }\end{array}$ & persönlich & $\begin{array}{l}\text { formal, } \\
\text { distinkt }\end{array}$ & $\begin{array}{l}\text { Einwohner } \\
\text { (anonym) }\end{array}$ & funktional & $\begin{array}{l}\text { fachlich, } \\
\text { kollegial }\end{array}$ \\
\hline $\begin{array}{l}\text { Komptenz- } \\
\text { organisation }\end{array}$ & $\begin{array}{l}\text { Regelungs- } \\
\text { bedarf }\end{array}$ & $\begin{array}{l}\text { geregelt } \\
\text { (hierar- } \\
\text { chisch) }\end{array}$ & sozial & $\begin{array}{l}\text { klar } \\
\text { (funktional) }\end{array}$ & $\begin{array}{l}\text { Sach- } \\
\text { motivation }\end{array}$ \\
\hline $\begin{array}{l}\text { Geteiltes } \\
\text { Wissen }\end{array}$ & $\begin{array}{l}\text { Gruppen- } \\
\text { biographie }\end{array}$ & $\begin{array}{l}\text { Unterneh- } \\
\text { menskultur }\end{array}$ & $\begin{array}{l}\text { Nachbar- } \\
\text { schaft }\end{array}$ & Prozess & $\begin{array}{l}\text { Fachgebiet, } \\
\text { abstrakt }\end{array}$ \\
\hline $\begin{array}{l}\text { Ungeteiltes } \\
\text { Wissen }\end{array}$ & (distribuiert) & Distribuiert & anonym & $\begin{array}{l}\text { teilfunk- } \\
\text { tions- } \\
\text { spezifisch }\end{array}$ & $\begin{array}{l}\text { Spezialge- } \\
\text { biet }\end{array}$ \\
\hline $\begin{array}{l}\text { Räumliche } \\
\text { Dimension }\end{array}$ & Kontaktnetz & $\begin{array}{l}\text { Schwer- } \\
\text { punkte }\end{array}$ & $\begin{array}{l}\text { immobil, } \\
\text { konzentrisch }\end{array}$ & flexibel & virtuell \\
\hline $\begin{array}{l}\text { Zeitliche } \\
\text { Dimension }\end{array}$ & beliebig & $\begin{array}{l}\text { kurz- bis } \\
\text { mittelfristig }\end{array}$ & langfristig & $\begin{array}{l}\text { kürzer als } \\
\text { die Unter- } \\
\text { nehmen }\end{array}$ & langfristig \\
\hline $\begin{array}{l}\text { Ressourcen- } \\
\text { pool }\end{array}$ & kontingent & Geplant & divers & $\begin{array}{l}\text { instrumen- } \\
\text { tell, ökono- } \\
\text { misiert }\end{array}$ & $\begin{array}{l}\text { bereichs- } \\
\text { spezifisch }\end{array}$ \\
\hline $\begin{array}{l}\text { Leistungs- } \\
\text { kontrolle }\end{array}$ & $\begin{array}{l}\text { individua- } \\
\text { lisiert }\end{array}$ & $\begin{array}{l}\text { Markt } \\
\text { (Überleben) }\end{array}$ & $\begin{array}{l}\begin{array}{l}\text { Ansehen } \\
\text { (der Stadt) }\end{array} \\
\end{array}$ & $\begin{array}{l}\text { Profitabili- } \\
\text { tät, Effizienz }\end{array}$ & Peer Review \\
\hline $\begin{array}{l}\text { Themen der } \\
\text { Kreativ- } \\
\text { wirtschaft }\end{array}$ & $\begin{array}{l}\text { Commu- } \\
\text { nities of } \\
\text { practice }\end{array}$ & $\begin{array}{l}\text { projektba- } \\
\text { sierte Arbeit }\end{array}$ & $\begin{array}{l}\text { Creative } \\
\text { Cities }\end{array}$ & $\begin{array}{l}\text { Creative } \\
\text { fields }\end{array}$ & $\begin{array}{l}\text { lokale } \\
\text { „Märkte“" }\end{array}$ \\
\hline
\end{tabular}

Quelle: Eigene Darstellung

Wertschöpfungsketten sind eine Organisationsform von entwickelter, arbeitsteiliger Wirtschaftstätigkeit. Porter (1990) hat gezeigt, wie wichtig Wertschöpfungsketten für die Bildung von industriellen Clustern sind. In der Kulturindustrie - etwa Musik und Film - haben sich solche hochdifferenzierten Wertschöpfungsketten herausgebildet. Beim Film gibt es meist mehrere Ketten aus Produktion, Vermarktung und Kinobetrieb, an denen eine Vielzahl von Unternehmen beteiligt sind. Wertschöpfungsketten sind nicht einfach Netzwerke sondern Kooperationen auf Zeit, die einer klaren Leistungsbewertung nach Profitabilität und Effizienz unterliegen. 
Für Unternehmen geht es zwar auch um Profit, letztlich zählt aber das Überleben, beispielsweise unter Nutzung von Subventionen. In Gruppen und Netzwerke generell ist der Nutzen - und damit das Leistungskriterium - stark individualisiert, d. h. in Gruppen können sehr unterschiedliche, oft unklare Motivationen zusammen kommen.

Wertschöpfungsketten sind hingegen klare Zweckgemeinschaften. Was bei Porter die Cluster sind, sind bei Scott (2006) - mit Bezug auf die Kreativwirtschaft - kreativen Felder (creative fields): Kreative Felder sind Orte oder Gebiete, an denen sich Firmen, Unternehmertum und Innovationstätigkeit konzentrieren und an denen - stets in Nähe zu industrieller Produktion - eine ständige Umdeutung kultureller, symbolischer Produkte stattfindet. Viele der erfolgreichen Wissenschafts- und Technologieparks wie das Research Triangle stellen kreative Felder dar, ebenso z. B. Medien-Standorte wie die Filmproduktion in Mumbai.

Als ein Sonderfall sei hier noch die Organisationsform der Profession vorgestellt. Professionen sind organisierte Berufsgruppen (vgl. Freidson 2001; Mieg 2005). Mitglieder einer Profession teilen ein berufsspezifisches Fachwissen. Von daher sind Berufsgruppen nicht lokal gebunden, sie bilden oft internationale Netzwerke, die von Tagungen oder Ausbildungsveranstaltungen getragen werden. Der Organisationsgrad von Professionen und ihr gesellschaftlicher Einfluss können sehr unterschiedlich sein. Mediziner sind sehr gut organisiert und wissen über Berufsverbände auch auf die Politik Einfluss zu nehmen. Weniger gut sichtbar sind z. B. die Organisationen von Umweltfachleuten oder Experten statistischer Ämter.

Viele der Funktionen, welche Professionen übernehmen können, etwa die Ausbildung oder die Kontrolle des Zugangs zu bestimmten Tätigkeitsfeldern, werden auch von anderen gesellschaftlichen Instanzen geregelt. Das wesentliche Leistungskriterium für Professionen liegt heute im Monopol der Bewertung. Professionen haben ein gewisses Definitionsmonopol für Standards bzw. best practice in einem Tätigkeitsfeld. Diese Bewertungsfunktion erfolgt über das so genannte Peer review: die Leistung eines Professionsmitglied wird durch andere Professionsmitglieder bewertet.

Im Fall der Creative Industries können lokale Netzwerke bzw. lokale Märkte eine Bewertungsfunktion übernehmen. Dies erfolgt weniger über erzielte Preise als über eine im lokalen Netzwerk kommunizierte und stabilisierte Leistungsbewertung (Lange/Mieg 2008). 


\section{Modelle individueller Kompetenzentwicklung}

In der Forschung gibt es verschiedene Modelle zur Beschreibung und Erklärung individueller Kompetenzentwicklung (Rauner 2005). In Tabelle 2 werden drei Modellvarianten vorgestellt (vgl. Woschnack/Mieg 2003). Das Modell „,formale Qualifikation“ entspricht dem Standardmodell von Schule. Kompetenzentwicklung bedeutet hier, dass durch Ausbildung Wissen vermittelt wird. Die Entwicklungslogik ist hierbei additiv und modular: Fehlendes Wissen wird durch zusätzliche Ausbildung entsprechender Wissensblöcke vermittelt. Kompetenzentwicklung im Modell „Expertise“ beruht auf langjähriger bereichsspezifischer Erfahrung, zumeist in Form von „Berufserfahrung“. Expertise ist Gegenstand der Psychology of Expertise (vgl. Ericsson/Charness/Feltovich/Hoffman 2006) und bezieht sich auf individuelle Könnerschaft. Diese Könnerschaft (oder auch Expertentum) zeigt sich daran, dass jemand Aufgaben und Probleme eines bestimmten Bereichs meistern kann. Die Forschung hat mittlerweile zahlreiche generelle Befunde erbracht. Hierzu gehört zum Beispiel die 10-Jahres-Regel, wonach es etwa zehn Jahre zielgerichteten Übens und Tätigseins (,deliberate practice“) bedarf, um entsprechende Expertise zu entwickeln. Ein weiterer Befund ist die strikte Bereichsspezifizität: Expertise in einem Bereich lässt sich so gut wie gar nicht auf einen anderen Bereich übertragen. Von daher ist die Entwicklungslogik die des Spezialistentums.

Tabelle 2: Modelle der Kompetenzentwicklung

\begin{tabular}{|l|l|l|l|}
\hline & $\begin{array}{l}\text { formale Qualifika- } \\
\text { tion (Wissen) }\end{array}$ & $\begin{array}{l}\text { Expertise } \\
\text { (Erfahrung) }\end{array}$ & $\begin{array}{l}\text { Schlüsselqualifikation } \\
\text { (Person) }\end{array}$ \\
\hline Leistungskriterium & Wissen & $\begin{array}{l}\text { „Meistern“, } \\
\text { Problemlösen }\end{array}$ & $\begin{array}{l}\text { Lerngeschwindigkeit, } \\
\text { Kommunikations- } \\
\text { kompetenz }\end{array}$ \\
\hline Kompetenzquelle & Ausbildung & $\begin{array}{l}\text { Erfahrung } \\
\text { (10-Jahres-Regel; } \\
\text { Bereichs- } \\
\text { spezifizität) }\end{array}$ & $\begin{array}{l}\text { Person (Intelligenz) + } \\
\text { „Schliff“ }\end{array}$ \\
\hline Entwicklungslogik & additiv & $\begin{array}{l}\text { spezialisiert, } \\
\text { vertiefend }\end{array}$ & $\begin{array}{l}\text { Persönlichkeits- } \\
\text { entwicklung }\end{array}$ \\
\hline Nachweis & Zeugnisse, Noten & Berufserfahrung & Biographie \\
\hline $\begin{array}{l}\text { relevante Themen } \\
\text { der Kreativwirt- } \\
\text { schaft }\end{array}$ & $\begin{array}{l}\text { hohe Innovativität } \\
\text { (Kurzlebigkeit des } \\
\text { Wissens) }\end{array}$ & $\begin{array}{l}\text { Kurzlebigkeit und } \\
\text { Entgrenzung der } \\
\text { Bereiche/Märkte }\end{array}$ & $\begin{array}{l}\text { Risikokarrieren, Unter- } \\
\text { nehmertum, Selbstorga- } \\
\text { nisation }\end{array}$ \\
\hline
\end{tabular}

Quelle: Eigene Darstellung

Kompetenzentwicklung nach dem Modell „Schlüsselqualifikation“ beruht auf der Grundfähigkeit, lernen zu lernen (Mertens 1974). In eher weiter Auslegung handelt es sich bei Schlüsselqualifikation um eine vermittelbare Fähigkeit, in engerer Auslegung beruht diese auf einer 
Generalfähigkeit gewisser Personen, die sich in Lerngeschwindigkeit und Kommunikationskompetenz ausdrückt. Die Entwicklungslogik entspricht der Maxime der Trainee-Programme führender Unternehmensberatungen: Hauptsache man hat Leute, die sich bisher in irgendeinem Bereich als exzellent erwiesen haben; das branchenspezifische Wissen wird on the job vermittelt.

Ein zentrales Thema in der Kreativwirtschaft sind die Risikokarrieren. Viele Protagonisten der Kreativwirtschaft sind oder werden zu SoloUnternehmern, die sich und ihre symbolischen Produkte oder Dienstleistungen vermarkten müssen. Unternehmerische Fähigkeit zur Selbstorganisation wird hierbei zu einer Art Schlüsselqualifikation (vgl. z. B. Rae 2002, 2004). Lange (2007) spricht von Culturepreneurs. Das mit der Kreativwirtschaft verbundene Risiko beruht auf der Kurzlebigkeit und Entgrenzung vieler Teilbereiche (z. B. Games). Für die klassischen Bereiche der bildenden und darstellenden Kunst, von Musik über Malerei zu Theater, gilt nachweislich die 10-Jahres-Regel der Kompetenzentwicklung, d. h. des Meisterwerdens (vgl. Ericsson et al. 2006). In allen Bereichen, die durch IT geprägt oder getrieben werden, sind die Lebenszyklen der definierenden Bereichsgrundlagen inzwischen deutlich unter 10 Jahre verkürzt. Erfahrungsgestützte Kompetenzentwicklung im Sinne von Expertise ist in diesen Bereichen kaum möglich.

Noch ein Wort zu formalen Qualifikationen in der Kreativwirtschaft: Mit der rasanten Entwicklung der Tätigkeitsfelder kann das öffentliche System der Berufsbildung nicht mithalten. Es wurden zwar eine Reihe von neuen Ausbildungsgängen ins Leben gerufen, z. B. im IT-Bereich, auch hat die Einführung des Bachelor-/Master-Systems an den Hochschulen zu einer gewissen Flexibilisierung geführt: Doch ist in diese öffentlichen Systeme akademischer Berufsausbildung eine notwendige Verzögerung und Trägheit eingebaut, nicht zuletzt weil öffentliche Mittel damit verbunden sind. Was wir hingegen in Teilbereichen der Kreativwirtschaft beobachten ist eine gewisse Re-Professionalisierung: Ausbildung wird von selbstorganisierten Einrichtungen der Kreativen definiert and angeboten. Es entstehen außeruniversitäre, mehr oder minder kommerzialisierte „Akademien“ und mit der Zeit neue Standards.

\section{Exkurs: Neue Organisationsformen?}

Im Zusammenhang mit Kreativwirtschaft wird oft behauptet, dass neue, flexible, temporäre Organisationsformen entstünden (z. B. bei Djelic/ Ainamo 1999, oder in Gernot Grabhers Arbeiten). Unbestreitbar ist die neue Bedeutung, welche die Organisationsform des Netzwerkes im Zu- 
sammenhang mit dem Internet gewonnen hat. Hier bedienen sich nicht nur bestehende Netwerke, z. B. wissenschaftliche oder professionelle Vereinigungen, eines neues Mediums, sondern es entstehen neue internetgestützte Gruppierungen. Das Internet hat zudem entscheidend den Zugang zu Wissen und Netzwerken erleichtert und ist damit ein unabdingbares Werkzeug für Kreativunternehmer, culturepreneurs. Der Bedeutungsgewinn der Netzwerke rechtfertigt jedoch nicht, auch Unternehmen oder andere Organisationsformen wie z. B. Städte als (bloße) Netzwerke aufzufassen.

Ein vielfach zitiertes Phänomen im Zusammenhang mit der Kreativwirtschaft sind die neue „Agenten“, d. h. Zwischenhändler und Vermittler, als „Creativity Broker“ (Bilton/Leary 2002) oder „Boundary spanners“ (Gander/Haberberg/Rieple 2007), welche mitunter sogar eine gewisse Kontrollfunktion im Informationsfluss übernehmen. Die Agenten verfügen über Schnittstellenkompetenz, Kompetenz diesmal in vollem Umfang des Begriffes verstanden: sie sind fähig, zuständig und willens, eine Vermittlungsfunktion zu übernehmen.

So war es ein Erfolgselement des Mannheimer Modells der Popkulturförderung, die Stelle eines Beauftragten der Stadt für Musik und Popkultur zu schaffen und ausdrücklich mit Netzwerk- und Vermittlungsarbeit zu beauftragen. Im Kunst- und Kulturgewerbe sind Agenten bzw. Manager von jeher eine bekannte Größe. Die Beschleunigung der gesellschaftlichen Prozesse und deren wachsende Komplexität und Vielfalt machen Einsatz von Vermittlern und Zwischenhändlern heute fast unausweichlich.

Was wir zudem in der Kreativwirtschaft beobachten können, sind neue Ansätze zur Professionalisierung, und zwar sowohl der Individuen als auch einiger Teilfelder (z. B. Games). Dies bedeutet zweierlei: Erstens den Übergang zu bezahlter Arbeit und eine notwendige Ökonomisierung. Zweitens eine soziale Schließung: Es entstehen Gruppierung mit Bewertungsmonopolen und spezifisches Expertentum. Ob diese Professionalisierung zu neuen Professionen führt, bleibt abzuwarten. Das Internet unterstützt zwei gegenläufig wirkende Trends: Einerseits die Demokratisierung der Leistungsbewertung, etwa durch Online-Kundenbewertungen, und andererseits die Bildung exkludierender professionalisierter Expertennetzwerke. Das Entstehen neuer Kreativ-Professionen hängt letztlich davon ab, ob sich für bestimmte Teilbereiche der Kreativwirtschaft ein Korpus an abstraktem, formalisierbarem Wissen aufbauen lässt. Denn nur in Bezug auf ein solches Wissenssystem lassen sich Bewertungsmonopole definieren. 


\section{Resümee}

Die Betrachtung von Kompetenz und Kompetenzentwicklung im Kontext von Kreativwirtschaft wirft einige grundlegende Fragen auf, auf die wir abschließend kurz eingehen wollen.

Frage: Wie lässt sich die Kompetenzentwicklung konzeptionell beschreiben, wenn die Kreativwirtschaft von kurzlebigen Innovationen, Trends, Modezyklen usw. bestimmt wird?

Antwort: Derzeit ist in der Kreativwirtschaft die Herausbildung von Kompetenz in einem starken Sinne von Expertise sehr erschwert. Auch so genannte Skills, d. h. professionell-,,handwerkliche“, technische Fähigkeiten, z. B. für Webdesign, haben nur begrenzten Sonderwert, da zunehmend Spezialkompetenzen auch käuflich erworben werden können, vor allem in Form von Software. Große Bedeutung haben in der Kreativwirtschaft derzeit Schlüsselqualifikationen im Sinne von intelligenten Generalfertigkeiten gewonnen. Diese sind nötig, um Risikokarrieren zu steuern und als Kreativunternehmer erfolgreich zu sein. Die Fähigkeit zur unternehmerischen Selbstorganisation spielt hierbei eine große Rolle.

Frage: Inwiefern sind in der Kreativwirtschaft Kompetenzen institutionell stabil gebunden?

Antwort: In der Kreativwirtschaft ist derzeit eine stabile institutionelle Bindung von Kompetenzen nur den bereits länger bestehenden Ausbildungsinstitutionen, Netzwerken und Unternehmen gegeben. Dies deckt vermutlich nur ein Segment des Spektrums von Kompetenzen in diesem Feld ab. In der Kreativwirtschaft dominiert die projektbasierte, netzwerkgestützte Arbeit. Das Wissen und die entsprechenden Kompetenzen sind individualisiert bzw. instabil in den Netzwerken gebunden. Dies ist auch ein Grund dafür, dass sich eine ortsabhängige Ressourcendichte förderlich für die Kreativwirtschaft auswirkt. Von daher kann man sagen, dass gewisse Städte oder „kreative Felder“ (Scott 2006) relevante Kompetenzen stabil binden.

Frage: Wie müssten etablierte Wissenseinrichtungen, etwa Hochschulen verschiedenster Ausrichtung - auf entgrenzte Wissensnetze reagieren?

Für die Ausbildung in Richtung Kreativwirtschaft ergeben sich zahlreiche Anschlussmöglichkeiten in den bestehenden Studiengängen. Diese bestehen zum einen in der Förderung von Schlüsselqualifikationen und unternehmerischer Selbstorganisation, beispielsweise mit einem Kunst- oder Informatikstudium. Zum anderen in der Einrichtung spezialisierter Teil- und Nebenfächer, die bestimmte Teilfragen der Kreativwirtschaft vertiefen, etwa Urheberrecht oder Kunstauktionsmarkt. Offen 
soll an dieser Stelle die Frage bleiben, ob Kreativität selbst als eine förderungsfähige Kompetenz aufgefasst werden kann - die hängt sehr stark vom Begriff der Kreativität ab. Eine andere Frage betrifft die Ausbildung hinsichtlich für das Schnittstellenmanagement in der Kreativwirtschaft. Hier gibt es bereits entsprechende Master-Studienangebote zum Kreativ- oder Kunstmanager. Den Erfolg solcher Ausbildungen wird die Praxis der Kreativwirtschaft weisen müssen.

Das Beispiel des Mannheimer Modells der Popkulturförderung (vgl. Artikel von Sebastian Dresel in diesem Band) zeigt, wie erfolgreiche Kompetenzentwicklung im Bereich Kreativwirtschaft aussehen kann:

- Ausbildung von Skills, d. h. professionell-handwerklichen Fähigkeiten, verknüpft mit der Einübung unternehmerischer Kompetenz als einer Schlüsselqualifikation.

- Verbindung von akademischer Ausbildung (Popakademie) mit einem „Inkubator“, d. h. einer Einrichtung, die jungen Unternehmen sich zu gründen und zu entwickeln hilft (Musikpark).

- Einbindung von Ausbildung und Gründungsförderung in eine Stadtteilentwicklung, die der kreativen Szene bzw. den kreativen Professionals ein anregendes städtisches Umfeld schafft.

Insgesamt gesehen scheint eine wichtige Leitlinie in der Kreativwirtschaft die Professionalisierung von Ausbildung. Dies umfasst nicht nur eine gewisse Ökonomisierung sondern auch die gezielte Förderung professioneller Netzwerke. In Mannheim erhält man nicht einfach eine qualifizierte Ausbildung zum Popmusiker; vielmehr werden PopmusikProfessionals samt ihrer professionellen und unternehmerischen Netzwerke geschaffen.

\section{Literatur}

Abbott, Andrew (1991): „The Future of Professions“. In: Research in the Sociology of Organizations 8, S. 17-42.

Bilton, Chris/Leary, Ruth (2002): „What Can Managers do for Creativity? Brokering Creativity in the Creative Industries“. In: International Journal of Cultural Policy 8, S. 49-64.

Brown, John S./Duguid, Paul (1991): „Organizational Learning and Communities of Practice: Towards a Unified View of Working, Learning and Innovation“. In: Organization Science 2/1, S. 40-57.

DeFillippi, Robert (2002): „Information Technology and Organizational Models for Project Collaboration in the New Economy“. In: Human Resource Planning 25, 4, S. 7-18. 
DeFillippi, Robert/Arthur, Michael (1998): Paradox in project-based enterprise: The case of film making. In: California Management Review 40, S. 1-15.

Djelic, Marie-Laure/Ainamo, Antti (1999): The Coevolution of New Organization Forms in the Fashion Industry: A Historical and Comparative Study of France, Italy and the United States“. In: Organization Science 10, S. 622-637.

Ericsson, Anders K./Charness, Neil/Feltovich, Paul/Hoffman, Robert R. (Hg.) (2006): The Cambridge Handbook of Expertise and Expert Performance, Cambridge/New York: Cambridge University Press.

Freidson, Eliot (2001): Professionalism: The Third Logic, Cambridge: Polity Press.

Gander, Jonathan/Haberberg, Adrian/Rieple, Alison (2007): „A Paradox of Alliance Management: Resource Contamination in the Recorded Music Industry“. In: Journal of Organizational Behavior 28, S. 607624.

Grabher, Gernot (2004): „Learning in Projects, Remembering in Networks?: Communality, Sociality, and Connectivity in Project Ecologies“. In: European Urban and Regional Studies 11 (2), S. 103 123.

Grabher, Gernot (2006): „Trading Routes, Bypasses, and Risky Intersections: Mapping the Travels of ,Networks' between Economic Sociology and Economic Geography“. In: Progress in Human Geography 30 (2), S. 1-27.

Landry, Charles (Hg.) (1996): The Creative City in Britain and Germany, London: Anglo-German Foundation.

Lange, Bastian (2007): Die Räume der Kreativszenen: Culturepreneurs und ihre Orte in Berlin, Bielefeld: transcript Verlag.

Lange, Bastian/Mieg, Harald A. (2008): „Professionalisierungswege und Konstituierungen von ,Märkten " in den Creative Industries“. Geographische Zeitschrift 94 (4), S. 225-242.

Lave, Jean (1991): „Situating Learning in Communities of Practice“. In: Levine, John M./Resnick, Lauren B./Teasley, Stephanie D. (Hg.), Perspectives on Socially Shared Cognition. Washington, DC: American Psychological Association, S. 63-82.

Mertens, Dieter (1974): „Schlüsselqualifikationen. Thesen zur Schulung für eine moderne Gesellschaft“. Mitteilungen aus der Arbeitsmarktund Berufsforschung 7, S. 36-43.

Mieg, Harald A. (2005): „Professionalisierung“. In: Rauner, Felix (Hg.), Handbuch der Berufsbildungsforschung. Bielefeld: Bertelsmann, S. 342-349. 
Porter, Michael E. (1990): The Competitive Advantage of Nations, New York: The Free Press.

Rae, David (2002): „Entrepreneurial Emergence: A Narrative Study of Entrepreneurial Learning in Independently Owned Media Businesses“. In: The International Journal of Entrepreneurship and Innovation 3 (1), S. 53-60.

Rae, David (2004): „Entrepreneurial Learning: A Practical Model from the Creative Industries“. Education + Training 46 (8/9), S. 492-500.

Rauner, Felix (2005): Handbuch der Berufsbildungsforschung, Bielefeld: Bertelsmann.

Scott, Allen J. (2006): „Entrepreneurship, Innovation and Industrial Development: Geography and the Creative Field Revisited“. Small Business Economics 26 (1), S. 1-24.

Stewart, Dennis D./Stasser, Garold (1995): „Expert role assignment and information sampling during collective recall and decision making“. Journal of Personality and Social Psychology 69 (4), S. 619-628.

Sydow, Jörg/Staber, Udo (2002): The Institutional Embeddedness of Project Networks: The Case of Content Production in German Television“. In: Regional Studies 36, S. 215-227.

Wegner, Daniel (1987): „Transactive Memory: A Contemporary Analysis of the Group Mind“. In: Mullen, Brain/Goethals, George R. (Hg.), Theories of Group Behavior. New York: Springer, S. 185-208

Woschnack, Ute/Mieg, Harald A. (2003): „Fachwissen - Expertise Schlüsselqualifikationen: Drei Dimensionen eines integrativen Modells der Qualifikation für professionalisierte Dienstleistungen“. In: Arbeit 12, S. 54-67. 



\section{Popkulturförderung in Mannheim}

\section{SEBASTIAN DRESEL}

Die schlichte Begrifflichkeit ist es, mit deren Hilfe die Systematik des als „Mannheimer Modell“ bezeichneten und hier zu schildernden Förder- und Entwicklungskonzeptes verdeutlicht werden soll. Popkultur, dieser gleichermaßen offene wie spezifische Begriff eröffnet der Stadt Handlungsoptionen und -felder, die auch jenseits einer rein musikalisch verorteten Genre-Auffassung von Popmusik ansetzen und die der Stadt Mannheim einen bis noch vor wenigen Jahren unvorstellbar rasanten, anhaltenden und in dieser Deutlichkeit sicherlich unerwarteten Imageund Strukturwandel ermöglicht haben. Die Offenheit des Themas (samt der geschaffenen und noch zu erörternden Strukturen) erweist sich nicht zuletzt in der selbstredend auch in Mannheim anhaltenden Debatte um den zukünftigen und gegenwärtigen Stellenwert der Kreativwirtschaft als ausgesprochen vorteilhaft.

Auch der zweite Wortbestandteil der Popkulturförderung ist von immenser Bedeutung in diesem Zusammenhang. Stellt sich doch die Frage, wie sich kommunale „Förderung“ von „Pop“ aus aktiv kulturellem und kooperativem Handeln einerseits und vorbereitender bzw. hintergründiger Bearbeitung von Grundlagen zusammensetzt. Grundlagen, auf denen (Pop-)Kultur (auch und in erster Linie von den diversen Popkulturakteuren selbst) entwickelt werden soll und kann. Die Anschlussfrage, ob eine einzelne Kommune oder Region im Rahmen einer zweifelsohne globalen Kultur überhaupt über wirkungsvolle Werkzeuge (im einen wie dem anderen Gesichtspunkt) verfügt und wenn ja, über welche, beantwortet Mannheim eindeutig mit den hier noch zu schildernden Konzepten und ihrer Umsetzung. Oder kurz mit: ja - einige!

Gerade in der sich anhaltend digital vernetzenden Welt (eine bei aller Wiederholung und Floskelhaftigkeit anscheinend ausgerechnet in der 
Kulturpolitik immer noch nicht als offensichtliche Zwangsläufigkeit begriffene Entwicklung) haben sich jenseits der großen Metropolen Entwicklungspotenziale aufgetan, die noch vor kurzem in dieser Form nicht denkbar waren. Die Popstandortfrage nämlich kann sowohl was Produktion aber auch Rezeption angeht dieser Tage längst nicht mehr so eindeutig pro Weltstadt beantwortet werden, wie das auch noch in den 1990er Jahren vollkommen selbstverständlich war.

Popkulturelles „Niveau“ (mittels Vergleich, Überblick, Wettbewerb, ja sogar „Street Knowledge“) oder „Zeitgeist“" kann beinahe überall nachvollzogen werden (was im Vergleich etwa zur Kunst oder zum Theater natürlich hauptsächlich in der Verfügbarkeit und Transportfähigkeit der zentralen Medien Musik und Mode begründet liegt). Wenn man also vor nicht allzu langer Zeit noch davon abhing, in der Nähe des schicksten Plattenladens, Clubs, Konzerthauses, Boutique zu wohnen, um Trends nicht zu verpassen, geht es heute darum, wie man die jederzeit verfügbaren Informationen im ganz großen Zusammenhang globaler Popkultur im eigenen noch so kleinen Dorf nutzt oder besser: Was für Schlüsse man aus ihrer Verfügbarkeit zieht. Dem zunächst gerechtfertigten Einwand, dass die klassischen Printmedien in dieser Rechnung nicht auftauchen, müssen heute die Partizipations- und damit Aktionsmöglichkeiten des interaktiven Netzes entgegengehalten werden.

Auch hieraus leitet sich das für manche Beobachter auf den ersten Blick verwunderliche Mannheimer Selbstbewusstsein ab: Es basiert gerade in der hiesigen Größenordnung auf den vergleichsweise kompakten und kurzen Informations- und Arbeitswegen, die einen Vorteil gegenüber zwar quantitativ vielseitigeren aber eben auch deutlich schwieriger zu koordinierenden Städten oder deren Netzwerken haben.

Um die besagten Arbeitszusammenhänge zu verdeutlichen, bedarf es einer groben organisatorischen Schilderung der Struktur, durch die sich das „Mannheimer Modell“ zunächst charakterisieren lässt. An erster Stelle steht (wenngleich auch nicht in der Abfolge der Ansiedlung) ohne Zweifel die durch das Land Baden-Württemberg, die Stadt Mannheim und einer ganzen Reihe weiterer Gesellschafter getragene Popakademie Baden-Württemberg, deren Arbeitsfeld über das Kernangebot zweier Studiengänge (Popmusikdesign $=$ Musikstudium und Musikbusiness = Wirtschaftsstudiengang) weit hinaus reicht. Eingebettet sowohl in die Popförderung des Landes und darüber hinaus auch in europäische Ausund Weiterbildungsnetzwerke ist die Popakademie längst als europaweites Best-Practice-Beispiel etabliert.

Zum Beginn des Jahres 2009 befindet sich die Akademie in ihrer nächsten Ausbaustufe und eröffnet mit dem SmixLab einen Studiengang (samt digitalem Labor), der sich aufs digitale Musikgeschäft konzen- 
triert. Die Ansiedlung der Akademie hat für die Stadt Mannheim schon auf ganz praktischer Ebene einen ungemein wichtigen Effekt. Der quantitativ überschaubare aber durch die Auswahlverfahren der Akademie konzentrierte jährliche Zuzug kreativer Köpfe hat innerhalb weniger Jahre zu einer greifbaren Veränderung des dargebotenenen und nachgefragten popkulturellen Niveaus geführt - und damit einhergehend eben auch zu einer gesteigerten Strahlkraft des Geschehens. Das ist allein durch den Umstand zu erklären, dass durch den stetigen Zuzug von $\mathrm{Mu}$ sikern und angehenden Musikmanagern der „Druck“ oder besser die Erwartungshaltung auf bzw. an die Szene und deren Angebote massiv zugenommen hat. Aber auch die Tatsache, dass im räumlich begrenzten Stadtteil-Zusammenhang, auf den noch einzugehen sein wird, das gesteigerte „popkulturelle Grundrauschen“ durch die Studentenschaft schon im Straßenbild nachweisbar ist, macht die Veränderung auf den ersten Blick deutlich. Zumindest für den Beobachter mit lokalen Referenzempfindungen.

Die zweite Säule des Modells ist der in der Nachbarschaft der Popakademie angesiedelte und ebenfalls neu (bzw. zunächst) errichtete $\mathrm{Mu}$ sikpark Mannheim, bei dem es sich um das 2003 eröffnete erste Existenzgründerzentrum in der Musikwirtschaft der Bundesrepublik handelt. Die Organisation des Musikparks bemüht sich um möglichst effektive Nutzung der internen Synergieoptionen und bündelt das Angebot der angesiedelten Firmen auch als Agentur nach außen, indem sie das Portfolio der Mieterstruktur zur Kundenakquise nutzt. Gezielte, auf Anforderungen von Kleinstgründungen zugeschnittene Einstiegsangebote ermöglichen potenziellen Gründern (nicht nur aber auch aus der Popakademie) den direkten Einstieg in eine von professionellen Strukturen geprägte aber dennoch kleinteilige Arbeitsumgebung.

Die dritte (und nicht als Gebäude daherkommende) Säule des Mannheimer Modells und das Verbindungsglied zur nicht institutionalisierten vierten (nämlich der „Musikszene“ als solches) ist mit dem Beauftragten für Musik und Popkultur der Stadt Mannheim (und dem Autor dieses Artikels - um die Perspektive zu verdeutlichen) benannt. Es handelt sich um eine explizite Netzwerkposition, die an den Schnittstellen zwischen Stadtverwaltung und eben jener regionalen Szene wirkt, die wiederum gleichermaßen als Säule wie auch als zu förderndes und auszubauendes Dach des Modells bezeichnet werden kann. Die Kontakte zu Institutionen wie Musikhochschule, Musikschulen oder auch Museen, zu Medien und Akteuren, $\mathrm{zu}$ „Austragungsorten“ von Popkultur wie Clubs und Bars sind es, die das Netzwerk funktionsfähig machen. Des Weiteren sind es die Jugendförderinstitutionen und Organisationen der Stadt aber letztlich auch der öffentlicher Raum, in dem sich Jugend- und Popkultur 
einen Platz zu schaffen sucht. Der Arbeitsbereich wirkt einerseits zwischen Verwaltung und Privatwirtschaft, aber andererseits auch zwischen professioneller Musikwirtschaft und Hobby- bzw. Freizeitkultur. Und schließlich wirkt er nicht zuletzt innerhalb der heterogenen Szene selbst, deren Selbstorganisation gegenüber der (Kultur-)Politik und Stadtverwaltung deutlich verbessert wird.

Die Stelle des Beauftragten ist es, die an dieser Stelle in den Mittelpunkt der Betrachtungen tritt. Nicht nur, weil die Autorenschaft eine detaillierte Binnensicht ermöglicht sondern in der Hauptsache, da Popakademie und Musikpark zwei zwar immens wichtige, aber dennoch in sich selbst weitestgehend autark agierende Institutionen sind, die also solche bewusst Eigeninteressen entwickeln und einen in sich selbst wesentlich komplexeren Studiengangsorganisation, aber nach außen weniger den Gesamtzusammenhang steuernden, Aspekt des Modells verkörpern. Insofern ist die Beauftragtenstelle als „Steuerungseinheit“ für die Verdeutlichung des strategischen kommunalen Popförderhandelns an dieser Stelle besser geeignet, auch wenn die Stelle nicht als eine solche Einheit angelegt ist (zumindest was die Belange der erstgenannten Institutionen betrifft, die absolut eigenverantwortlich aber eben im Verbund handeln).

Die nicht zu vernachlässigende verwaltungsorganisatorische Ansiedlung der Stelle ans Kulturamt der Stadt dient zunächst der Einbeziehung und der besagten „Interessenvertretung“ des Themas in der Stadtverwaltung selbst. Hier wird zudem eine Seite des zweifachen Anspruchs auf Anerkennung deutlich, den Popkultur in der Diskussion vielfach noch immer explizit einfordern muss. Nämlich, als wesentliches (bzw. in einem gewissen Sinne klassisches) Kulturgut wahrgenommen zu werden, das sich in bestimmten Bereichen noch immer den Status als „förderwürdige" Kultur erstreiten muss. Der zweite Aspekt des Ringens dreht sich um die Anerkennung als Wirtschaftsfaktor mit den bekannten Eigenheiten extremer Heterogenität, wirtschaftlicher Kleingliedrigkeit, einem hohen Grad an Image-Wirksamkeit bis hin zu massiver und nach wie vor maßlos unterschätzter Wirkung auf die Standort-Attraktivität insgesamt. Diese Eigenschaft ist es, die eine anhaltende und intensive Zusammenarbeit mit der Wirtschaftsförderung der Stadt begründet aber auch erforderlich macht. Dass der unlängst erfolgte neue Zuschnitt der Mannheimer Dezernatsstruktur zu einer Zusammenlegung u. a. des Wirtschafts- und des Kulturressorts geführt hat, ist alles andere als ein Zufall, sondern versinnbildlicht bereits an der Verwaltungsspitze die strukturelle Arbeitsweise der Popkulturförderung. 
Für den bereits angesprochenen Förderprozess im Sinne der „Wegbereitung" liegen die wesentlichen Potenziale für kommunale Popkulturförderung innerhalb der städtischen Verwaltungsstruktur. Gleichermaßen beschränkt wiederum sind die Handlungsspielräume. Insbesondere dann, wenn es um „Konflikte“ mit Landes- oder Bundesgesetzgebung geht. Zieht man das Thema Lärmschutz als Beispiel heran, wird ohne große Erklärungen deutlich, dass Fragen etwaiger musikalischer Aufführungen in allerlei Zusammenhängen bald nur in engen rechtlichen Korridoren beantwortet werden können.

Die Erwähnung sämtlicher gaststättenrechtlichen Genehmigungsfragen gepaart mit baurechtlichen und feuerpolizeilichen Rahmenbedingungen verdeutlicht den Handlungsrahmen der Fachverwaltungen. Dabei handelt es sich um Verwaltungseinheiten, die sich gemeinhin kaum selbst einen Kultur- (geschweige denn einen Popkultur-) Förderauftrag attestieren würden. Als Grundvoraussetzung für die Bearbeitung des Themenkomplexes (und letztlich für einen Großteil der strukturellen Popkulturförderansätze) muss demnach die strategische Verortung des Themas durch die Verwaltungsspitze gelten. In der Betonung der Querschnittszuständigkeit und der internen Verwaltungskommunikation durch den Oberbürgermeister können viele Ansätze, die für die Entwicklung des Themas Popkultur wesentlich sind, zunächst einmal erkannt und dann deutlich effektiver bearbeitet werden. Aber auch vergleichsweise banale Effekte setzen genau an dieser Stelle an.

Die erfolgreiche Positionierung Mannheims als Musikstadt und ihrer „Organe“ wie der Popakademie haben (vor allem durch die stellenweise immens aufwendigen flankierenden Maßnahmen und Kommunikationsstrategien des Mannheimer Stadtmarketings) zu einer enormen Bewusstseinssteigerung, natürlich auch innerhalb der Stadtverwaltung, geführt und so die Behandlung konkreter Anfragen im Sinne popkulturellen Nutzungen erheblich erleichtert.

Nun werden durch Kommunikationsstrukturen keine Lärmgrenzwerte verändert oder GEMA-Gebühren verringert, aber Genehmigungsverfahren können bei entsprechendem Verständnis z. B. deutlich an die notwendigen Zeitab- und Vorläufe des Veranstaltungswesens angepasst werden. Die erfolgreiche öffentliche Positionierung als „Musikstadt“, „Popstadt“ oder „Ausgehstadt“ ist zwar zunächst nach außen gerichtet, wirkt aber nicht zuletzt auch innerhalb der Verwaltung. Beide Richtungen sind gleichwertige und sich stellenweise gegenseitig bedingende Elemente des gleichen Vorganges und die quer liegenden Verantwortungen sind es, die verdeutlichen, wie sich ein Thema wie „Popkultur“ steuern lässt. 
Das Mannheimer Vorgehen hat exemplarisch gezeigt, dass politische Initiative (des zur Zeit der Planung und Initiation des Modells noch als Kulturdezernent tätigen und heutigen Oberbürgermeisters Dr. Peter Kurz) eine entscheidende Rolle spielen kann und welchen Einfluss ein bis vor wenigen Jahren eindeutig lediglich als Nischenthema wahrgenommener Bereich wie Popkultur auf Stadtentwicklung insgesamt nimmt.

Stadtentwicklung meint hier auch handfeste stadtplanerische Gesichtspunkte. Wie bereits erwähnt hat die absichtsvolle Ansiedlung (bzw. der Neubau) der Popakademie und des Musikparks im ehemaligen Hafenviertel Jungbusch zu einer massiven und messbaren Aufwertung des Quartiers geführt. Die Erschließung des sogenannten Verbindungskanals mittels der Errichtung einer Promenade ist ein weiteres bauliches Beispiel - die sich noch immer intensivierende Zusammenarbeit zwischen den neuen Institutionen und dem sei den 1980er Jahren erfolgreich arbeitenden Quartiersmanagement des ehemaligen Problemkiezes ein anderes.

Auch die weitere Erschließung des Viertels und ehemaliger Brachen durch Konzentration zukünftiger wirtschaftsförderlicher Maßnahmen mit Zielsetzung der Förderung der Kreativwirtschaft wird ausgehend vom Thema „Popkultur“ den Stadtteil und damit die Stadt insgesamt in den nächsten Jahren weiter verändern. Bei all den planerischen Überlegungen sind es jedoch einmal mehr die Menschen, die den Großteil der Veränderung mit sich bringen. Der Wandel vom Problem- zum Studentenviertel ließ und lässt sich im Hafenviertel Jungbusch beinahe täglich beobachten.

Die Tatsache nun, dass immer mehr Städte ihr eigenes kreatives Potenzial entdecken und zu fördern suchen, wird Mannheim in der $\mathrm{Zu}$ kunft Dank der bereits gesammelten Erfahrungen eher zum Vorteil gereichen. Die Erfahrungen, die sich Stadt und Region in der naheliegenden und kommenden Ausweitung der Fördermaßnahmen auf die die Stadt einbettende europäische Metropolregion Rhein-Neckar zunutze machen möchten, haben auch dazu geführt, dass Mannheim selbst zu einem Ansprechpartner für andere Städte/Regionen und Organisationen geworden ist.

Diese zusätzlichen Anknüpfungspunkte sind es, die das Popkulturnetzwerk durch ständigen Austausch entscheidend bereichern. So sind es die vorhandenen Strukturen der Stadt, die den Akteuren schon heute im europäischen und nationalen Umfeld regen Austausch von Informationen und Kontakten ermöglichen (bspw. auf der Ebene von konkreten Festivalpartnerschaften). Die Zielsetzung lautet, als „Kompetenzzentrum“ auch international wahrgenommen zu werden. Nun ist „Kompe- 
tenz" in Popkulturfragen ein stellenweise nur diffus zu zeichnender Begriff. Zum einen kann man darunter selbstverständlich die fundierte und der Schnelllebigkeit sowie dem radikalen Umbruch des Musikgeschäftes angepasste Ausbildung an der Popakademie verstehen.

Darüber hinaus mögen es schwer messbare Bereiche wie innovative Ausgeh- und Konzertkultur sein, die einen wesentlichen Teil zur Wahrnehmung als in diesem Bereich „kompetente“ Stadt“ beitragen. Das zuvor als ,popkulturelles Grundrauschen“ bezeichnete Klima der Stadt, das wie nebenbei von einer regionalen Wirtschafts- und Bildungsstandortstruktur flankiert wird, die mit IT-Technolgie Weltmarktführern einerseits und einer bundesweit anerkannten Hochschule für Gestaltung aufwarten kann, ist Grundlage der begründeten Hoffnung, dass das Mannheimer Modell auch weiterhin als Erfolgsmodell für Entwicklung, Austausch, Innovation und letztlich (und vor allem) für viel lebendige und sich ständig selbst erneuernde gegenwärtige Popkultur sorgen wird. 



\section{Design Reaktor Berlin: Ingredienzien einer Prozessgestaltung}

\section{JUDITH SENG}

Der Design Reaktor Berlin ${ }^{1}$ beschreibt einen definierten Raum, der mit verschiedenen Ingredienzien angereichert wird, um experimentelle Reaktionen zu erzeugen. Übergeordnetes Ziel dieses ersten Reaktors war der Anstoß zukunftsweisender Entwicklungsstrategien für postindustrielle Standorte wie Berlin. Die Aufgabe bestand darin, das Zusammenspiel kreativer und produzierender Milieus mit einem gemeinsamen Produktentwicklungsprozess neu zu überdenken und letztlich zu verbinden.

Das angewandte Forschungsprojekt Design Reaktor Berlin wurde Anfang 2007 an der Universität der Künste Berlin initiiert. Den Auftakt bildete ein zweiwöchiger Workshop-Cluster zur Ideenfindung, gefolgt von einer Jury, die die vielversprechendsten Ideen auswählte. Nach einer intensiven zwölfwöchigen Entwicklungsphase bezeugten 52 Prototypen, sechs Urheberrechtsanmeldungen und viele Bilder das dynamische und fruchtbare Geschehen. Die besten Prototypen wurden weiterentwickelt und individuell verwertet: von der Kleinserie, die bereits nach sechs Monaten im Museumsshop des Guggenheim Berlin verkauft wurde, über Ausgründungen bis hin zu Kooperationen mit Partnern, die Kontakte zur Industrie knüpfen. Weiterführende Initiativen zur Etablierung einer experimentellen Entwicklungs- und Verwertungsplattform zwi-

1 Der Design Reaktor Berlin wurde von Axel Kufus, Marc Piesbergen, Joachim Schirrmacher und Judith Seng an der Universität der Künste Berlin geleitet und vom Berliner Senat für Wirtschaft, Technologie und Frauen aus Mitteln des Europäischen Fonds für regionale Entwicklung (EFRE) kofinanziert. www.design-reaktor.de 
schen Forschung und Markt sind ein weiteres Ergebnis des knapp zweijährigen Projekts. Weniger sichtbar und messbar sind die vielfältigen und langwierigen Prozesse, die anhand der Produktentwicklung angestoßen wurden; die persönlichen Begegnungen und Erfahrungen, der Wissens- und Kompetenzaustausch, die neuen Perspektiven und Impulse, die weit über das spezifische Projekt hinaus wirken. Trotz der Schwierigkeit, diese flüchtigen und unsichtbaren Ergebnisse als Wert begreifbar zu machen, haben alle diese Ingredienzien wesentlich zum Erfolg der Reaktor-Prozesse beigetragen.

\section{Produkte als Vehikel}

Die große Stärke eines Produkts liegt in seiner Körperlichkeit. Ein Objekt besitzt einen Ausdruck und kann einen Nutzen haben; es ist immer fassbar, meist herstellbar und manchmal verkaufbar. Diese Formgebung einer Idee, ihre „Geburt“ als Prototyp, zählt zu den eindrucksvollsten Kompetenzen der Designdisziplin. Als greifbares Objekt kann die Idee plötzlich begehrt oder gehasst, erprobt und sogar besessen werden. Vor allem aber scheinen Konsumenten, Vermarkter, Designer, Wissenschaftler und Journalisten diese fassbare Sprache gleichermaßen zu verstehen. Übertragen auf die Produkte des Design Reaktors Berlin sollten sie nicht nur ihre emotionale bis funktionale Alltagsaufgabe erfüllen, sondern gleichzeitig als Mittler zwischen verschiedenen Gestaltungsdisziplinen und Berliner Produktionsbetrieben, zwischen Hochschule und Markt agieren, um so die unterschiedlichen Kompetenzen, Rhythmen, Sprachen und Werte in einem gemeinsamen Vorhaben zusammenzubringen.

Im Design Reaktor Berlin provozierte die zeitliche Verdichtung und das disziplinäre Zusammenrücken eine inhaltliche und räumliche Erweiterung der beteiligten Institutionen. Schon während der ersten Ideenfindung wurden unterschiedlichste Akteure aus Vermarktung, Produktion und Recht involviert, um die Entwicklung von Anbeginn aus diversen Perspektiven mitzugestalten.

Aus einer sozialen Perspektive auf die Produktentwicklungsprozesse sind die entstandenen Produkte daher in erster Linie Vehikel für Kooperationsprozesse zwischen Disziplinen, Hochschule und Markt. Nutzen und Vermarktung stehen demnach zwar an zweiter Stelle, sind aber gleichzeitig unverzichtbar, um den Austausch zwischen den Milieus zu provozieren. Sie stellen den eigentlichen Anreiz für die Entwicklung vitaler Eigendynamiken und den Aufbau von Kompetenznetzwerken zwischen Patentrecht, Vermarktung, Produktion und Gestaltung. 


\section{Open Briefing}

Ein Plan beschreibt meist, was aus der aktuellen Perspektive schon vorstellbar ist. In der Praxis zeigte sich, wie viel mehr entstehen kann, wenn ein Plan nur minimale Strukturen vorgibt und sich erst mit Verlauf des Projekts vollständig entwickelt. Ausgangspunkt des Design Reaktors Berlin war eine kaum zweiseitige Vorhabensbeschreibung mit kurzer Planungsphase, in der grundlegende Meilensteine definiert und Teilnehmer gewonnen wurden. Erst im eigentlichen Projektverlauf entwickelte sich der Design Reaktor Berlin zu dem, was er heute ist, denn im offenen Prozess können sich ungeahnte Potenziale entfalten: Erwartungen können unterlaufen, aber vor allem auch übertroffen werden.

Diese Unschärfe in der Projektsteuerung wurde im Design Reaktor Berlin unter dem Begriff „Open Briefing“ diskutiert, als eine Arbeitsweise die die Identifikation mit dem Projekt erhöht da Spielraum für individuelle Entwicklungen entstehen kann. Diese Erfahrungen offenbaren spannende Möglichkeiten, aber auch neue Herausforderungen, die dynamische und komplexe Prozesse an ihre Steuerung stellen.

\section{Heterogenes Netzwerk}

Das Besondere am Design Reaktor Berlin? Hier trafen Menschen zusammen, die sonst kaum miteinander in Berührung gekommen wären. Interessante Heterogenität war durch die sorgfältige Auswahl der Projektpartner garantiert: Von insgesamt 75 persönlich angesprochenen, mittelständischen Kleinunternehmen aus Berlin konnten 55 zur Teilnahme am Design Reaktor Berlin gewonnen werden. Auch innerhalb der Universität der Künste Berlin beteiligten sich fünf verschiedene Fachrichtungen der Fakultät Gestaltung, ergänzt durch zahlreiche externe Experten. All diese Projektteilnehmer haben den Design Reaktor Berlin als tatkräftige Akteure, konstruktive Beobachter, Berater oder Unterstützer entscheidend definiert und mitentwickelt.

\section{Verbindende Räume}

Wie sieht ein Ort, eine Plattform aus, die eine heterogene Mischung von Disziplinen, Branchen und Institutionen temporär zusammenbringt? Wie können die spezifischen Arbeitsweisen, Sprachen und Werte einander verstärken? Gleich zu Beginn des Projekts wurde über einen Kooperationsvertrag, der allen Urhebern eine Beteiligung am möglichen Profit zusichert, ein „Vertrauensraum“ definiert, um Prozesse mit offenem Ausgang zu fördern. Auf anderer Ebene lieferte das Corporate Design 
des Design Reaktors einen übergreifenden „Identifikationsraum“. Ganz im Sinne des Projekts entstand das Corporate Design parallel zu den Produktentwicklungen und verkörpert als Abbild der entstehenden Projektidentität den institutions- und bereichsübergreifenden, symbolischen Projektraum.

Die eigentlichen, physischen Projekträume wurden hingegen nur temporär bespielt. Zwei „Open Spaces“‘2 an der Universität der Künste dienten als Plattform für kleinere und größere Zusammenkünfte, ein kleines Design Reaktor-Büro als kontinuierliche Anlaufstelle für das weit verstreute Netzwerk. Die meisten der 180 Beteiligten arbeiteten in ihren eigenen Werkstätten und Büros in ganz Berlin.

\section{Öffnende Räume}

Durch konkretes Handeln und sichtbare Ergebnisse hat das Projekt große Resonanz erzeugt. Gleichzeitig wirft diese praxisorientierte Arbeitsweise viele übergreifende Fragen auf, die über das spezifische Projekt hinaus von Bedeutung sind. Denn was bedeutet es, wenn eine Kunsthochschule Patente anmeldet? Oder sollten angewandte künstlerische Strategien vielmehr das Diskussionsfeld zwischen „Open Source“ und „Intellectual Property Rights“ erweitern? Denn wer ist bei dieser multiperspektivischen Produktentwicklung überhaupt der Urheber? Und welche Auswirkungen hat dies auf unseren Umgang mit Autorenschaft?

In der Praxis wird deutlich, welche Chancen und Herausforderungen bei der Organisation komplexer Prozesse auftreten, wenn sich die Strukturen erst durch das Projekt herausbilden. Wie also könnten Handlungsstrategien für eine integrative Planung und Umsetzung aussehen, um von Top-down und Bottom-up Steuerung zu profitieren?

Fragestellungen dieser Art könnten zukünftig in entsprechenden „Denkräumen“ vertieft werden, die den praktischen Prozess begleiten. Es könnte ein Zusammenspiel aus schnellem, wirksamem Handeln und übergeordneter Reflexion entstehen, das eine nachhaltige, vielschichtige Wirkung erzeugt. Der Design Reaktor - als definierter Raum für den praktischen Anstoß experimenteller Entwicklungen - würde so zum umfassenden Prototyp für exemplarische Handlungsszenarien, um die verschiedenen Ingredienzien einer Projektgestaltung - wie Produkte, Prozesse, Räume und Denkräume - iterativ zu gestalten.

2 Darunter verstehen wir unbesetzte Räume, die immer wieder neu bespielt werden können und nicht von einer Gruppe oder Person dominiert werden. 
Neue Institutionen 



\title{
Schnittstellenkulturen - Hybride Akteure, Patchworkökonomien, intermediäre Institutionen
}

\author{
INGA WELLMANN
}

Globalisierungs- und digitalisierungsbedingte Dynamiken führen zu einer neuen kulturellen Komplexität, die ein Denken und Handeln in festgelegten Kategorien und Disziplinen kaum noch zulässt. Die Kultur der Schnittstelle und die ständige Vermischung vormals getrennter Einheiten markiert gleichsam das Selbstverständnis der Netzwerkgesellschaft. Die Art und Weise, wie produziert und konsumiert, kommuniziert und interagiert wird, hat sich durch die Massennutzung neuer Informationstechnologien grundlegend verändert. Entstanden sind Kulturen der Querverweise, Weiterverarbeitung und Wiederaneignung, in denen die Grenzen der Zuständigkeiten, Kompetenzen und des intellektuellen Eigentums verschwimmen.

Merkmale einer solchen Komplexität lassen sich heute vielen Lebens- und Wirkungsbereichen zuschreiben: Ständiger Wandel, unsichere Verhältnisse, ein hoher Grad von Vernetzung und Interdependenz, Unschärfe und Vielschichtigkeit kennzeichnen nicht nur die kreativen Ökonomien, sondern bilden neue Parameter einer weit gehenden Transformation in Politik, Wirtschaft und Gesellschaft. Schnittstellenkompetenz wird dabei zum unabdingbaren Gut des postindustriellen Wissenszeitalters, nicht nur, wenn es um die Frage der Steuerbarkeit kreativer Ökonomien geht. Eine intelligente Gestaltung der Schnittstellen zwischen den verschiedenen Funktionssystemen Wirtschaft, Wissenschaft, Kunst und öffentlicher Sektor dürfte Wachstum und Innovation in großem Ausmaß stimulieren. 
Ziel dieses Beitrags ist es, die Dimension des Intermediären und Hybriden sowohl auf Akteurs- als auch auf Institutionsebene näher zu betrachten und $\mathrm{zu}$ überprüfen, ob diese als Ansatzpunkt für eine mögliche (Selbst-)Steuerung kreativer Ökonomien geeignet wäre. Dazu wird im Folgenden ein Einblick in die schnittstellenorientierte Haltung vieler Kreativarbeiter angeboten, in ihre überwiegend von Mischtechniken geprägten Strategien und patchworkartigen Handlungsarenen sowie die dieser Logiken entspringenden neuen Organisationsformen. Abschließend wird die Frage nach dem Bedarf neuer intermediärer Institutionen gestellt, die mit dem Ziel eines strategisch informierten Schnittstellenmanagements die Brücke zwischen (selbst-)organisierten Akteursnetzwerken und etablierten kultur- und wirtschaftspolitischen Strukturen zu schlagen vermögen.

\section{Kultur der Schnittstelle im Kontext von Hybridisierung und Hyperkulturalität}

Die teils bewusste, teils (noch) unbewusste Schnittstellengestaltung bildet einen zentralen Aspekt im Wandlungsprozess heutiger Arbeitskulturen. Sie geht über die in den Wissenschaften und Künsten in den letzten Jahrzehnten immer intensiver praktizierte interdisziplinäre Dimension hinaus. Vielmehr entstehen auf allen Ebenen der Gesellschaft neue Kooperationsformen, die oft sektorenübergreifend angelegt sind und das Selbstverständnis etablierter Denkmuster und Organisationsstrukturen in Frage stellen. Bisherige Deutungsmuster geraten an ihre Grenzen, wenn es um die Beschreibung neuer hybrider und zukunftsweisender Formen geht.

Eine Hybridisierung der Formen zeichnet sich auf Mikro- und Makroebene ab: Ob im Profil eines Einzelakteurs oder in der Entstehung einer neuen Institution, ob in der politischen Ökonomie oder in der raumzeitlichen Dimension postindustrieller Symbolproduktion - Wertschöpfung und Wachstum der globalen, multikulturellen Netzwerkgesellschaft vollziehen sich in der Verdichtung und Verschmelzung vormals getrennter Strukturen: „Hybrid ist alles, was sich einer Vermischung von Traditionslinien oder von Signifikantenketten verdankt, was unterschiedliche Diskurse und Technologien verknüpft, was durch Techniken der collage, des samplings, des Bastelns zustandegekommen ist“" (Bronfen 1997). Die kulturelle Dynamik der Gegenwart wird von dem koreanischen Philosophen Byung-Chul Han auch als eine entgrenzte Hyperkultur beschrieben: 
Der Globalisierungsprozess wirkt akkumulierend und verdichtend. Heterogene kulturelle Inhalte drängen sich in einem Nebeneinander. Kulturelle Räume überlagern und durchdringen sich. Die Entgrenzung gilt auch der Zeit. Ins Nebeneinander des Verschiedenen werden nicht nur unterschiedliche Orte, sondern auch unterschiedliche Zeiträume entfernt. Nicht das Gefühl des Trans, Inter- oder Multi-, sondern das des Hyper- gibt präziser die Räumlichkeit der heutigen Kultur wieder. [...] Nicht Grenzen, sondern Links und Vernetzungen organisieren den Hyperraum der Kultur. (Han 2005)

Dieser relationale Charakter ist auch bei den kreativen Ökonomien wesensimmanent, wobei diese sich zwischen Polaritäten bewegen: Zum einen sind sie gekennzeichnet durch die Verbindung vieler heterogener Elemente, zum anderen aber auch durch Zerstreuung und Entortung. Was bedeutet das für die im kreativen Kontext arbeitenden Einzelakteure?

\section{Unvollständige Akteure in transversalen Projektwelten}

Der kreative Akteur präsentiert sich zunehmend als Collage verschiedener Identitäten und Kompetenzen. Während der amerikanische Soziologe Richard Sennett dem ,flexiblen Menschen“ im Jahre 1998 noch eine gewisse Ohnmacht, Orientierungslosigkeit und Fremdbestimmung zuschrieb - um in den komplexen Interessensgefügen des Kapitalismus zu funktionieren, sei das Individuum gezwungen, sich entsprechend vielseitig aufzustellen, mit der Folge instabiler zwischenmenschlicher Beziehungen und Vereinzelung (Sennett 1998) -, so wird dieses Phänomen heute durch die Akteure selbst aktiv mitbestimmt und gestaltet. Kreative Selbst- und Einzelunternehmer gelten inzwischen als treibende Kraft der ideen- und wissensbasierten Ökonomien.

Der ehemalige Berater der Blair-Regierung Charles Leadbeater stellt diese auch als „Independents“ (Leadbeater 1999) vor. Diese Independents passen in keine festen Kategorien und agieren oft als Mikrounternehmer zugleich in der Rolle des Schöpfers, Produzenten, Kaufmann und Marketingmenschen in einer Person:

Although some are ambitious entrepreneurs, many want their businesses to stay small because they want to retain their independence and their focus on their creativity. Yet that does not mean they see themselves as artists who deserve public subsidy. They want to make their own way in the market. They 
have few tangible assets other than a couple of computers [...]. Their main assets are their creativity, skill, ingenuity and imagination.

Uneindeutigkeit und Vielfalt kennzeichnen nicht nur die rahmengebende Wirtschaftskultur, sondern auch die Tätigkeitsprofile der einzelnen Akteure. Die Rede ist bewusst von „Tätigkeitsprofil“ und nicht von „Berufsprofil“", da auch der Begriff der Arbeit einem grundlegenden Wandel unterliegt: Arbeit ist nicht mehr die zeitlich klar strukturierte und dezidiert fremdbestimmte Ausübung eines fest umrissenen Berufes, sondern vielmehr eigenmotiviertes, selbständiges Denken und Handeln entlang individueller Interessensfelder (siehe u. a. Friebe/Lobo 2006).

Die Angehörigen der kreativen Milieus sind miteinander vernetzte, dennoch hochindividualisierte Lebensgestalter und Sinnproduzenten (Lotter 2009) mit oftmals ausgeprägter Schnittstellenorientierung. So tauchen neue Tätigkeitsprofile in unterschiedlichen synthetischen Begriffsgewändern auf: Vom Culturepreneur (Lange 2007) über den Cultural Catalyst, Cultural Broker und Cultural Hacker (Liebl/Düllo 2005) hin zum digitalen Bohemien (Friebe/Lobo 2006), Bricoleur (LéviStrauss 1962; Friebe/Ramge 2008) und Social Surfer (Wellmann 2007). Was alle Profile kennzeichnet, ist ihre Transversalität und Unvollständigkeit. Transversalität insofern, als dass sie über transdisziplinäre Kompetenzen verfügen und diese je nach Bedarf einsetzen; Unvollständigkeit (Priddat 2005a) indem sie sich über den Bezug zu anderen definieren und erst in der Verbindung zu Netzwerkpartnern ihr Potenzial entfalten.

Allen gemein ist die Broker-, bzw. Agentenhaftigkeit: Sie verorten sich an den Schnittstellen verschiedener Disziplinen und sozialer Welten und schöpfen über eine entsprechende Vernetzung den entsprechenden Mehrwert. Sie sind Broker sozialen Kapitals und damit Übersetzer, Vermittler, Mediatoren in einer Ökonomie der Aufmerksamkeit und Anerkennung. Sie besetzen leere Räume und „structural holes“, von denen aus neue Ideen und Gelegenheiten für Innovation schnell zu erfassen sind (Burt 2005). Ihr Wirken ist projektförmig und damit auf ständige Transformation und Kooperation angelegt. Sie begegnen damit den Strukturveränderungen des heutigen Kapitalismus, in welchem heterarchische, oft netzwerkbasierte Organisationsformen die hierarchischen, monolithischen Strukturen des Industriezeitalters verdrängen, und werden daher als Schnittstellenakteure bezeichnet.

Boltanski und Chiapello beschreiben in ihrem Buch „Der neue Geist des Kapitalismus“ die moderne Netzwerkgesellschaft als ,projektbasierte polis“ und stellen damit den zeitlich begrenzten Projektcharakter des sozialen Raumes an der Schnittstelle von Politik und Gesellschaft he- 
raus. Jede Beziehung innerhalb komplexer Netzwerkstrukturen werde demnach als Projekt be- und dadurch ständig neu verhandelt. Jederzeit können neue Kooperationen gebildet werden. Als Ordnungsmerkmale der Netzwerkgesellschaft benennen sie Flexibilität und Konnexivität (Boltanski/Chiapello 2003).

Nun existieren Projekte nicht erst seit Beginn des postfordistischen Wissenszeitalters. Jedes zeitlich begrenzte Vorhaben kann als Projekt bezeichnet werden. Der Begriff des „Projektes“ taucht jedoch erst seit dem 17. Jahrhundert im deutschen Sprachgebrauch auf - und zwar immer dort, wo durch eine Krise neue Lösungen und Handlungsalternativen gefragt sind: „Die Konjunktur der Projektemacherei ist eine Antwort auf die Krise des Staats und seiner unsicheren (ökonomischen) Situation“ (Krajewski 2004). Während der „Projektemacher“ in früheren Zeiten jedoch gerade aufgrund seiner unsteten und damit nach damaligen Wertemustern wenig Vertrauen erweckenden Arbeitspraktiken eher marginalisiert oder als „Aventurier“ und „komische Figur“ (Stanitzek 2004) deklassiert wurde, sind die heutigen Projektemacher im Zentrum der Gesellschaft angekommen.

Laut Boltanksi/Chiapello ist dies ein wichtiges Ergebnis des reformerischen Impulses der 1968er Bewegung, der nicht nur kulturelle Formen der Emanzipation ermöglichte, sondern auch für das Entstehen einer „,intelligenteren“ Managementpraxis sorgte. Inzwischen gehören Projektemacher mit ihrer Schnittstellenkompetenz zu den wichtigsten Motoren wirtschaftlicher und gesellschaftlicher Entwicklung. Dank ihrer kosmopolitischen Orientierung und ihrer Fähigkeit, mit instabilen Verhältnissen und möglichem Scheitern souverän umzugehen, entwickeln sie jene Strategien, die nach dem Ende des Industriekapitalismus voraussichtlich überlebenswichtig sind.

\section{Strategien kollektiver Selbstermächtigung}

Charles Leadbeater postuliert in seinem 2008 erschienenen und im Open Source-Verfahren geschriebenen Buch „WE-THINK: Mass innovation, not mass production“ den Beginn einer neuen Wirtschafts- und Gesellschaftsordnung, die auf kollektiver und kollaborativer Kreativität beruht - und damit auf einer „Produktivität der Unvollkommenheit“ (Krajewski 2004). Der experimentelle Charakter und der situationsorientierte Umgang mit (Nicht-)Wissen kennzeichnet diese fragmentierten Ordnungen (siehe auch Einleitungstext „Fragmentierte Ordnungen“ in diesem Buch). Nach kompositorischen Prinzipien werden in den Transformations- und Teilhabekulturen der heutigen Netzwerkgesellschaft Ideen und 
Wissen verbreitet, neu zusammengestellt, weiterverwertet, und wieder verbreitet. Es sind Techniken des Samplings und der Bricolage, die sich die Akteure der kreativen Ökonomien aneignen, um der Komplexität heutiger Arbeitszusammenhänge Sinn und Mehrwert abzugewinnen. Auch Guerilla-Techniken wie Camouflage, spontane Präsenz und Widerstand kommen zum Einsatz und unterstützen die Bewegung einer kollektiven Intelligenz, die sich in stetig wechselnden Rahmenbedingungen entsprechend flexibel aufzustellen weiß. Eine ständige Reproduktion von Ordnung vollzieht sich, Störungen sind fester Bestandteil dieses Prozesses, und mit Hilfe moderner Web 2.0-Technologien werden jenseits sichtbarer öffentlicher Ordnungen eigene Subsysteme entwickelt.

Ähnlich wie die Situationistische Internationale rund um Guy Debord in den 1960er Jahren den öffentlichen Raum mit ihren Konzepten und Aktionen infiltrierte, eignen sich viele der in diesen soziotechnologischen Zusammenhängen agierenden Akteure und Netzwerke virtuos den virtuellen Raum an, u. a. mit Hilfe des Cultural Hackings als „Kulturtechnik, die versucht, Systeme zu decodieren und in sie transformativ einzudringen“" (Borries 2008; siehe auch Liebl/Düllo 2005). Yana Milev beschreibt diese Gruppierungen, die oftmals in prekarisierten Zusammenhängen agieren und deren gleichzeitige Selbstermächtigung auf einer strategischen Ausschöpfung technologischer Möglichkeiten fußt, als „Freelance-Mobility“, „Outcast-Eliten“ und „Subraummacht" (Milev 2008).

Gerade in den heutigen, nach Authentizität und Unterscheidbarkeit strebenden Unterhaltungsindustrien aber findet Randständiges und Subkulturelles schnell den Weg in die allgemeine Wertschöpfungskette. Die Peripherie rückt ins Zentrum. Gleichzeitig werden Wirtschaftspraktiken in der Kunst neu umgesetzt. Die Berührungspunkte von Kunst und Wirtschaft liegen damit längst jenseits von Kunstmärkten oder unternehmenspolitische Maßnahmen wie Kultursponsoring. „Raffinierter überleben“ (Brellochs/Schrat 2005) bedeutet einmal mehr, die Grenzen der Disziplinen durchlässig zu machen und Wechselwirkungen zuzulassen.

Dies zeigt sich auch in der Managementausbildung, die zunehmend multidisziplinär gestaltet wird (bspw. die Zeppelin University mit dem Claim „Bridging Business, Culture and Politics“). Dass dabei keine Kompromisskultur im Vordergrund steht, sondern vielmehr eine Kultur des produktiven Dissenz, ist ein weiteres Merkmal einer neuen Wirtschaftskultur, die ihre Potenziale in Unterschieden sieht: „Awareness of uncommon ground is awareness of a possible relation across difference“ (Putnam 2007). Es scheint, als würde Deleuzes Ontologie von „Differenz und Wiederholung“ (Deleuze 1968) zum Wesen einer neuen Kapi- 
talismuskultur: Das ,aktive Herstellen von Differenz“ wird zur Grundbedingung, Abweichung wird zur Norm (Osten 2003).

\section{Märkte der Vielfalt, Produkte der Transformation}

Das Randständige und individuell Modifizierte kennzeichnet auch die neuen Märkte der kreativen Ökonomien: Es sind Märkte der Vielfalt und Nischen (Lotter 2009). Die massive Reduktion von Transaktionskosten durch das Internet ermöglicht es immer mehr kreativen Individuen, zu Kleinstunternehmern zu werden und ihre eigenen, stark nischenorientierten Produkte und Marken zu Markte zu tragen (Friebe/Ramge 2008). Die Unvollständigkeit und Transversalität der Akteure und Märkte der kreativen Ökonomien gilt wechselseitig auch für ihre Produkte, die als „unfertige Transformationsgüter“ (Priddat 2009) alle Akteure, mit denen sie in Berührung kommen, verändern - also ihre Nutzer, Produzenten, Konsumenten.

Als weitere Variante der Prosumption, in der der Konsument selbst als Produzent in die Produktgestaltung miteinbezogen wird (diese Nutzbarmachung der individuellen Ressourcen eines Kunden zur Produktfertigstellung wird inzwischen auch als Gegentrend zur Dienstleistungsgesellschaft identifiziert: der Kunde arbeitet für die Wirtschaft, bspw. IKEA) sind diese Transformationsgüter Produkte mit hohem symbolischen Wert, deren Wertschöpfung erst im Prozess erfolgt und die durch ihren Sinngehalt und in ihrer Funktion als kultureller Erkenntnisträger den Benutzer selbst verändern. So prägt ein Buch oder ein Film den jeweiligen Konsumenten auf andere Weise, als es etwa ein mass customized Nike Turnschuh oder ein selbst zusammengesetztes IKEA-Regal vermag (Priddat 2009).

Der US-amerikanische Rechtsanwalt und Open Source-Aktivist Lawrence Lessig, bekannt geworden unter anderem durch sein Engagement für Creative Commons, spricht im Kontext einer von Transformationsgütern und kollektiver Kreativität geprägten Wirtschaft von einer „Hybridökonomie“, in der die Grenzen der Disziplinen durch Nutzer modifiziert werden. Andere, stärker aus der sozialen Sphäre resultierenden Formen der Wertschöpfung werden hervorgebracht und in komplexen, arbeitsteiligen Gemeinschaftsprozessen weiterentwickelt. Open Source als Organisationsprinzip ermöglicht eine Schwarmintelligenz, in der Solidarität und Wettbewerb Hand in Hand gehen.

Schwarm und Multitude sind in diesem Kontext Begriffe einer Beschreibung neuer Vergemeinschaftungsformen bei gleichzeitigem Erhalt 
der individuellen Autonomie (Virno 2005; Hardt/Negri 2003 und 2004). Laut Priddat scheint es ,eine angemessene Form moderner Sozialität zu sein: Konkurrenz und Kooperation parallel oder sequentiell oder zyklisch zu betreiben - in Projekten lose gekoppelt, immer disponiert für etwas anderes (ohne es mit Notwendigkeit anders machen zu müssen)“. Open Source wird hier verstanden als ,komplexe mögliche Welt, in der Dinge gekreuzt werden können, die woanders noch weit auseinander liegen" (Priddat 2009).

Als bildhaftes Modell steht der Basar Pate, der sich aus vielen beweglichen und miteinander in ständigen Austauschprozessen stehenden Kleinstakteuren konstituiert. Er ersetzt, so der amerikanische Autor und Hacker Eric S. Raymond, die starre, Ehrfurcht gebietende Kathedrale (Raymond 1997). Der Einzelne organisiert sich in unterschiedlichen dynamischen Netzwerkkonstellationen, arbeitet dabei unverwechselbare und damit Glaubwürdigkeit und Wiedererkennungswert herstellende Nischenkompetenzen heraus.

In diesen Patchworkökonomien verbinden sich viele autonome Einheiten zu einem synergetischen Gefüge, welches vorindustriellen Formen „munizipaler Selbstorganisation“ gleicht: „Die zukünftige Landschaft der Organisationen wird diesem Flickenteppich wieder stärker ähneln. Sie wird eine Textur aus Clustern und vernetzten kleinen Einheiten aufweisen, die abseits der traditionellen industriellen Zentren liegen und deren Verbindungslinien quer zu den Logistikströmen der Massenproduktion verlaufen“ (Friebe/Ramge 2008).

\section{Schnittstellenorganisation und die Emergenz neuer (alter) Institutionen}

Das Institutionengefüge in den wissensbasierten, kreativen Ökonomien verändert sich in demselben Maße, wie es die Akteure, Märkte und Produkte tun - und auch, weil es sich verändern muss. Nur wenige etablierte Institutionen sind heute in der Lage, zeitgemäß mit den Transformationen der letzten Jahrzehnte umzugehen. Es bedarf neuer Institutionen, die auf die sozio-technologischen Implikationen einer Netzwerkgesellschaft zu reagieren wissen - einer Gesellschaft, die stärker von der Repräsentation auf Kooperation umstellen wird und die Grundwerte der Wikinomics verinnerlicht haben: Peering, eine neue Offenheit, eine Kultur des Teilens sowie globales Handeln (Tapscott/Williams 2007).

Auffällig in diesem Kontext ist das Wiedererstarken alter, teilweise vorindustrieller Organisationsformen. Bereits die Idee der Creative Commons fußt auf der Idee der Allmende als alter Rechtsform gemein- 
schaftlichen Eigentums. Wo früher innerhalb einer im Besitztum der Dorfgemeinschaft stehenden Gemarkung die Kühe weideten, werden heute - begleitet von einem stufenförmig aufgebauten, vom Urheber selbst auf seine Werke applizierbaren Lizenzsystem - kulturelle bzw. intellektuelle Ressourcen der Allgemeinheit zur Verfügung gestellt. Und auch alte Prinzipien der Gilde werden heutzutage neu interpretiert und an die Logik multikultureller, transdisziplinärer Zusammenhänge angepasst. Der Schutz und die Förderung gemeinsamer Interessen, die im Mittelalter zur Gründung von Gilden geführt hat, stehen beispielsweise auch bei der Amsterdamer Mediagilde im Vordergrund.

Hier lautet das gemeinsame Interesse, eine maximale Realisierung des Innovationspotenzials zu erreichen, das in den modernen Informations- und Kommunikationstechnologien sowie in den medienorientierten kreativen Ökonomien liegt. Direktes Lernen von einem Meister (Experten) und das Arbeiten in kleinen Gruppen in optimalem Umfeld soll den praxisorientierten Austausch von Erfahrung und Wissen ermöglichen, so der Direktor Andrew Bullen.

Er sieht die Institution als neue Form der ,post-education education“ und damit als wichtigen Inkubator für die Zeit nach der Ausbildung, aber noch vor der Start-up-Phase: ,pre-seed“ (Bullen/Szita 2007). Die Institution funktioniert in der Logik sozio-technologischer Netzwerke und positioniert sich als Innovationsplattform und „kreatives Ökosystem“ an der Schnittstelle zwischen Ausbildung und Markt, zwischen Wissenschaft und Wirtschaft, zwischen öffentlichem und privatem Sektor.

Ein weiteres Gemeinschaftsmodell, das heute gerade im kreativen Bereich wieder an Zuspruch gewinnt, ist die eingetragene Genossenschaft. So bietet die Berliner self eG (Social Entrepreneurship \& Leadership Foundation) ihren Mitgliedern einerseits eine rechtliche Selbständigkeit, andrerseits gleichzeitig auch die „Vorteile eines großen Unternehmens wie Kostenvorteile, Interessenvertretung und erhöhte Sichtbarkeit nach außen“.

Das „Ermöglichen sozialer Innovation“, das der Institution als wichtigste Zielsetzung eingeschrieben ist, unterstreicht einmal mehr die Abkehr von rein finanziell gewinnorientierten Modellen des Wirtschaftens hin zu einer Ökonomie, die ihre Wertschöpfung aus einer nachhaltigen Maximierung gesellschaftlichen Nutzens zieht. Gleichzeitig hat sich die Genossenschaft über die Einrichtung des Co-working Space selfHUB, der „das Beste von Gründerzentren, Bibliothek, Cafe und Bürolounge“ vereinen soll, an ein weltweites Netz von selfHUBs als Zentren gesellschaftlicher Innovation in London, Brüssel, Rotterdam, Johannesburg, Mumbai und Sao Paulo angeschlossen (www.self-germany.de). 


\section{Organisierte Netzwerke versus vernetzte Organisationen}

Einen Vorschlag für eine Definition neuer Institutionsformen im Medienzeitalter macht der australische Medientheoretiker Ned Rossiter, in dem er den Begriff des „organisierten Netzwerks“ einführt. Er beschreibt damit die emergenten institutionellen Formen, die sich durch Transdisziplinarität, Distributivität und Kooperation auszeichnen. Institutionen verstehen sich seit jeher in der Funktion, soziale Verbindungen zu organisieren.

Organisierte Netzwerke jedoch unterscheiden sich dahingehend von den modernen ,vernetzten Organisationen“ - also Gewerkschaften und jene Unternehmen, die sich vertikal organisieren und repräsentationalen Logiken folgen - , als dass sie sich explizit an den Kooperations- und Verteilungspraktiken der digitalen Kommunikationskultur mit ihren Social Networks und Open Source Praktiken orientieren (Rossiter 2006). Damit lässt sich auch gleich eine klare Zuordnung vollziehen: Das emergente ,organisierte Netzwerk“ ist eng an die heutige Medienkultur gebunden, während die ,vernetzten Organisationen“" noch eine ältere institutionelle Form darstellen, die sich lange vor dem Einzug moderner und allgemein verfügbarer Informationstechnologien begründet hat. Unterschiedliche Haltungen begründen sich beispielsweise im Umgang mit intellektuellem Eigentum: Während die etablierten Institutionen meist ihr ganzes Rechts- und Handelsgefüge auf die strikt regulierte Verwertung intellektuellen Eigentums aufgebaut haben, operieren Netzwerke des Medienzeitalters meist eher in der Open Source-Logik, d. h. in der freien Zirkulation und Weiterverarbeitung von Ideen.

Gekennzeichnet sind diese neuen (selbst-)organisierten Strukturen durch ein hohes Maß an Informalität, Fragmentierung, Adaptibilität, lokalem Bezug und Transition als Dauerzustand (Bradley et al. 2006). Diese neuen Formen der projektorientierten (Netzwerk-)Organisation werden mit Verweis auf die klassische Markt/Organisations-Dichotomie der Transaktionskostenökonomie (Williamson 1975) auch als Hybride bezeichnet (Priddat 2009).

\section{Legitimationsprobleme in der politischen Arena}

Was organisierte Netzwerke von anderen Organisationen der Zivilgesellschaft wie NGOs, Think Tanks u. a. unterscheidet, ist die Tatsache, dass organisierte Netzwerke Konflikt und Unterschied als wichtigste Ressource für Wachstum und Innovation verstehen und weniger der 
Multi-Stakeholder-Logik üblicher Einfluss nehmender Third Party Enforcer (Priddat 2005b) folgen. Als ernstzunehmende Akteure in der politischen Sphäre, gerade in Hinblick auf eine Interessensvertretung, sind organisierte Netzwerke deshalb in der Regel noch nicht legitimiert.

Bisherige, eher auf Repräsentation denn Kooperation ausgelegte Institutionen sind wiederum durch ihre unflexiblen Strukturen jedoch in der Regel oft nicht geeignet, die heterogenen Interessen einer Vielzahl hybrider Akteure zu vertreten und ihre Relevanz zu legitimieren. Eine kreative Lobby, die in der Lage wäre, beispielsweise das komplexe Feld der kreativen Ökonomien zu vertreten, steht bislang aus. Offen bleibt, ob sich eine adäquate Interessensvertretung für die kreativen Ökonomien überhaupt als klar abgrenzbare Organisation zu erkennen geben würde, oder ob die Einflussnahme nicht vielmehr über einen komplexen und sektorenübergreifenden Wechselwirkungsprozess der verschiedenen Akteure und Akteursgruppen erfolgen muss.

Was bedeutet dies jedoch für eine Einflussnahme einer Heerschar vernetzter, kreativer Kleinstunternehmer? Wie sehen die Third Party Enforcer der kreativen Ökonomien aus, und wie die Partizipationsarenen? Einige Initiativen, die sich politisch definieren, erschaffen über die Form des organisierten Netzwerks bereits Alternativen und Gegenentwürfe zu bestehenden, aber ihrem ursprünglichen Zielvorhaben nicht mehr gerecht werdenden Institutionen wie etwa die virtuell organisierte Edu Factory als kritische Antwort auf Universitäten, die immer mehr dem Diktat der freien Wirtschaft unterliegen (www.edu-factory.org), oder EXGAE als Reaktion auf eine nicht mehr zeitgemäße aber nach wie vor politisch forcierte Interessens- und Urheberrechtsvertretung für Kulturschaffende in Spanien (www.exgae.net).

Ziel kann es jedoch nicht sein, verschiedene parallele Systeme zu gestalten und subkulturelle Kompensationslösungen für bestehende Defizite zu suchen. Vielmehr gilt es, den Akteuren der kreativen Ökonomien mehr Einflussnahme und Gestaltungsspielräume zu ermöglichen auch im etablierten politischen Bereich. Die Herausforderung liegt darin, alte und neue Systeme und Logiken miteinander zu verbinden, also eben Schnittstellen zwischen den Systemen entstehen zu lassen und entsprechend zu gestalten. Während die Bedeutung der Schnittstellendimension auf Akteurs- und Wirtschaftsebene bereits verstärkt erkannt wird (werden muss, denn immer mehr Unternehmen sichern sich ihren Wettbewerbsvorteil heute nur noch über die Einbindung externer kollektiver Intelligenz wie etwa über Crowdsourcing) steht dies auf politischer Ebene noch aus. Jedoch zeigt sich am Beispiel verschiedener politischer Programme und Initiativen zur Förderung der kreativen Ökonomien, zuletzt in Deutschland die Initiative Kultur- und Kreativwirtschaft der Bundes- 
regierung, dass das Interesse an dem bislang schwer zu fassenden Feld der Kreativwirtschaft durchaus vorhanden ist.

\section{Neue Intermediäre zwischen Politik und Gesellschaft}

Bei der Frage nach der Steuerbarkeit kreativer Ökonomien wird deutlich, dass es intermediärer Institutionen bedarf, die beide Logiken beherrschen: die koordinierende (repräsentationale) sowie die kooperative (sich selbst organisierende). Die Bedeutung des kulturellen Intermediären an der Schnittstelle zur Politik haben Länder wie Großbritannien, Schweden oder die Niederlande bereits erkannt und teilweise sogar auch institutionalisiert. So hat Großbritannien die staatlich geförderte Institution NESTA (National Endowment for Science, Technology and the Arts) ins Leben gerufen, die als Informationsplattform, Inkubator und Forschungs-, bzw. Beratungseinrichtung fungiert (www.nesta.org.uk).

Schweden hat mit Hilfe der Knowledge Foundation die größtenteils privat finanzierte Vernetzungsinitiative Upplevelse Industrin gestartet, deren Ziel es ist, die schwedischen Experience Industries über den gezielten Ausbau kreativer Hubs in enger Zusammenarbeit mit den Akteuren der kreativen Ökonomien in ganz Schweden zu stärken (www.upplevelseindustrin.se). Onlineportale wie das Amsterdam Creativity Exchange, initiiert durch einen privaten Think Tank unter Einbindung öffentlicher Akteure, bieten einen Vernetzungsservice für kreative Akteure in der Amsterdamer Region, der in der Logik eines Social Networks funktioniert und somit von den Akteuren selbst gestaltet wird (www.acx.nu).

Um den heutigen Anforderungen hochdynamischer Arbeitskulturen gerecht zu werden, bedarf es nicht zuletzt auch auf politischer Ebene, bzw. in den Verwaltungen entsprechende ,intelligent agents“ (siehe Beitrag von Andy Pratt in diesem Buch). Hier ist das Bewusstsein für Schnittstellen hier in der Regel noch unterentwickelt und aufgrund starrer, hierarchischer Strukturen, die der Logik des Industriezeitalters entsprechen, existieren nur wenig Berührungspunkte $\mathrm{zu}$ den informellen Netzwerken der kreativen Akteure. Instrumente wie der strukturierte Dialog oder Branchenhearings, Round Tables, Expertengespräche, Ausschreibungen und Wettbewerbe sind erste Schritte hin zu einem verbesserten, prozessorientierten Dialog mit kreativen Akteuren. 


\section{Fazit}

Die vorangegangene Beschreibung zeigt, dass die Kultur der Schnittstelle ein wesentliches Merkmal der kreativen, wissensbasierten Ökonomien darstellt und als solche strategisch adressiert werden kann und sollte. Die Akteure dieser meist kleinteiligen, stark relationalen Patchworkökonomien entwickeln zunehmend hybride Tätigkeitsprofile, um sich in dynamischen, projektbasierten und netzwerkorientierten Arbeitszusammenhängen flexibel zu verorten und zugleich Differenz herzustellen. Sie bewegen sich in einem ständigen Wechselspiel aus Gefahr (Risiko der Prekarität) und Chance (Autonomiegewinn) und finden individuelle, situationsorientierte Lösungen für den Umgang mit Ungewissheit und Wandel. Die in Netzwerken und Schwärmen organisierten Kleinstunternehmer operieren mit Strategien des kulturellen Hackings oder Vermischungstechniken wie der Bricolage, um sich Handlungsarenen anzueignen und bestehende Ordnungen zu unterwandern. Die globalisierten kreativen Ökonomien sind gekennzeichnet von einer Open Sourceinspirierten Selbststeuerung und haben damit wenig mit den zentralistischen, hierarchischen Steuerungslogiken des Industriezeitalters gemein. Eine hohe Schnittstellenkompetenz gehört zur Grundvoraussetzung, um „mitmischen“ zu können, und damit auch die Fähigkeit zur Improvisation, Kooperation und Reorganisation.

Gerade vor dem Hintergrund gegenwärtiger massiver Transformationen in allen Bereichen der Gesellschaft und dem Infragestellen bisheriger (Un-)Ordnungen wird den kreativen Ökonomien und ihren Akteuren, die alltäglich mit Unsicherheit, Krise und Veränderung umzugehen wissen, eine besondere Vorreiterrolle zuteil. Die derzeitigen globalwirtschaftlichen Entwicklungen wie die internationale Finanzkrise halten für die Bewertung der kreativen Ökonomien einen Erkenntnisgewinn bereit: Es deutet sich an, dass die kleinteiligen, flexiblen, flachen Strukturen der Netzwerkgesellschaft deutlich robuster und damit krisenresistenter sind, als es die monolithischen, starren, hierarchischen Strukturen des Industriezeitalters zu sein vermögen. Und auch Kreativität und soziales Kapital, welche die maßgebliche Ressource der kreativen Ökonomien darstellen, werden nur unwesentlich durch den Zusammenbruch der Finanzmärkte beeinträchtigt, eher noch deuten sich hier sogar hohe Potenzialitäten im Sinne eines nachhaltigen, sozialen Wirtschaftens an. Neue Formen der Vergemeinschaftung, die auf den Werthaltungen kreativer Ökonomien in der Wissensgesellschaft beruhen - Kultur des Teilens und der Teilhabe, global verantwortliches Handeln mit lokaler Verortung, Generierung eines sozialen und kulturellen Mehrwert, Nachhaltigkeit bei gleichzeitiger Transformationsfähigkeit - ordnen das Organi- 
sations- und Institutionengefüge neu und können wichtige Impulse geben. Es wird sich deshalb auch für andere, etablierte Wirtschafts- und Handlungsfelder lohnen, die Phänomene in den kreativen, wissensbasierten Ökonomien genauer in den Blick zu nehmen. Denn darin findet sich eine Vielzahl von Ansätzen für den innovativen Umgang mit Krise und Komplexität.

Schnittstellenakteure, ob nun als Einzelperson oder intermediäre Institution, üben in der Transformation vom Industriekapitalismus zur globalisierten kreativen Wissensökonomie eine wichtige gesellschaftliche Integrationsfunktion aus. Damit die Kompetenz, gerade in Umbruchsituationen Situationspotenziale zu erkennen und optimal auszuschöpfen, nutzbar gemacht wird und Schnittstellenkulturen (mit all ihren neuen Organisationsformen und Nischenmärkten) künftig nicht nur als neue Alternative zu den noch weitestgehend präsenten Strukturen einer industriellen, repräsentationalen Wirtschaftslogik koexistieren, bedarf es einer Professionalisierung der Schnittstellenakteure und ihrer Organisationsformen. Es bedarf neuer Formen strategischer Schnittstellengestaltung. Die Herausforderung besteht darin, Anschlussfähigkeit herzustellen - in unterschiedliche Richtungen: Professionalisierung der Akteure und ihrer (selbst-)organisierten Netzwerke in Richtung Markt und politischer Arenen der Einflussnahme, sowie eine Öffnung der etablierten politischen Institutionen, aber auch staatlicher Ausbildungsstrukturen, in Richtung einer netzwerkorientierten, partizipativen Praxis. Hier bieten intermediäre Institutionen, Vernetzungsplattformen/-initiativen und Inkubatoren, die staatlich und/oder privat unterstützt und gelenkt werden, möglicherweise eine sinnvolle Brückenfunktion. Auch organisierte Netzwerke können zu Strukturen der Teilhabe und Einflussnahme in politischen Entscheidungsprozessen werden. Es gilt, nicht nur neue und alte Institutionen und Haltungen nebeneinander existieren zu lassen, sondern zeitgemäße Formen einer pluralistischen Integration und Schnittstellengestaltung zu finden, um Wachstums- und Innovationspotenziale optimal zu stimulieren.

\section{Literatur}

Blechinger, Gerhard/Milev, Yana (Hg.) (2008): Emergency Design. Designstrategien im Arbeitsfeld der Krise, Wien: Springer.

Boltanski, Luc/Chiapello, Eve (2006): Der neue Geist des Kapitalismus, Konstanz: UVK-Verlagsgesellschaft. 
Bradley, Will/Hannula, Mika/Ricupero, Cristina/Superflex (Hg.) (2006): Self-Organisation. Counter-Economic Strategies, Berlin/New York: Sternberg Press.

Brellochs, Mari/Schrat, Henrik (Hg.) (2005): Raffinierter Überleben. Strategien in Kunst und Wirtschaft. Produkt und Vision, Berlin: Kulturverlag Kadmos.

Borries, Friedrich von (2008): „Emergency Urbanism - Branding als Zukunft der Stadtgestaltung?“ In: Blechinger, Gerhard/Milev, Yana (Hrsg): EmergencyDesign. Designstrategien im Arbeitsfeld der Krise, Wien: Springer, S. 129-136

Bronfen, Elisabeth/Marius, Benjamin/Steffen, Therese (Hg.) (1997): Hybride Kulturen. Beiträge zur anglo-amerikanischen Multikulturalismusdebatte, Tübingen: Stauffenberg Verlag

Bullen, Andrew/Szita, Jane (2007): „Redefining the Guild“. In: Brickwood, Cathy/Ferran, Bronac/Garcia, David/Putnam, Tim (Hg.): (Un)Common Ground - Creative Encounters across Sectors and Disciplines, Amsterdam: Bis Publishers, S. 62-67.

Burt, Ronald S. (2005): Brokerage and Closure: An Introduction to Social Capital, Oxford, New York: Oxford University Press.

Deleuze, Gilles (1992, Orig. 1968): Differenz und Wiederholung, München: Fink.

Friebe, Holm/Lobo, Sascha (2006): Wir nennen es Arbeit oder: Intelligentes Leben jenseits der Festanstellung, München: Heyne.

Friebe, Holm/Ramge, Thomas (2008): Marke Eigenbau: der Aufstand der Massen gegen die Massenproduktion, Frankfurt/Main: Campus Verlag.

Han, Byung-Chul (2005): Hyperkulturalität. Kultur und Globalisierung, Berlin: Merve Verlag.

Hardt, Michael/Negri, Antonio (2004): Multitude. Krieg und Demokratie im Empire, Frankfurt/Main: Campus Verlag.

Hardt, Michael/Negri, Antonio (2003): Empire. Die neue Weltordnung, Frankfurt/Main: Campus Verlag.

Krajewski, Markus (Hrsg) (2004): Projektemacher. Zur Produktion von Wissen in der Vorform des Scheiterns, Berlin: Kulturverlag Kadmos.

Lange, Bastian (2007): Die Räume der Kreativszenen: Culturepreneurs und ihre Orte in Berlin, Bielefeld: transcript Verlag.

Leadbeater, Charles/Oakley, Kate (1999): The Independents. Britain's New Cultural Entrepreneur, London: Demos.

Leadbeater, Charles (2008): WE-THINK: Mass innovation, not mass production: The Power of Mass Creativity, London: Profile Books. 
Lévi-Strauss, Claude (1968, Orig. 1962): Das wilde Denken, Frankfurt/Main: Suhrkamp.

Liebl, Franz/Düllo, Thomas (2005): Cultural Hacking: Die Kunst des Strategischen Handelns, Wien: Springer.

Lotter, Wolf (2009): Die kreative Revolution. Was kommt nach dem Industriekapitalismus? Hamburg: Murmann Verlag.

Osten, Marion von (Hg.) (2003): Norm der Abweichung, Zürich: Edition Voldemeer.

Putnam, Tim (2007): „Creative Spaces and the Knowledge Economy“. In: Brickwood, Cathy/Ferran, Bronac/Garcia, David/Putnam, Tim (Hg.): (Un)Common Ground - Creative Encounters across Sectors and Disciplines, Amsterdam: Bis Publishers, S. 50-56.

Priddat, Birger P. (2005a): Unvollständige Akteure: komplexer werdende Ökonomie, Wiesbaden: VS-Verlag für Sozialwissenschaften.

Priddat, Birger P. (2005b): Strukturierter Individualismus. Institutionen als ökonomische Theorie, Marburg: Metropolis Verlag.

Priddat, Birger P. (2009): Politische Ökonomie. Neue Schnittstellendynamik zwischen Wirtschaft, Gesellschaft und Politik, Wiesbaden: VS-Verlag für Sozialwissenschaften.

Raymond, Eric Steven (2001): The Cathedral \& the Bazaar. Musings on Linux and Open Source by an Accidental Revolutionary, Sebastopol, CA: O'Reilly \& Associates.

Rossiter, Ned (2006): Organized Networks. Media Theory, Creative Labour, New Institutions, Rotterdam: NAi Publishers.

Sennett, Richard (1998): Der flexible Mensch, Berlin: Berlin-Verlag.

Stanitzek, Georg (2004): „Der Projektmacher. Projektionen auf eine ,unmögliche“ moderne Kategorie“. In: Krajewski, Markus (Hg.): Projektemacher. Zur Produktion von Wissen in der Vorform des Scheiterns, Berlin: Kulturverlag Kadmos, S. 29-48.

Tapscott, Don/Williams, Anthony D. (2007, Orig. 2006): Wikinomics. Die Revolution im Netz, München: Hanser.

Virno, Paolo (2005): Grammatik der Multitude. Untersuchungen zu gegenwärtigen Lebensformen, Berlin: ID Verlag.

Wellmann, Inga (2007): „Creativity Makes the World Go Round. Wenn Kreativität zur globalen Währung wird und wir überall Wellenreitern begegnen“. thinktank Nr. 4, Berlin: berlinpolis.

Williamson, Oliver E. (1975) Markets and Hierarchies, New York: Free Press. 


\title{
Hybrid Space Lab (Amsterdam/Berlin)
}

\author{
A WRITTEN INTERVIEW WITH ELIZABETH SIKIARIDI AND \\ FRANS VOGELAAR - QUESTIONS BY INGA WELLMANN
}

\section{What is Hybrid Space Lab?}

Frans Vogelaar (FV): Hybrid Space Lab is an interdisciplinary environment characterized by an innovative and integrated approach to spatial issues. It specializes in strategic research and high-end design. It is an $R \& D$ and design practice that focuses on the hybrid fields that are emerging through the combination and fusion of environments, objects and services in the information/communication age. Hybrid stands for our strategy of combining fields, Space reflects our expertise and Lab stands for our innovative and experimental approach. Our web address ends in .net, suggesting our working practice of involving a network of specialists.

\section{What concept does the name reflect?}

Elizabeth Sikiaridi (ES): Mixing fields, combining expertises and considering environments in their multiplicity of dimensions is for us a method for finding new relevant solutions to increasingly complex spatial design challenges. We investigate actual developments in the general fields of culture, communication, production, exchange (markets) as well as technological innovations, not to foremost monitor market development but in order to generate visions.

$F V$ : The starting point and spearhead of our work lie in the fusing of digital and analog environments, in embedding media networks in urban, architectural, social and cultural space. Our environments are today being rapidly transformed by medialization - and hence globalization. Today's technological developments are accelerated by the fusions of 
information technology, nanotechnology, biotechnology and neurotechnologies (brain technologies) and their convergence into new hybrid technology platforms such as DNA computing, nanobiotechnology, synthetic biology and neuroengineering... We approach these technological developments from the perspective of the designer, the architect and the urbanist, by 'inhabiting technology', by transforming these technological developments to meet the way we want to live.

$E S$ : Innovation is not a goal in itself, but a means of coping with and steering developments. Hybridization is an evolutionary strategy to develop new solutions for the changing environments in our fast-moving world. I remember an early reading of an essay entitled Race and History that Claude Lévi-Strauss wrote for UNESCO in 1952. Lévi-Strauss contrasts cumulative, dynamic, moving cultures with static, nondeveloping cultures and argues that intercultural connections and crossfertilization are crucial to the development of differentiated civilizations.

\section{What kind of projects do you work on and initiate?}

$F V$ : Hybrid Space Lab is a lab and a network in which designers, architects, urbanists, landscape architects, environmental planners, software and hardware engineers, and media artists collaborate to develop projects for combined analog and digital, urban, architectural, design and media spaces. The relationship of digital service environments and artifacts to our architectural and urban surroundings and objects is central to our strategic research and design agenda. The research and development projects range from urban games and urban planning to buildings, architectural interiors and industrial design applications and wearables.

ES: We also have a long track record in consultancy; we were part of a think tank for the Dutch government and have advised corporations, local authorities, and cultural, educational and research institutes. We developed the project idea for the Urban Dinners on the main highway of the Ruhr region for the RUHR.2010 Cultural Capital organization. Our working method is to merge analytical investigations with synthetic design processes. We apply Design Thinking to a very extensive range of issues and fields.

$F V$ : We work internationally. At the moment we are busy with a project for the Tschumi Pavilion Foundation in Groningen in the Netherlands, consisting of a programmatic plan, a business plan, an urban strategy and an architectural design for a new Media and Performing Arts Centre. We are also in the final stages of a project for the Torino World Design 
Capital 2008, geared to security in public space - politically, a heavily abused issue in Italy. Our approach is based on the appropriation of public space by proposing and designing a series of services that strengthen and encourage public involvement in the open spaces of the city. Last but not least, we are curating an international touring exhibition on cityscape and sustainability and designing a 'Media Camp' due to be built in Shenzhen by the end of 2009 .

\section{Where does the Hybrid Space Lab come from (i.e. your background story/out of what need did you set the ground for the HSL)?}

ES: Frans studied industrial design and architecture and urbanism; I studied architecture and urbanism. Before setting up our practice we had already gained professional experience in many different fields, including architecture (OMA/Rem Koolhaas, Behnisch \& Pa.); industrial design (Studio Alchemia/Alessandro Mendini); and fashion (trend research in Paris), as well as urbanism; landscape architecture and urbanism; material design, food design and exhibition design; interior architecture; media design... We both have hybrid cultural backgrounds and have lived and worked internationally: I was born in London, grew up in Athens, studied at the École d'Architecture de Belleville in Paris and at the Technical University of Darmstadt. Frans was born in the Netherlands, grew up in Zimbabwe, studied at the Design Academy in Eindhoven and at the Architectural Association School of Architecture (AA) in London.

$F V$ : We were aware from early on that the ICT developments, the rise of a network society as Manuel Castells puts it, would have an immense impact on the way we live and work, accelerating globalization, the flexibilization of lifestyles and the development of the creative economy. The new production and communication tool of the networked computer provides a common working instrument for a broad range of creative professions. It is also transforming creative processes and products and paving the way for a series of hybrid professional fields.

ES: At first, when people were thinking in terms of the polarity of media and physical space, it was difficult to communicate our 'hybrid space' approach, which considers the physical environment in the context of and in relation to the networks that it belongs to and interacts with. We started with self-initiated artistic research projects. These led to consultancies and research and development and design assignments. 
Where do you locate yourself within the broader context of the creative economy? Would you consider yourself to be an intermediary institution at the interface of several disciplines?

ES: The creative economy is, by definition, dynamic. The traditional disciplines will develop, transform, mutate, fuse. We see our 'undisciplined' laboratory as a catalyst in this process and as a space for development and experimentation.

What are your strategies in overcoming outdated structures or practices?

$F V$ : When we started, we did have a communication problem as we did not fit neatly into the predefined territory of the distinct disciplines. We were repeatedly asked: What exactly are you? Designers, architects, urbanists, landscape urbanists, researchers... or media artists? We responded with an offensive strategy, by defining our office as a hybrid laboratory.

What definition or interpretation of the 'hybrid' do you feel is of relevance for contemporary phenomena in the economical, social and cultural sphere? Where and how does the phenomenon of the hybrid transform the way we work, share and use space, organize ourselves and others, develop new institutions, identities and job profiles, and so forth?

$E S$ : In the times of the Aristotelian categories, the notion of the 'hybrid', the crossbreed, had a negative connotation. Today the notion of the 'hybrid' is everywhere. Hybridization is becoming an increasingly important issue in the cultural field. Look at the attention paid to world literature; think of the 2008 Nobel Price for Literature. Today, you have hybrid cars, hybrid businesses, hybrid securities, hybrid plastics, hybrid plants, hybrid pigs... all reflecting a cultural shift away from a mindset based on clear-cut categories towards a flexible approach based on intermixtures, on interconnections and networks.

$F V$ : Hybrid space is everywhere in our daily lives. Take, for example, the private (communication) space of mobile telephony, which creates islands of private space within public urban space. Or monitored environments, where cameras keep watch over open urban areas. The teleworkplace is becoming an integral part of the home, the office is becoming a space for encounters, with a meeting lounge atmosphere, the (connected) car is becoming a mobile extension of our networked existence, to name only a few examples. 
ES: Hybrid defined as the combination - the fusion - of the analog and the digital is a good starting point. As Frans just mentioned, medialization and globalization are transforming space, activities and professional fields. Hybrid retail, integrating e-commerce and physical stores, changes the shop into the experiential space of the point of sale. Hybrid publishing is closely entwined with citizen journalism. In new business models customers are co-creating and co-developing products. Think of the Lego Digital Designer that enables users to create, share and improve the design of the building brick toys.

$F V$ : The days when people were defined by their profession and remained with the same employer and in the same professional field throughout their working life belong in the past. Together with my students at the Academy of Media Cologne we are developing strategies for 'hybrid practice'. The aim is to generate new practice models for the multi-job strategies and the patchwork biographies of the 'project workers' of today and tomorrow.

\section{In the past, who and what were the forerunners and idols of today's hybrid practice?}

ES: One of the pioneers was the Greek and French music composer, media artist, architect and engineer Iannis Xenakis (1922-2001). In the 1990's we worked with Xenakis in his personal archives in Paris and interviewed him on his work and artistic concepts. What is interesting about Xenakis is the way he implemented structures in different fields and transferred them from one field to another: from engineering and mathematical-scientific research to music, from music to architecture and to visual events. Xenakis worked on the poetics of the electronic age, as he called it, integrating the new, universal, electronic tools of the computer in his artistic oeuvre. He argued for the development of a general discipline of form, a general morphology, an interdisciplinary effort, corresponding with his universal thinking and his practice of 'transfer'.

FV: Another pioneer is the American designer, architect, author and visionary inventor, Richard Buckminster Fuller (1895-1983), who was deeply concerned with sustainability issues. 
And, with regard to the book title, what do you consider important for the governance of the creative economy? Is there a special need for new intermediaries, both on an individual and an institutional level? Will it be important to find new modes of representation for the 'in-betweens' - such as a lobby for creative intermediaries, or new institutional interfaces?

$E S$ : It is important to reflect on and discuss these themes. We still need to develop not only working business models for the creative sector but also social strategies to support the sustainable development of a creative society and economy.

$F V$ : We believe that it is important to strengthen the interdisciplinary networking platforms and support creative environments of encounter because they foster innovation. One shouldn't underestimate the strategic value of cross-over spaces and hybrid laboratories.

Abbildung 8: 'wir ESSEN FÜR DAS RUHRGEBIET' or the urban dinners on the 'Ruhrschnellweg A40/B1'.

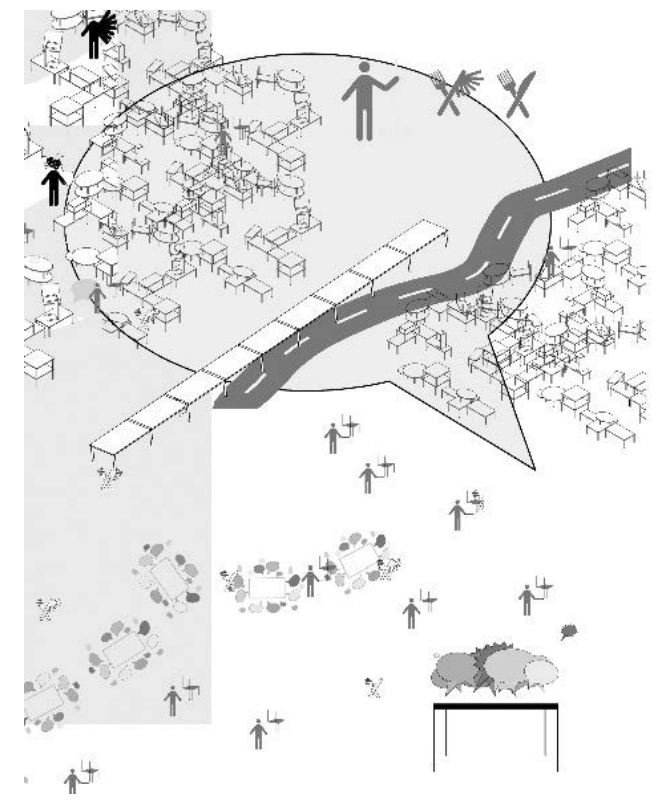

Quelle: Hybrid Space Lab, Amsterdam/Berlin. 
IMAGINEERING 



\title{
Narrating Urban Entrepreneurship: A Matter of Imagineering?
}

\author{
CHRIS STEYAERT, TIMON BEYES
}

A city that does not curate its image and manage its story is out of date.

Sharon Zukin

Then, there are the myriad experiments that set out to invent flexible models of imagination and narrative outside the enforced routines of consumption.

Nigel Thrift

The battle between cities with regard to their creative possibilities has evolved into a process of multiplying ever-new images and variegated stories of urban attractiveness and success. Engineering 'cool' images and 'hot' stories about one's city is now a central endeavor in the narratives of urban policy-making that center more and more on the idea of the entrepreneurial city. The making of an entrepreneurial image is enacted through various narrative genres that lie somewhere between place making and place marketing, between branding and boosting, between restoration and revanchism, between iconic architecture and megaspectacle. This 'imagineering' is not only part of the way cities try to (re-)present themselves as entrepreneurial to various audiences through a real 'image inflation' (Zukin 2008: xii) but is also inscribed in the various ways urban creativity and entrepreneurship can be studied, researched and imagined.

In this chapter we aim to differentiate the political narratives of the entrepreneurial city as we emphasize the need to understand the politics 
of narration and make a plea for critical reflexivity in our forms of researching and theorizing. We will thus try to investigate how the politics of narration is intertwined with the narration of political concepts and will argue that the narrating of urban entrepreneurship can raise very different images and discourses of city life beyond those that are currently engineered. We will distinguish between a grand narrative, a counternarrative, and an assemblage of more ambivalent little narratives, which we call prosaic narration. While the distinction between these three types might be seen as a bit too simple and 'straight', we believe that by juxtaposing these different forms of narration and alternating between them, we can help problematize the engineering of the city as entrepreneurial and imagine alternative views both of city life and of what is understood as its creativity.

Reflexivity requires that we reflect carefully upon the ways of examining how the relationship between cities, entrepreneurship and culture has been established in narratives of policy-making in the last 25 years. Thus, the imagineering of the city is connected to the way that research itself is critical of how certain images and narratives are kept prominent; it can provide other kinds of stories in which the city is addressed as another kind of space, a heterotopia (Foucault 1986; Steyaert 2006). We will argue that it is time to go beyond the choice between a celebratory and a critical analysis of urban entrepreneurialism. By investigating cities through non-representational narratives, we can orient the narration of urban entrepreneurialism towards a politics of everyday life (Thrift 2008), 'a politics of what happens' (Thrift 2008: 2) and of the 'ordinary' city (Robinson 2006).

\section{The rise of the entrepreneurial city and the grand narrative of the creative class}

Whether we look at Manchester which 'has long been seen as the definitive entrepreneurial city' (Mace/Hall/Gallent 2007: 60; see also: Quilley 2000; Williams 2003) or Barcelona which convincingly connects the spectacle of new and old architecture with economic dynamism (McNeill 2001; Marshall 2004; Luna-Garcia 2008), an almost endless series of cities have been called 'entrepreneurial' or have been given a 'creative label'. The list includes former industrial towns and postmodern cities, middle-sized towns and world cities. No end of how - to books provide readers with tools to develop their cities as entrepreneurial (Goldsmith 1999) or creative (Landry 2000). All of this activity il- 
lustrates how it has become bon ton to combine city development with entrepreneurship, creativity and culture.

The turn to the entrepreneurial city can be seen as part of a wider 'entrepreneurial shift' since the 1980s (Locke/Schöne 2004; Steyaert 2007) which also suggests that we connect the trope of the entrepreneurial city with the creativity discourse. The latter's emergence at the dawn of the entrepreneurial shift is illustrated by a book by Åke Andersson (1985), a Swedish professor of regional economy. In Kreativitet: Storstadens Framtid he outlines the role of creativity for future urban development, taking Stockholm as its case study. This book can be said to be a pioneering work, anticipating the next waves of creativity, carried out by Landry (2000) and Florida (2002), among others.

As the book was never translated, it was not likely even to make it into a footnote, but Peter Hall picked it up in his urban epos on Cities in Civilization: 'So the Swedes are right: creative cities, creative urban milieux, are places of great social and intellectual turbulence, not comfortable places at all' (1999: 285-286). Hall's 'magisterial book' (LeGates 2000: 201) presents an expansive overview of cases and theories to explain how cities have evolved into 'golden ages' or 'belles époques', forming creative crucibles and innovative milieus. Emphasizing social and cultural turmoil, Hall argues that "creative urban societies often emerge as new classes, whose wealth derives from entrepreneurship and trade, and who challenge traditional propertied elites' (ibid.: 201). Hall connects the emergence of creativity with cosmopolitanism based on an influx of young immigrants and proposes that a fluid class structure, and the tension this brings along between old and new social groups, fosters innovation. Thus his position is that creativity is part of a tension between 'classes' rather than involving the performance of a 'new, creative class'.

The latter view became prominent at the time Hall's book was published. During the wave of the so-called 'new economy' the connection between cities, creativity and economic success became a dominant formula (Hjorth/Steyaert 2003). While the belief in the new economy faded quickly, Richard Florida (once an urban planner, now a professor of business and creativity in Toronto), was able to elevate this 'new credo of creativity' (Peck 2005: 740) to a wide acceptance in circles of (urban) policy makers as a kind of 'new new economy' (ibid.: 743). Florida argues that urban economic development is to be formed within a cocktail that includes entrepreneurship, creative life styles and a diverse, creative class. Florida's logic of argumentation - which Peck (ibid.: 741) calls 'a sales pitch' - aims to re-install a grand narrative 
(Lyotard 1984), which promotes an optimistic, if not utopian image of urban policy making, and is supported by a practice of boosterism.

What Florida (2002) sees as the 'rising' creative class is a group of so-called creative 'professionals' - from artists to scientists, from entrepreneurs to venture capitalists - who turn their lifestyles, values and tastes, as well as their relationships, into the main point of departure for combining work, leisure and living; in doing so they seem to complement such identifiable 'classes' as the working, service and agriculture classes. Creativity thus gravitates to specific locations, as creative people tend to 'cluster in places that are centers of creativity and also where they like to live' (Florida 2002: 7). For Florida, this is not a small change, but a 'sea-change'; indeed, 'it is the emergence of a new society and a new culture $-[\ldots]$ a whole new way of life' (ibid.: 12).

In addition to attracting talented professionals, Florida further advises cities to seek technological prominence and to encourage a multicultural environment. These three elements are combined in a magic formula of 3 Ts: talent is connected with technology and tolerance. In this formula, the connection to art and culture is not first on the list, but art is seen as the close associate that combines well with technological nerds and with cosmopolitan and queer lifestyles. In an interview as he launched his book in 2002, Florida summarized his view by stating that 'cities must attract the new 'creative class' with hip neighborhoods, an arts scene and a gay-friendly atmosphere - or they'll go the way of Detroit' (Dreher 2002: 1; quoted in Peck 2005: 740).

As a consequence, Florida inscribes a strange mixture of figures into a narrative of mega-optimism and elitism. Artists, nerds, homosexuals and others are needed to enact the urban imagineering projects and to play a prime role in aestheticizing the urban landscape and concocting spectacles and mega-events. Art, sexuality, and in the end, city life itself, become commodities. For instance, Florida's emphasis on the gay and lesbian community has been contested both by conservatives who find that it undermines the values of family life so central in these conservative narratives (Peck 2005) and by the gay and lesbian community which finds itself staged in a spectacle of creativity where 'queer difference is now exploited as a material and semiotic resource in the commodification of the city' (Grundy 2003: 4). 


\section{A counter-narrative: Harvey's concept of urban entrepreneurialism}

Those who currently embrace the politics of the entrepreneurial city by subscribing to the grand narrative sketched out above must remember that this idea is part of a lasting, entrepreneurial shift which was masterfully captured by David Harvey (1989). In his seminal article in the Geografiska Annaler, he describes how the discourse of urban governance moves from managerialism to entrepreneurialism. While the discourse of the entrepreneurial city might try to present itself as 'new', Harvey situates the rise of the connection between the city and entrepreneurship in the 1970s: 'the shift from urban managerialism to some kind of entrepreneurialism remains a persistent and recurrent theme in the period since the early 1970s' (1989a: 5). Thus, according to Harvey, the phenomenon of civic boosterism and entrepreneurialism has long been a major feature of urban systems. It arose when the economic and fiscal base of many large cities started to erode, especially in industrial cities; then, it was argued, cities required new and innovative models of governance.

The discourse about a 'spatial economy' (Fujita/Krugman/Venables 2001) began at economic colloquia as advocates of a closer link between the public and private sectors also held government responsible for promoting local areas to attract new businesses and entrepreneurial activity. The new adage of governance was 'to maximize the attractiveness of the local side as a lure for capitalist development' (Harvey 1989a: 5). Goodman's (1979) assessment of government as 'the last entrepreneurs' illustrates the belief in the urgent application of the entrepreneurial recipe, even if it did not mean the end of this argument (du Gay 2004).

Thus Harvey's analysis is timely as it connects to the developments towards what has been described as a post-industrial, post-Fordist, postmodern metropolis (Soja 2000), where new modes of organizing transport, work and shopping change the appearance of cities, along with their social structure, which can be characterized as 'a veritable archipelago of elite enclaves, fragmented neighbourhoods and "edge" cities' (Hubbard/Hall 1998: 1). This was the era of the enterprise culture as instigated by the neo-liberal economic politics of Thatcher and Reagan, which propelled the entrepreneur to the forefront as the symbolic figure of the new imaginary of urban (economic) life. This was the era of the yuppie culture where the golden boys of Wall Street were keen to sublimate their profits in narcissistic lifestyles (Hjorth/Steyaert 2006).

However, this entrepreneurial optimism, that brings forth 'a futuristic vision of a visually enticing city of dreams', is 'entwined with a post- 
apocalyptic scenario of urban unrest, deprivation and despair' (Hubbard/Hall 1998: 1). Such dystopian images notwithstanding, what Harvey finds most striking is the 'general consensus' then 'emerging throughout the advanced capitalist world that positive benefits are to be had by cities taking an entrepreneurial stance to economic development' (1989a: 4). In fact, he sees it as remarkable 'that this consensus seems to hold across national boundaries and even across political parties and ideologies' (ibid.: 4).

Harvey (1989a: 8) focuses on three features of this entrepreneurial shift. First, as the influence of business interests increases, so does the number of public-private partnerships. Second, local governments engage in entrepreneurial and speculative risk-taking and assume activities which had been associated solely with the private sector. Third, the focus shifts from a political economy of territory to one of place where attention is drawn to the construction of a specific place and away from the broader problems of a region or territory. In summary: 'The new urban entrepreneurialism typically rests [...] on a public-private partnership focusing on investment and economic development with the speculative construction of place rather than amelioration of conditions within a particular territory as its immediate (though by no means exclusive) political and economic goal'.

Urban governance in an entrepreneurial mode is then enacted through a combination of strategies: 1 ) respond to international competition based on local advantage and investment; 2) develop a local service-oriented economy; 3 ) assemble a wide range of supportive services in high finance, media and government; and 4) make central resources available regionally. With regard to urban regeneration, Harvey underlines what he calls 'the up-grading of the image' (1989a: 7) of cities and the emphasis on appearance, style, spectacle, display and imagery. 'Above all', he writes, 'the city has to appear as an innovative, exciting, creative, and safe place to live or to visit, to play and consume in' (ibid.: 9, our emphasis). This entails an orientation to quality of life, cultural innovation, postmodern design, consumer attractions (such as convention and shopping centres, marinas, exotic eating places) as well as urban spectacles including festivals and cultural events. For Harvey, it follows that other cities imitate these strategies; then, instead of being unique, cities seem to look more and more the same: 'How many successful convention centres, sports stadia, Disney-worlds, harbour places and spectacular shopping malls can there be?' (1989a: 12). Furthermore, he says the social costs of urban entrepreneurialism are immense; witness the increasing disparity in wealth and income and the processes of 
urban impoverishment, dispossession and displacement (see also Harvey 2008).

In hindsight, Harvey's analysis was most clairvoyant, and in our view is still quite valuable in a contemporary context where cities are increasingly competing on a global scale, selling themselves based 'upon the creation of an attractive urban imagery' (1989a: 13) and being compared and measured with regard to their potential to attract a creative class. When we call his analysis timely, we must remark that Harvey might even have underestimated the increasingly prominent role of culture, creativity and the aesthetic in the shift to urban entrepreneurialism. To be fair, Harvey was already relating the entrepreneurial urbanism to a political aesthetic:

Local coalitions (of city management and the regional private sphere) have no option, given the coercive laws of competition, except to keep ahead of the game thus engendering leap-frogging innovations in life styles, cultural forms, products and service mixes, even institutional and political forms if they are to survive. The result is a stimulating if often destructive maelstrom of urbanbased cultural, political, production and consumption innovations. It is at this point that we can identify an albeit subterranean but nonetheless vital connection between the rise of urban entrepreneurialism and the postmodern penchant for design of urban fragments rather than comprehensive urban planning, for ephemerality and eclecticism of fashion and style rather than the search for enduring values, for quotation and fiction rather than invention and function, and, finally, for medium over message and image over substance (1989a: 12-13).

While this eloquently formulated observation reflects Harvey's analysis of the condition of postmodernity (see Harvey 1989b), twenty years later we see that his critical analysis has not been able to call a halt to the by now overwhelming spread of this urban aesthetic; the situation now requires a complementary, analytical strategy, as we will discuss in the next section.

\section{In search of prosaic narratives}

While the discourse of creativity, occurring in several waves, has increasingly been appropriated into optimistic tales of urban entrepreneurship, it is clear from Harvey's critique that critical voices began quite early to question this success story and utopian-like narrative; they also documented the dystopian side of the connection between city and creativity. Others picked up on Harvey's critique, which was extended, both 
empirically and conceptually, almost ten years later in Hall/Hubbard's (1998) edited volume The Entrepreneurial City, illustrating what they called the 'burgeoning cross-disciplinary literature on urban entrepreneurialism' (ibid.: 3). The book tried to capture the central debates around the entrepreneurial city and to map the new modes of governance implicated in the economic, social and cultural transformation of cities. It provides several illustrations of how changing the image of a locality is a central component of entrepreneurial governance, suggesting that it is perhaps best to consider the entrepreneurial city as an imaginary city, constituted through a plethora of images and representations' (ibid.: 7).

These counter-narratives have been valuable, responding to the tendency to reduce city governance to myth making and spectacle styling, but they have not been able to shift policy-makers from seeing the entrepreneurial city as a preferred script. While we can trace a clear genealogical history from the contemporary narrative of urban creativity back to the entrepreneurial efforts of de-industrialized cities, we must remember that ' $[\mathrm{t}]$ he script of urban creativity reworks and augments the old methods and arguments of urban entrepreneurialism in politically seductive ways' (Peck 2005: 766). The emergence of the creative industries and the valorization of the cultural economy in the 1990s have ossified the association between the urban, the entrepreneurial and the cultural, making them harder to critique. According to Boltanski/Chiapello (2006), among others, the entrepreneurial spirit has recuperated the strategies of artistic critique at the advantage of its own ideology; in doing so, it has made traditional critique look ineffective.

Thus we see a constant attempt to present the relationship between the urban and the entrepreneurial as self-evident, forcing researchers themselves to keep trying to change and recreate their strategies of analysis and critique. We argue that in order to make the relationship between entrepreneurship and the city more ambivalent we need to invent alternative forms of critique that can not only document how the styles of seduction have altered but also affirm other uses of the city-space and invest(igate) in the de-commodification of the city. The question now is how to move beyond the dichotomous representation that divides the analysis of city life in two too clear camps; this analysis seems to (re)present the city as either Pleasantville or Panicville (Virilio 2005), as either urban dream or metropolitan nightmare (Gundle quoted in Harvey 1989a) or as the site of either an 'experience economy' of play and passion (Hjorth/Kostera 2007) or a 'fear economy' of surveillance and security (Davis 2002; Thrift 2005).

This attempt to change the dualistic representation can be illustrated by a minor but, we think, symptomatic recent debate within the field of 
urban studies (Latham 2006a, 2006b; Cochrane 2006). In the Journal of European Urban and Regional Studies, the geographer Alan Latham staged an intervention into what he perceives as the 'limitations' of Anglophone urban studies (2006a: 88); his example is the interest in and the corresponding studies of the (development of the) city of Berlin. Latham identifies a consensus at work in current discussions in Englishlanguage urban studies: an implicitly shared understanding of what is 'driving' the development of the European city, of what processes call for scholarly attention and, therefore, of what is seen and what is left out.

This consensus concurs broadly with the counter-narrative of the entrepreneurial city as geared towards an intensified entrepreneurialism. Consequently, this scholarly consensus extends to the worrisome effects of the 'entrepreneurialization' of cities that are usually denoted by phenomena such as gentrification, boosterism, and gated communities as well as social technologies such as new surveillance mechanisms and Business Improvement Districts. Latham (2003; 2006a) traces several widely shared and interrelated propositions: an intensified orientation towards consumption, a 'hyper-aestheticisation of the everyday' (Latham 2003: 1701) and neo-liberal governmental strategies go hand-inhand with a diagnosed globalization of cities and an increasing polarization by wealth and income as well as increased social exclusion.

The counter-narrative has itself become a compelling and dominant narrative of Anglophone urban literature that, according to Latham, has been 'applied' to Berlin. Apart from studying how Berlin has been reimagined through place-marketing 'and the symbolic spectacle of global architecture' (Cochrane/Jonas 1999: 152, and ignoring for now the fact that these authors also identify the coeval imagineerings of Berlin as national capital and 'normal' city), he sees the new 'invention' of the Potsdamer Platz as the most obvious case that demonstrates the dominance of economic power and consumerist urban development (e.g. Marcuse 1998; Allen 2006). Certainly, the construction of the Potsdamer Platz seems to be an almost ideal-typical example of the 'potential tragedy [...] that the menu from which big cities seem to be permitted to choose their futures appears to remain so limited' (Cochrane/Jones 1999: 161).

The question, therefore, is not whether such analyses might miss the point; in fact, they vividly reveal the power of urban entrepreneurialism at work, so to speak. Rather, the question is what the shared agenda of Anglophone urban studies does not permit us to see. That is, what happens if, in Latham's words, the 'Anglophone consensus [...] comes to be the account that matters most' (2006a: 91)? One risk is that this particular discourse - its significant merits notwithstanding - may fail to notice 
the diversity between (European) cities. As Latham points out, Berlin has a distinct history of urban planning and renewal that, even now, continues to deviate from the clear-cut neo-liberal or 'entrepreneurial' model which apparently determines urban development in larger (Western) cities.

For us a more important danger is in overlooking the 'contemporaneous heterogeneities of space' (Massey 2005: 5): the plurality of spatial trajectories that produce urban spaces. This is not limited to the example of Berlin, of course. But it is somewhat ironic that at the same period when the Potsdamer Platz was being conceived, built and put to use, Berlin was becoming an object of inquiry as a hotbed for experimental, 'autonomous' and often minor spaces, for so-called 'counter-urbanities' in multiple expressions (e.g. Latham 1999; Oswald 2000; Cupers/Miessen 2002; Groth/Corijn 2005), including a host of endeavours to resist or playfully parody consumer culture and the privatization of space. Thus, as Latham puts it, the problem is that through dominant critical patterns of thinking, "we end up with accounts of Berlin which [...] miss many of the more interesting and exceptional phenomena which are shaping Berlin' (2006b: 377).

Both Latham's diagnostic reading of urban theory's dominant (counter-)narrative and his call to open up to the plurality of spatiourban trajectories fit well with our broad distinction between grand narrative, counter-narrating and what we call the prosaic narration of spatial performances that moves beyond orthodox theoretical orderings. Perhaps not surprisingly, artistic performances and their potential to reconfigure what we can perceive, see and speak are of considerable interest here (Rancière 2004; e.g. Beyes 2009). Instead of reinforcing the domestication of artistic events, Amin/Thrift (2002) point out, 'the most exacting, exciting and enticing attempts to produce [...] new modes of belonging have been taking place in contemporary architecture and performance art as they have tried to redefine - in practice - what is meant by place as living rather than lived space' (ibid.: 48, our emphasis).

These kinds of artistic urban interventions are processual, dynamic, not static, and 'they rest on a particular understanding of architecture, somewhat in line with Benjamin's notions of architecture as "tactile appropriation", as constantly being transformed by its use, its boundaries renegotiated by habits.' (ibid.: 49). And yet, the metaphor of performance leads us beyond the realm of art, into very practical imaginations and creations of encounters, affects, unforeseen relations, play, liminality, protest and transformation (Thrift 2000; Thrift/Dewsbury 2000). From serious or carnivalesque performances of resistance (Lyle 2008) to the affective enactments and reorderings of urban geographies by home- 
less people (Cloke/May/Johnsen 2008) or to the reclaiming of the urban agenda by informal actors reanimating indeterminate spaces (Groth/ Corijn 2005), the urban fabric produces manifold manifestations and and new forms of expression which allow change to happen.

Again, this is not to gloss over the oppressive and damaging consequences of entrepreneurial urbanism which the critical counter-narrative lays bare. But it seems all the more urgent to enrich our understanding of cities in neo-liberal times by exploring stories that present alternatives to the dominant critique of urban entrepreneurialism, because it is here that we might 'imagine possible futures beyond the narrow confines of a globalized, neo-liberal, free-market model' (Latham 2006a: 91). Conceptualizing urban space as an effect of assemblages of heterogeneous interrelations and interactions, as an open, unfinished and relational 'becoming space', first and foremost engenders the possibility of politics (Massey 2005: 149ff.). In this sense, the discourse of prosaic narration leaves no other choice than to refrain from advocating for or prescribing a new mode of urban governance. Following Lefebvre's notion of 'the right to the city' (1996) and Amin/Thrift's articulation of a 'politics of the common' (2002), we believe the focus must shift from a particular form of urban democracy and governance to 'the city as a site of politics in motion' (Amin/Thrift 2002: 155): 'The ideal city [...] would be the ephemeral city, the perpetual oeuvre of the inhabitants, themselves mobile and mobilized for and by this oeuvre. [...] The right to the oeuvre, to participation and appropriation (clearly distinct from the right to property), are implied in the right to the city' (Lefebvre 1996: 173ff.; original emphasis).

Inquiries into the mundane and artistic performances of city life cannot be disentangled from the politics of narration and critique. To attend to these prosaic events, academics will need to change their narrative performances by engaging with what Thrift (2008) calls nonrepresentational theorizing. Challenging classic narrations and semiotic accounts and their interest in how meaning is produced and articulated, non-representational accounts 'emphasize bodily and technological engagements with urban space through affect and ontology' (Hetherington/Cronin 2008: 6). Non-representational theorizing can lead to an important shift in understanding how the narration of urban creativity moves from a discursive level of meaning-making to a performative, neo-materialist level that takes on the intensities and affects through which creative space is assembled. Such a different style of narrating can be related to a detailed, prosaic narration (Steyaert 2004) and a performative narration (Thrift 2000), both of which pull in everyday but intense stories and other fragments of urban life, as well as to a narration 
that practices the possibility of fabulation, that is imagining the future becoming of a collective urbanity (Hjorth/Steyaert 2006). Rather than set out a grand political scheme or try to counter it, we suggest that by increasingly circulating little narratives, we can collectively move the engineering of images towards a practice of imagination that can help bring forward an intensive urban life. Beyond a theoretical diagnostics of grand (counter-)narrations, we suggest engaging with a form of living space inquiry by attending to the intensities, connections and blockages in everyday urban interaction. Such an affirmative politics narrates the city as a heterotopia where affects, ideas and possibilities are assembled and where the grand narratives are not denied but are instead deterritorialized and launched again. A heterotopic politics of urban creativity thus requires a different narration of entrepreneurship tout court.

\section{References}

Allen, John (2006): ‘Ambient Power: Berlin's Potsdamer Platz and the Seductive Logic of Public Spaces'. In: Urban Studies 43(2), pp. 441-455.

Amin, Ash/Thrift, Nigel (2002): Cities: Reimagining the Urban, Cambridge: Polity Press.

Andersson, Åke E. (1985): Kreativitet: Storstadens framtid, Stockholm: Prisma.

Beyes, Timon (2009): 'Spaces of Intensity: Urban Entrepreneurship as Redistribution of the Sensible'. In: Hjorth, Daniel/Steyaert, Chris (eds.): The Politics and Aesthetics of Entrepreneurship. Cheltenham: Edward Elgar, pp. 92-112.

Boltanski, Luc/Chiapello, Eve (2006): The New Spirit of Capitalism, London: Verso.

Cloke, Paul/May, Jon/Johnsen, Sarah (2008): 'Performativity and Affect in the Homeless City'. In: Environment and Planning D: Society and Space, 26(2), pp. 241-263.

Cochrane, Allan (2006): 'Euro-commentary: (Anglo)phoning Home from Berlin: A Response to Alan Latham'. In: European Urban and Regional Studies 13(4), pp. 371-376.

Cochrane, Allan/Jonas, Andrew (1999): 'Reimagining Berlin: World City, National Capital or Ordinary Place?' In: European Urban and Regional Studies, 6(2), pp. 145-164.

Cupers, Kenny/Miessen, Markus (2002): Spaces of Uncertainty, Wuppertal: Müller + Busmann. 
Davis, Mike (2002): Dead Cities and Other Tales, New York: New Press.

Du Gay, Paul (2004): "Against "Enterprise" (but not against "enterprise”, for that would make no sense)'. In: Organization 11 (1), pp. 37-57.

Florida, Richard (2002): The Rise of the Creative Class, Cambridge, MA: Basic Books.

Foucault, Michel (1986): 'Of Other Spaces'. In: Diacritics 16 (1), pp. 22-27.

Fujita, Masahisa/Krugman, Paul/Venables, Anthony J. (2001): The Spatial Economy: Cities, Regions, and International Trade, Cambridge, MA: MIT Press.

Goldsmith, Stephen (1999): The Entrepreneurial City: A How-To Handbook for Urban Innovators, New York: Manhattan Institute.

Goodman, Robert (1979): The Last Entrepreneurs: America's Regional Wars for Jobs and Dollars, New York: Simon and Schuster.

Groth, Jacqueline/Corijn, Eric (2005): 'Reclaiming Urbanity: Indeterminate Spaces, Informal Actors and Urban Agenda Setting'. In: Urban Studies 42 (3), pp. 503-526.

Grundy, John (2003): Staging Queer Differences in the Entrepreneurial City: The Politics of Pride Toronto. Doctoral Dissertation: Carleton University.

Hall, Peter (1999): Cities in Civilization: Culture, Innovation, and Urban Order, London: Phoenix Giant.

Harvey, David (1989b): The Condition of Postmodernity, Oxford, UK and Cambridge, MA: Basil Blackwell.

Harvey, David (1989a): 'From Managerialism to Entrepreneurialism: The Transformation in Urban Governance in Late Capitalism'. In: Geografiska Annaler B, 71 (1), pp. 3-17.

Harvey, David (2008): 'The Right to the City'. In: New Left Review 53, pp. 23-40.

Hjorth, Daniel/Kostera, Monika (eds.) (2007): Entrepreneurship and the Experience Economy, Copenhagen: Copenhagen Business School Press.

Hjorth, Daniel/Steyaert, Chris (2003): 'Entrepreneurship Beyond (a New) Economy: Creative Swarms and Pathological Zones'. In: Steyaert, Chris/Hjorth, Daniel (eds.): New Movements in Entrepreneurship, London: Edward Elgar, pp. 286-303.

Hjorth, Daniel/Steyaert, Chris (2006): American Psycho/European Schizo: Stories of Managerial Elites in a Hundred Images. In: Gagliardi, Pasquale/Czarniawska, Barbara (eds.): Management Education and Humanities, Cheltenham: Edward Elgar, pp. 67-97. 
Hubbard, Phil/Hall, Tim (1998): 'The Entrepreneurial City and the "New Urban Politics"”. In: Hall, Tim/Hubbard, Phil (eds.): The Entrepreneurial City: Geographies of Politics, Regime and Representation. West Sussex: John Wiley, pp.1-27.

Landry, Charles (2000): The Creative City: A Toolkit for Urban Innovators, London: Earthscan.

Latham, Alan (2006a): 'Euro-Commentary: Anglophone Urban Studies and the European City: Some Comments on Interpreting Berlin'. In: European Urban and Regional Studies 13 (1), pp. 88-92.

Latham, Alan (2006b): 'Euro-Commentary: Berlin and Everywhere Else: A Reply to Allan Cochrane'. In: European Urban and Regional Studies 13 (4), pp. 377-379.

Latham, Alan (2003): 'Urbanity, Lifestyle and Making Sense of the New Urban Cultural Economy: Notes from Auckland, New Zealand'. In: European Urban and Regional Studies, 40 (9), pp. 1699-1724.

Latham, Alan (1999): 'Powers of Engagement: On being Engaged, being Indifferent; and Urban Life'. In: Area, 31 (2), pp. 161-168.

Lefebvre, Henri (1996/1968): 'Right to the City'. In: Kofman, Eleonore/Lebas, Elizabeth (eds.), Henri Lefebvre, Writings on Cities. Malden, MA and Oxford, UK: Blackwell, pp. 63-184.

Legates, Richard T. (2000): 'Expansive Overview of Cities'. In: Journal of the American Planning Association 66(2), pp. 201-202.

Locke, Robert R./Schöne, Katja E. (2004): The Entrepreneurial Shift, Cambridge: Cambridge University Press.

Luna-Garcia, Antoni (2008): 'Just another Coffee! Milking the Barcelona Model, Marketing a Global Image, and the Restoration of Local Identities'. In: Cronin, Anne M./Hetherington, Kevin (eds.), Consuming the Entrepreneurial City: Image, Memory, Spectacle. London: Routledge, pp. 143-160.

Lyle, Erick (2008): On the Lower Frequencies: A Secret History of the City, Berkeley: Soft Skull Press.

Lyotard, Jean-Francois (1984): The Postmodern Condition. Minneapolis: University of Minnesota Press.

Mace, Alan/Hall, Peter/Gallent, Nick (2007): 'New East Manchester: Urban Renaissance or Urban Opportunism?' In: European Planning Studies 15 (1), pp. 51-67.

Marshall, Tim (Ed.) (2004): Transforming Barcelona, London: Routledge.

Massey, Doreen B. (2005): For Space, London: Sage.

McNeill, Donald (2001): 'Barcelona as Imagined Community: Pasqual Maragall's Spaces of Engagement'. In: Transactions of the Institute of British Geographers 26 (3), pp. 340-352. 
Oswald, Philipp (2000): Berlin - Stadt ohne Form. Munich: Prestel Verlag.

Peck, Jamie (2005): 'Struggling with the Creative Class'. In: International Journal of Urban and Regional Research 29 (4), pp. 740-770.

Quilley, Stephen (2000): 'Manchester First: From Municipal Socialism to the Entrepreneurial City'. In: International Journal of Urban and Regional Research 24(3), pp. 601-615.

Rancière, Jacques (2004): The Politics of Aesthetics: The Distribution of the Sensible, London/New York: Continuum.

Robinson, Jenny (2006): Ordinary Cities, Abingdon: Routledge.

Soja, Edward W. (2000): Postmetropolis. Critical Studies of Cities and Regions, Oxford, UK and Malden, US: Blackwell.

Steyaert, Chris (2004): 'The Prosaics of Entrepreneurship'. In: Hjorth, Daniel/Steyaert, Chris (eds.), Narrative and Discursive Approaches in Entrepreneurship. Edward Elgar: London, pp. 8-21.

Steyaert, Chris (2006): 'Cities as Heterotopias and Third Spaces: The Example of ImagiNation, the Swiss Expo02'. In: Clegg, Stewart R./Kornberger, Martin (eds.), Space, Organizations and Management Theory. Malmö: Liber/Copenhagen Business School Press, pp. 248265.

Steyeart, Chris (2007): 'Of course that is not the whole (toy) story: Entrepreneurship and the cat's cradle'. In: Journal of Business Venturing 22, pp. 733-751.

Thrift, Nigel (2000): 'Afterwords'. In: Environment and Planning D: Society and Space 18 (2), pp. 213-255.

Thrift, Nigel (2005): 'But Malice Aforethought: Cities and the Natural History of Hatred'. In: Transactions of the Institute of British Geographers 30 (2), pp. 133-150.

Thrift, Nigel (2008): Non-Representational Theory: Space, Politics, Affect, London, UK and New York, US: Routledge.

Thrift, Nigel/Dewsbury, John-David (2000): 'Dead geographies - and how to make them live'. In: Environment and Planning D: Society and Space 18 (4), pp. 411-432.

Virilio, Paul (2005): City of Panic, Oxford: Berg.

Williams, Gwynda (2003): The Enterprising City Centre: Manchester's Development Challenge. London: Spon.

Zukin, Sharon (2008): 'Foreword'. In: Cronin, Anne M./Hetherington, Kevin (eds.), Consuming the Entrepreneurial City: Image, Memory, Spectacle. London: Routledge, pp. xi-xiii. 



\section{Kreatives Singapur}

BIRGit StÖBER, CAN SENG OOI

While we can leave Singapore's brand to develop organically over the years, we can also take the initiative to shape perceptions of Singapore ${ }^{1}$

Wie Singapur gerade in der westlichen Welt wahrgenommen wird, soll nicht dem Zufall überlassen werden, darin scheint im Inselstadtstaat große Einigkeit zu bestehen. Und vor diesem Hintergrund wird seit einigen Jahren ein ministerielles, administratives und institutionelles Governance Netzwerk errichtet, das Singapur assoziativ mit Begriffen wie Kreativität und Vielfältigkeit in Verbindung bringen soll.

Bisher wuchs Singapurs Ökonomie dank zielgerichteter Wirtschaftsprogramme bis Mitte des Jahres 2008 unvermindert und ließ das hochentwickelte Land mit seinen rund viereinhalb Millionen Einwohnern zu einem der bedeutendsten Wirtschaftsstandorte im Südostasiatischen Raum werden. Dieser Erfolg war vor allem dem Wachstum in der Elektronik- und ölverarbeitenden Industrie zu verdanken, seit einiger Zeit wird jedoch neben Finanzdienstleistungen und Tourismus auch wirksam auf die Creative Industries gesetzt. Diese Schwerpunktverlagerung ist zum einen dem rasanten wirtschaftlichen Aufschwung Chinas geschuldet, zum anderen lässt sich diese Kursänderung auf den Wunsch zurückführen, international auch in den Bereichen Lifestyle und Innovation eine bedeutende Rolle zu spielen.

1 Vgl. app.mica.gov.sg (Zugriff am 19.1.2009). 
Wesentlich mitverantwortlich für diese Kursänderung ist das Ministry of Information, Communication and the Arts (MICA), das mit einem umfassenden Katalog an Initiativen und Aktivitäten zur Änderung bzw. Verbesserung des singapurischen Rufs beitragen soll. „Multi-dimensional creativity - artistic creativity, business entrepreneurship and technological innovation - will be the new currency of success" heißt es auf der Homepage der Creative Community Singapore ${ }^{2}$, einer der Initiativen des Ministeriums.

Ebenso ist es das MICA, das für ein umfassendes Re-Branding Singapurs hauptverantwortlich zeichnet, mit dem der neue wirtschaftlichen Aufschwung sowohl nach innen als auch nach außen hin kommunizieren werden soll. Über Jahre hinweg hatte der Stadtstaat in der Außendarstellung auf seine multikulturelle Bevölkerung und die Mischung aus „exotischem Osten“ und „effizientem Westen“ gesetzt (Ooi 2004). Da jedoch Nachbarländer wie Malaysia, Thailand und China zunehmend auf ähnliche Bilder und Assoziationen setzen, schien die Entwicklung einer neuen Strategie angebracht. Zwar mag das offizielle Brand Singapur bislang als einen spannenden Ort beschrieben haben, doch ist der Stadtstaat bis vor kurzem eher eine kulturelle Brachlandschaft gewesen. Selbst die Behörden räumen ein, dass Singapur nur unzureichend in der Lage war, anspruchsvolle, kulturelle Aktivitäten anzubieten, die zum Beispiel hochqualifizierte ausländische Arbeitskräfte ansprechen und ins Land holen könnten (Yusuf/Nabeshima 2005). Und so soll die jüngste Branding-Initiative Singapur dazu verhelfen seinen „Ruf als quietschsauberer und stinklangweiliger Nachtwächterstaat gegen ein neues Image als lebenslustige, weltoffene, dynamische Metropole auszutauschen “3.

Singapur ist bekannt für strenge Regulierungen sowohl im sozialen als auch politischen Bereich. Nach wie vor können schon kleine Vergehen wie das Überqueren einer Straße bei Rot oder das Spucken im öffentlichen Raum bestraft werden. Doch deuten politische Erklärungen der vergangenen Jahre auf ein in Zukunft offeneres und toleranteres Singapurs hin. So wurde im Jahr 2003 aus dem Wunsch heraus den Stadtstaat zu einem unternehmerischen, attraktiven und kreativen Ort zu machen, der „Remaking Singapore Ausschuss“ eingerichtet. Viele der Empfehlungen des Ausschusses sind bereits implementiert, so z. B. die Förderung von akademischer Forschung im Bereich öffentliche Politik sowie die Abschaffung der obligatorischen „Vorab-Sicherheitsüber-

2 app.creativecommunity.sg (Zugriff am 19.01.2009).

3 Vgl. www.spiegel.de (Zugriff am 19.01.2009). 
prüfung" von Theater- und Filmmanuskripten. ${ }^{4}$ Kunst und Kultur zu fördern ist also nicht nur Teil der Bemühung die Kulturwirtschaft Singapurs zu entwickeln, sondern auch den internationalen Ruf zu ändern bzw. zu verbessern.

Von Regierungsseite wurden in diesem Zusammenhang vier Strategien entwickelt, die die „Brand Singapur Story“ mit Leben erfüllen sollen. So lockerte die Regierung in jüngster Zeit einige gesetzliche Regulierungen, um die kulturelle Szene zu beleben. Dies geschah trotz massiver Bedenken einiger Parlamentsmitglieder. Die damalige Wirtschaftsministerin Vivian Balakrishnan argumentierte jedoch öffentlich für die Lockerungen und zwar mit dem Verweis auf Richard Florida, demzufolge vor allem Orte, die Platz für Vielfältigkeit lassen, in der Lage sind nicht nur vermehrt Touristen, sondern auch - und gerade - Schlüsselfiguren für eine wissensbasierte Wirtschaft, kurz: die kreative Klasse, anzuziehen und zu fördern. „The larger lesson for us in Singapore is that we need to shift our mindset so that we can be more tolerant of diversity." 5

Eine weitere Strategie zur Umwandlung Singapurs in einen kulturell attraktiven Standort ist die, spektakuläre Großveranstaltungen wie Konzerte von internationalen Popstars und Blockbuster Ausstellungen im eigenen Land abzuhalten. Bei dieser Strategie wird der Begriff „Kultur“ insgesamt sehr weit gefasst, wie zum Beispiel im Fall der Ausrichtung des weltweit ersten Formel Eins Nachtrennen im Jahr 2008 sowie die für das Jahr 2010 geplanten ersten Olympischen Spiele für Jugendliche. Kritiker verweisen in diesem Zusammenhang auf die zu starke Fokussierung ,auf den ausgabefreudigen High-End-Markt ${ }^{\text {“6 }}$, der in Zukunft unter Druck geraten könnte.

Zusätzlich bemühen sich die singapurischen Behörden nicht nur um Engagement und Investitionen aus dem privaten Sektor, sondern auch darum von globalen und regionalen Organisationen aus den Bereichen Design, Telekommunikation, Pharmazie und dem Finanzwesen als Drehkreuz anerkannt zu werden. Mit den regionalen Hauptniederlassungen von MTV, Discovery, HBO und BBC nimmt Singapur im Medienbereich bereits eine prominente Position ein, doch ist damit nur ein Teil des offiziellen Plans abgedeckt, ein attraktives Umfeld für die creative industries zu schaffen. Um in den Bereichen Kunst und Kultur als respektierte Stadt zu erscheinen, wurden in den vergangenen Jahren zudem

4 Vgl. Singapore Government, 2004.

5 Vivian Balakrishnan in Singapore Parliament Hansard, 2004.

6 Vgl. www.spiegel.de (Zugriff am 19.01.2009). 
die Hafenfront architektonisch umfassend ausgestaltet sowie zahlreiche Kulturinstitutionen gegründet und eröffnet; im Jahr 1997 allein drei nationale Museen und im Jahr 2002 der Theater-Konzertsaal-Einkaufscenter-Komplex Esplanade - Theatres by the Bay. Außerdem soll der im Bau befindliche gigantische Gebäudekomplex Fusionopolis in Zukunft nicht nur der Mittelpunkt für Wissenschaft und Technologie, sondern auch für Medien und Kultur sein. Mit diesen urbanen, architektonischen Interventionen im Benjaminschen Sinne, soll dem Regierungsziel zufolge, der Stadtstaat nicht nur zum kulturellen Zentrum Südostasiens avancieren (Ooi 2007), sondern auch weltweit Beachtung finden.

Der in diesem Zusammenhang beobachtbare politische Prozess verläuft zweifellos Top-down gesteuert ohne etwa lokale Interessenvertreter wie z. B. Unternehmen, Ausbildungsinstitutionen oder Forscher einzubinden. Stattdessen werden Empfehlungen von ausgewählten Experten aus dem Ausland, vor allem England und Hongkong, nachgefragt. Hier können also international verzweigte Governance-Praktiken beobachtet werden, wie sie so nicht in Westeuropa zu beobachten sind.

Als ,,soft authoritarian“ (Chua 1995) wird das singapurische politische System oft charakterisiert und diese Art der Führung mag gerade bei einer so klaren Programmatik, wie sie das MICA seit einigen Jahren verfolgt, „,zweckdienlich“ sein. Bislang lässt sich noch kein wirkliches Fazit der singapurischen Strategie ziehen, doch lässt sich fragen, ob mit dieser Art der Governance langfristige Erfolge erzielt werden können, wenn es sich um kreative Prozesse handelt und nicht um die Herstellung von Elektronikbauteilen.

\section{Literatur}

Chua, Beng Huat (1995): Communitarian Ideology and Democracy in Singapore, London: Routledge.

Ooi, Can-Seng (2004): „Brand Singapore: The Hub of New Asia“. In: Morgan, Nigel/Pritchard, Annette/Pride, Roger (Hg..): Destination Branding: Creating the Unique Destination Proposition, London: Elsevier Butterworth Heinemann, S. 242-262.

Ooi, Can-Seng (2007): „The Creative Industries and Tourism in Singapore“. In: Richards, Greg/Wilson, Julie (Hg.): Tourism, Creativity and Development, London: Routledge, S. 240-251.

Singapore Parliament Hansard (2004): Singapore Parliament Hansard, Vol. 77, Session 1, Singapore: Singapore Parliament.

Yusuf, Shahid/Nabeshima, Kaoru (Hg.) (2005): „Creative Industries in East Asia“. In: Cities, 22, S. 109-122. 


\section{BRANDING/MARKETING}





\section{Orte, Städte und Kreativökonomien}

\section{als Brand}

\section{BIRGIT STÖBER, ARES KALANDIDES}

Geographische Einheiten wie z. B. Städte oder Regionen als brands oder Marken zu verstehen setzt einige Annahmen voraus, die eng mit einem „unternehmerischen“ Verständnis von städtischer Governance verbunden sind (Harvey 2001) und im Kontext von Globalisierungsdiskussionen von einer globalen, intra-urbanen Konkurrenz ausgehen (Cochrane/Jonas 1999). Zu diesen Annahmen gehören, dass Städte (im Folgenden auch stellvertretend für andere Raumeinheiten) als geschlossene, produktähnliche Einheiten behandelt und vermarktet werden können. Städte werden also heute ,zunehmend vor die Aufgabe gestellt, mit anderen Räumen in Konkurrenz zu treten, um wirtschaftsräumliche Vorteile in einer globalisierten Ökonomie zu erzielen“ (Mahnken 2003: 268). Vor diesem Hintergrund werden Stadtmarketing und -branding Strategien eingesetzt, um Städten Wettbewerbsvorteile zu verschaffen. Warum es jedoch nur bedingt möglich ist, räumliche Einheiten als Marken bzw. brands anzusehen und sie zu bewerben, also ihnen den Charakter von Waren zuzusprechen, wird in diesem Beitrag erläutert. Zudem wird der Text auf den Unterschied von Stadtmarketing- und Place BrandingAktivitäten eingehen. Das Konzept der „kreativen Stadt“ im konkreten Fall der Stadt Kopenhagen wird uns dabei als Beispiel dienen.

Schon seit Mitte der 1980er Jahre können wir in Europa eine Verbindung von Kultur, Kreativität und urbanen Räumen beobachten, und seit einigen Jahren wird diese Verbindung auch in Place Branding Kampagnen betont. Als gutes Beispiel kann hier die Etablierung des Konzeptes „Kulturhauptstadt Europas“ seit dem Jahr 1987 genannt werden. Kunzmann (2002: 187) zufolge kann die Verleihung des Titels „Kultur- 
hauptstadt Europas“ als „Trophäe im Stadtmarketing“ angesehen werden. Ähnliches Potenzial kommt dem UNESCO Netzwerk der „Creative Cities“ zu, um deren Mitgliedschaft sich Städte seit dem Jahr 2004 bewerben können. ${ }^{1}$

Die oft aufwendige Bewerbung um derartige internationale Auszeichnungen im Kulturbereich sowie die Ausrichtung von kulturellen Ereignissen begleiten und unterstützen Place Branding Aktivitäten mittlerweile in vielen europäischen Städten.

Während die gängige Stadtmarketing-Praxis - vor allem im Bereich der Investorenakquisition - seit Jahrzehnten auf der Vermittlung von quantitativen Daten bzw. harten Standortfaktoren wie Infrastruktur, Steuersätze, Arbeitskraftpotenzial, Höhe der Fördersummen, u. ä. basierte, ist seit einigen Jahren eine deutliche Schwerpunktverlagerung auf sogenannte weiche Standortfaktoren wie z. B. Standortprestige, Bildungssowie Erholungs- und Freizeitangebote zu beobachten. In diesem $\mathrm{Zu}-$ sammenhang erlangen die Bereiche Kultur und Kreativität eine zentrale Bedeutung. Diese Entwicklung kann insbesondere für Orte von Vorteil sein, die sich im strukturellen Umbruch befinden, hierbei sei an Sheffield, (Manchester) oder das Ruhrgebiet gedacht oder deren Attraktivität nicht oder nicht nur an harten Standortfaktoren festzumachen wie zum Beispiel im Fall von Berlin. Parallel zu dieser Entwicklung lässt sich eine Verschiebung der Strategien vom Stadtmarketing zum Stadt-Branding erkennen.

\section{Vom Place Marketing zum Place Branding}

Wir sehen einen deutlichen Unterschied zwischen Place Branding und Place Marketing Aktivitäten. Während Place Marketing eine Praxis ist, die schon seit vielen Jahrzehnten von Tourismus- und Investmentorganisationen angewandt wird, um auf Städte und Regionen aufmerksam zu machen, ist Place Branding ein eher neuer, strategischer Kommunikationsprozess, der eine größere Anzahl von Akteuren fordert und einbin-

1 Der Titel der „kreativen Stadt“, so wie er von der UNESCO mit den Unterbereichen, City of Design, City of Literature, City of Music, City of Cinema, City of Gastronomy und vieles mehr verliehen wird, soll nicht in erster Linie als Stadt-Branding-Thema dienen. Das „Creative Cities Network" der UNESCO wurde mit dem Ziel gegründet, die soziale, wirtschaftliche und kulturelle Entwicklung von Städten zu fördern. Die Städte, die sich für das Netzwerk bewerben, unterstützen die UNESCO-Mission für kulturelle Vielfalt. 
det als beim Place Marketing. Während beim Place Marketing die Außendarstellung dominiert, fordern Place Branding Aktivitäten den erweiterten Fokus auf die Einbindung und Mobilisierung der bereits ansässigen Bevölkerung. Dabei kommt dem Schaffen einer gemeinsamen Identität bzw. dem Bewusstsein für eine bestimmte Räumlichkeit und die Identität eines spezifischen Raumes, besondere Bedeutung zu. Auch konzeptionell unterscheiden sich beide Ansätze stark voneinander: „Branding zielt darauf, Assoziationen mit der Stadt zu schaffen; Assoziationen emotionaler, mentaler, psychischer Natur, weg vom funktional-rationalen Charakter von Marketingmaßnahmen“ (Kavaratzis 2008: 11, unsere Übersetzung). Dafür ist die Kommunikation der oben erwähnten nach wie vor als ,weich“ bezeichneten Standortfaktoren sehr dienlich. Denn sie schafft diskursiv genau die Assoziationen, die mit einem Ort in Verbindung gebracht werden. Solche Assoziationen können sowohl intern als auch extern auf gewisse Raumqualitäten hinweisen und einen Eindruck von Unverwechselbarkeit vermitteln.

Bei Place Branding Aktivitäten handelt es sich also um klar gebündelte und zielgerichtete Aktivitäten sowie strategische Allianzen, die als ein Mix aus schon bekanntem nach außen gerichtetem Standortmarketing plus einer nach innen gerichteten komplexen Image-Kampagne verstanden werden können (Stöber 2007).

So wird bei der jüngsten Ausschreibung, mit der die Investmentorganisation Copenhagen Capacity gemeinsam mit der Touristenorganisation Visit Copenhagen europaweit nach einem neuen Brand für die Stadt Kopenhagen suchen, besonders auf die Wichtigkeit der lokalen Verankerung der neuen Brands verwiesen. Dieser Fokus auf die Wohnbevölkerung geschieht vor dem Hintergrund, dass jeder einzelne Bewohner potenziell als ein ,walking talking advertisement“ (Winfield-Pfefferkorn 2005) betrachtet werden muss.

Charakteristisch für Place Branding Aktivitäten ist es, unattraktive oder unerwünschte Aspekte bewusst auszublenden oder zu negieren. Das Positive zu betonen und das Negative auszublenden, ist Morgan und Pritchard (Morgan/Pritchard 2004) zufolge die Strategie schlechthin beim Place Branding. Städtisches Branding lässt sich vor diesem Hintergrund als betörendes ,story telling“ bezeichnen, das darauf abzielt, die Rezipienten zu lehren, die Stadt in einer bestimmten Art und Weise zu sehen. Jensen (Jensen 2005: 12) bezeichnet diese Situation als eine dynamische Beziehung von „foregrounding“ und „backgrounding“, also als eine Inszenierung sowie ein Scharfstellen und Ausblenden von unerwünschten Aspekten.

Dennoch ist negative Publicity nicht zu vermeiden. Ganz entscheidend für Akteure bei Place Branding Kampagnen ist das Wissen, dass 
sie weder alle Facetten des Ortes noch alle Kommunikation über den Ort kontrollieren können. Diese Situation umschreibt Kotler (Kotler/Gertner 2002) mit dem Begriff ,uncontrolled communications“, der sich auf die Situation bezieht, dass formelle Marketingbotschaften von anderen Informationen wie z. B. Berichten aus den Medien, Erzählungen aus dem Freundeskreis usw. übertönt werden können.

Zudem ist Place Branding von einer besonders optimistischen Rhetorik geprägt, die in den meisten Fällen auf Einbindung statt Ausgrenzung setzt. Place Brands laden Menschen ein teilzuhaben an einer viel versprechenden Gemeinschaft, die sich manifestiert in Slogans, Ikonen, Architekturen und Narrativen. Dazu werden Berater eingebunden und Agenturen gegründet, die die jeweilige Stadt oder Region als Produkt mit einem eigenen Image und eigener Persönlichkeit bewerben. Hierbei ist nicht nur die Schaffung eines werbewirksamen Images $\mathrm{zu}$ beobachten, sondern auch ,ein umfassendes ,urban image“ mit politischer und sozialer Wirksamkeit" (Meyer zu Schwabedissen/Miggelbrink 2005: 18).

Ein weiteres Charakteristikum von Place Branding Aktivitäten ist das einer generellen Professionalisierung und Einbeziehung von privater Expertise sowie verstärktes Private Public Partnership Engagement. Städtisches Branding kann also als Teil einer stadtpolitischen Umorientierung gesehen werden und zwar hin zu flexibleren Formen der Marktprinzipien in der öffentlichen Politik, die sich mit dem Begriff „New Urban Management“" (Jensen 2005: 15) umschreiben lassen.

\section{Kreativität als Marke}

Kultur und Kreativität sind zu integralen Elementen von Planungsstrategien auf verschiedenen Ebenen geworden (Kalandides 2007). Eine deutliche Unterscheidung muss jedoch gemacht werden zwischen imagebildenden Strategien und den unmittelbarer auf wirtschaftliches Wachstum ausgerichteten Maßnahmen. Erstere betrachten Kreativität als Marke, als einen Zusatz zum, bzw. eine Assoziation mit dem Stadtnamen, während im zweiten Fall konkrete, materiellere Maßnahmen (z. B. Stadterneuerung, Firmenansiedlungspolitik) im Namen der Kreativität stattfinden. Versteht man Branding als eine integrative Herangehensweise die mentale und materielle Elemente beinhaltet (s. auch Kavaratzis 2008) so gehören beide Elemente dazu. Kreativität wird zu einer Art „Dachmarke“ unter der kommunikative und materielle Maßnahmen realisiert werden. Dabei wird den Kreativen eine doppelte Rolle zugeschrieben: zum einen als Vehikel zum Ziel und zum anderen als Zielgruppe von Stadtmarke- 
tingstrategien. Hier wird eine gewisse Tautologie der Kreativitätsdebatte deutlich: Kreative gehen dorthin, wo sich Kreative bereits befinden.

\section{Kreatives Kopenhagen}

Eine der entscheidenden Zielgruppen der bereits erwähnten zukünftigen Branding Kampagne für die Stadt Kopenhagen ist die Gruppe der Kreativen, von der erwartet wird, dass sie für die Stadt Kopenhagen wesentliche Wettbewerbsvorteile bedeuten kann. So wird von nationaler und kommunaler Seite ganz bewusst auf die Brand Kreatives Kopenhagen gesetzt. Auf Wunsch des dänischen Wirtschaftsministeriums, die Hauptstadt des Landes gezielt am globalen Spiel um allgemeine Aufmerksamkeit teilnehmen zu lassen, wurde im Jahr 2005 das Designfestival INDEX initiiert. Flankiert von einer internationalen Konferenz, Workshops und einer Preisverleihung für das beste Design „to improve life“, erhoffte man sich von dieser Initiative, die im Jahr 2007 zum zweiten Mal in Kopenhagen abgehalten wurde, weltweit positive Aufmerksamkeit und Anerkennung zu erreichen. Den Initiatoren zufolge soll Kopenhagen mit dieser Begebenheit als „kreative Stadt“ international zur Geltung gebracht werden. Und auch die Kopenhagener Bürgermeisterin unterstreicht diesen Wunsch, wenn sie sich folgendermaßen zitieren lässt: „INDEX ist äußerst wichtig bei unserer Bemühung, Kopenhagen als innovative, kreative, humane und attraktive Stadt zu branden“" (unsere Übersetzung) ${ }^{2}$.

Obwohl diese Initiative eine starke internationale Ausrichtung aufweist, geht es bei INDEX auch um die notwendige lokale „Bodenhaftung“, die für Branding Aktivitäten gefordert wird. So wurden zum Beispiel beide Male - 2005 und 2007 - alle nominierten Designobjekte zentral in der Innenstadt, dem Kongens Nytorv, ausgestellt und somit einer breiten Öffentlichkeit zugänglich gemacht.

Neben Design ist der Bereich Mode in den vergangen Jahren ebenfalls als wichtiger kultureller Aspekt entwickelt worden. Durch die Modemesse Copenhagen Fashion Week, die sich in nur wenigen Jahren zum größten Mode-Event in Nordeuropa entwickelt hat, erhält die Stadt international bedeutende Aufmerksamkeit die über die Modebranche hinausgeht. Einer Aufstellung der Dänischen Handelskammer zufolge ${ }^{3}$, nimmt die Copenhagen Fashion Week den fünften Platz auf einer Liste von Ereignissen ein, die den größten positiven Einfluss auf das interna-

2 www.indexaward.dk (Zugriff am 9.11.2008)

3 www.wonderfulcopenhagen.dk (Zugriff am 26.1.2009) 
tionale Image Dänemarks und Kopenhagens hatten. An erster Stelle rangiert das Hans Christian Andersen Jubiläumsjahr 2005, an dritter Stelle die Hochzeit des Kronprinzenpaares im Jahr 2004 und an 13. Stelle das Designfestival INDEX 2005. Da es sich bei der Fashion Week ebenso wie dem INDEX Festival um wiederkehrende Ereignisse handelt, geht die Dänische Handelskammer davon aus, dass gerade diese Begebenheiten eine wichtige Rolle für die längerfristige Entwicklung der kulturellen Marke Kopenhagen bedeuten.

\section{Diskussion}

An den vorangegangenen Ausführungen wurde deutlich, dass Place Branding weitaus mehr als die Entwicklung und Verbreitung eines Slogans und eines Logos ist und auch weitaus mehr als eine reine Marketingaktivität, vielmehr muss Place Branding als ein politischer Prozess betrachtet werden, der aufgrund seiner Komplexität nur eingeschränkt steuerbar ist und sowohl von verbalen als auch visuellen Repräsentationen entscheidend mit gestaltet wird (Stöber 2007). Diese Repräsentationen in Form von Texten, Bildern, Filmen usw. mögen zwar dem Anspruch genügen wollen, inklusiv, allumfassend oder sogar demokratisch zu sein, können aber dennoch nur ausgewählte Fragmente einer räumlich-sozialen Einheit darstellen. Dabei stellt sich die Frage nach der Urheberschaft bzw. nach der Rolle und Intention derer, die die jeweiligen Fragmente auswählen, verbreiten und damit die Wahrnehmung von Orten beeinflussen. Peter Van Ham (2002) zufolge kann Place Branding als eine ,postmoderne Variante von Identitäts-Formation“ mit großem visuellen Einsatz verstanden werden.

Dass Raum relational produziert wird, ist seit Lefebvres „La production de l'espace“ (1974) eine gängige Erkenntnis, die das räumliche Denken seit den 1970er Jahren verändert hat. Dies ist jedoch nicht dasselbe wie die Behauptung, Räume bzw. Orte verhalten sich wie Waren und können dementsprechend so vermarktet werden. Es ist nicht Ziel dieses Beitrags, diese These detailliert auszuführen ${ }^{4}$, doch sind drei Elemente für unsere Position von zentraler Bedeutung: Erstens, dass Räume nie fertig gestellt werden, sondern dass sie Ergebnisse von unterschiedlichen, oft konkurrierenden und in Frage gestellten sozialen Beziehungen sind. Auseinandersetzungen und Machverhältnisse werden

4 Für eine tiefere Kritik am Konzept der Stadt als Ware vgl. Kalandides 2006; Ashworth 2008; Philo/Kearns 1993; Colomb 2008. 
demnach zu einem konstitutiven Element des Raumes, der ohne sie nicht $\mathrm{zu}$ denken ist. Zweitens, der ersten Aussage folgend, dass heterogene Akteure durch diese Beziehungen in völlig unterschiedlicher Art und Weise miteinander verflochten sind und diese daraus resultierenden Konstellationen nicht als Einheit gedacht werden können. Und drittens, dass Räume auch untereinander in Relation stehen und sich gegenseitig beeinflussen, so dass klare Abgrenzungen bzw. Grenzziehungen nicht immer möglich sind (Massey 1994).

Letzteres hat deutliche Auswirkungen auf unser Verständnis von Standortwettbewerb: Um über intra-urbane Konkurrenz zu sprechen, muss die Stadt als klar abgegrenzte und definierbare Einheit betrachtet werden. Nur so können die abgeschlossenen Entitäten „Städte“ gegeneinander auftreten. Sprechen wir von der Stadt als administrativer Einheit, so ist diese Klarheit gegeben. Sobald wir jedoch die relationale Dimension von Räumen anführen, wird das Objekt „Stadt“ immer verschwommener.

Obwohl Slogans und Logos beim Place Branding nur eine untergeordnete Rolle spielen, darf die Bedeutung der Sprache nicht unterschätzt werden: Indem Räume mit einem Namen versehen werden, entsteht ein Bild mit mannigfaltigen Konnotationen. So ruft der Name „Berlin“ Bilder hervor, die sich bestimmt - selbst im Kontext der Kreativität - von denen unterscheiden, die mit dem Namen „Kopenhagen“ entstehen. Versucht man aber diese Konnotationen sprachlich zu fassen, so entstehen austauschbare Beschreibungen wie sie in dem Beitrag von Björn Böhning (in diesem Band) zu lesen sind: „Multikulturalität“, „Vielfalt“, „kreative Tradition“ usw. Die paradoxe Situation ist also, dass, indem nach der Differenzierung unter Städten gesucht wird, immer ähnlichere Bilder produziert werden. Die Kunst einer städtischen Brandingsstrategie wird also darin liegen, den konnotativen Unterschied zwischen der „kreativen Stadt Berlin“ und der „kreativen Stadt Kopenhagen“ zu vermitteln.

\section{Zusammenfassung}

Dieser Beitrag beschreibt Place Branding Aktivitäten als komplexe, zielgerichtete Aktivitäten und strategische Allianzen, die als ein Mix aus nach außen gerichtetem Standortmarketing sowie einer nach innen gerichteten umfassenden image Kampagne verstanden werden können.

Ein brand beinhaltet immer ein gewisses Versprechen, das eingehalten werden muss (Ashworth 2008). Die Entscheidung, eine Stadt als „kreativ“ zu bezeichnen und sie als solche zu kommunizieren, hat kon- 
krete Auswirkungen nicht nur in der Art der Wahrnehmung, sondern auch in der Ausrichtung der daraus resultierenden städtischen Governance. Akteure, Ziele, Maßnahmen und ihr Zusammenwirken bekommen eine andere Form als wenn der Fokus z. B. auf der „Stadt als Industriestandort" läge. Dabei bleibt es nicht nur bei praxisbezogenen Schwierigkeiten, sondern auch bei einer Reihe konzeptioneller Probleme, die in den vorangegangenen Ausführungen thematisiert wurden:

Erstens ist es zweifelhaft, ob räumliche Einheiten (darunter auch Städte) Charakteristika von Produkten enthalten und als solche vermarktet werden können. Jede Etikettierung einer Stadt, wie z. B. als „kreativ“, würde dem prozessualen Charakter der Raumproduktion nicht gerecht werden und wäre dementsprechend begrenzend.

Zweitens werden im Stadtmarketing die Beziehungen zwischen Räumen selten anders konzeptionalisiert als im Kontext der globalisierungsbedingten Konkurrenz. Dass Räume wesentlich komplexere relationale Systeme darstellen, hat Auswirkungen auf die Art wie wir Place Branding bzw. Place Marketing verstehen und implementieren. Auch Kreativität entsteht nicht in der Isolation sondern innerhalb der Wechselbeziehungen zwischen Räumen.

Drittens sind die ortsbezogenen Attribute die im Kern des Place Branding liegen wesentlich subtiler als oft angenommen. Obwohl wir Unterschiede zwischen Orten assoziativ wahrnehmen, ähneln sich plakative oder vereinfachende Darstellungen zunehmend und vermitteln den Eindruck einer Homogenisierung. So ebnet die beliebige Wiederholung der Kreativitätsmarke fundamentale Unterschiede zwischen Städten ein.

Es ist nicht ausreichend, bekannte, selbst erfolgreiche ProduktBranding-Strategien auf Räume zu übertragen. Räume werden nach eigenen Regeln konstituiert, die entsprechend konzeptionalisiert werden müssen.

\section{Literatur}

Ashworth, Gregory (2008): Can we, do we, should we, brand places? Or are we doing what we think and say we are doing? Plenary paper for the 1st International Conference „Marketing Cities: Place Branding in Perspective“, Berlin, Dezember.

Cochrane, Allan. D./Jonas, Andrew (1999): „Reimagining Berlin: World City, National Capital or Ordinary Place?“, In: European Urban and Regional Studies, 6(2), S. 145-164.

Colomb, Claire (2008): Staging urban change, reimaging the city: the politics of place marketing in the „New Berlin“ (1989-2004), Un- 
veröffentliche Disseration, (Ph. D.), University College London, The Bartlett School of Planning, London.

Harvey, David (2001): „From Managerialism to Entrepreneurialism: the Transformation in Urban Governance in Late Capitalism“. In: Harvey, David (Hg.): Spaces of Capital. Towards a Critical Geography. Routledge, New York, S. 345-368.

Jensen, Ole B. (2005): Branding the Contemporary City - Urban Branding as Regional Growth Agenda? Plenary paper for Regional Studies Association Conference „Regional Growth Agendas“ Aalborg.

Kalandides, Ares (2006): Fragmented Branding for a Fragmented City: Marketing Berlin. Paper presented at the „Sixth European Urban \& Regional Studies Conference“, Roskilde, Denmark.

Kalandides, Ares (2007): Marketing the Creative Berlin and the Paradox of Place Identity. Paper presented at the „XXVII Conferenza Scientifica Annuale“ of the „Associazione Italiana di Scienze Regionali“, Bozen, Italien.

Kavaratzis, Mihalis (2008): From City Marketing to Citys Branding. An Interdisciplinary Analysis with Reference to Amsterdam, Budapest and Athens. Unveröffentliche Dissertation, (Ph. D.), Rijksuniversiteit, Groningen.

Kotler, Philip and Gertner, David (2002): „Country as Brand, Product and Beyond: A Place Marketing and Brand Management Perspective“. In: Brand Management, 9 (4-5), S. 249-261.

Lefebvre, Henri (1974): La production de l'éspace, Anthropos, Paris.

Kunzmann, Klaus R. (2002): „Kultur, Wirtschaft und Raumentwicklung“. In: Kultur als Faktor der Stadt- und Regionalentwicklung in Europa. Informationen zur Raumentwicklung 4-5, Bonn: S. 185197.

Mahnken, Gerhard (2003): „Zwischen Eigensinn und Fremdbild: Regionales Binnenmarketing am Beispiel der metropolitanen Region Brandenburg/Berlin“. In: Raumforschung und Raumordnung 4, S. 268-277.

Massey, Doreen (1994): „Global Sense of Place“. In: Space, Place and Gender, Cambridge: Polity Press.

Meyer zu Schwabedissen, Frederike/Miggelbrink, Judith (2005): „Wo der Standort trompetet, geht die Freiheit flöten: Bilder interurbanen Wettbewerbs am Beispiel der Bewerbung Leipzig“ zur „Candidate City“ für die Olympischen Spiele 2012. In: Social Geography 1, S. $15-27$.

Morgan, Nigel/Pritchard, Annette (2004): Contextualizing destination branding. In: Morgan, Nigel/Pritchard, Annette (Hg.): Destination 
Branding. Creating the unique destination proposition, Oxford: Elsevier, S. 11-41.

Philo, Chris/Kearns, Gerry (Hg.) (1993): Selling Places. The City as Culture Capital, Past and Present, Oxford: Pergmanon Press.

Stöber, Birgit (2007): „Von ,brandneuen“ Städten und Regionen - Place Branding und die Rolle der visuellen Medien“. In: Social Geography, 2, S. 47-61.

Van Ham, Peter (2002): Branding Territory: Inside the wonderful worlds of PR and IR theory. In: Millennium, 31 (2), S. 249-269.

Winfield-Pfefferkorn, Julie (2005): The Branding of Cities. Exploring City Branding and the Importance of Brand Image, www. brandchannel.com (Zugriff am 31.1.2009). 


\title{
Die Bedeutung der Kreativwirtschaft für die Außenwerbung des Landes Berlin
}

\author{
BJÖRN BÖHNING
}

Im März 2007 hat das Land Berlin mit der Imagekampagne „be Berlin“ neue Wege beschritten. Mit der Markenpositionierung „Stadt des Wandels" hat die Stadt bewusst auf die Entwicklung und Veränderung der Metropole seit 1990 gesetzt. Als einzige Stadt in der Welt kann Berlin mit seiner teilungsbedingten Historie für sich glaubwürdig und klar reklamieren, die Stadt des Wandels zu sein. Dabei hat sich seit 1990 ein fundamentaler Wandel von Stadtgesellschaft und Stadtökonomie vollzogen, der bis heute spürbar ist. Schmerzhaften Prozessen des Wandels folgten prosperierende Zeiten, die eine neue, veränderte Metropole andeuten, die noch lange nicht zur vollen Blüte gekommen ist.

Denn seit 1990 hat Berlin die Hälfte seiner Industriearbeitsplätze verloren. Und das nicht nur im Ostteil der Stadt. Allein in den tradierten Produktionsstandorten Westberlins ca. $45 \%$ der Arbeitsplätze im Produktionssektor weggefallen. Die stark subventionierten West- und OstBerliner Arbeitsplätze sind nach der Wende in atemberaubender Geschwindigkeit verschwunden. Rund 200.000 Industriearbeitsplätze hat Berlin seit 1991 verloren, rund 60.000 im Baubereich sowie etwa 90.000 im Handel- und Gaststättengewerbe.

Eine ähnliche - fast parallele - Entwicklung erlebte die Stadt in Bezug auf den Wandel der Bevölkerung. Rein statistisch gesehen hat sich bis heute in Berlin die Hälfte der Bevölkerung ausgetauscht. Etwa anderthalb Millionen Menschen kehrten der Stadt den Rücken oder zogen - auch und gerade als Folge des Umzugs des Regierungssitzes - neu in die Hauptstadt. Insbesondere viele junge und jüngere Menschen aus Deutschland, Europa und der Welt fühlten sich von der neuen Metropole 
im Herzen Europas angezogen. Berlin ist heute im internationalen Vergleich eine überaus junge Stadt. Zuletzt stieg die Zahl der 18- bis 35Jährigen in der Stadt um jährlich 20.000 an. Damit veränderte sich Berlin nicht nur rein demografisch, sondern auch kulturell. Junge Menschen brachten neue Lebensstile und Lebensweisen in die Stadt, nutzten die Freiräume der unfertigen Stadt für kreative Spielarten und Kombinationen von Arbeiten, Leben und Wirtschaften. Berlin strahlte (und strahlt) Offenheit aus, die Neues ermöglicht und kreative Ideen und Innovationen anregt. Dieses ,produktive Chaos“ sorgte dafür, dass die Stadt zum Anziehungspunkt für die kreativen Pioniere neuen Arbeitens und Wirtschaftens geworden ist.

Während allenthalben noch vom Wandel des Normalarbeitsverhältnisses als Anzeichen einer neuen Wirtschaftsweise sinniert wurde, vollzog sich diese Entwicklung in Berlin heimlich, still und leise in einem realen und mit hoher Eigendynamik ausgestatteten „Feldversuch“. Die Kreativwirtschaft eroberte sich die Räume links und rechts der Spree und begann erst langsam, dann immer schneller, Werte zu schöpfen und Wachstum zu generieren. Wie so oft wurde die Krise der alten Ökonomie zu einer Chance für die neue Wirtschaft. In Berlin vollzog sich dieser Wandel erst unterhalb der Oberfläche, heute zunehmend koordinierter und durch die Politik strategisch gefördert. Im Wandel zeigte sich, dass der radikale Einbruch der industriellen Landschaft der nunmehr wiedervereinigten Stadt, Pflänzchen neuer Ideen und Unternehmungen schneller zur Geltung und zum Sprießen brachte. ${ }^{1}$

\section{Die Kreativwirtschaft in Zahlen}

Heute trägt die Kreativwirtschaft zu einem Fünftel zum Wachstum Berlins bei. $^{2}$ Im Jahr 2005 erwirtschafteten 24.500 zumeist kleine und mittelständische Unternehmen in der Kreativwirtschaft einen Umsatz von

1 Dem Autor käme es nicht in den Sinn, dabei die sozialen Verwerfungen dieser Entwicklung aus dem Blick zu verlieren. Dies wäre jedoch Ansatzpunkt einer weiteren, thematisch anders orientierten Abhandlung.

2 Zum Cluster Kreativwirtschaft werden im „Kulturwirtschaftsbericht Berlin 2005“ folgende Bereiche gezählt. Im Bereich Medien: Marktforschung, Musik, Film \& TV, Buch- und Pressemarkt, Werbung/PR; im engeren Bereich der Kreativwirtschaft: Darstellende Kunst, Kunstmarkt, Architektur und Kulturelles Erbe; sowie im Bereich IT: Software/Multimedia, Internet/TK, Nachrichtentechnik, Hardwareberatung, DV-Dienste. Die folgenden Zahlen beziehen sich auf diese Quelle (Senatsverwaltung für Wirtschaft, Technologie und Frauen). 
$16 \%$ am Bruttoinlandsprodukt der Berliner Wirtschaft. In der Kreativwirtschaft sind ca. 160.000 Menschen sozialversicherungspflichtig beschäftigt. Zählt man zudem geringfügig Beschäftigte und Selbständige hinzu, kommt Berlin auf knapp 200.000 Menschen, die in der Kreativwirtschaft der Stadt arbeiten und ihr Einkommen daraus beziehen. Damit nimmt Berlin im internationalen Vergleich eine Spitzenposition ein.

Das Cluster steht für überdurchschnittlich hohe Wachstumsraten. Seit dem Jahr 2000 sind rund 4.000 neue Unternehmen dazugekommen. Das ist ein Plus von $19 \%$, die Umsätze sind im gleichen Zeitraum um etwa $22 \%$ (über 3,7 Mrd. Euro) gestiegen. Im bundesdeutschen Vergleich der Kreativstandorte (München, Hamburg, Köln) hat Berlin damit die höchsten Zuwachszahlen beim Umsatz und bei der Anzahl neuer Unternehmen.

\section{Die Kreativwirtschaft in der Imagekampagne "be Berlin“"}

Gerade weil in Berlin die Herstellung sozialer Kohäsion über materielle Zuflüsse allein nicht sichergestellt werden kann, setzt die Stadt auf die Förderung und Aktivierung endogener Potenziale, auch der Kulturellen. Die Bürgerinnen und Bürger selbst bilden das Kapital der Stadt und gestalten den Wandel positiv mit. Sie sind das Bild der Stadt. „be Berlin“ setzt ganz bewusst auf die Bildung einer aktiven Stadtgesellschaft, auf die Mitarbeit am sozialen und ökonomischen Fortschritt der Stadt. Gleichzeitig setzt die Kampagne den vielen engagierten Menschen - im Großen wie im Kleinen - eine Denkmal. Denn ohne sie wäre die Stadt arm. Auch unter den Akteuren in Politik und Verwaltung ist dieses Bild der Notwendigkeit einer aktiven Stadtgesellschaft heute weit verbreitet.

Talente, Technologie und Toleranz - so lautet das Credo Richard Floridas für erfolgreiche Metropolen in der Globalisierung. Die „Creative Industries“ spielen bei dieser Positionierung eine erhebliche Rolle sowohl für das reale Wirtschaftswachstum der Städte als auch für deren „Image“. Die Metropolen befinden sich heute in einem Wettbewerb um Fachkräfte aus aller Welt, die in ihren Forschungslaboren und Arbeitsstätten Innovationen und Produkte für die Welt denken und erarbeiten. Auch Berlin muss sich diesem Wettbewerb stellen, will es weiter ein international anerkannter und bedeutender Wissenschaftsstandort mit Potenzial für mehr Wachstum bleiben. Dabei gilt es nicht die Fehler anderer Städte $\mathrm{zu}$ wiederholen. Nicht selten sind es peinlich anmutende Versuche einzelner Standorte sich mit einem freien Theater und zwei Strandbars als kreativer Standort zu profilieren. Nicht nur bei Image- 
kampagnen ist Glaubwürdigkeit ein unschätzbarer Wert. Berlin kann diese Glaubwürdigkeit für sich beanspruchen und nutzen. Denn die kreative Landschaft Berlin hat spezifische Profile, die sich die Imagekampagne zu eigen macht:

- Als Hauptstadt und Metropole hat Berlin das urbane Umfeld, das für die Entwicklung der Kreativwirtschaft von entscheidender Bedeutung ist. In nahezu allen europäischen Ländern sind die Schlüsselbranchen der Kultur- und Medienwirtschaft in der jeweiligen Hauptstadtregion konzentriert. Durch die wirtschaftliche Dichte und Netzwerke in Berlin wird die Entwicklung kreativer und innovativer Milieus begünstigt.

- Als Stadt der kulturellen Vielfalt hat Berlin im europäischen Vergleich ein unverwechselbares, spartenübergreifendes und vielfältiges kulturelles Angebot im Bereich der populären Kultur und der Hochkultur.

- Berlin ist eine Stadt des Kulturtourismus mit steigender Tendenz. Die kulturellen Leuchttürme der Stadt ziehen Touristen aus aller Welt an - nicht wenige davon werden in der Hauptstadt heimisch.

- Berlin ist eine Stadt der kreativen Talente. Berlin hat als Forschungsund Wissenschaftsstandort internationale Bedeutung und globales Renommee. Mit ca. 140.000 Studierenden, davon allein 15.000 in den medien- und kommunikationswissenschaftlichen Studiengängen, nimmt Berlin einen europäischen Spitzenplatz ein.

- Berlin ist ein digitaler Standort an der Schnittstelle zwischen Wissenschaft und Wirtschaft. Als einer der innovativsten Standorte Europas hält Berlin entscheidende Kreativ- und Forschungskapazitäten vor.

- Berlin ist ein bedeutender Messe- und Festivalstandort, von der Internationalen Funkausstellung bis zur Berlinale.

- Und nicht zuletzt ist Berlin ein Schmelztiegel für Menschen unterschiedlicher Herkünfte. 13 Prozent aller Berliner sind Ausländerinnen oder Ausländer. Die Stadt lebt die kulturelle Vielfalt, und betrachtet sie als Chance, nicht als Gefahr.

Das Image von Berlins Kreativwirtschaft ist nach einer Studie über die Creative Class der Stadt vor allem von den Aspekten Kreativität, Kultur und Internationalität geprägt. Diese Faktoren gilt es auch im Rahmen der Imagekampagne hervorzuheben (Orco Germany/Berlin-Partner (2008). Mit der Positionierung ,be Berlin“ kommuniziert die Stadt gezielt kleine und große Erfolgsgeschichten aus Berlin. Sie macht deutlich, dass Ideen und deren Umsetzung Erfolg und Wachstum schaffen. Sie gestalten die Stadt, die so hervorragende Rahmenbedingungen für die Kreativwirt- 
schaft wie nirgendwo sonst: Ein hohes Maß an Freiräumen der Stadtentwicklung, günstige Mieten und Lebensbedingungen, eine kreative Kultur- und Popszene, einen international bedeutender Film- und Medienstandort und nicht zuletzt eine etablierte Wissens- und Innovationslandschaft. Nichts macht diesen Umstand deutlicher als die Geschichte der Rütli-Kids, die aus Kreativität, kultureller Vielfalt und Ideenreichtum, eine Chance für sich und ihr soziales Umfeld entwickelt haben:

Noch vor zwei Jahren sorgte die Rütli-Schule für Negativschlagzeilen in ganz Deutschland. Krawalle, tätliche Übergriffe auf Lehrer und Schüler, Wachschutz vor den Schultoren. Rütli war zum Symbol geworden für ein Bildungs- und Sozialsystem, das hilflos war angesichts unbewältigter Probleme. Doch durch vielfältiges Engagement ist es in den letzten Jahren gelungen aus dieser Schule eine Erfolgsgeschichte zu machen: Im Umkreis der Schule entsteht ein 50.000 Quadratmeter großer Campus, der den Kindern der Umgebung von der Kita bis zum Schulabschluss gute Bildungschancen bieten soll. Und seit zwei Jahren entwerfen Schüler der Rütli-Schule im Kunst- und Arbeitslehreunterricht eigene Motive und Logos für T-Shirts und Sweatshirts. Die Schülerinnen und Schüler drucken ihre Entwürfe auf die Textilien und verkaufen sie anschließend im eigenen Online-Shop. Unter anderem mit dem RÜTLIWEAR-Projekt ist es den Jugendlichen gelungen das Image ihrer Schule gehörig aufzupolieren. Voller Stolz tragen sie heute das Rütli-Logo. Man kann sagen, Rütli ist nicht mehr als Negativschlagzeile zu lesen, sondern auf der Straße, auf T-Shirts, in Berlins Kult- und Szeneläden. Aus einer kreativen Idee sind mittlerweile Arbeitsplätze und Umsätze geworden. Ein besseres Beispiel, dass dort, wo Kreativität, Talente und Toleranz zusammenkommen, Wachstum entsteht, und zwar sozialer wie ökonomischer Fortschritt, gibt es nicht.

Im Rahmen von „be Berlin“ werden diese und andere Erfolgsgeschichten, wo aus Kreativität und Innovationen ein ökonomischer wie gesellschaftlicher Mehrwert entstanden ist, strukturiert kommuniziert. Die Imagekampagne hat Beispiele geliefert, wo Menschen aus ihrer Kreativität Produkte und Arbeitsplätze geschaffen haben. Dies hat nicht nur die Bürgerinnen und Bürger sowie ihre Ideen profiliert, sondern hat auch die „Marke“ Berlin als Standort der Kreativen und der Kreativwirtschaft gestärkt.

Berlin setzt darauf, dass Kreative aus aller Welt die Stadt anziehend finden und in der Stadt arbeiten, wohnen und leben. So hat das Land Berlin kürzlich entschieden, die Zahl der Studienplätze nochmals aufzustocken. Denn immer wenn junge Kreative in die Stadt kommen, werden auch neue Potenziale für Wissenschaft, Forschung und Wachstum erschlossen. Hier entstehen Netzwerke, die zu einer Stärkung des Clusters 
Kreativwirtschaft beitragen. All dies zeigt: Wo überraschend unterschiedliche Disziplinen zusammenkommen, wo Netzwerke und Kontakte kultiviert werden, wo die Wirtschaftspolitik nicht kurzatmig ist, wird Wissen und Kreativität Arbeit und Wohlstand schaffen. „be Berlin“ verweist darauf und bietet der erfolgreichen Kreativwirtschaft einen kommunikativen Rahmen. Zugleich werden gezielt Begegnungen unterschiedlicher Disziplinen initiiert. Nicht zuletzt ist die Kampagne aber auch eine Wertschätzung der Politik ihnen gegenüber und das Signal: Ihr werdet gebraucht!

Die Berlin-Studie der Hertie-Stiftung gibt diesem Ansatz recht. Das Lebensgefühl der Berliner zeugt von einem großen Stolz auf Ihre Stadt: Knapp $90 \%$ aller Berlinerinnen und Berliner leben sehr gern oder gern in ihrer Stadt. $70 \%$ würden ihre Stadt als Wohnort weiterempfehlen. Das ist ein starkes Statement für den Ansatz der Imagekampagne ,be Berlin“.

\section{Ausblick}

Die Kreativszene der Stadt ist schon heute für das Image Berlins von unschätzbarem Wert. Berlin wird alles daran setzen, dieses Image zu verstärken. Trotz aller Euphorie über diese imageträchtige Branche und medialer (Eigen-)Darstellung der Kreativwirtschaft ist aber Nüchternheit angesagt. Schnell werden Einzelfälle und -berichte zu einer gesamtstädtischen Bedeutung hochstilisiert, ohne dass eine langfristige Entwicklung dahinterstünde. Deshalb wird das Land neben der Imagekampagne gleichzeitig durch eine gezielte und nicht kurzatmige Wirtschaftsförderung den Cluster Kreativwirtschaft nachhaltig fördern und ausbauen.

Im Jahr 2009 wird Berlin international für sich werben. Auch hier will Berlin sein Image als kreativer Standort ausbauen. Denn international wird Berlin - zu aller Überraschung - immer noch mit den typisch deutschen Tugenden, ordentlich, bürokratisch, fleißig und auch ein wenig langweilig, gleichgesetzt. Diese Ausgangslage bietet aber auch die Gelegenheit, das Image der Stadt als Standort der Kreativwirtschaft mit gezielten Provokationen und Überraschungen zu kommunizieren. Berlin soll international ,the place to $b e^{\text {" werden. }}$

\section{Literatur}

Orco Germany/Berlin-Partner (2008): Creative Class in Berlin - Studie über Branchenstrukturen und Standortverhalten der Kreativwirtschaft, Berlin: Berlin Partner. 


\section{URBAN Governance}





\section{Der lokale Staat als Akteur im Feld kreativer Nischenökonomien}

HANS-JOACHIM BÜRKNER

\section{Politik und kulturelle Nischenökonomien: Merkmale einer unsteten Beziehung}

Es ist nicht zu übersehen: Lokale kreative Ökonomien können sich nur selten im Glanz offizieller staatlicher Förderung und arrivierter Zurschaustellung sonnen. Den Mainstream-Segmenten der anerkannten Hochkultur sowie der kommerziellen Popkultur steht eine Vielzahl heterogener Akteure der alternativen Kulturproduktion gegenüber. Diese Akteure besetzen als Culturepreneurs bzw. kulturelle Unternehmer meist ausgesprochene ökonomische Nischen (Lange 2007), und zwar abseits der etablierten globalen Kreativökonomien (vgl. Krätke 2002). Diese Nischen zeichnen sich durch schmale Marktsegmente, eine hoch spezialisierte Nachfrage, geringe Profitorientierung der Unternehmen, geringe Kostendeckung und prekäre Arbeitsverhältnisse aus.

Waren derartige Nischen noch vor wenigen Jahrzehnten gut überschaubar, so ist in neuerer Zeit aufgrund von Globalisierungsprozessen Bewegung in die jeweiligen Szenerien gekommen. Den etablierten Akteuren setzen leere öffentliche Kassen und neue Konkurrenzen aus den „Billiglohnländern“ des Hochkultur-Business zu. Ein scharfer Wettbewerb um schwindende materielle Ressourcen und Arbeitsplätze erzwingt häufig Strategien der zusätzlichen Existenzsicherung, die bis in ökonomische Nischen hinein reichen können. Die ,alternativen“ Akteure erschließen aufgrund der globalen Hybridisierung des Konsumentengeschmacks sowie der beschleunigten Kommerzialisierung vormals regio- 
nal erstellter und vermarkteter Kulturprodukte zwar immer wieder neue Nischen, die Originalität und Exklusivität versprechen; zugleich werden jedoch die Produktionsbedingungen innerhalb dieser Nischen nicht zuletzt aufgrund stark verringerter staatlicher Förderung immer prekärer.

Neu ist auch die zunehmende politische Aufmerksamkeit, die diese Nischen derzeit erfahren. Lange Zeit hatten sie ein wenig beachtetes Eigenleben geführt. Sie wurden meist nur dann sichtbar, wenn sie massenmedial verwertbare Produkte hervorbrachten. Erste zaghafte Versuche der Annäherung an die neuen urbanen Szenerien der kulturellen Nischenproduktion (z. B. der elektronischen Popmusik) sind von stadtpolitischer und staatlicher Seite überwiegend zu Beginn dieses Jahrtausends zu verzeichnen gewesen - eine ganze Dekade, nachdem die betreffenden städtischen Subkulturen bereits intensiv an der Ausgestaltung und Weiterentwicklung ,,ihrer“ Nischen gearbeitet hatten. Schlagworte wie „,neue Steuerungsformen“ und „Governance“, die in diesem Zusammenhang fallen, deuten bereits an, dass es hier selten um rein administrative oder planerische Aufgaben geht. Vielmehr scheinen neue öffentliche Umgangsweisen mit den jeweiligen Phänomenen provoziert zu werden - sei es, um mit dem hohen Tempo der soziokulturellen und ökonomischen Entwicklungsprozesse Schritt halten zu können, sei es auch nur, um der Fragilität und Flüchtigkeit der jeweils entstehenden Strukturen Rechnung zu tragen. Dabei geht es um Annäherungen, Verständigungen und Aushandlungsformen zwischen teilweise antagonistischen Denkweisen und Handlungsformen. Der kulturellen Definitions- und Gestaltungsmacht des Staates steht dabei die gegenkulturelle Praxis des Untergrunds ebenso gegenüber wie die Logik der Wertzuweisung profitorientierter Marktproduktion. Ausgesprochen spannend in diesen Annäherungsprozessen ist der Umstand, dass es zu gemischten Akteursstrukturen zwischen Staat und Zivilgesellschaft kommt.

Im Folgenden werden die Herausforderungen für Politik und Governance, die von der Entwicklung städtischer Nischenökonomien ausgehen, am Fall älterer, im Globalisierungsprozess unter Druck geratener, kulturökonomischer Nischen exemplarisch betrachtet. Dabei wird zunächst grundsätzlich nach dem Verhältnis von Staat, Stadtpolitik und Nischenökonomien gefragt, bevor die besondere Frage nach den darin enthaltenen sozialräumlichen Komponenten aufgeworfen wird, die durch die sozialwissenschaftliche Raumforschung vermehrt thematisiert werden. Am Fall der Produktionsbedingungen für die Bereiche Neue Musik und Jazz und ihrer Thematisierung durch die Stadtpolitik in Berlin werden dann grundlegende Kommunikationsprobleme und Schwierigkeiten der Entstehung subkulturadäquater Steuerungsstrukturen auf- 
gezeigt. Abschließend werden Optionen kontextbezogener Steuerungsformen rund um das Phänomen kulturökonomischer Nischen skizziert.

\section{Das Beziehungsfeld zwischen Staat, Stadt und Nischenökonomien}

Wenn von mehrseitigen Beziehungsgeflechten zwischen Staat, Stadtpolitik und kulturellen Nischenökonomien die Rede ist, so muss berücksichtigt werden, dass in den Kommunikationsprozessen sehr unterschiedliche, strukturell verankerte Sichtweisen und Interessen zum Ausdruck kommen. Aufgrund der Heterogenität der strukturellen und sozialen Kontexte, denen die Akteure zugeordnet sind, sind in der Kommunikation jeweils „Übersetzungshilfen“ erforderlich.

Strukturell bedingte Interessendivergenzen leiten sich einerseits aus den Aufgaben und institutionellen Selbstverständnissen staatlicher und stadtpolitischer Organisationen her, andererseits aber auch aus den ökonomischen Strukturen und der sozialen Organisation der Kreativwirtschaft. An dieser Stelle ist eine begriffliche Unterscheidung in „Staat“ und „Stadtpolitik“ notwendig, um die Akteursdimension der diskutierten Steuerungsformen hinreichend präzise entfalten zu können. Obwohl in der angloamerikanischen Stadtliteratur traditionell vom „lokalen Staat“ die Rede ist, wenn lokale Politik und Administration gemeint sind (Cockburn 1977), reicht dies im Kontext kultureller Nischenökonomien zur Akteursbeschreibung nicht aus.

Stadtpolitische Akteure erfüllen hier nicht nur „Regierungsaufgaben“, sondern kommen auch als Partner bei der Ausgestaltung struktureller Rahmenbedingungen für die Entwicklung lokaler kulturökonomischer Nischen ins Spiel - etwa als Mitglieder intermediärer Organisationen wie z. B. privater Kulturinitiativen, Förderkreise usw. Demgegenüber sind staatliche Organisationen eher mit der Gestaltung allgemeiner Rahmenbedingungen befasst, die entweder indirekt (z. B. über die Verursachung von Sparzwängen für die Kommunen) oder direkt (z. B. über die fokussierte Bereitstellung von Fördermitteln für kulturelle Zwecke) auf die in Frage stehenden Nischen und die Handlungsspielräume ihrer Akteure einwirken.

Stadtpolitik und städtische Administrationen haben ihr Verhältnis zu Stadtökonomie und -kultur in der Vergangenheit hauptsächlich entlang sektoraler Grenzziehungen definiert. Dies kommt nicht nur in den traditionellen Zuständigkeiten der Ressorts (Wirtschaft, Kultur, Soziales, Gesundheit usw.) zum Ausdruck. Es äußert sich auch in den enormen Schwierigkeiten politischer Akteure bei dem Versuch, den hybriden 
Charakter kultureller Nischenökonomien zu verstehen - irgendwo zwischen originärer Erfindung, materieller Produktion, kulturellem Ereignis, spezifischen Formen des Sozialen und öffentlich sichtbaren politischen Implikationen der jeweiligen kulturellen Artefakte. So nehmen diese Nischen beispielsweise häufig den Charakter sozialer Ökonomien an, d. h. sie erfüllen über die ökonomische Funktion hinaus soziale und kulturelle Funktionen, die an lokale Kontexte, soziale Netzwerke, Szenen und Milieus gebunden sind. Diese besondere Beschaffenheit ökonomischer Nischen befindet sich nur teilweise im Blickfeld staatlicher Akteure. Abgesehen davon, dass es zum Problem der Hybridität dieser Nischen bislang nur wenige wissenschaftliche Erkenntnisse gibt, ist auch der einschlägige Informationsstand in den lokalen Öffentlichkeiten gering. Selbst kompetente Kulturpolitiker werden daher dazu verleitet, aus der vermeintlich geringen Wertschöpfung innerhalb der Kulturproduktion auf eine geringe Relevanz der jeweiligen Nischen für die Stadtentwicklung zu schließen.

Die sektorale Perspektive auf kreative Nischenökonomien beeinflusst auch die Frage nach geeigneten städtischen Steuerungsformen. Diese werden nämlich tendenziell an der Dimension „Hierarchie“ orientiert. Vorsichtige bis neugierige Kontaktaufnahmen von Politik, Verwaltung, Planung und Wirtschaftsförderung mit kulturökonomischen Nischen folgen daher zunächst bewährten Verwaltungsroutinen und Repräsentationslogiken. Im Vordergrund stehen Fragen wie: Welchen imagepolitischen Gewinn erhält die Stadt durch diese Ökonomien? Müssen sie gefördert werden, um einen entsprechenden politischen Mehrwert zu produzieren? Wie viel Geld kosten geeignete Fördermaßnahmen? Treten die Akteure als anspruchsvolle Antragsteller in Erscheinung, und wenn ja, wie häufig? Treten sie darüber hinaus als Lobbyisten und pressure groups auf, die Politik, Planung und Verwaltung unter Legitimierungsdruck setzen? Produzieren sie Kosten, indem sie auf der einen Seite bestimmte Stadtentwicklungsprozesse forcieren (z. B. den sozialräumlichen Umbruch von Szenequartieren), auf der anderen Seite Entwicklungen blockieren (z. B. städtebaulichen Aufwertungsprozessen im Wege stehen)? Unter dem Eindruck derartiger, strukturell und institutionell vorgeformter Fokussierungen entwickeln sich neue Steuerungsformen, die stärker auf interdisziplinären Netzwerken und marktnahen Kommunikationsformen basieren, eher zögerlich. Sie stellen insbesondere für die Akteure aus städtischer Politik und Verwaltung ausgesprochene Experimentierfelder dar, deren politische Relevanz angesichts der wechselnden Gesprächspartner und der flüchtigen Entwicklungen auf Seiten der beteiligten Subkulturen und ,ihrer“ Nischen immer wieder leicht in Frage gestellt werden kann. Genau hier scheinen sich die bislang größ- 
ten Divergenzen bei der Ausgestaltung lokaler Governance-Formen und -prozesse aufzutun. Der von der Politik entwickelte utilitaristische Anspruch auf die Formulierung neuer stadtpolitischer Aktivposten und Image-Zugewinne einer Stadt kollidiert zwangsläufig mit einer auf die Ermöglichung kreativer Erfindungsprozesse und der materiellen Existenzsicherung ausgerichteten Interessenlage der Nischenakteure.

\section{Kreative Nischenökonomien aus der Sicht einer sozialwissenschaftlichen Raumforschung}

Jüngere sozialgeographische Perspektiven auf die Milieus und Szenen der Wissensökonomie und der Kreativwirtschaft haben die sozialräumlichen Aspekte einer Stadtentwicklung thematisiert, die durch subkulturelle Ausdifferenzierungsprozesse zunehmend beeinflusst wird. Dabei sind zum einen Fragen der sozialen Konstruktion von Raum (auch physisch konnotierter städtischer Räume) samt der symbolischen Orientierungsfunktionen von Räumen und Orten für die jeweiligen Milieus behandelt worden (Bürkner 2004; Conell/Gibson 2003; Lange 2006; Lange 2007), zum anderen Probleme der gesamtstädtischen und stadtteilbezogenen Steuerung von Branchenentwicklungen, milieuspezifischen Infrastrukturen und Freizeitgelegenheiten, Kommunikationsorten usw. (Scharenberg/Bader 2005; Mager 2007). In Bezug auf die hier zur Debatte stehenden Nischenökonomien können mit einer raumbezogenen Forschungsperspektive zwei Fragenkomplexe bearbeitet werden, zu denen derzeit erheblicher Erkenntnisbedarf besteht: 1. die Frage nach den strukturellen Ausdifferenzierungsprozessen städtischer Nischenökonomien und 2. die Frage nach den Raumkonstrukten und Raumbezügen, die von staatlichen bzw. stadtpolitischen Akteuren einerseits und privatwirtschaftlichen Nischenakteuren anderseits formuliert werden.

1. Zur Frage der Strukturierung kreativer Nischenökonomien in urbanen Kontexten: Nischenökonomien, so lautet die Basisvermutung, sind auf die Existenz von Szenen und Milieus angewiesen, die einerseits kreative Prozesse anstoßen bzw. mittragen und andererseits als Konsumenten für die jeweiligen Produkte fungieren. Besonders augenfällig ist dies in Szenen der alternativen Musikproduktion, die sich von kommerzieller Massenproduktion scharf abgrenzen und ,ihre“ Kreativunternehmer unterstützen, indem sie deren Produkte durch den Besuch von Clubs und Festivals, den Kauf von Tonträgern auf alternativen Labels und im Internet usw. gezielt nachfragen. Diese Szenen und Milieus können zwar 
im Prinzip ausschließlich auf der Basis sozialer Beziehungen bestehen und ohne räumliche Kontexte auskommen - in der Praxis gibt es jedoch ausgesprochene „Raumanker“ in Form von Szenevierteln, zentralen Clubs, Veranstaltungsorten mit Symbolcharakter usw. Art und Prozeduren der Wertschöpfung - von der kreativen Idee, der Erfindung, der Erstellung eines handelbaren Produkts oder einer Dienstleistung bis hin zur Vermarktung des Produkts - sind vermutlich nicht nur an die jeweiligen Szenen bzw. Milieus (verstanden als Gemeinschaften mit besonders hoher Binnenkommunikation), sondern auch an deren Raumkonstrukte gebunden. In die Struktur einer Nischenökonomie sind daher Raumkonstrukte vermutlich bereits derart eingeschrieben, dass sie bei der Formulierung ökonomisch begründeter Interessen und Handlungsentwürfe seitens der Nischenakteure jeweils an zentraler Stelle zum Tragen kommen. Besonders deutlich wird dies an ökonomischen Arrangements, die an symbolische Orte wie Clubs, Galerien usw. gebunden sind.

2. Zur Frage der Divergenz bzw. Kongruenz von Raumkonstrukten in Governance-Prozessen: Stimmt die Vermutung, dass die kreativen Nischenökonomien auch von ihren Raumbezügen her als wertschöpfende „Verlängerung“ oder Konkretisierung milieubezogener „Produkt“-Ideen angelegt sind, so liegt es nahe, den Akteuren eine spezifische Perspektive auf städtische Kontexte zu unterstellen: Diese Kontexte stellen für sie dann sozialräumliche Folien dar, auf denen sie ihren Ideen jeweils einen bestimmten unverwechselbaren Ausdruck geben können. Die Folien können in ihrer jeweiligen Textur die Logik der gesamten Kulturproduktion beeinflussen. So waren z. B. Clubs in Abrissgebäuden phasenweise für die lokale Indie-Szene ${ }^{1}$ in Berlin unverzichtbarer ökonomischer und räumlicher Bestandteil der Musikproduktion. Wegen ihrer Integration in eine spezifische Produktionslogik (inklusive materieller Wertschöpfungsketten) waren diese Raumkonstrukte für die Szene-Akteure nicht verhandelbar oder willkürlich für externe Interessen veränderbar. Sie stellten somit eigenlogische Ausdifferenzierungen künstlerischer Ideen und ihrer materiellen wie auch symbolischen Repräsentationen in bestimmten Produkten dar. Eine Kommunikation mit politischen Akteuren, die in den Raumkonstrukten lediglich den Ausdruck einer marginalen Subkultur ohne größeren stadtkulturellen oder entwicklungspolitischen Wert sehen (so, wie dies noch in den 1990er Jahren oft der Fall war), ist unter diesen Umständen mit erheblichen Schwierigkeiten verbunden.

1 Produktions- und Konsumentenszene rund um unabhängige kleine Tonträgerfirmen (engl. independent label). 
Theoretische Ansätze, die die Spezifik der Entstehung von kreativen Nischenökonomien und ihrer Raumbezüge zu erklären und interpretieren helfen, wären überwiegend aus folgenden Basisbereichen zu beziehen:

- aus Milieuansätzen, die die soziale Konstruktion von Raum theoretisch als integralen Bestandteil der Formierung subkultureller Szenen und darin eingebetteter Kulturproduktion fassen können (Matthiesen 1998; Schulze 1994);

- aus Netzwerkansätzen, die die Verknüpfung der Akteure der Musikproduktion auf unterschiedlichen Handlungsebenen sowie deren lokale Verankerung in Szenen erklären (,,networks of musical creativity“, Leyshon 2001: 62);

- aus institutionentheoretischen Überlegungen, die die Formierung von Szenen, die soziale Bewertung kultureller Güter und die Routinisierung des Umgangs mit diesen Gütern zu erklären helfen; hier ist vor allem an Herleitungen aus Ansätzen gedacht, die die Entstehung informeller Institutionen thematisieren (aus der Sicht der Institutionenökonomik: North 1992; aus der Sicht des politischen Institutionalismus: Lauth/Liebert 1999);

- aus Governance-Ansätzen, die es erlauben, die Konfrontation von gruppenspezifischen Raumkonstrukten und zurechenbaren Interessen zu thematisieren (Mayntz 2004; Healey 2004). Hierbei wäre vor allem die Frage zu stellen, inwieweit bestehende Machtgefälle (z. B. aufgrund der Verfügbarkeit/Nicht-Verfügbarkeit materieller Ressourcen) partizipationsorientierte Aushandlungsprozesse erschweren und insbesondere die Handlungsspielräume der Nischen-Akteure einschränken.

\section{Steuerungsparadoxa und systematische Missverständnisse: empirische Fingerzeige}

Am Beispiel der Stadt Berlin kann nicht nur die politische Ambivalenz der Entstehung kreativökonomischer Nischen, sondern auch die soeben angesprochene Divergenz von Raumkonstrukten in der Kommunikation zwischen politischen Akteuren und Szene-Akteuren sehr klar aufgezeigt werden. Zunächst ist zu konstatieren, dass sich die Stadt, die von ihrer politischen Führung derzeit als heimliche Kulturhauptstadt Europas vermarktet wird, lange Zeit durch eine geringe politische Beachtung der kulturellen Nischen auszeichnete, die in den 1980er und 1990er Jahren entstanden waren. Obwohl Entwicklungen wie die Herausbildung einer europaweit einzigartigen lokalen Jazzszene, einer global tonangebenden Szene der elektronischen Popmusik sowie weiterer subkultureller „Kno- 
tenpunkte“ jeweils erhebliche Attraktivitätsfaktoren für Kulturschaffende, Unternehmen, Touristen und Kulturkonsumenten darstellten, war die politische Antwort auf derartige Entwicklungen bis zum Beginn des 3. Jahrtausends sehr zurückhaltend. Teilweise beruhte dies auf einem erheblichen Unwissen der Entscheidungsträger über die Binnenstrukturen und -prozesse der beteiligten Szenen, teilweise auch auf einer traditionell an der Förderung der etablierten Hochkultur ausgerichteten Kulturpolitik. Besonders im Rahmen der vom Berliner Senat seit Mitte der 1990er Jahre verfolgten rigiden Sparpolitik gingen die Anteile der Förderung so genannter Freier Gruppen (d. h. von kulturellen Akteuren auBerhalb der anerkannten Sparten des Theaters, der Oper, des Balletts und der Konzertmusik) an der Gesamtförderung stark zurück.

Anhand eines empirischen Streiflichts soll die Grundproblematik im Folgenden kurz erläutert werden, ohne den Anspruch auf Verallgemeinerungsfähigkeit der Befunde zu erheben. Vielmehr sollen divergierende Verständnisse kreativer Nischenproduktion durch Politik, Kulturschaffende und Intermediäre skizziert, Schnittstellen und Brüche der Kommunikation benannt und erste Thesen zur „Mechanik“ von Governanceprozessen rund um Nischenökonomien formuliert werden. Empirische Grundlage ist die Auswertung einer teilnehmenden Beobachtung einer Podiumsdiskussion zum Thema „Neue Musik und Jazz“ von kulturpolitisch engagierten Vertretern politischer Parteien, Musikproduzierenden in den Bereichen Jazz und Neue Musik sowie Intermediären (Veranstaltern, Initiativgruppen bzw. Netzwerken), die im Sommer 2008 in Berlin stattfand. Als Hauptproblem wurde von allen Beteiligten die mangelnde öffentliche Wahrnehmung der einzelnen Bereiche der nichtkommerziellen Musikproduktion herausgestellt. Sie wurde auf folgende Ursachen zurückgeführt:

1. An kreativen Nischenökonomien, speziell an der Hervorbringung improvisierter Musik, besteht kein größeres wirtschaftspolitisches Interesse. Förderer und Investoren sind kaum mit monetären Profitaussichten, sondern lediglich mit Imagegewinnen im Rahmen eines eher traditionellen Mäzenatentums zu gewinnen. Die öffentliche Förderung orientiert sich aufgrund der Durchsetzung der Prinzipien quasi-unternehmerischer Stadtpolitik an ähnlichen Kriterien. Dort, wo kein großes Publikum erreichbar ist und kommerzielle Gewinne zu erzielen sind, verspricht sich die Stadtpolitik auch nur wenig Imagegewinn. Auch die mangelnde Aussicht auf eine private Kofinanzierung von Projekten und Veranstaltungen hält die Stadtpolitik von einem Engagement in diesen Bereichen ab.

2. Von der Politik wird die Vernachlässigung „freier“ Szenen auch mit dem Argument verteidigt, es habe wenig Sinn, flüchtigen Szenerien 
und Subkulturen allzu viel Aufmerksamkeit zu schenken, weil man nie wisse, ob sich tragfähige Strukturen entwickelten und ob man auf der politischen Seite für ein förderndes Engagement auch tatsächlich etwas bekomme. Musiker und Intermediäre pochen dagegen auf kulturellen Minderheitenschutz und auf die Ausgleichsfunktion des Staates, der ein offensichtliches Marktversagen kompensieren solle.

3. Ebenfalls von der Politik kommt das - nicht ganz uneigennützige - Argument, dass mit einer staatlichen oder kommunalen Förderung subkultureller Akteure und ihrer Umgebungen genau das zerstört werde, was eigentlich das Wesen der kreativen Nischen ausmache: nämlich spontane Verlagerungen von Szenerien; die Entstehung passender sozialräumlicher Einbettungsformen in einzelnen Stadtquartieren, die meist im Windschatten ökonomischer Inwertsetzung liegen, niedrige Mieten und leerstehende Gebäude zur Zwischennutzung aufweisen usw. Für die Politik böten sich unter derartigen Bedingungen kaum Anhaltspunkte für fördernde Maßnahmen im Sinne eines soziokulturellen und zugleich städtebaulichen Bestandsschutzes.

4. Von den Musikproduzenten und Intermediären wird beklagt, dass die Zahl der Orte, auf die die Produktion improvisierter Musik angewiesen ist (z. B. für Proben, Aufführungen, Projekte, Musikertreffpunkte) in den vergangenen Jahren aufgrund ausgebliebener Förderung stark zurückgegangen ist. In diesem Zusammenhang werden Versuche der freien Musikszenen, Kommunikations- und Aufführungszentren zu schaffen, um stabile Anlaufpunkte für Musiker, Produzenten und Veranstalter zu bekommen, von der Politik kaum verstanden, in Einzelfällen in der Öffentlichkeit sogar ironisch kommentiert.

5. Von der Politik wird den „freien“ Akteuren ans Herz gelegt, sich zu Interessengruppen und Netzwerken zusammenzuschließen. Splittergruppen könnten von der Politik nicht Ernst genommen werden. Genau dies wird von den Betroffenen jedoch als Borniertheit der politisch Mächtigen und als Ignoranz gegenüber den schwierigen Produktionsbedingungen verstanden. Diese ließen für Lobbyarbeit oder politisches Engagement weder Zeit noch Energie übrig.

\section{Steuerungsperspektiven: zwischen direktiver Steuerung und Kontextsteuerung}

Am geschilderten Fall wird deutlich, dass Stadtpolitik und Governance gegenüber kreativökonomischen Nischen leicht einen Gestus der Ignoranz entwickeln können, der aus einer Mischung unterschiedlicher Dispositionen gebildet wird: von der Unkenntnis der ökonomischen und so- 
ziokulturellen Basisprozesse, von der Orientierung auf repräsentationstaugliche Großprojekte und -strukturen hin, vom Ziel der neo-liberalen Inwertsetzung vermeintlicher Aktivposten der „Hochkultur“ für eine „wettbewerbsfähige“ Stadtpolitik und von einer traditionellen Geringschätzung angeblich schwer zu konsumierender Minderheitengenres. Dies hat Konsequenzen für die Ausgestaltung der Interaktionsprozesse, aber auch für die Steuerungsperspektiven, die in Bezug auf diese Ökonomien entwickelt werden. Hierzu können zunächst nur vorläufige Thesen formuliert werden, die in späteren empirischen Untersuchungen auf ihre Gültigkeit hin überprüft werden müssen:

1. Es gibt zunächst keine „,natürlichen“ Anknüpfungspunkte zwischen der Politik und den Akteuren der kreativen Nischen. Kommunikationsgelegenheiten, die sich im Zuge der jeweiligen professionellen Tätigkeiten ergeben würden, sind rar. Interaktionen zwischen Politik, Verwaltung und Szene-Akteuren müssen eigens aus bestimmten Anlässen herbeigeführt werden.

2. Relevante Anlässe kommen in der Regel in zwei Handlungszusammenhängen zustande: 1 . in einem fest definierten formellen Rahmen, z. B. anlässlich der Bewerbung von Kulturschaffenden und Intermediären um staatliche bzw. kommunale Fördermittel, 2. im Kontext von lokalen Aushandlungsprozessen unter dem Eindruck von existentiellen Krisen, z. B. runden Tischen zur lokalen Kulturpolitik oder eigens initiierten öffentlichen Diskussionen selbstorganisierter Gruppen von Kulturschaffenden, die versuchen, als pressure groups tätig zu werden.

3. In Abhängigkeit von lokalen Kontexten und Diskurskulturen pendeln die Steuerungsperspektiven zwischen den Extremen „,gezielte Integration der Nischenökonomien in lokale Stadtentwicklung und globale Produktionsprozesse“ und „quasi-staatliche Planung für eine Vielzahl unterschiedlicher Interessen“. Dabei stellt sich heraus, dass das Interesse der Akteure der Nischenökonomien an lebendigen Szenerien, subkulturellen Gelegenheiten der Interaktion von Produzenten, Kulturschaffenden und Konsumenten sowie spontanen, ungeplanten Entwicklungen tendenziell mit den repräsentationslogischen und imageorientierten Interessen staatlicher und stadtpolitischer Akteure an der Formulierung marktkonformer, erfolgsorientierter Stadtpolitiken kollidiert. Zum einen sind die ökonomischen und sozialen Binnenstrukturen und -prozesse der Nischen nicht evident, d. h. sie müssen Außenstehenden erklärt und insbesondere im Hinblick auf ihre Fragilität und Prekarität begreiflich gemacht werden. Zum anderen sind politische Legitimierungsforderungen, die vom 
lokalen Staat an die Nischen gerichtet werden, von deren Akteuren nicht ohne weiteres zu erfüllen, da sich diese Forderungen meist an den Maßstäben orientieren, die an Mainstream-Ökonomien und ihre gut etablierten Institutionen angelegt werden.

Da die gegenwärtige Praxis der politischen Kulturförderung sich nach wie vor auf einer direktiven Steuerung mit Hilfe traditioneller Förderinstrumente gründet, wären neue Wege der Vermittlung zwischen den genannten Extremen erforderlich. Besonders unter den Rahmenbedingungen einer ökonomistischen Stadtpolitik stehen und fallen derartige Bemühungen mit den Aussichten auf die Einführung adäquater informeller und formeller Institutionen. Adäquat wären diejenigen Institutionen, die den kreativökonomischen Nischen eine Art Bestandsschutz sowie eine Entlastung von unmittelbaren ökonomischen Erfolgszwängen zusichern.

So wäre weniger die direktive Steuerung über die Förderung von Projekten als vielmehr die Unterstützung von einzelnen Akteuren und Netzwerken der kreativen Nischenökonomie unter Zusicherung der Entscheidungsautonomie über die Verwendung der Mittel zu erwägen. Auch die Pflege von ,zentralen“ Orten, die diesen Nischen als soziale Ankerpunkte dienen, müsste institutionell abgesichert und darüber hinaus öffentlich kommuniziert werden. All dies wäre somit eine Sache einer Kontextförderung, die die Rahmenbedingungen dafür schafft, dass sich kreativökonomische Nischen ziel- und ergebnisoffen entwickeln können. Dabei können konkrete Orte eine zentrale Rolle spielen - nicht nur für die Entwicklung interessanter Projekte und Produktionsstrukturen, sondern auch für die symbolische Selbstvergewisserung und das Selbstbewusstsein der kreativen Akteure. Auch dem Anspruch der auf die Reduzierung von Machtgefällen in der Kommunikation mit politischen Akteuren sowie in der Ausgestaltung konkreter GovernanceProzesse könnte eine Kontextförderung wesentlich entgegenkommen.

Inwieweit derartige Entwicklungen für beide Seiten attraktiv sein und von ihnen als erfolgsträchtig angesehen werden können, muss sich noch erweisen. Besonders der Antagonismus von kommerzieller Renditeorientierung und dem Anspruch auf kulturelle Autonomie erfordert Governance-Prozesse, die offene Aushandlungsprozesse, soziale Experimente und neue Wege der Selbstorganisation ermöglichen. Im Sinne der Prinzipien einer kommunikativen Stadtplanung (Healey 2002) wäre allein die öffentliche Diskussion über kreative Nischenökonomien bereits ein wichtiger Schritt zu ihrer Würdigung und partizipativen Aufwertung. Für die sozialwissenschaftliche Raumforschung entsteht dadurch ein neues Betätigungsfeld - es gilt künftig, die sozialen, ökonomischen und sozialräumlichen Kontexte der Ausdifferenzierung der Ni- 
schenökonomien behutsam zu erkunden sowie einschlägige Steuerungsprozesse auf ihre besondere Interaktionsdynamik hin zu analysieren.

\section{Literatur}

Bürkner, Hans-Joachim (2004): „Lokale Wissensmilieus und Kompetenznetzwerke mit heterogenen Ortsbezügen - Herausforderungen für eine irritierte Stadtpolitik“. In: Matthiesen, Ulf (Hg.), Stadtregion und Wissen. Analysen und Plädoyers für eine wissensbasierte Stadtpolitik, Wiesbaden: VS-Verlag für Sozialwissenschaften, S. 159169.

Cockburn, Cynthia (1977): The Local State: Management of Cities and People, London: Pluto Press.

Conell, John/Gibson, Chris (2002): Sound Tracks: Popular Music, Identity and Place. London, New York: Routledge.

Healey, Patsy (2002): „On Creating the ,City“ as a Collective Resource“. In: Urban Studies 39, S. 1777-1792

Healey, Patsy (2004): „Creativity and Urban Governance“. DISP 158, S. 11-20.

Krätke, Stefan (2002): Medienstadt. Urbane Cluster und globale Zentren der Kulturproduktion, Opladen: Leske und Budrich.

Lange, Bastian (2006): „Raumaneignungen und Vergemeinschaftungsformen von kulturellen Start-Up Unternehmern in Berlin“. In: Szope, Dominka (Hg.): Pragmatismus als Katalysator kulturellen Wandels. Erweiterungen der Handlungsmöglichkeiten durch liberale Utopien, Berlin: LIT-Verlag, S. 327-350.

Lange, Bastian (2007): Die Räume der Kreativszenen. Culturepreneurs und ihre Orte in Berlin, Bielefeld: transcript Verlag.

Lauth, Hans-Joachim/Liebert, Ulrike (1999): „Do Informal Institutions Matter? Informelle Institutionen in der interkulturell vergleichenden Partizipations- und Demokratisierungsforschung“. In: Lauth, HansJoachim/Liebert, Ulrike (Hg.), Im Schatten demokratischer Legitimität. Informelle Institutionen und politische Partizipation im interkulturellen Demokratievergleich, Opladen: Leske und Budrich, S. 1136.

Leyshon, Andrew (2001): „Time-space (and digital) compression: software formats, musical networks, and the reorganisation of the music industry“. In: Environment and Planning A, 33, S. 49-77.

Mager, Christoph (2007): HipHop, Musik und die Artikulation von Geographie. Stuttgart: Franz Steiner Verlag (Sozialgeographische Bibliothek, 8). 
Matthiesen, Ulf (1998): Milieus in Transformationen. Positionen und Anschlüsse. In: Matthiesen, Ulf (Hg.), Die Räume der Milieus, Berlin: Edition Sigma, S. 17-79.

Mayntz, Renate (2004): Governance Theory als fortentwickelte Steuerungstheorie? Max-Planck-Institut für Gesellschaftsforschung, Working Paper 04/1 (www.mpi-fg-koeln.mpg.de, Zugriff am 19.7.05).

North, Douglass C. (1992): Institutionen, institutioneller Wandel und Wirtschaftsleistung. Tübingen: Mohr (Die Einheit der Gesellschaftswissenschaften, 76).

Scharenberg, Albert/Bader, Ingo (2005): Der Sound der Stadt: Musikindustrie und Subkultur in Berlin, Münster: Westfälisches Dampfboot.

Schulze, Gerhard (1994): „Milieu und Raum“. In: Noller, Peter/Prigge, Walther/Ronneberger, Karl (Hg.), Stadt-Welt. Über die Globalisierung städtischer Milieus, Frankfurt/Main: Campus Verlag, S. 41-53. 



\section{Kultur und Kreativität als Indikatoren des stadtentwicklungspolitischen Zeitgeistes? \\ Vom Wandel der Entwicklungsstrategien \\ der Internationalen Bauausstellung \\ IBA Emscher Park und IBA Hamburg}

\section{CONSTANZE GÜNTHER, ACHIM PROSSEK}

Im Instrument der Internationalen Bauausstellungen (IBA) bündeln sich zeitgemäße Fragen und Tendenzen der Stadt- und Regionalentwicklung. Denn im Verlauf ihrer hundertjährigen Genese avancierten diese von Bauausstellungen im engeren Sinne zu Strukturprogrammen mit einem breiten Themenspektrum, die sich in einem begrenzten Zeitrahmen heute mit komplexen Entwicklungsaufgaben konfrontiert sehen. Neben originär städtebaulichen Aufgaben wie dem Umbau und der Neugestaltung von Quartieren und (Stadt-)Landschaften, werden IBAs spätestens seit den 1980er Jahren auch zur Positionierung von Städten und Regionen im globalen Wettbewerb um Menschen und Investitionen ins Leben gerufen. Dieser Prozess ist verknüpft mit der Herstellung städtischer und regionaler Identität. Ein Ziel, das sich vermehrt in einem strategischen Einsatz von Kunst, Kultur sowie neuerdings kreativwirtschaftlichen Handlungsanleitungen für die Revitalisierung urbaner Räume äußert. Vor dem Hintergrund der planungspolitischen Kontextualisierung von Bauausstellungen, die seit der Nachkriegszeit allesamt kommunal oder staatlich initiiert waren, avancieren gerade die jüngeren IBAs zu einem interessanten Untersuchungsgegenstand für das Themenfeld der Urban Governance. Das allen „kreativen“ Strukturprogrammen inhärente Steuerungsparadoxon wird dabei von einem zweiten Paradoxon begleitet, welches das Format IBA selbst betrifft: Die „Exterritorialität“ von 
Bauausstellungen, die durch befristete Sonderkonditionen finanzieller, politischer und administrativer Art und das Credo eines Experimentierraums auf Zeit konstruiert wird (M:AI 2007: 22), divergiert mit dem Erfolgsdruck von inhaltlichen und zeitlichen Zielvorgaben. In der Praxis treffen der Realisierungsdruck von zeitlich terminierten, entwicklungspolitischen Vorgaben, wie etwa das Hamburger Leitprojekt „Sprung über die Elbe“" und seine Erfüllung durch die IBA Hamburg, auf das prozesshafte und situative, ergebnisoffene Selbstverständnis einer IBA. Ein vergleichbarer Konflikt im Ruhrgebiet resultierte aus der klassisch wirtschaftspolitischen Forderung von Land und Kommunen an die IBA, möglichst viele Arbeitsplätze zu erschaffen, wozu diese qua Entwicklungsansatz nur bedingt in der Lage war.

Die Gegenüberstellung der IBA Emscher Park (1989-1999) mit der IBA Hamburg (2007-2013) verdeutlicht den Wandel, den Urban Governance-Methoden in den letzten zehn Jahren im Umgang mit Kunst und Kultur erfahren haben. Während im Ruhrgebiet Kunst und Kultur als strategische Instrumente für die Landschaftsentwicklung und die regionale Bildproduktion eingesetzt wurden, die sich vor allem in ihrer Ausprägung als Industriekultur niederschlug, rücken bei der IBA Hamburg ganzheitliche, kreative Revitalisierungsstrategien über die Stärkung und Implementierung von kreativen Ökonomie-Clustern stärker in den Mittelpunkt.

Die IBA Emscher Park wurde aus der Einsicht gegründet, dass bisherige Strategien zur Bewältigung des Strukturwandels nicht den gewünschten und benötigten Erfolg brachten. Investitionen in den Infrastrukturausbau und in klassische Wirtschaftsförderung hatten seit Ende der 1960er Jahre die Strukturprobleme des Ruhrgebiets nicht entscheidend lindern können. Weiche Standortfaktoren gewannen an Bedeutung, ökologischer Stadtumbau wurde zu einem Leitmotiv. Programmatisch wurde die IBA Emscher Park „Werkstatt für den Umbau alter Industrieregionen" genannt. Kreativ war, das Instrument Bauausstellung zu regionalisieren, eine alte Industrieregion ökologisch und nachhaltig umzubauen und die benötigten Gelder aus den verschiedensten Fördertöpfen zusammenzuführen, Projekte mit ,instrumentellem Erfindungsreichtum“ zu realisieren, wie Direktor Karl Ganser es einmal formulierte. Er sah darin eine der von außen kaum wahrnehmbaren Leistungen der IBA.

Diese Leistung wurde überwiegend in einem kleinen Kreis und mit ausgewählten Personen realisiert. Die über 400 Ideen aus dem ursprünglichen Projektaufruf blieben unberücksichtigt, was der IBA den Vorwurf von elitärem Handeln einbrachte, sie aber konsequent ihre Vorstellungen im Rahmen von sieben Leitprojekten umsetzen ließ, welche mit unterschiedlichem Erfolg durchgeführt wurden: Emscher Landschaftspark, 
Ökologischer Umbau des Emscher-Systems, Erlebnisraum Rhein-HerneKanal, Industriedenkmäler als Kulturträger, Arbeiten im Park, neue Wohnformen und Wohnungen sowie neue Angebote für soziale, kulturelle und sportliche Tätigkeiten. Letzteres Themenfeld fokussierte ausdrücklich nicht den Bereich der Erwerbsarbeit, sondern nahm die in der Region zahlreich vorhandenen Personen ohne Erwerbstätigkeit, Arbeitslose und Rentner, in den Blick. Auch „Arbeiten im Park“ war nicht auf eine bestimmte Berufsgruppe bezogen: Architektonisch und landschaftsplanerisch hochwertige Gewerbegebiete und Gebäude sollten hochwertige Arbeit anziehen. Das ist bis heute nur teilweise gelungen.

Die IBA Hamburg ist ein Ergebnis und zugleich Instrument des struktur- und stadtentwicklungspolitischen Gesamtkonzeptes „Metropole Hamburg - Wachsende Stadt“. Die Fokussierung auf den Entwicklungsraum des Hamburger Südens, der seit der Flut 1962 stark vernachlässigte wurde, u. a. ablesbar an dem bis weit in die 1970er Jahre anhaltenden Investitionsstillstand im Stadtteil Wilhelmsburg, nimmt ihren Anfang in den Planungen für die „Hafencity Hamburg“. Die mit diesem Großprojekt seit den 1990er Jahren eingeleitete Öffnung der Stadtmitte zur südlichen Seite der Elbe markiert den Ausgangspunkt für eine Entwicklungsstrategie, die mit der Bewerbung um die Ausrichtung der Olympischen Spiele auf einer innerstädtischen Konversionsfläche am gegenüberliegenden Elbufer fortgesetzt wurde. Zwar scheiterte die Olympiabewerbung, stieß jedoch eine Metropolisierungspolitik an, die 2002 in der Verabschiedung des Hamburger Leitbildes „Metropole Hamburg - Wachsende Stadt" resultierte. Die großflächig angelegte Wachstumsstrategie soll mithilfe von fünf Leitprojekten, zu dem auch der „Sprung über die Elbe“ gehört, u. a. das bislang vernachlässigte stadtentwicklungspolitische Potenzial des Hamburger Südens erfassen.

Eines der entscheidenden Instrumente zur Umsetzung dieser Zielvorgabe ist die IBA Hamburg. In dem verhältnismäßig kurzen Realisierungszeitraum von 2007 bis 2013 kommt ihr die komplexe Aufgabe zu, die Elbinseln Wilhelmsburg, Veddel und den Harburger Binnenhafen von ihrem historisch gewachsenen Stigma zu befreien und mit gezielten Entwicklungsprogrammen in das Gesamtgefüge Hamburgs einzubinden, um sie damit auch für neue Bevölkerungsgruppen langfristig interessant zu machen. Mit der 2013 auf der Elbinsel parallel stattfindenden Internationalen Gartenschau wird diese Zielsetzung zusätzlich auf landschaftsplanerischer Ebene unterstützt.

Die Themen der IBA Hamburg wurden aus dem Ort heraus entwickelt. Die Flussinsel Wilhelmsburg ist deutlich von einer funktionellen, aber auch sozialen Verinselung geprägt: Auf der physischen Ebene äußert sich diese in einem parzellierten, von Verkehrsschneiden geformten 
Nebeneinander aus Hafen und Industrie, Wohngebieten, Landwirtschaft und Brachflächen und nicht zuletzt in einer durch die Insellage bedingte permanente Hochwassergefahr, auf der gesellschaftlichen Ebene in einer räumlichen Separierung verschiedenster sozialer Milieus. Wilhelmsburg und die Veddel sind mit ca. 50.000 Einwohnern aus mehr als 40 Herkunftsländern dabei nicht nur die jüngsten Stadtteile in Hamburg, sondern auch jene, die einen überproportional hohen Anteil an Hartz IVEmpfängern und Langzeitarbeitslosen aufweisen.

Mit ihrer Aufgabenstellung „Entwürfe für die Metropole von morgen“" greift die IBA Hamburg drei essentielle, stadtentwicklungs-, aber auch bildungs- und kulturpolitisch relevante Themen ihres Austragungsortes auf: Die Frage nach dem für Deutschland noch immer konfliktreichen Selbstverständnis im Umgang mit einer internationalen Stadtgesellschaft (,Kosmopolis“), die Gestaltung von Grenz- und Übergangsorten einer Metropole („Metrozonen“) sowie den Umgang von Städten mit dem Klimawandel.

\section{Der globale Standortwettbewerb - ein ,creative overkill“?}

Fast scheint es so, als stehe Kreativität doch in nahezu allen, zumindest überregional agierenden Städten weit oben auf der Stadtentwicklungsagenda - als Standortfaktor und Beschäftigungsmotor, aber auch als Imagefaktor und Ausdruck eines neuen „Placemakings“. Der vielerorts eventisierte und ökonomisierte Kultureinsatz steht sinnbildhaft für den Wunsch nach einer Repräsentierbarkeit, die zugleich eine Planbarkeit suggeriert, die mit spezifischen Governance-Methoden erreichbar scheint. Kulturelle und kreative Revitalisierungsstrategien zeichnen sich dabei vorwiegend durch Leuchtturmprojekte und Cluster-Strategien aus. Die Stadtentwicklungsinstrumente IBA Emscher Park und IBA Hamburg verdeutlichen nicht nur den Steuerungsbedarf und -willen von Städten für dieses Themenfeld, sondern auch die Weiterentwicklung des Diskurses von Kunst und Kultur als weichen Standortfaktoren in den 1990er Jahren hin zu ganzheitlichen kreativen Revitalisierungsstrategien im 21. Jahrhundert.

Die industriekulturellen Objekte und die Route der Landmarkenkunst sind sicherlich die bedeutendsten Ergebnisse der kulturellen Entwicklungstrategie der IBA Emscher Park im Ruhrgebiet. „Kulturell“ heißt hier mehreres: mit historischem Bewusstsein auf regionale Eigenheit und Identität zielen, ebenso wie Orte für Kultur zu schaffen, etwa auf den Zechen Zollverein in Essen oder Consolidation in Gelsenkir- 
chen. Die IBA selbst hatte mit dem Festival „Musik im Industrieraum“ für eine Hallennutzung gesorgt, mit einem Konzept, welches seine Herkunft und Lokalität stets mitthematisierte, ohne rein ortsspezifische Kunst zu verkörpern.

Auch um die dauerhafte Bespielung zu gewährleisten, hat das Land Nordrhein-Westfalen 2001 die Ruhrtriennale gegründet, die seitdem hauptsächlich industriekulturelle Orte für ihre Aufführungen nutzt, allen voran die Bochumer Jahrhunderthalle als aufwendig umgebautes und modernisiertes Flagschiff. Und die Objekte der Landmarkenkunst reflektieren den Raum, in dem sie stehen. Richard Serras Bramme für das Ruhrgebiet, der Tetraeder von Wolfgang Christ und Jürgen LIT Fischer, Hermann Priganns Himmelstreppe oder Mischa Kuballs Yellow Marker und alle anderen Landmarken ordnen und strukturieren den Raum, lassen ihn als zusammengehörig erscheinen.

Die Intention war, eine Vorstellung, ein Bild von der Region herzustellen, weil in seinem Fehlen sowohl ein Orientierungs- als auch ein Identifikationshindernis gesehen wurde. Beide Defizite wurden verringert. Gegen Ende der IBA setzte die Sorge um die Vermittlung der eigenen Leistung ein. Man hielt die Probleme für zu komplex und den Raum insgesamt für zu groß, um die IBA vollständig kommunizieren zu können. Weil die Binnenbekanntheit hoch war, ging es im Abschlussjahr nur noch um die Außenwirkung: Vier große Ausstellungen wurden kommuniziert, mit der Installation The Wall von Christo \& Jeanne Claude im Gasometer Oberhausen als eigens inszeniertem Höhepunkt. Die Strategie der Kommunikation kultureller Großereignisse folgte der Marketinglogik begrenzter Aufmerksamkeitsökonomie, führte aber zu dem Vorwurf von Festivalisierung und Abgehobenheit.

Dass diese Ausstellungen für die IBA nur als Medium gedacht waren, über die die Wahrnehmung auf die Gesamtleistung gelenkt werden sollte, ging weitgehend unter - und die Strategie so auch nicht auf: Viele Objekte und Themen konnten nie die Aufmerksamkeit erzielen, die der Industriekultur zuteil wurde. Beispielhaft dafür ist das Themenfeld Wohnen: Weder die ökologische Gestaltung noch die innovative Planung noch die großen Siedlungen selbst wie die CEAG-Bebauung in Dortmund sind einer breiteren Öffentlichkeit bekannt.

Was bleibt ist also die Frage nach der langfristigen Strukturwirksamkeit beispielhafter Projekte. Die IBA Hamburg verfolgt mit ihrem Projekt „Kreatives Quartier Elbinsel“ eine Zielvorgabe, die das Steuerungsparadoxon des Kreativitätsdiskurses bereits im Titel trägt. Im Rückblick auf die Genese des Projekts tritt dabei zunächst ein prozesshaftes Verständnis im Umgang mit dem kulturellen und kreativen Feld zutage, ablesbar an der Neukonzeptionierung der Kunst- und Kulturför- 
deraktivitäten der IBA Hamburg: Während sich das Auftaktjahr 2007 noch als eventisierte Großveranstaltung mit mehr als 60 Einzelprojekten zeigte, präsentierte die IBA Hamburg Anfang des folgenden Jahres eine überarbeitete Konzeption, die mit fünf Kontext- und Infrastrukturfördermaßnahmen nunmehr die Initiierung einer „schrittweisen kreativen Quartiersentwicklung“ einleiten soll.

Mit der Infrastrukturfördermaßnahme Räume für die Kunst erhofft sich die IBA Hamburg eine nachhaltige Verankerung der künstlerischen und kreativen Szene vor Ort. Dabei reagiert sie auf den jetzt schon geringen Leerstand in Wilhelmsburg mit der Bereitstellung von mietgünstigen Atelier-, Ausstellungs- und Produktionsflächen. Als modellhaftes Projekt soll die Immobilie „Veringhöfe“ zu einem solchen Ort entwickelt werden - basierend auf einer Vorgehensweise, die die künftigen Nutzer von Beginn an in den Entwicklungsprozess einbindet. Die Immobilie befindet sich dafür an einem durchaus günstigen Standort: das Reiherstiegviertel in Wilhelmsburg ist eines der attraktivsten Quartiere der Elbinsel, mit einer anziehenden gründerzeitlichen Bausubstanz, einer durch die städtische Wohnungsgesellschaft SAGA GWG subventionierten, stetig wachsenden studentischen Klientel und einer kleinteiligen Dienstleistungsinfrastruktur. Reaktionen auf den ersten, im November 2008 abgehaltenen offenen Workshop zur Immobilie zeigen, dass das Projekt zwischen einer positiven Akzeptanz durch die künstlerische Szene einerseits und dem Vorwurf einer zielgerichteten neoliberalen Stadtentwicklungspolitik andererseits balanciert. Eine Stabilisierung der Räumlichkeit und eine langfristige Absicherung für die potentiellen Mieter auch über 2013 hinaus, möchte die IBA Hamburg mithilfe eines neuartigen Nutzer- und Betreiberkonzept lösen, das bislang jedoch noch undefiniert ist.

Das Handlungsfeld der kreativen Ökonomien unterliegt einer interessanten Entwicklung. Nach Befürchtungen der ersten kritischen Stimmen, das Thema bediene die übliche Methodik der Ansiedlung kreativer, bereits etablierter Akteure am „Pioniersstandort Wilhelmsburg“, zeichnet sich derzeit eine Entwicklung von Seiten der IBA ab, die das Thema mit einer endogenen Strategie neu zu justieren versucht. Unter dem Label „Kunst macht Arbeit“ möchte die IBA Hamburg geläufige kreativwirtschaftliche Ansätze auf Stadtteilebene übersetzen und mit dem Beschäftigungssektor zusammenbringen. Aktuell formieren sich in Wilhelmsburg dazu zunächst einmal vielversprechende Kooperationsprojekte zwischen Künstlern und Kreativen, Qualifizierungsträgern und lokalen Initiativen, die von IBA-Seite von Beginn an mit den relevanten behördlichen Vertretern zusammengebracht werden. Die IBA Hamburg zeigt 
sich in diesem Kontext also stärker als katalysatorische und vernetzende Institution, denn als originäre Projektentwicklerin.

Weitere Maßnahmen beinhalten ein unabhängiges, kuratiertes Format für ortsspezifische Kunst im urbanen Raum - den seit 2008 jährlich stattfindenden Elbinsel Sommer -, der Raum für die (immaterielle) künstlerische Reflektion der IBA-Themen bieten soll; die Förderungen von temporären Großevents wie ortsspezifischen Festivals, aber auch einer aus dem Stadtteil stammenden Veranstaltungskultur; sowie einer permanenten theoretischen Reflektion, die Künstler in einen Dialog mit Stadtplanern bringen soll. Der Titel der letzten IBA-Publikation „Kunst und Stadtentwicklung. Das Betreiben eines vegetarischen Restaurants mit einer Horde Kannibalen“" bringt die Ambivalenz der beschriebenen Steuerungsproblematik dabei durchaus humorvoll auf den Punkt. Inwiefern die beschriebenen Maßnahmen tatsächlich zu einer „kreativen Quartiersentwicklung“ beitragen, bleibt zum jetzigen Zeitpunkt offen, da aufgrund der frühen Projektphase noch keine projektübergreifende Analyse geleistet werden kann.

\section{Möglichkeiten der Intervention aus stadtentwicklungspolitischer Sicht}

Die Gegenüberstellung der IBAs Emscher Park und Hamburg und ihrer Themen Industriekultur und kreative Quartiersentwicklung veranschaulicht den neuen Steuerungsbedarf von Städten und Regionen im Kontext der Transformation von der Industrie- zur Wissensgesellschaft. Der Vergleich der in den IBA-Projekten auftretenden Governance-Methoden zeugt dabei nicht nur von einer inhaltlichen Begriffsverschiebung von Kunst und Kultur zu Kreativität, sondern verdeutlicht eine substantielle Erweiterung des Themenfelds: von der Konsumtions- zur Produktionsebene und von der reinen Bildproduktion zur Beschäftigungspolitik. Konnte die IBA Emscher Park letztlich nur hoffen, dass in ihren anspruchsvollen Bürogebäuden (etwa dem preisgekrönten Wissenschaftspark Rheinelbe in Gelsenkirchen) entsprechend höherwertige Arbeitsplätze angeboten werden, begleitet die IBA Hamburg den Prozess über die Bereitstellung der Räume hinaus.

Dabei gilt umso mehr: Ein praxisorientierter Umgang mit dem Steuerungsparadoxon der Kreativität artikuliert sich in GovernanceStrategien, die auf die Mechanismen einer ,geplanten Ungeplantheit“ setzen. So lässt sich am Beispiel der 1BA Hamburg eine Mischung aus exogenen und endogenen, Top-down- und Bottom-up-Strategien ablesen, die dem globalen Streben nach Kreativitätspolitiken durchaus ent- 
sprechen, den Steuerungsanspruch durch ihre standortspezifische, auf die Quartiersebene zugespitzte Ausrichtung aber gleichzeitig zu relativieren versuchen. Dem Versuch der räumlichen Verortung von kreativen Akteuren, die sich aber u. a. durch nur schwer räumlich fassbare Attribute wie Mobilität und Flexibilität auszeichnen, begegnet die IBA Hamburg mit der Schaffung von offenen Möglichkeitsräumen: Indem Künstler und kreative Akteure frühzeitig in konkrete Planungen für potentielle Themenimmobilien eingebunden werden, bleibt der Ort zwar nach wie vor strategisch gesetzt, die räumliche und konzeptionelle Ausgestaltung sowie die „kreative Aneignung“ desselben jedoch der Eigenverantwortlichkeit der Akteure überlassen. Mit diesem Vorgehen erhofft sich die IBA Hamburg eine längerfristige und damit nicht ohne weiteres austauschbare Bindung der kreativen Akteure an den Standort. Erste Erkenntnisse über die Wirksamkeit der beschriebenen Strategien dürften bis zum Zwischenpräsentationsjahr 2010 vorliegen.

Die Weiterentwicklung des Diskurses im Rahmen von Bauausstellungen besteht also in der Möglichkeit, unkonventionelle und neuartige Herangehensweisen mit dem heute um den Faktor Kreativität erweiterten Themenfeld zu erproben. Der Status von IBAs als „Laboratorien auf Zeit" bietet ihnen die - wenn auch bisher nur zaghaft ergriffene - Chance, den Spagat zwischen einer eventisierten Aufmerksamkeitspolitik einerseits und einer Infrastruktur-, Kontext- und Vernetzungspolitik andererseits zu gestalten, die für sich beansprucht, Kunst, Kultur und Kreativität als Ressourcen und Katalysatoren ,nachhaltiger“ und produktiver als früher einzusetzen.

\section{Literatur}

M:AI (2007) (Hg.): IBA meets IBA. Eine Ausstellung zur hundertjährigen Geschichte der Internationalen Bauausstellungen. Essen. 
The Future 



\section{The Challenge of Governance in the Creative and Cultural Industries ${ }^{1}$}

\section{ANDY C. PRATT \\ Introduction}

The aim of this paper is to provide a survey of the cultural economy, that is the relationship between culture and the economy. We often think of culture and economy as separate domains, each not to be polluted by the other. In practice, I will argue, the two are inextricably linked through practice. Moreover, we commonly think of economy and culture as fixed in some way, however, once again our experience is that they are endlessly mutable. My theme is that culture, and particularly cultural production - the making of culture - has been transformed in recent years (it is always being transformed, but I only want to focus on the last 50 years, and it is this period that does seem to have moved cultural production onto a new level).

If we accept these points of view, then I think we must also accept another consequence, or rather a challenge: that of re-thinking our relationship to culture; in particular what I want to argue is that our collective relationship to culture - that is the domain of public policy - needs to change even if we are to engage with culture in the same ways as before, let alone exploring new ways. Business as usual is not an option. Of course culture is a broad canvas, I want to focus my attention of that area referred to as the cultural and creative industries - I'll clarify what I mean by the cultural and creative industries below. So, in brief, culture

1 An earlier version of this paper was given at the 50th anniversary lecture of the Gulbenkian Foundation. 
is changing, and culture's 'tentacles' seemingly embrace all areas of our lives today. Aside from an expanded and transformed cultural domain, we can point to the economic dimensions of cultural production - here we can point to the power of the film industry and Hollywood (Wasko 2003); however, we can point to the 'new kid on the block' - the computer games industry - which is bigger in economic terms than the film industry, worth 7 billion dollars a year in sales, and growing at $15 \%$ per annum (Crandall/Sidak 2006).

This revealed massive economic role of the computer games industry is a rebuff to those who dismiss the cultural economy as mere entertainment, 'candyfloss', or in other ways not part of the 'real economy'. The computer games industry is merely the visible part of the iceberg that is the cultural economy. As I will point out below, the cultural economy is bigger in scale that many traditional elements of 'the economy'. This fact matters for at least three reasons. First, the cultural economy is becoming a driving force in many urban and regional economies, in London it is the third largest sector of employment (GLA Economics, 2002).

Second, national and local governments have traditionally sought to govern major motors of their economies to facilitate growth. No such role is played in relation to the cultural and creative economy, leaving economies open to considerable risk and the potential of losing the benefits of vibrant cultural economies.

Third, underpinning both points is the fact that we lack an evidence base and sound understanding of the cultural economy. We can illustrate this by returning to the case of the computer game industry. The industry is hardly twenty years old, up to five years ago it was not even classified as an industry in the US Business Census (nor in Europe). In effect, the industry did not officially exist, and politicians and policy makers felt comfortable ignoring it, yet in fact it was a major driver or regional and national economies. This is not a mistake that we can afford to make in relation to the rest of the cultural sector.

Culture also finds its way into social and community policy: we have all heard about art being used to rehabilitate the excluded, or as a means of social engagement - and, it does work; even businesses recognise the role of culture in team building. Traditionally culture has come onto the state agenda in the guise of censorship, or as it is more politely called these days, regulation. However, with the rise of the economic power of the media for example there are also debates about monopolies and competition policy. The relationship between the regulation and competition policy is a difficult one. Finally, in this brief list, I would like to point to one area that I am particularly concerned with that is the 
spatiality of culture. In particular, the location of cultural facilities and production has consequences for access and participation, as well as the sometimes-considerable economic benefits of either Hollywood, or Bilbao.

The conceptual lens that I want to use to help us navigate these shifts is threefold: we need to examine the concept of culture, the making of culture, and the governance of culture; individually and in relation to one another. In effect, we need to find a new settlement or balance between them. Alongside this analysis I want us to keep in mind two other tensions. First, the tension of production and consumption - whilst we traditionally seem these as a dualism, I want to encourage us to think of them as a co-construction, a duality. Second, as I have already mentioned I want to suggest a similar perspective on culture and economy. In particular I want to flag up the subtle differences between the 'adjectival' cultural economy, and the 'noun' cultural economy. The former is the 'culturalisation thesis' where we argue that everything has become cultural (or the obverse, everything has become economic). The second, which I feel is more useful, is the focus on those activities that constitute cultural production (and this consumption, distribution, etc.). More of this later.

\section{Traditional Formations}

In this section I want to introduce the notion of the Concept-MakingGoverning in the cultural sector as applied to the empirical conditions in Europe in the mid-late 20th century. My purpose in choosing this schema is to resist the dualisms of public-private, state-market, high-low culture, culture-not culture. Which, whilst they do have empirical validity, are rooted in particular times and places, and hence less helpful for trans-historical and international work. I will try to challenge the usual dualistic conceptions of culture with a 3-way tension.

\section{Concept}

It is important to know what we are debating, and what assumptions we bring with us. We begin with the easy question, what is culture? Whilst there is a small library devoted to this topic and the very word is multiply contested I think that one way of cutting into this Gordian knot is via the idealist, naturalistic and sacred. This is a particularly dominant social formation of culture, one that has been influential in policy debates, it characterises culture as removed from the everyday, mundane and functional and elevates it to its own level - for some almost equivalent to a 
deity. Culture is 'over there' as some critics have put it this is a 'superorganic' conception; one that exists, beyond us, and one that if suitably prepared we may access in awe and wonder.

More specifically, there is considerable debate about the last point, our relation to culture (and it is important to this paper in the sense that we want to think about how culture is produced, or re-produced). Writers from the Frankfurt school, especially Adorno (1991), created a powerful argument about the relation that we might have to art and culture, by mobilising the notion of the 'aura' of a piece of art. Adorno is of course the progenitor of the term the 'culture industry' that he so distained (because of its mass production and loss of aura).

Here we can see the roots of the rejection of mass production, the opposition of the economic and the aesthetic that have come to codify the European and an international tradition in culture. I just want to raise the question of how aesthetic values are created, or where the hierarchies come from. They may be naturalised, and/or coincide with institutional power and authority (commonly academics/curators). Second, I want to point to the implicit notion of absolute values of art and culture (like the economic, but in a separate realm).

This point deserves a whole chapter to itself; however, we must be content with a few sketches here. At first sight - and in normative analyses - the 'object' of cultural policy is very difficult to define. It has two dimensions. First, the identification or definition of cultural value; second, the translation of that value into common terms/language that facilitates comparison across a variety of cultural (and non-cultural) forms and activities. Traditionally, such a mechanism facilitates allocation of resources. My point here is that the normative approach rests upon a third problem: the assumption that cultural value is absolute, unchangeable and universal. There is a body of research on this, perhaps best represented by Bourdieu's $(1979,1993)$ work. In summary he points to the recursive social construction of the field and the object (or, art and the audience, or again, production and consumption). Bourdieu argues that cultural value is created, and maintained, by - in his view - the education system. Regardless of whether ones accepts or rejects Bourdieu's analysis his approach offers a fatal blow to the tenet that there are universal or stable values to art. Logically, it follows that the problem of governance is catapulted from a technocratic issue off stage, to central stage in the political realm and policy making. I argue that this should lead us to explore a democratic modality for planning and policy legitimation. Moreover, that there is ample role, even a significant need, for the 'creation' or shaping of demand as there is in supply. An alternative way to characterise culture draws more upon a materialist frame of ref- 
erence, that stresses the anthropological making and re-making of culture; culture as ordinary, and in effect culture as profane rather than sacred. It is this source that I think is more helpful in guiding current discussions. However, the point I want to stress is that (and I have simplified it a lot) when we argue about culture we adopt a number of different points of departure, and as often as not we do not argue over the same things; or, the policy and the concepts are out of sync.

\section{Making}

Within the idealistic tradition of thinking about culture, one that I would argue has been dominant (although not universal) in the mid/late 20th century, the conception of what an artist, or a 'maker' is, is shaped in a particular manner.

The classic mid-20th century model is of the artist as individual genius often located in isolation from society who produces great art. Whilst artists have natural talents they have to be honed, through training, and then internalised as demonstrated by discipline and a technique. The artist has a sensibility that is trained through a knowledge and familiarity with the artistic canon. Such a conception focuses upon the support of the artist, so that they can lead an ascetic life free from pressures of money; hence the common state grant system. Later, state funded training, and support for exhibition/performance. So, we have the construction of an artist, and in part a governance system. Such a governance system became fully fledged when coupled with the discipline of economics.

\section{Governing}

It follows from the 'super-organic' conception of arts and culture that it is a 'good thing'; many critics have extended the notion to an essential component of humanity (especially when coupled with notions of civilising restraint), or to various ideas of transcendence. We can note that in the development of the modern state, education takes on a central role; it is no surprise that much of the growth of, for example, museums and galleries owe their existence to an educational agenda. However, there is not space to explore these debates, or those associated with nationalism and colonialism here.

I want to point to one critical technology of government that has shaped these broader concerns, and delivered a mechanism and rationale for cultural funding beyond mere pleading for a protection of 'the good life'. The rationale provided by welfare economics is that of public 
goods and market failure. I do not want to go into detail about this here, suffice to say that the result of market forces is an under-provision of certain types of cultural goods; thus, the state has to step in to provide them to maintain the public good. Added to this is a more precise argument specific to the arts referred to as Baumol's (Baumol/Bowen 1966) cost disease. This states that a performance of a string quartet cannot achieve efficiency gains of time saving, or labour saving, however, labour costs rise; thus live music becomes more and more uneconomic, and thus needs subsidy. If we add the notion of the corruption of culture by the masses, and cultural elitism as well as the attempt to develop cultural hegemony to reinforce nation building we have a strong combination.

Such an argument underpins what we might recognise as cultural policy in the mid-20th century. However, it also has its own inherent weaknesses in dealing with changes in cultural expression and form. Moreover, being linked to state budgets and therefore in competition with other services like, the military, or health, culture tends to lose out. As we will note, state culture budgets are subject to variation and uncertainty, which makes cultural sustainability precarious. Added to this is a politicised and idiosyncratic definition of which cultural forms and practices 'deserve' support. We can note that by definition the arts and culture are separate from commercial culture (despite evidence to the contrary). Finally, we can note that such a formation tends to a very staid, or conservative, view of culture that is backward looking, reverential and not dynamic.

The point of the remainder of this paper is that whilst such a formation of concept-making-governing created a consensus. Language and technology of governance (that is the relationship between the three components), it must necessarily be situated within a particular formation of economy, culture and the state. If, as I will argue, these three forces are themselves being transformed then the cultural formation is destabilised. In the next section I want to illustrate some of the forces of destabilisation; then I will follow with a 'new settlement' of conceptmaking-governance that is underpinning current trends in cultural policy. Finally, I will discuss the further challenges that we face in this field. 


\section{The Forces of Change}

In this section I want to outline some of the forces of change that have upset the old settlement; my point is that the change is not simply a matter of degree, but that it is transformative. As such 'business as usual' in the policy field is not possible (even if we only seek to preserve the status quo). I want to look at three forces (economy, culture, state); of course, these are not autonomous from one another or from society as a whole. However, for the purposes of argument we can focus on these manifestations of forces.

\section{Economy}

It is a familiar story to us all that the economic foundations of societies are shifting - they always do. In particular I want to focus on the decline of manufacturing industry in Europe, its migration to the global South; and the concentration of the service sector in the global North, moreover, the development and growing technical division of labour - the expansion of activities as well as the absolute numbers of people involved and goods and services produced.

As manufacturing activities have either grown absolutely smaller due to technological substitution, or migrated to lower cost labour locations, so the development of product differentiation has occurred. Thus, when Ford produced the 'Model $\mathrm{T}$ ' it was one model, in one colour. Now few consumers seem happy unless a product is available in a multitude of colours, materials and designs. One advantage for producers is that they can sell the same product more than once to a consumer, and another is that they can charge a premium for designer/quality goods. Thus, there has been a huge growth in what we might generally call design or customisation of products; moreover, as we can see from products like Apple's iPod, design may drive production and market share. Thus we can see how one new source of what business strategists and economists call 'competitive advantage' is creativity, or what we might more generally call innovation.

Accordingly, there have opened up many new opportunities for creative inputs to products. Thus, a larger proportion of manufacturing related activities are beholden to the creative economy. As we will see in moment consumer spending has also grown in this sphere; a critical aspect of consumer spending is that it is associated with youth, and a range of concerns about identity and culture. The birth/branding of the teenager was the start of a long boom that has continued to this day. The key shift is that cultural products once the realm of 'one offs' and 'live per- 
formance' are now readily reproducible millions of times (for the same economic input); this has led to a huge growth of cultural and creative industry producers (as we'll see later).

Production in the whole economy has become more extensive, production occurs in networks that do not simply link a few buildings and a factory, or a region, but across nations and the globe: globalisation. As I have already mentioned, one driver has been cost reductions. Initially locations in the world would compete for a branch plant, offering subsidies. Now, the global North, or global cities compete for the head offices of corporations. The tool for such competition is the 'unique selling proposition'; what could be more unique than heritage and culture, that is except when it's a modern art gallery in an old power station located by a river with a nice bridge across.

So, cultural consumption is the new honey pot to attract investor bees; a variation of this is the notion of the creative class (Florida 2002), that is not creative workers, but those who like to be around artists, whom city boosters think will attract they key labour, and which will be the magnet to attract high tech industry. As I'll point out later, this is a case of culture being used instrumentally to achieve other ends; moreover, it does not address the question of investment in cultural production.

An illustration of the scope of such changes is difficult, as I'll discuss more fully in a moment, as by definition most of these activities and goods are new, and thus they are missed by census makers and statisticians; they are quite literally invisible. Despite a number of caveats we can offer some measures of these industries, although more work needs doing to make them more precise. A recent survey carried out for the European Commission (Kea European Affairs 2006) for example shows on average $3.1 \%$ of the working population in this sector, and 2.6 $\%$ of European GDP, on a turnover of 654 Billion Euro; some countries such as the UK and Germany are way above these figures. Whilst, these figures may seem small when compared with those staples of the 'real economy' such as motor manufacture, textiles and chemicals looks small (See Figure 1). 
Figure 1: The Cultural and creative sector in Europe (2002)

\begin{tabular}{|l|l|l|l|}
\hline Sector & NACE classification & $\begin{array}{l}\text { Persons employed } \\
(1,000)\end{array}$ & $\begin{array}{l}\text { Number of } \\
\text { Enterprises }\end{array}$ \\
\hline $\begin{array}{l}\text { Manufacture of } \\
\text { motor vehicles }\end{array}$ & 34 & 2,163 & 160,834 \\
\hline Chemical industries & 24 & 1,929 & 31,421 \\
\hline Creative industries & N/A & 6,420 & $1,394,162$ \\
\hline $\begin{array}{l}\text { Manufacture of } \\
\text { electricity, gas, etc. }\end{array}$ & 40 & 1,181 & 14,880 \\
\hline $\begin{array}{l}\text { Manufacture of } \\
\text { machinery }\end{array}$ & 29 & 3,527 & 162,257 \\
\hline
\end{tabular}

Source: KEA European Affairs (2006)

As I have mentioned, hard data on key indicators is a tricky area, one that we are currently devoting much work to, so, comparisons outside of Europe are still patchy. However, as an indication we can look at the contribution to GDP in a variety of countries; we can see that Europe is not alone in this phenomena. I am not here to explain or explore this phenomena today; what I would point out - and will touch on again later - is the uneven spatial distribution of the gains from the cultural and creative industries, both across nations, and within them, and even between cities and regions

\section{Culture}

Culture has changed in a wide variety of ways. In a most obvious way we can see that cultural forms are constantly developing and changing. Noticeably, this process is accelerated and intensified through feedback and critique. Thus, the development of various markets, or schools of cultural criticism has driven the development of forms, and the expansion of new forms. One of the key elements of culture centred on the developed world is that parts of culture have become traded, or marketised. As noted, already, hand in hand with mass production and mass consumption this has led to huge demand for cultural goods and services. As I mentioned, new consumers (younger) are constantly drawn in, as is the rate of turnover of fashion/values or taste.

We can find some evidence of this shift in statistics on household spending patterns As a whole developed societies are getting richer (although not more equal), and a greater proportion of spending is being directed to cultural goods and services. Whilst there are international contrasts; the growth and scale is significant. I haven't got time to explore the participation rates and time spent on cultural activities, but this would be another piece of evidence to add to the picture. 
As noted above, culture is changing; perhaps an emblematic debate has been that of high versus low culture that resonates through many societies. This debate has become more complex in that it used to map onto state funded and market provided; however, this division has been eroded, as has the certainty of which categories particular art forms fall into: these categories are not 'eternal' but historically and culturally specific; for example, Adorno cast photography, jazz and film to 'low' culture. Whilst one may have sympathy with the argument that 'true value' is not captured by economic prices; it does not follow that if something has a price then it has no cultural value. The negotiation of this 'grey' area of cultural and economic values is clearly problematic (and carries with it much baggage of old debates), and as such it provides policy makers with less and less guidance as to which cultural forms to support, or not; let alone how. When classical music is selling CDs and on commercial radio, and commercial concerts is there a need for public support? How should it be justified when market failure appears not to be present? I think that this is dangerous territory; we need to navigate these areas very carefully. Increasingly we can note that cultural activities can be found across a number of boundaries: cultural and creative, commercial and non-commercial, formal and informal economies, and across production and consumption. As noted above this last point is a critical change away from the producer creating a product and placing it on sale then hoping to convince the customer that they need it. Now, we see an intense short-circuit and feedback between production and consumers with so called 'cool hunters', urban anthropologists feeding back the street to designers (Quart 2003).

\section{State}

The third of our three realms of empirical change is the state itself; as we are all aware the notion and role of the state has changed much in the last 50 years from a high point of social welfare to the current engagement with the minimal state of neo-liberalism. We have seen state spending fall as a whole, and much concern by politicians of all shades to examine a much smaller state, either from ideological terms, or simply as a way of responding to a shortage of money. In these circumstances it is not surprising to see the fact that the arts and culture budget is one that is easily cut, compared, for example to education or health although in many states they have suffered as well.

In such a shift we can see changing modes of resource allocation, and a move to 'value for money' evaluation. Again, in standard terms culture is always going to loose. This is perhaps why there has been 
such an upsurge of interest in finding other rationales for the support for culture, and the expansion of instrumentalism arguments for the existence of culture. I feel that some of these arguments still need development; they certainly need to be based on more robust evidence either statistical or explanatory. The sad fact is that although we have now begun to recognise the role that both the cultural economy, and the contribution of culture to social improvement in direct and indirect ways, we still only have a very partial understanding of what constitutes the cultural economy, and how it works, and what its relationship to the rest of economy and society is. The following section develops this argument showing how a re-appraisal of concepts, making and governance can be developed.

\section{Re-defining 'Concept-making-governing'}

\section{Reconceptualising Culture}

One of the most important steps taken in recent years in this field has been the attempt to 'measure' the impact of culture on economy and society. Initially, the focus was on so-called secondary impacts, or economic multipliers. These modelled the 'knock on' effects of culture seemingly admitting that adding a value label to culture was not possible (Throsby 2001). So, things like expenditure for hotels and restaurants were see as a measure of cultural impacts. The key oversight here is that cultural production is not examined, and the only worth is considered to be consumption related (but even then disconnected with cultural production which is rendered invisible).

Thus the significance of the shift to primary measures of cultural activity; measures that included the makers and artists, but, and this is the critical point, they also considered the related activities and jobs needed to facilitate cultural production. Just as one would not evaluate the contribution of the car industry from counting is sales staff; or, one would not evaluate the contribution of the film industry only through the star actors.

However, the argument goes further than this, it is concerned with all of the activities required to produce a cultural product or service. That involves what have been termed the depth of cultural production (that is the behind the scenes work), as well as the breadth - that is how widely one defines culture (Pratt 1997; Dcms 2003; Burns Owens Partnership, Pratt et al. 2006).

Empirically, what these approaches have sought to do is to capture the social and economic, the formal and informal, commercial, non- 
commercial, as well as the production and consumption activities that constitute cultural production. Moreover, examining these processes, through the interlinked networks of production, has highlighted the international flows of cultural production (not simply the flow of exports), as well as the dramatic clustering, or co-location, of parts of these industries.

We have already seen examples of the output of these models, and the diagram here gives one a sense of the scope of activities under consideration to create a full picture and understanding of the cultural economy. As noted, much of the work so far has concentrated on traditional measures such as output, employment and occupation; little attention has been paid to processes. Of course a firm basis for policy making has to be rooted in an understanding of causes and process, so that the best intervention can be developed - to be effective and efficient.

\section{Re-making Cultural Industries}

There are three main characteristics shared by all cultural industries, as well as some non-cultural industries, and the manifestation of each is different by different industries that have different market structures: for example, computer games and the film industry, or the theatre.

The first is the general organisational form. Most of the cultural industries are dominated by a handful of major international corporations, and sitting below them are many thousands of 'companies', these companies are very small, indeed micro-enterprises comprising of self employed and 2-3 person business. There is a 'missing middle' or small and medium sized enterprises, which leads to some challenges in terms of co-ordination. On the other hand it develops a network, or rather an interdependent 'ecosystem' of companies that are constantly growing and evolving into other companies recycling knowledge, expertise and personnel. Like any ecosystem it is delicately balanced.

Second, is the work process. As already suggested the 'life' of products and projects to create them, and the firms that produce them, are short: a matter of weeks or months. These 'firms' are constantly recycling and evolving; they constitute a whole sector of the economy that has a 'project based' form. As such, traditional analyses that treat the firm as a basic building block of analysis are not so helpful as those that see the more enduring 'network' or 'institution' as more salient. Once again, I can only sketch out some outline here, the richness of detail of organisation and practice would take all the time I have here today to relate. 
Third, is the rapid turnover of products and sequence of multiple innovations required to sustain activity in the sector; added to which there is incredible uncertainty that when a product reaches a market, or an audience, that they will even like it. The critical element of timeliness is crucial. So is the act and co-ordination of market building through education or publicity. Hence, consumption feeds back into production and both are attenuated by micro-differences. However, success of failure depends on such differentiation as this is an industry where the winner takes all; coming second is really no good.

These characteristics, separately and in combination, are difficult to comprehend and fully understand, and, as I already mentioned they are subject to rapid change and variance across cultural industries. This really does create a substantial challenge for policy makers.

\section{Re-making Governance}

So, taking into account the changing contexts, cultural economy and concepts what is the future for cultural governance: a free market? To be sure, this is the concern of many; especially, in those states that have a long history of support for culture and have built substantial orchestras and art galleries, etc. My concern is that business as usual in policy terms will lead to the public sector having a dwindling control over spending, and over the operation of the cultural sector. Thus, in order to simply stand still we need to re-calibrate our response, and in some ways entirely change the way that we manage cultural activities. I'll come back to what are inevitably future challenges in my conclusion; what I want to cover briefly in this section concerns the current state of the art.

The current cultural policy field is rather limited; it has expanded upon its original remit in two areas. Crucially, whilst traditional cultural spending has declined, two new areas have become the new targets: First, instrumental policy aimed at using the feel good factor, or the actual capacity of the arts and culture, for 'regeneration': which might mean using a cultural quarter to regenerate a downtown, or peripheral estate; or, using art projects to improve social inclusion. Second, and perhaps most saliently, the use of arts and cultural facilities to attract inward investment/foreign direct investment into cities and regions.

The second field where cultural policy has expanded concerns the direct economic impacts of culture. Thus, measures of intellectual property rights, royalties, trade, and employment, as well as the impact of the rest of the economy are seen as key issues. This second field has the most to offer the cultural and creative sector; but as yet policy makers do not seem to have developed means and understandings of the workings 
of the industries in order to devise policy. On one hand there is generic industrial policy, on the other hand censorship: few examples of dedicated policy exist. This is an area where more research could have a big impact.

One of the problems with this second area is that it is commonly identified solely with the commercial sector, and in opposition or competition for public funding with traditional arts and culture. This, I believe, is a mistake. Empirical observation tells us that one of the characteristics of many artists and cultural workers is a constant migration between the for-profit and the not-for-profit sectors. This, I would argue, needs to be central to our concerns rather than, as it is at present, missed by both.

\section{Conclusion: Future Challenges}

This paper represents a sketch of the challenges facing policy making in a new field, it complements other papers that I have written on the topic (Pratt 2005). In my conclusion I want to briefly raise a few challenges that the rise of the cultural industries, and the problems that those developing policies for them confront. First, I'll recap the argument: that cultural production has changed, so has the nature of culture and the role of the state. The old means of managing culture are no longer applicable; we need new ones. Moreover, an incremental shift, or business as usual will not suffice. Radical changes are needed - but what changes? I have argued that we need a fuller understanding of the cultural economy and how it operates in order to effectively govern it. The policy must go beyond instrumentalism, or simple subsidy, it requires an intelligent and nuanced policy approach, and moreover, it needs a new cadre of policy makers to implement it. Elsewhere I have argued that these 'intelligent agents' may need, like the industries that they intend to support or encourage, to be from the public and private sector, formal and informal economy: they need a very diverse and flexible approach one that will require the skills of a simultaneous translator skilled in the languages for art, culture and design, as well as politics, administration, economics and state budgets (Jeffcutt/Pratt 2002; Pratt 2004).

I have pointed to the increased questioning of old hierarchies and assumptions about cultural value and universalism; I have countered this with the idea of a situated (in time and place) democracy. Such a strategy presents a challenge to the old 'allocative' model of supply subsidy. It does not fall into the trap of suggesting that market allocation is best either. Instead it opens up a new field based upon a complex and medi- 
ated interaction of 'supply and demand'/'production and consumption'/ 'artists and audiences'. It is this new agenda that I argue that we need to articulate to if we 'care' about arts and culture. Doing nothing is no longer an option. However, I do not underestimate the practical and institutional challenges that a re-framing of cultural governance in this way implies.

I will end this chapter with an oversight of some of the problems that will face this new agenda and policy community. As I have already mentioned, people and organisations who can work at the boundaries of the commercial and non-commercial, and are happy to move in and out of one or another. Second, state agencies will need trained and trusted individuals to broker these relations and policy - most likely some sort of third sector agency might work best. Third, we need to resolve our concern for and objectives of supporting culture. The old formulation, the one that I began the chapter with, plays into market failure; the new one is potentially simply a free market.

However, I also see another argument which depends upon a more subtle understanding of cultural production and cultural value making, one that is able to seem collectives and well as individuals, the role of the private individual as well as the society, the role of the instrumental as well as the 'arts for arts sake' perspective.

Fourth, regardless of policy making it does seem that we already have a huge cohort of workers in the cultural sector that are precarious or freelance labourers; they may, some of them, earn good money (some of the time) but they have no certainty. If we are to sustain livelihoods in this sphere we need to develop a compatible social welfare system that supports it, not one built upon the idea of male workers in a career for their working lives. Fifth, we need to recognise that we cannot all be 'winners' in the culture wars - as it is being played out with economic overtones some regions of the world are great gainers from this competition; many others losers.

Underpinning this is that fact that the cultural industries are massively dominated by a small number of companies who critically have control over what gets to audiences and markets. This poses a challenge to notions of cultural democracy and representation. Nowhere is this more evident in the developing world where the supposed 'level playing field' of Intellectual property rights (IPR) is working as a means to ensure that cultural producers cannot participate in profits; and of course create livelihoods, and economic and cultural wealth. However, this is a topic that must be the subject of another chapter. 


\section{References}

Adorno, Theodor (1991): The Culture Industry: Selected Essays on Mass Culture, London: Routledge.

Baumol, William J./Bowen, William G. (1966): Performing Arts - The Economic Dilemma: A Study of Problems Common to Theater, Opera, Music and Dance, New York: Twentieth Century Fund.

Bourdieu, Pierre (1979): Distinction: A Social Critique of the Judgement of Taste, London: Routledge.

Bourdieu, Pierre (1993): The Field of Cultural Production: Essays on Art and Literature, Cambridge: Polity Press.

Burns Owens Partnership, Pratt, Andy C. (2006): A Framework for the Cultural Sector: A Report for UIS/UNESCO, London: BOP.

Crandall, Robert W./Sidak, J. Gregory (2006): Video Games: Serious Business for America's Economy, Washington: Entertainment Software Association.

DCMS (2003): Regional data framework for the creative industries: final technical report for the Department of Culture, Media and Sport and the Regional Cultural Consortia, London: Department of Culture, Media and Sport.

Florida, Richard (2002): The Rise of the Creative Class: And How It's Transforming Work, Leisure, Community and Everyday Life, New York: Basic Books.

GLA Economics (2002): Creativity: London's Core Business, London: Greater London Authority.

KEA European Affairs (2006): The Economy of Culture in Europe, Brussels: European Commission DG5.

Pratt, Andy C. (1997): 'The cultural industries production system: a case study of employment change in Britain, 1984-91'. In: Environment and Planning A 29, pp. 1953-1974.

Pratt, Andy C. (2005): 'Cultural Industries and Public Policy: An Oxymoron?' In: International Journal of Cultural Policy 11, pp. 31-44.

Quart, Alissa (2003): Branded: The Buying and Selling of Teenagers, Cambridge/MA: Perseus Publishing.

Throsby, David (2001): Economics and Culture, Cambridge: Cambridge University Press.

Wasko, Janet (2003): How Hollywood Works, London: Sage. 
Creative Governance 



\section{Reflections on a Governance Model for Creative Industries}

PERNILLE ASKERUD

We are witnessing the industrialization of culture

on a global scale - entering a time when the creative arts skills will be right at the centre of wealth creation, and thus of business, and thus of governance.

Simon Evans, speech delivered at the Creative Clusters Conference $2007^{1}$

\section{Introduction: The Creative Economy}

Together with the increasing importance of information in the global economy, the notion of the creative economy has emerged and is generally accepted as an increasingly important part of economic activities worldwide. Structural changes associated with the creative economy are engendering new opportunities as well as patterns of exclusion and inequality that challenge existing governance models.

The dominating importance of information in the global economy makes it imperative that concepts of culture and creativity be reassessed and repositioned at the centre of public policy. The proliferation of ICT has brought with it a gradual but fundamental change of the way culture is perceived by societies at large, especially in their role as consumers of cultural goods and services. The mobilization/involvement of larger

1 Simon Evans, speech delivered at the Creative Clusters Conference 2007. 
segments of the society in cultural activities is associated with increased demands for support to an ever growing number of cultural institutions, projects, and individuals.

While aware of the growing economic importance of culture and information, many countries are, however, uncertain just how to engage proactively with the culture sector as part of economic development plans. Overall, there is a feeling that traditional ways to support culture are perhaps no longer sufficient but the lack of data on other aspects of culture than fine arts and heritage (in relation to which information has primarily consisted of inventories and archives) hampers a wider analysis of the sector's needs. Similarly, the crafts and artisan sector remains largely outside the realm of Government planning and intervention/support.

The businesses and industries that make up this new creative economy have given rise to differing or overlapping definitions with creative industries, content, knowledge or copyright-based industries, and cultural industries, as some of the main categories applied. In everyday usage these terms are often used almost interchangeably though there is a growing consensus on the different connotations and perspectives implied with each of these terms. Within this consensus the cultural industries are separate from creative industries or even embedded as a particular segment within the wider notion of creative industries. What is common for all the 'new' industries covered by these categorizations is that knowledge, education, creativity, and intellectual property, are very prominent features of product development and profit.

As economic and cultural policy must become ever more integrated, the need to understand the sector better has become pressing. In the following, we will look at the ways creative industries are understood in separate contexts, and the different interests and policy perspectives they engage. Taken together they represent society's increasingly complex perception of the role of culture and creativity in the spheres of both economy and civilization, and provide elements to the identification of a new governance model for the creative economy.

\section{Four Discources on Creativity and Economy}

Creative industries are also referred to as cultural industries, knowledge industries, information industries, copyright-based industries, etc. Behind this semantic confusion there are at least four distinct discourses within which the use of the terms is understood differently. 


\section{An Economic Discourse}

The economic discourse is probably the simplest and to a certain extent the best known. The economic discourse is an expression of mainstream economic development in recent years and is closely connected to the ideas of globalization and free trade. The very high growth rates reported from the knowledge or creative economy in recent years stem primarily from studies based on this wider concept of creativity. The universal acceptance of the economic model of creativity is expressed in the progress made towards identifying standard indicators for the socalled copyright-based, creative industries.

The economic discourse operates with a very broad definition of creativity and creative industries centering on copyright and other intellectual property rights as a key to profit and economic growth. A model developed by WIPO $^{2}$ illustrates this approach to creativity. The model includes four categories of creative industries where the 'core copyright industries' and the 'partial copyright industries' correspond best with other definitions of creative industries, and the 'interdependent copyright industries' (e.g. TV and broadcasting) and the 'non-dedicated support industries' (e.g. ICT and transportation) indicate a much broader economic approach to these industries that is only loosely linked to culture and artistic creativity. ${ }^{3}$

Many of the most successful cities throughout the world have adopted this economic model of creativity as a base for economic development strategies and have invested heavily in the development of largescale projects planned to accommodate the new creative industries in the form of 'creative industry parks' or other infrastructure investments much as one would support any other industry. In many cities, however, there often seems to be a gap between the articulated and official policies concerning culture and creativity and their implementation. The use of cultural districts in economic development plans provides good and tangible examples of this discrepancy. Hence very few projects acknowledge the complexity, resources and diversity that foster unique cultural districts, and the ability of their cultural uniqueness to enhance the quality of life and attract tourists and others is far too often taken for granted. The associated rapid modernization process, the scaling-up of activities, the top-down urban renewal, and the measures of cost-

2 WIPO: the World Intellectual Property Organization.

3 Source: Guide on Surveying the Economic Contribution of the CopyrightBased industries, WIPO, Dec. 2003) see: www.wipo.int (31.1.2009). 
effectiveness, associated with this development is often at the expense of or even contrary to the needs and interests of the very culture and environment that is necessary to nurture the creative industries (Youn 2007). The development of creative industries in this context, therefore, also emphasizes the problems associated with urban migration: slums and urban poverty; environmental degradation; waste; the need for people to leave their homes for long periods of time to seek work, and the consequent breakdown of local communities.

\section{An Urban Development Discourse}

The urban development discourse originally emerged in European cities as a strategy to combat unemployment and urban poverty in localities where the old heavy and manufacturing industries no longer provided the economic basis and employment opportunities as before. The strategy focused on the generation of jobs, especially through new technology-based information industry development and the fostering of more attractive cities. How this attraction is defined differs from place to place but the concept of creativity embedded in this discourse is more closely linked to the notion of culture than it is in the economic discourse. Enterprises built on the new technologies such as design and new media enterprises were an important strategy for example for the redevelopment of many European urban areas in the 1980's and 1990's. Today, the urban development discourse continues to capture the reorganization of power and space in the global economy, especially in terms of the adaptability and flexibility required by the new, constantly changing economy, which the cities are particularly well placed to respond to.

The key quality of culture in this context is its ability to improve the quality of our lives and surroundings by making them more stimulating and filling them with beautiful and stirring objects and in the process making them more diverse, interesting, and attractive for creative industry development and talent migration. The discourse is imbued with certain values and life style ideals and focuses on the individual as a source of creativity and innovation, and on the creative industries as a particular attractive opportunity to cultivate and free this 'creative capital', expressed in its most radical form in the notion of the 'Creative Class' (Florida 2002).

Within the context of urban development, the approach is essentially not too different from our intuitive understanding of the concept of culture and related industries. Here the line between art and commerce tends to be ideological rather than analytical and so the discourse tends to encompass a wider spectrum of policy goals. Creative industry devel- 
opment is often considered part of the inherent dynamic of urban spaces, and it is indisputable that the urban environment provides the ideal conditions for the kind of clustering that sustain many successful creative enterprises. The urban development discourse tends to define cultural or creative industries - enterprises whose core activity, whose value is based on a traditional cultural skills: singing, dancing, acting, telling stories, making images, in a word: art and culture, empowered and organised by technology and management, and cultural goods whose primary economic value is derived from their cultural value.

Despite the importance of place highlighted among other by followers of Richard Florida's theories, the high tech opportunities created by the new economy are not limited to the concentrations in cities and to dedicated regional developments. Capitalizing on strengths in on-going research and educational institutions and supporting the birth and growth of innovative enterprises, locations around the world are developing high tech [creative] sectors.

Some of the major urban centres in the world, such as the port cities of Hong Kong, London, and New York, have long been dominating the international economy and have been main factors in the globalization of the world economy. This development has to a great extent built on already existing economic activities in trade and international finance upgrading and strengthening these by the strategic employment of information and technology.

Recently, they have all presented themselves as creative cities hence demonstrating a slide towards a more culture-based understanding of creativity and creative industries. Different models have been adopted in other cities throughout the world where technological development have been strategically employed to adapt or revitalize old manufacturing industries within the cities.

The initiatives that cities are developing and carrying out to engage with the new economy are, in other words, as varied as the cities themselves. Hence, the notion of the creative city has also inspired cities to re-invent themselves, often by raising architectural monuments as a way to shape and demonstrate identity, competence and, not least, economic power - this is true for European cities like Bilbao, Berlin, Paris, London, and for New York, Beijing, Shanghai and Hong Kong in China and perhaps most of all for cities and 'new' destinations like Abu Dhabi and Dubai. 


\section{A Discourse based on Notions of Cultural Diversity}

Though it represents a challenge to cultural diversity, the globalization of economy and trade have also created the conditions for a renewed dialogue among cultures and civilizations based on human rights and respect for their equal dignity. Hence, in parallel with the growing focus on creativity and information-based industries as key investment areas of the future, there has been a growing recognition of the potential and importance of culture and, especially, of cultural diversity as a strategic component of economic growth and competitiveness. The shift in the definition of culture itself also reflects a heightened recognition of the importance of cultural diversity as a key to leveraging competitive advantage.

The concept of 'cultural diversity' stipulates that plurality is the necessary requirement for freedom and that in political terms such pluralism is inseparable from a democratic society. It is in many ways as fundamental an expression of our time's perception of humanity and civilization as the concept of human rights. The very concept of cultural diversity has, however, been the topic of much heated debate - especially in relation to the economic aspects of culture with the discussion of the extent to which cultural goods and services are different in kind from other goods and services (the cultural exception) being a focus of contention. Differences of opinion have pinned the US against France and Canada, and the regulatory authority of WTO against that of UNESCO and WIPO.

The linkages to cultural rights on one hand, and trade issues on the other, make cultural diversity a particularly difficult issue in terms of cultural policy. As a resource for innovation and creativity cultural diversity is of course linked to the creative capital necessary for creative industry development. The disagreements on definitions and terminology, however, emphasize some of the complexities surrounding the concept of the creative industries and their implications in different ideological and economic contexts and the various underlying discourses described here. They also point to the inherent contradiction between market interests of nation-states in creative industries as a factor that may, quite against the intention, lead to the harmonization of the diversity concept and the consequent marginalizing of cultural minority groups (ibid.). 


\section{A Discourse on Culture and Sustainable Development}

Finally, the notion of culture as an inalienable component of sustainable development focuses, on the one hand, on principles such as participation, aspiration, and diversity as the success criteria for any development project while, on the other hand, it opens up the sphere of culture to the economic transactions that are part of cultural industries. Creativity and cultural industries have therefore also become a key concept in discussions of sustainable development, especially in developing countries.

As part of the development agenda, examples of creative industries or cultural industries as is the preferred terminology in this context - are most often seen as small-scale community-based industries such as cultural tourism, crafts, music, and heritage preservation - in other words a more narrow perception of culture and creativity than found in the policy debates of many industrialized countries.

Within this context, the business unit in the creative industries sector is (in contrast to more traditional, larger scale industrial development) typically quite small, often family businesses ${ }^{4}$, which means that the product origination takes place in many, diverse units while the production mostly is on a smaller scale. In this respect, the cultural industries are particularly suited to contribute to community regeneration and correspond well with the practice in more traditional societies.

Offering not only the possibility for income generation, the cultural industries provide opportunities for employment that are easier to reconcile with family and community obligations. Cultural industries need qualified and informed workers and stimulate the upgrading of such skills and knowledge. The creative process in itself encourages participation, which spills into the community at large.

By recognizing the social impact of cultural industries not just as an output attribute of the consumption of these goods and services but as an end in itself, it becomes possible to link support for cultural industries to strategies for social/community regeneration as a development goal.

4 Obviously, this is in particular true for the start-up phase of many Creative Industries and more generally for the cultural industries and does not apply in the same way to the larger-scale Creative Industries in for example the media and leisure sub-sectors. 


\section{The Re-Evaluation of Creativity in the New Economy}

What we observe in the context of this new economy is the emergence of distinctly different modalities and opportunities for work, increased participation of the non-formal sector, new business models and forms of capital - as well as the erosion of traditional organizational and social patterns where many more people are self-employed and some are excluded. These changes, which also include new consumption patterns related to cultural goods and services, are in fact the effects of fundamental, structural changes in the international economy and the way we work and live, comparable to the changes that followed the industrial revolution.

Over the last decades, the distinction that existed between culture and more traditional concepts of manufacturing industries is breaking down. We are moving towards an understanding of culture and creativity that centres on the productive and innovative capacity of knowledge and information - rather than a more traditional concept of culture as linked to the classical or fine arts. In the new economy, creativity has become an enterprise sector, which holds great potential for economic growth, market expansion and profit generation. These trends are observable throughout the world in many different forms, altering the existing patterns of dependence and dominance, under-development, and the regional inequalities determined by the relationship between the new centres and peripheries in the international economy and trade. These developments have, naturally, put a new emphasis on policy development for creativity in recent years.

Yet in order to successfully promote creative industries as a sector of the creative or knowledge-based economy, the context of a place as well as the differences between the discourses described above and the industries they focus on must be considered carefully. There is a tendency to overly simplify the kinds of characteristics that are important in general to attract and nurture successful creative enterprises and to consider the impact of the creative economy exclusively as an urban development issue.

Smaller economies would therefore seem almost by definition to be barred from participating in the creative economy. Nevertheless, because the creative economy is not just a question about arts and culture-based industry development but a much more comprehensive and fundamental phenomenon of the global economy, smaller economies wherever they be simply must relate to these new economic trends and formulate strategies for survival and growth - or risk to be permanently left behind 
or excluded from the economic changes taking place, predominantly in the more dynamic urban economies.

While the creative or information economy affects the global economy everywhere, the strategies for the promotion of the creative industries have largely sprung from urban areas where the ready availability of a wide range of skills and human resources, more sophisticated technological infrastructure, and easy access to information and other resources needed in the creative industries have allowed a minimum of policy intervention, and investments to be directed either towards status bearing and iconic projects, or towards business incubators and other mechanisms aimed at strengthening the emergence of new business enterprises. Other aspects of the policy environment not directly aimed at creative industries such as the deregulation or regulation of markets and labour, or investment in infrastructure, have different impact and meaning in different contexts determining the viability of creative industries development in a particular locality or region.

Hence, the policy environment and investment strategies that allow engagement with the creative economy depend on the resources, identity and potential of a locality or region and are therefore not the same everywhere. A few examples: while it is true that access in a location to a high quality workforce is certain to attract enterprises not all businesses in the creative economy are dependent on highly qualified staff or attractive locations - as demonstrated by the call centres and back office services. In India and in other places, these industries provide an excellent opportunity to tie the benefits of the new economic growth to a wider range of the population. Similarly, it is often stated that a very high percentage of people engaged in the creative industries are self-employed. While this may be true for some places it is only true in certain contexts - as indicated by the proliferation of multi-national companies such as Time-Warner News Corporation (the world's largest media conglomerate) and the Walt Disney Company, Vivendi, Microsoft, and large companies like for example Nintendo, Lenovo, Acer, and Sony, that drive the creative economy in many East-Asian cities and elsewhere.

Whereas large multi-national companies such as these are very profitable and run their business much like any other industry, many activities especially in the cultural industries may appear to have only a limited commercial potential. Similarly, the large multinational creative enterprises would perhaps seem to have very little interest in cultural policy while other segments of the creative industries are much more directly dependent on policy support and investment. Traditionally, funding and programming in the cultural sector have been directed towards projects, institutions, and large enterprises, all heavily dependant on 
public initiative and financing. The sustainability of the creative industries depends, however, on a different and more comprehensive model of investments.

In fact, in many countries most of what could be considered creative industries is part of the non-formal economy. Many of these enterprises lack the capital needed to scale up their activities and operate as demand-driven sustainable businesses. Major problems in this context include that the entrepreneurs in the creative industries seldom are able to present a convincing business model, and that many of the professional skills involved - e.g. choreography, dancing, drawing, editing, weaving, doll-making - simply are not perceived as leading to profitable businesses. While this may be changing in some places under the influence of success stories of one kind or another, many skills and professions related to the creative economy are not even recognized as business categories in legal terms.

Consequently, in many countries, small creative industries do not have access to credit facilities or to the loans and investments that would make their businesses more viable. By main-streaming and professionalizing the non-formal sector and the traditional professions, it would become possible to extend privileges and support mechanisms as they apply to other sectors of the economy, for example in terms of more general recognition and access to financing that would allow the producers to build-up the necessary stock to be able to trade in a different manner and reduce their dependency on brokers, and in the process ensure a sounder basis for business development and a more equitable reward. ${ }^{5}$

Creative industries, like any other industries, are only truly sustainable if they contribute to building the asset resource base. This they can only do through the integrity and authenticity of their products. In the long run, the creative industries depend upon the vitality and strength of the culture sector and the investments in cultural capital (knowledge and heritage) and so cultural policies are important for all segments of the creative industries. The sustainability of the creative industries therefore depends on the safeguarding of cultural capital and the development of

5 Singapore is currently implementing an integrated continuing education and training system, the Singapore Workforce Skills Qualifications (WQS) system, which aims to identify the need for specialized training and accreditation needed to boost development of industries through the systematic enhancement of workforce's employability and competitiveness. The Creative Industries Workforce Skills Qualifications Framework (CI WSQ) is a component of the continuing education and training activities programme that target skills development for the creative industries in particular. See also Stöber/Ooi in this volume. 
the cultural asset resource base. Hence, comprehensive cultural asset management is a prerequisite for sustained growth in the creative industries sector - and, in a wider perspective, for sustainable economic development and vibrant community life. It is therefore necessary to maintain the principle that cultural assets are inter-generational capital and that their viability may legitimately be sustained by public investment.

All of these factors and many others are part of the context that determines the viability and investment needs of different creative industries. Policy decisions need to balance the requirements for the cultivation of a sense of identity with economic and other policy objectives. These decisions depend on the context - the location, resources, values, aspirations and identity of a particular location and community. For any individual company or city, greater specificity is therefore needed. Companies vary in their location requirements depending on what exactly they are doing and their place in the chain from research to production. Planners and policy makers need to understand such differences between companies as well as a region's or city's own resources and potential in order to focus on realistic development possibilities and identify an appropriate and effective strategy.

Creativity and innovation feature in all creative industries but the interventions needed to stimulate development and the overall goals may also differ depending on the social context. Rural areas and not-somodern urban areas often display a differing set of values and aspirations from the technology-rich urban centres, and the development of creative industries as part of the economy will consequently take different forms. As the creative economy gains momentum also outside the urban mega-cities it is quite possible that we will see a diversification of the values and norms that is typically associated with the creative economy.

\section{Policies that Work}

So what are the elements of a governance model for the creative economy? What are the goals and dreams for an aspiring creative city? What are the implications for economic and social development in poor areas? Concerns and priorities such as employment opportunities for all, economic growth, and cultural vitality in a sustainable environment that incorporates history and culture with innovation and beauty - how are these goals best achieved?

Most of the international experience on creative industries development cites the need for innovation, flexibility and efficient cooperation 
with authorities as key thereby highlighting the difficulties associated with the cross-sectoral character of creative industries development. Most important of all, is the need to adapt a sufficiently wide policy framework to accommodate the great variety of scenarios as demonstrated in the policy models briefly referred to below.

Richard Florida's prescription for creative cities - vibrant, aesthetically appealing urban settings with workable transportation systems and affordable neighbourhoods - is support for the Three Ts: talent, tolerance and technology as drivers of creative industry development Florida 2002, 2007). Florida's Creativity Index model was later adapted by Hong Kong to reflect different values and policy objectives by including and emphasizing the importance of the cultural and social capital in creative industries development in addition to the three drivers identified by Florida.

As part of an analytical framework for cultural industry sector analysis UNESCO has identified five drivers of culture-based creative enterprise development. The drivers are key agents of change in very different kinds of environments and include: 1) social organization and values (e.g. press freedom); 2) human resources development; 3) cultural asset management (e.g. access to information); 4) technological development; and 5) infrastructure and policy environment. The drivers are.

These policy models demonstrate governance models for creative industries development. As the role of culture change, the need for administrative change to accommodate the integration of cultural policy and economic policy will become more and more important; any governance model for creative industries will need to reflect this. In this new context, the usefulness of cultural indicators will depend on the extent to which they allow policy makers to evaluate the environment needed for cultural and creative industries to thrive.

\section{Perspectives}

Mostly, when we discuss creativity it represents something good and positive - a complex package of cultural, social and, increasingly, economic opportunities. But for many people around the world the rise of the creative economy raises the spectre of new inequalities and social indignities where creativity is reserved for the others, where work, if it can be found, is not creative at all, and where the cities are ghettoes of slum and poverty.

A gulf is opening up between the beneficiaries of this new economy and the so-called creative class - urbanites who are articulate, speak 
English and other international languages, have access to continuing education, understand the internet, are confident with the shorthand of global branding and celebrity, - and the emerging non-creative underclass.

The challenge for any governance model for the creative industries is to recognize the more comprehensive administrative restructuring that is part of this development, and ensure a governance model for creative industries that is sufficiently complex to address these unintended results associated with the creative economy as well.

\section{References}

Florida, Richard (2002): The Rise of the Creative Class. And How It's Transforming Work, Leisure, Community and Everyday Life, New York: Basic Books.

Florida, Richard (2007): The Flight of the Creative Class: The New Global Competition for Talent, New York: Collins Business.

Youn, Elise (2007): A Comparison of Cultural Districts in Seoul. Paper presented at the Conference When Creative Industries Crossover with Cities, Hong Kong: Hong Kong Institute of Planners. 

Next City 



\section{Just Say Yes: Anmerkungen zur Governance von X-Cities}

\section{BERNHARD KRUSCHE}

Ein merkwürdiger Titel, oder? Zunächst mehr Wortspiel als Orientierungshilfe. Und doch verweist die an den Titel eines wunderbaren Aufsatzes von Tom Peters (2003) angelehnte Überschrift auf die Kernfrage der hier angestellten Überlegungen: Wie könnten - jenseits eines (noch) allgegenwärtigen Planungsparadigmas - Steuerungs- und Koordinationsformen einer zukunftsfähigen Stadt aussehen? Unsere Einstiegsprämisse: Wir gehen davon aus, dass sich Städte (ähnlich wie zur Zeit Unternehmen) in Zukunft stärker als bislang mit dem Umstand auseinandersetzen müssen, in ihrer Struktur, Funktion und Entwicklung auf Berechenbarkeit und hierarchische Ordnungsprinzipien $\mathrm{zu}$ verzichten. Für die damit verbundenen Ungewissheiten steht das X als Platzhalter. Gemeint ist damit weniger ein Rückgriff auf die Publikationen des OMA unter Rem Koolhaas (1998), sondern ein Kunstgriff auf die Unbestimmtheit zukünftiger Entwicklungen des Städtischen, mit dem die Zukunftsgestaltung von Stadt auf Grundlage unklarer Rahmenbedingungen markiert werden soll. X-Cities sind Städte, die sich der eigenen Nichttrivialität stellen und damit auf die Suche gehen nach Regeln, die im Zusammenspiel aller dabei relevanten Akteure Sicherheit durch Robustheit ersetzen. Welchen Prinzipien solche zukunftsfähigen Formen einer Governance folgen könnten, untersuchen und verallgemeinern wir an dem konkreten Beispiel von „Graz-Reininghaus“, dem zur Zeit größten privaten Stadt(teil)entwicklungsprojekt Europas. Hier wird seit mehr als zwei Jahren daran gearbeitet, aus der klassischen Planungslogik auszubrechen und durch ein der Komplexität von Stadtentstehung angemessenes Verfahren zu einem ,lebenswerten Stadtteil mit Eigenschaften“ zu 
kommen. Ein Blick auf die dort verfolgten Prämissen mitsamt der Herausforderungen, die sich in den konkreten Umsetzung im Zusammenspiel mit der Stadt und weiteren Stakeholdern immer wieder aufs Neue ergeben, zeigt exemplarisch Möglichkeitsräume auf, wie die Governance einer X-City nicht nur auf dem Papier aussehen könnte: Ein nicht abschließbarer (offener!?) Prozess, der mit der eigenen Überraschungsfähigkeit punktet, ohne sich dabei mehr als nur partiell selbst zu verwirren.

Wir gehen bei unseren Überlegungen davon aus, dass Stadt immer auch gebaute Gesellschaft ist. Für diesen Fall lohnt der Blick über den Tellerrand stadtplanerischer Prämissen hinaus auf die Anregungen und Hinweise, die auf der Grundlage soziologischer, kulturwissenschaftlicher und medientheoretischer Überlegungen die zukünftige Entwicklung von Gesellschaft (und man ist geneigt zu vermuten: einer Weltgesellschaft) diagnostizieren und kommentieren. Für diesen Blick greifen wir auf die Brillengläser von Dirk Baecker zurück, der im Fahrwasser medien-, kultur- und systemtheoretischer Zusammenhänge über die charakteristischen „landmarks“ (präziser: „socialmarks“) einer nächsten Stadt nachdenkt (Baecker 2004; 2007; 2008).

\section{Stadt als gebaute Gesellschaft}

Wenn wir das Wort von der Stadt als gebaute Gesellschaft ernst nehmen, und an dieser Stelle den Blick auf ihre mögliche Zukunft richten, dann liegt es nahe sich dabei von einem Blick auf eine Zukunft der Gesellschaft leiten zu lassen. Ein solcher Zugang wird hier angeleitet von einer soziologischen Perspektive (Luhmann 1998). Da auch diese Perspektive sich dem grundlegenden Dilemma der prinzipiellen Unzugänglichkeit von Zukunft nicht entziehen kann, sind und bleiben Überlegungen in diese Richtung Hypothesen, die mehr oder weniger gut plausibilisiert werden können. Eine dieser zentralen Vermutungen läuft darauf hinaus, dass sich unsere Gesellschaft an der Schwelle des Übergangs zu einer bereits 2001 vom Managementvordenker Peter Drucker angedachten „,next society“ (Drucker 2001) befindet, die sich mit der Überforderung durch die Einmischung vernetzt mitdenkender Computertechnologien auseinandersetzen und dabei zunehmend auf bislang gültige Ordnungsprinzipien (Zweck-Mittel-Relation, Hierarchie, Planung) verzichten muss. Die Überraschungsmomente, die durch global vernetzte Rechenkapazitäten und hochsensibel aufeinander reagierende Systemzustände für permanente Unruhe sorgen, unterlaufen zusammen mit der wachsenden Vielfalt möglicher Optionen bestehende Sicherheiten und erschüttert die tradierte Vorstellung eines in sich schlüssigen, fest verankerten und 
daher allen Legitimationsfragen entzogenen sinnstiftenden Handlungsrahmens. An seine Stelle rücken Ambivalenz und Uneindeutigkeit, die vom Zwang zur Entscheidung begleitet werden. „Wählen müssen“ lautet die Kurzformel für den Umgang mit der Paradoxie wachsender Ungewissheit bei zunehmender Optionsvielfalt - ohne dabei auf das Sicherungsnetz unverbrüchlicher (kultureller) Festlegungen zurückgreifen zu können, die als „unentscheidbare Entscheidungsprämissen“ (Niklas 2000: 222ff.) die individuellen Entscheidungslagen kollektiv zu entlasten wussten (Holzinger 2007). Kein Wunder, dass ausgerechnet von Künstlern - stets zuverlässigen Seismographen gesellschaftlichen Wandels - schon recht früh die Notwendigkeit eines neuen Instrumentariums im Umgang mit diesen Verwerfungen erkannt wurde. So plädiert etwa Robert Musil in seinem Roman „Der Mann ohne Eigenschaften“ für einen neuen Zugang zur Welt, den Möglichkeitssinn:

Wer ihn besitzt, sagt beispielsweise nicht: Hier ist dies oder das geschehen, wird geschehen, muss geschehen; sondern er erfindet: Hier könnte, sollte oder müsste geschehen; und wenn man ihm von irgend etwas erklärt, dass es so sei, wie es sei, dann denkt er: Nun, es könnte wahrscheinlich auch anders sein. So ließe sich der Möglichkeitssinn geradezu als die Fähigkeit definieren, alles, was ebenso gut sein könnte, zu denken und das, was ist, nicht wichtiger zu nehmen, als das, was nicht ist. (Musil 1978: 16, Orig. 1930)

\section{Stadt als gebaute Gesellschaft}

In dem Maße, wie eine Gesellschaft sich also neue Kulturtechniken einfallen lassen muss, um den eigenen Überforderungen durch Überschusssinn und Kontingenz zu begegnen, rückt unter anderem auch die Frage nach einem Ort für die Produktion und Konsumption dieser „Kulturgüter" in den Fokus der Aufmerksamkeit. Wir folgen dieser Aufmerksamkeitsverschiebung und gehen im zweiten Schritt der Frage nach, welche möglichen Auswirkungen die skizzierten Problemlagen einer „,next society“ im städtischen Kontext entfalten.

In seinem Aufsatz ,Stadtluft macht frei“ beschreibt Dirk Baecker in Anlehnung an Max Weber die Funktion der Stadt: „Die Funktion der Stadt besteht darin, das Miteinanderleben der Einwohner zu ermöglichen, obwohl, während und vielleicht sogar indem sie sich nicht kennen.“ (Baecker 2008: 3). Folgt man dieser Funktionsbestimmung, gewinnt der urbane Raum im Zusammenhang gesellschaftlicher Transformationen eine besondere Bedeutung. Gerade weil die Stadt seit jeher der Ort ist, an dem die Regeln des Miteinander nicht mehr der Berechenbar- 
keit und damit der Sicherheit persönlicher Bekanntschaft folgen, ist sie prädestiniert für Experimente, den Umgang mit Unbekanntem soweit zu balancieren, dass es aufregend genug bleibt, um Inspiration und Irritation bieten zu können, und gleichzeitig sicher und kontrollierbar genug, um nicht soviel Angst und Schrecken zu verbreiten, dass ihm nur noch mit Ausschluss oder Institutionalisierung begegnet werden muss. Für dieses Doppelspiel ist Stadt gleichzeitig Problem und Lösung. Die daran anschließende Frage aber lautete: Wie verändert sich die Form einer Stadt, damit sie unter veränderten gesellschaftlichen Strukturbedingungen ihre ureigenste Funktion aufrechterhalten kann?

In der modernen Gesellschaft ist es eine Stadt, die sich vor allen Dingen funktional gebärdet und im Plan zu ihrem Höhepunkt aufläuft. Im Plan wird festgehalten, was in der Stadt möglich und sinnvoll ist, er ist „blueprint“ für die Entwicklungsverläufe und konkreten Fassungen des Städtischen bis hin zu den Namen von Strassen und Plätzen, er regelt das Miteinander der Menschen genauso wie das der Verkehrsmittel und Flüsse. So zumindest die modernistische Utopie, auf einen Punkt gebracht etwa von Le Corbusier, der in seiner „Charta von Athen“ bereits 1941 die funktionalen Aspekte der Stadt in den Mittelpunkt stellte und damit das Denken über Stadt bis in die späten 1980er Jahre hinein domestizierte (Le Corbusier 1962; Hilpert 1984). Freilich bricht auch in die Utopie des Planbaren immer wieder die Ernüchterung ein, dass Geplantes wie auch Ungeplantes zwei Seiten einer Form sind. Erst durch den Plan werden die Abweichungen zu ihm sichtbar. Auch wenn dies in der Konsequenz zwar dazu führt, dass diese Abweichungen immer wieder lustvoller Anlass für weitere planerische Eingriffe sind, überwinden diese das grundlegende Dilemma jeglicher Planung nicht. In der ungeplanten Abweichung vom Plan reproduziert sich die moderne Stadt, entwickelt ihre Eigenarten und ihren Eigensinn zwischen Vielfalt und Norm.

\section{Die ,X-City“}

Wenn wir davon ausgehen, dass „X-Cities“ Orte sind, die sich beim Organisieren des Zusammenlebens von Unbekannten von der eigenen Undurchschaubarkeit leiten lassen, dann finden sich erste Hinweise auf ihre mögliche Form bei den Vordenkern einer auf Temporalität abzielenden Stadtentwicklung: dem situativen Urbanismus, der sich aus dem Gedankengut der Situationistischen Internationale, einer der letzten großen 
Bewegungen politisch motivierter Avantgarde, heraus entwickelt hat (Debord 1996; Haydn/Temel 2006; McDonough 2004). ${ }^{1}$

Weitere Hinweise dazu folgen den bereits angedeuteten soziologischen Diagnosen einer Gesellschaft, deren zukünftige Differenzierung im Zeichen des Leitmediums Computer darauf hinausläuft, dass die Entkopplung und Fragmentierung einzelner Sinn- und Lebenswelten zu einem Eigenleben jenseits einer verbindlichen Ordnungslogik führt. Nur im Bedarfsfall (der manchmal auch ein Zufall sein kann) wird ihr Zusammenspiel auf Zeit intensiviert, um es bei passender (und manchmal auch unpassender) Gelegenheit wieder aufs Spiel zu setzen. In der Strukturlogik von Netzwerken organisiert, entziehen sich die einzelnen Elemente einem übergeordneten Zusammenhang und reagieren einzig auf die wechselseitige Beobachtung von Kontrollversuchen, in deren Zusammenspiel - folgt man dem Netzwerktheoretiker Harrison C. White, letztendlich in einer Welt voller Überraschungen und Wahlzwängen, überhaupt erst ihre eigene Identität konstituiert wird (White 1992). Anstelle langfristiger Bindungen und überschaubarer Wissensbestände tritt die Ungewissheit des Projekts als einem auf Zeit hin abgestellten Arbeits- und Lebensumstand (Grabher 2002).

Für die X-City heißt dies, dass sie nur noch im Modus des Vorläufigen zu denken ist, bei der einzig die mitlaufende Beobachtung der eigenen Dekonstruktion Anlass gibt für weitere Anschlusshandlungen. Um mit der Vielfalt sich überlagernder (planerischer) Interventionen und überraschender Nebenwirkungen wie auch den unberechenbaren Einklumpungen an unvorsehbaren Orten umgehen zu können, muss diese Stadt undefinierte „Leerstellen“ bereithalten, um die eigene Überraschungsfähigkeit nicht aus den Augen zu verlieren. Im Windschatten aller Kontrollversuche des Öffentlichen (Levin/Frohne/Weibel 2002) wird sie darauf angewiesen sein, einen „space“ jenseits aller Deutungshoheiten offen zu halten, der als Container all die Ergebnisse rückkopplungsgefährdeter Handlungszusammenhänge (und welche Handlungszusammenhänge sind dies mittlerweile nicht?) zu beherbergen in der Lage ist.

Der Begriff, der von Dirk Baecker an dieser Stelle als Bezeichnung für diese Leerstellen einer X-City ins Feld geführt wird, erinnert an die anarchistischen Karnevalesken einer Umkehrung der Verhältnisse, wie sie vor allem vom Literaturtheoretiker Michail Bachtin (1987) für allerlei Faschingstreiben nachgezeichnet wurden, ohne allerdings die explizit politische Lesart einer Gegenkultur mitzuführen: Es sind Possen, die,

1 Für einen Überblick sorgt hier die Zeitschrift Arch+ in ihrer Ausgabe 183 vom Mai 2007. 
unterschiedlich motiviert, die Stadt und damit immer auch sich selbst mit dem Potenzial des Eingangs erwähnten Möglichkeitssinns versehen:

Im Anschluss an den Markt der tribalen Stadt, die Häuser der antiken Stadt und die Pläne der modernen Stadt recodieren die Possen die Leerstellen der Stadt. Diese sind jetzt nicht mehr nur Gelegenheiten zum Tausch, Orte des strategischen Kalküls im Umgang mit der Gesellschaft und überraschende Ergebnisse einer funktionalen Planung der Stadt, sondern sie sind Anlässe für Hausbesetzungen, Straßenfeste, terroristische Angriffe, Fischgründe für Sekten, Standorte unternehmerischer Neugründungen, Gegenstand künstlerischer Aktionen, Bühne für politische Auftritte, die sich allesamt nicht mehr an repräsentative Räume halten, sondern sich ihre location je nach Situation, Anlass und Absicht selber suchen. (Baecker 2008: 26)

Inmitten der ineinander getrennten Flows und Sphären (Sloterdijk 1998 bis 2004) greifen solcherart Possen zu beliebigen Zeiten die mal mehr, mal weniger frei verfügbaren Symbole und kulturellen Praktiken auf und programmieren sie um zu ubiquitären Zeichen einer Eigenlogik, die sich - frei von allen Sorgen um Anschlusskommunikation - im städtischen Raum entfalten und sich dabei und damit in etwas verwandeln, mit dem sie selbst nicht zu rechnen in der Lage sind. Die Ergebnisse, die dabei entstehen, sind keineswegs beschränkt auf die bis dato sich ins Spiel bringenden Ansprüche kreativer Klassen im Sinne Richard Floridas (2004), sondern umfassen alle lose gekoppelten „domains“, die das Netzwerk städtischer Identitäten ausmachen - inklusive der ständig mitrechnenden Computer, die aufgrund ihrer Geschwindigkeit, Vernetzungs- und Verarbeitungskapazität mit den zur Verfügung stehenden Daten für so manchen Überraschungserfolg im Sinne einer ganz eigenen Posse zu sorgen imstande sind.

Was dies für die Prozesse der Entwicklung und städtebaulichen Orchestrierung einer solchen Stadt bedeutet, soll uns anhand eines konkreten Beispiels im nachfolgenden Kapitel beschäftigen. Festhalten können wir, dass es sich mit hinreichender Sicherheit um ein „Spannungsspiel“ entlang einer der prominentesten architektonischen Formen handeln wird: dem Dach, seit jeher definiert als „Eingrenzung und Ausgrenzung im Interesse von Schutz, Orientierung und Versammlung“ (Baecker 2007: 73). So wie sich die Form des Dachs mit der Spannung auseinandersetzt, die eigene Innenseite (den Schutz) genauso attraktiv denken zu müssen wie die Außenseite (die Offenheit), um entsprechende Übergänge vom Orientierung stiftendem Bekannten zur Irritation auslösenden Überraschung und wieder zurück zu entwerfen, so muss die Stadt der Zukunft, noch viel radikaler als die der Gegenwart, mit den Widrigkei- 
ten „kausaler Komplexität, kommunikativer Überforderung und temporalem Eigensinn rechnen“ (Baecker 2007: 79). Wie diese Gratwanderung der permanenten Bezugnahme auf Unbestimmtes, das damit zugleich unter das Dach geholt wie auch auf Abstand gehalten werden muss, wie dieser Spagat zwischen der wärmenden Gemeinschaft des Bekannten und der coolen Fremde des Neuen aussehen kann, bleibt der Frage des Einzelfalls überlassen. Dass sie jedoch als Anforderung an die Möglichkeiten einer X-City immer wieder neu gestellt werden wird: davon kann man ausgehen (Baecker 2007).

\section{Alternative Stadtteil-Entwicklung: Das Projekt Graz-Reininghaus}

Ein ehemaliges Brauereigelände, grüne Felder, alter Bau- und Baumbestand, zwei malerische Teiche, mehrstöckige Lagerkeller, Gaststätte, Tennenmälzerei, Brunnenhaus und Gründervilla. 545.000 Quadratmeter Grund, 2 km westlich vom historischen Zentrum der zweitgrößten Stadt Österreichs gelegen: So präsentiert sich Graz-Reininghaus dem neugierigen Blick von außen. Hervorgegangen ist das Projekt aus dem Aufkauf sämtlicher Liegenschaften der Brau Union Österreich, die nach dem Verkauf an den Heineken Konzern im Zuge der daran üblicherweise anschließenden Portfoliobereinigung und Konzentration auf das Kerngeschäft ihren österreichweit gestreuten Besitz an Grund und Boden 2005 an einen privaten Immobilienentwickler, die Asset One AG, veräußert hat. Im Zuge der weiteren Verwertung der Liegenschaften wurde unmittelbar danach ein Großteil der 1,2 Millionen Quadratmeter weiterverkauft, um so den Finanzierungsbedarf für die Entwicklung dieses Stadtteils zu generieren.

Am Beginn des Entwicklungsprozesses von Graz-Reininghaus stand die Positionierung des Eigentümers, einen Stadtteil für ca. 12.000 Menschen nicht in der branchenüblichen Art und Weise zu konzipieren, sondern vielmehr einen experimentellen Zugang zu suchen, der jenseits aller kurzfristigen Renditeorientierung und optimierten Verwertungslogik mit genügend Zeit und Intelligenz aufwartet, um tatsächlich den Versuch wagen zu können, einen lebenswerten, von urbaner Vielfalt geprägtem „Stadtteil mit Eigenschaften“ entstehen zu lassen. Die Frage nach der Governance des Entstehungsprozesses, d. h. der Koordination und Steuerung aller beteiligten Akteure bis hin zum Zusammenleben der Bewohner des neuen Stadtteils stand dabei von Anfang an im Mittelpunkt der Überlegungen. Von vornherein war klar, dass es hierzu Abschied zu nehmen galt von den klassischen Vorstellungen einer durchgeplanten 
Vorgehensweise, die, mit der Kartierung des Bestandes beginnend, möglichst rasch zu einem Masterplan zu kommen hat, in dem sowohl der Ressourceneinsatz als auch die Planungssicherheiten bezüglich der einzubindenden Partner, namentlich der Stadtverwaltung sowie weiterer Investoren, schriftlich niedergelegt sind und der für eine scheinbar reibungslose „Abwicklung“ der Entwicklungs- und Investitionsbedarfe sorgt. In Abweichung vom Standard immobilientechnischer Gepflogenheiten wurde stattdessen ein Dialog mit unterschiedlichen nationalen wie internationalen Mit- und Vordenkern aus den Bereichen Stadtentwicklung, Kunst, Wissenschaft, Ökologie und Gesellschaft angestrebt, die eingeladen wurden, sich Gedanken zu einem wünschenswerten Stadtteil zu machen.

Um auch die Perspektive potenzieller Nutzer und Nutzerinnen im Blick zu behalten, wurde parallel dazu die ,werkstadt017“ gegründet, ein Forum von 32 Grazer Bürgern und Bürgerinnen, die ihre Überlegungen zu wichtigen Rahmenbedingungen und Eckpunkten eines neuen Stadtteils sammeln und im Rahmen einer Publikation veröffentlichen sollten. Entstanden ist daraus das Buch „Konzeptionen des Wünschenswerten - Was Städte über die Zukunft wissen sollten“, ein lebendiges Dokument gesellschaftspolitischer Reflexionen, wie Leben, Arbeit, Bildung und Urbanität in Zukunft aussehen könnten, und - in Rückbindung an das Potenzial eines gemeinsam gestalteten Entwicklungsprozesses über welche Eigenschaften ein Stadtteil verfügen sollte, um möglichst viele dieser Überlegungen beheimaten zu können.

Im Rahmen der weiteren Verdichtung entstand im Januar 2007 die „Struktur der Eigenschaften“, ein Netzwerk von Wertebündeln, mit der die zentralen Aspekte einer zukunftsfähigen Stadt zusammengefasst werden. Gerichtet an aktuelle und zukünftige Mitdenker und Umsetzer des Projekts, dient die Struktur der Eigenschaften als Instrument für die kollektive Reflexion und Leitfaden für die konkrete Gestaltung nachfolgender Planungs- und Baumaßnahmen.

Um den Diskurs mit den für die weitere Entwicklung des Stadtteils relevanten Gesprächspartnern zu intensivieren, wurde parallel dazu auch die „Reininghaus Gesellschaft“ gegründet: eine multidisziplinäre Diskussionsplattform, die sich öffentlich den Fragen einer lebenswerten urbanen Zukunft stellt und die jenseits aller formal-rechtlichen Aspekte als virtueller Akteur konzipiert wurde, der die Auseinandersetzung in und um den zukünftigen Stadtteil bereits in der Gegenwart vorwegnimmt. Mit einer Vielzahl von Veranstaltungen, Denksalons, Exkursionen und Publikationen wurde immer wieder dafür Sorge getragen, dass neue Impulse und bislang unbedachte Tatbestände in die Diskussion mit dem Eigentümer und weiteren relevanten Stakeholdern eingespeist wer- 
den, um damit die Idee eines permanent sich selbst beobachtenden, überraschungsfähigen Stadtteils bereits von Beginn an zu verankern.

Um den driftenden Entwicklungsprozess immer wieder neu zu fokussieren, wurden Ende 2007 vom Kernteam und dem Eigentümer fünf Standpunkte formuliert, die helfen sollten, der Dynamik einen inhaltlichen wie auch prozessualen Rahmen zu geben, mit dem die weitere Entwicklungsrichtung definiert und für alle Beteiligten ein hinreichend stabiler Orientierungsrahmen geschaffen wird. Die fünf Standpunkte legen in kondensierter Form fest, dass in Graz-Reininghaus ein eigenständiges Stadtzentrum von urbaner Vielfalt und Lebendigkeit mit einer unverwechselbaren Persönlichkeit entstehen soll, das in einem ergebnisoffenen und kooperativ geführten Prozess entwickelt wird und sowohl auf nachhaltigen wirtschaftlichen als auch städtebaulichen Erfolg ausgerichtet ist.

Auf dieser Grundlage wurde auch der nächste Schritt im Entwicklungsprozess gemeinsam festgelegt: Ab Mitte 2008 soll auf zwei miteinander verknüpften Handlungssträngen an einer robusten Form der Stadtentwicklung gearbeitet werden, mit der sowohl der Einstieg weiterer Entwicklungsunternehmer als auch das Zusammenspiel mit der Stadt Graz erleichtert werden soll. Während ein städtebaulich geprägter Zugang die unterschiedlichen Perspektiven in näheren Augenschein nimmt, die für die Realisierung des neuen Stadtteils im Sinne von „hard facts“ von zentraler Bedeutung sind, vertiefen stadtsoziologisch ausgerichtete Überlegungen die Frage nach den Bedingungen eines lebenswerten Miteinander im Spannungsfeld von irritierender Vielfalt und in sich ruhender Sicherheit und Orientierung.

Die Interdependenzen beider Dimensionen knüpfen nahtlos an die aktuellen Auseinandersetzungen in der Geographie, Stadtsoziologie und Kulturtheorie an: Wie müssen räumliche Gestaltungsmerkmale eines Ortes gedacht werden, der Menschen eine Heimat ermöglicht, die sich aus der Enge des Begriffs befreit hat und den bereits weiter oben skizzierten Parametern einer X-City nahekommt? (Maresch/Werber 2002; Schoer 2006; Löw 2001).

Während einerseits unter Beteiligung international anerkannter Architekturbüros und Fachexperten die Fragen nach Mobilität, Grünund Freiräumen, Energieversorgung, Wohnbau, Nutzungsvielfalt in unterschiedlichen Stadtszenarien bearbeitet werden, widmet sich die stadtsoziologische Fragestellung u. a. im Rahmen einer Forschungskooperation mit dem Lehrstuhl für Kulturtheorie und -analyse an der Zeppelin University Friedrichshafen der Frage nach den sozialen Gestaltungsdimensionen. Erklärtes Ziel beider Perspektiven ist die Verdichtung der gewonnenen Erkenntnisse in ein abstraktes Stadtmodell, das als 
erste Konkretisierung der durchgeführten Prozessschritte die unterschiedlichen Aspekte miteinander verknüpft und im Sinne einer Selbstfestlegung den Optionsraum für mögliche Planungsschritte weiter eingrenzt, ohne dabei sämtliche „Leerstellen“ für unerwartet auftauchende Gelegenheiten wie auch Possen im baeckerschen Sinne preiszugeben. Mit Hilfe dieses Modells des zukünftigen Stadtteils wird gleichsam eine Suchrichtung aufgespannt, die nicht zuletzt aufgrund ihrer Einbettung in die Prozessgeschichte das Risiko der Beliebigkeit eindämmt, ohne dem weiteren Entwicklungsprozess die Luft zum Atmen zu nehmen.

Ergänzt um vielfältige Experimente einer temporären Nutzung des Geländes sowie die laufende Abarbeitung städtebaulicher „Hausaufgaben“ (Veränderungen im Flächenwidmungsplan, Anbindung an die öffentliche Infrastruktur, Lobby-Arbeit in der Stadtverwaltung, Sanierung bestehender Industriedenkmäler usw.) fungiert das Stadtmodell als Attraktor für die Vielfalt möglicher Gestaltungsoptionen und ist Grundlage einer räumliche Teilung des Geländes in einzelne, autonom agierende Einheiten, die von unterschiedlichen Akteuren mit jeweils eigener Entwicklungslogik verwertet werden können. Das Zusammenspiel autonomer Partner, die sich durch Selbstbindung auf einen gemeinsamen Rahmen festlegen, der in der Lage ist, durch wenige, konsequent durchgehaltene Spielregeln eine größtmögliche Vielfalt hervorzubringen, wird durch ein Governance Modell abgesichert, welches die bereits skizzierten Überlegungen zur Steuerung einer X-City aufgreift und das nachhaltige Zusammenspiel benachbarter Projekte im Sinne heterarchischer, auf der Ökologie von Netzwerken aufbauender Koordinationsformen ermöglicht. Als iterativer Prozess einer fortlaufenden Selbsterneuerung konzipiert, kann die hier vorgestellte „Reininghaus Methode“ als Blaupause für einen Entwicklungsprozess jenseits des klassischen planerischen Vorgehens dienen: die Entstehung eines Stadtteils auf der Grundlage unklarer Rahmenbedingungen (Brand Eins 2008).

Sowohl die konsequent auf Überraschungsfähigkeit abgestellte Vorgehensweise als auch die gezielte Nutzung periodischer Auszeiten zur Reflexion des laufenden Geschehens, aber auch der Entscheidungsprozess für jeweilige Anschlussaktivitäten sind darauf ausgerichtet, unter permanentem Einbezug relevanter Schlüsselspieler einen Handlungsrahmen zu entwerfen, der ein evolutionäres Fortschreiten ermöglicht, ohne dabei mit einer (halbwegs) vollständigen Informationslage zu rechnen.

Es bleibt in der Tat über eine ungewöhnlich weite Strecke offen, wie der zukünftige Stadtteil, aber auch der weitergehende Prozess seiner Entwicklung aussehen wird - ein Umstand, der nicht zuletzt vom Eigentümer einiges an finanzieller wie auch politischer Potenz verlangt: Die 
Investitions- und Planungslogik möglicher Kooperationspartner stößt damit immer wieder auf einen Erwartungshorizont, der Zweifel und Enttäuschungen geradezu magisch anzieht. Nichts Schlimmeres gibt es für die Planungsstäbe einer Stadtverwaltung wie auch das Controlling zahlungswilliger Investoren, als in diesem Zusammenhang auf einen offenen Prozess verwiesen zu werden, der die Notwendigkeit der verlässlichen Kooperation nicht negiert und ihr gleichzeitig ständig den Boden unter den Füssen wegzieht. Der laufende Umgang mit dieser Zumutung gehört zu den anspruchsvollsten Herausforderungen der Gesamtprojektsteuerung.

\section{Zur Steuerungslogik von „X-Cities“}

Wir hatten bereits zu Beginn angedeutet, dass es sich bei der Frage nach der Beobachtung, Reflexion und Steuerung dieses Prozesses lohnen könnte, einen Blick auf Organisationen zu werfen, die sich bereits seit geraumer Zeit mit ähnlichen Fragestellungen beschäftigen. Welche Anleihen lassen sich aus den dort entwickelten Zugangsweisen und Werkzeugen nehmen, um die Frage nach einem zukunftsfähigen Umgang mit der eigenen Ungewissheit zu beantworten?

Erste Hinweise dazu werden sichtbar, wenn wir den Prozess der Strategieentwicklung näher betrachten, wie er vor allem aus einer systemtheoretischen, d. h. nicht normativen Perspektive heraus durchdekliniert wurde (Nagel/Wimmer 2002; Schreyögg 1999; Mintzberg 1999). In expliziter Abkehr von der Fülle präskriptiver Ansätze, in denen Strategiearbeit als expertenorientiertes Vorgehen vor allem dazu konzipiert wurde, einer Unternehmung die ,richtige“ Strategie vorzusetzen, wird mit dem ,Abschied vom Ideal der plandeterminierten Unternehmenssteuerung“ (Schreyögg 1999b: 389) ein Vorgehen eingeleitet, dass sich von den zentralen Prämissen eines traditionellen Organisationsverständnisses radikal verabschiedet.

Es ist auch hier die Einsicht in die Undurchschaubarkeit sowohl der eigenen Umwelten als auch der eigenen Zukunft, verbunden mit der Dekonstruktion der Vorstellung von Organisation als triviale Maschine, mit der die Hoffnung auf eine zweckrationale Planbarkeit der vielfältigen Aktivitäten einer Unternehmung bestenfalls als simple Kontrollfiktion bestehen kann. Wenn hingegen Strategiearbeit als zentraler Hebel für die Lernfähigkeit eines sozialen Systems konzipiert wird, welches sich vor dem Hintergrund des Nichtwissens von dem Anspruch an eine exakte Kalkulierbarkeit und damit Kontrollierbarkeit künftiger Entwicklungen verabschiedet, wird die Beschäftigung mit der eigenen Strategie zu 
einem kreativen Prozess der Selbsterneuerung, bei dem die eigene Identität im aufmerksamen Abgleich mit Umweltveränderungen und gewünschter, und damit entscheidbarer Selbstfestlegung immer wieder neu entworfen und in einem ko-evolutionären Prozess mit den relevanten Umwelten auf die eigene Überlebensfähigkeit hin überprüft und ggf. nachjustiert wird. Die dazu als Hilfestellung ausgelegten Werkzeuge etwa der Szenario-Entwicklung (Perspektive Zukunft), Kundennutzenoder Wettbewerbsanalyse (Perspektive Umwelt) oder StärkenSchwächen-Diagnose (Binnenperspektive) erleichtern durch die in ihnen angelegte Systematisierung die Denkarbeit, ersparen aber keinesfalls die notwendigen unternehmerischen Eingriffe, mit denen Organisationen erst zu ihrer Hochform auflaufen: die Umwandlung von Ungewissheit in Sicherheit durch Entscheidung (Lihman 2000; Krusche 2008). ${ }^{2}$

Die Parallelen zum weiter oben vorgestellten Projekt der Stadtentwicklung von Graz-Reininghaus liegen auf der Hand. Sowohl der Aspekt der Ungewissheit als auch die darin periodisch vorgenommenen Neuentwürfe der eigenen Identität vor dem Hintergrund sich wandelnder Marktgegebenheiten (aktuell: der Umgang mit der Krise des globalen Finanzsystems im Hinblick auf die Refinanzierungskosten weiterer Investitionen) und - viel entscheidender - unternehmerischer Festlegungen (im Sinne der Abwägung und Nutzung sich neu ergebender Chancen im blinden Fleck routinisierter Geschäftsgebaren) verweisen auf ein funktional äquivalentes Vorgehen. Die Selbstfestlegungen der Projektgruppe (inklusive natürlich des Eigentümers) entsprechen weitgehend den Entscheidungsprämissen eines Management-Teams, das mit Blick auf die Zukunft der eigenen Überlebenseinheit das Risiko einer Entscheidungsfindung auf sich nimmt. Auch wenn im Verlauf des Stadtentwicklungsprojekts die aus der Strategiearbeit zur Verfügung stehenden Werkzeuge nicht explizit zur Anwendung gebracht wurden, ist die Grundlogik des Vorgehens hier nicht zu übersehen.

Auch der Umgang mit Unerwartetem ist Bestandteil der laufenden Projektarbeit, wenn gleich auch hier die aus der Managementliteratur destillierten Handreichungen nicht oder zumindest nicht vollständig auf-

2 Ohne an dieser Stelle die vorgestellte Denkfigur weiter zu strapazieren: Durch die paradoxe Natur der Entscheidung gewinnt dieser Vorgang deutlich an Finesse. Da in jede Entscheidung unweigerlich ihre eigene Kontingenz eingeschrieben ist (man hätte ja auch jeweils anders entscheiden können), dekonstruiert jede Entscheidung ihre eigenen Prämissen und baut die Komplexität gleichsam als Bugwelle vor sich auf, die sie im Prozessieren wieder einkassiert. Die daraus resultierenden Legitimationsprobleme beschäftigen vor allem die moderne Führungstheorie. 
gegriffen und entsprechend adaptiert und umgesetzt wurden. Hier ist vor allem auf die aktuellen Arbeiten von Karl Weick und Kathleen Sutcliff $\mathrm{zu}$ verweisen, die sich funktionierende Hochleistungsorganisationen unter dem Aspekt der Sicherstellung der Leistungserbringung näher angeschaut haben (Weick/Sutcliffe 2001). Viele Aspekte der Quintessenz ihrer Forschung zu Organisationen (etwa Atomkraftwerken, aber auch Intensivstationen und Flugzeugträgern), die sich mit dem Risiko konsequenzenreicher Entscheidungen in einer zunehmend unberechenbaren und durch komplexe Wechselwirkungen gekennzeichneten Umwelt konfrontiert sehen, finden sich - wenn auch implizit - in der Konfiguration des Projekts Graz-Reininghaus wieder. Sei es die ,preoccupation with failure", die aufmerksame Beschäftigung mit Abweichungen und Fehlern, die Anlass für sofortige Neujustagen des gesamten Vorgehens sind, oder das „commitment to resilience“, mit dem durch eingespielte Abstimmungsmechanismen und einer guten Mischung von Expertenwissen und Improvisationskunst im Fall von Überraschungen zusätzliche Leistungsreserven mobilisiert werden können: Stets sind Flexibilität und Reaktionsvermögen wichtiger Bestandteil des laufenden Projektgeschehens, die vom gesamten Projektteam mit entsprechender Aufmerksamkeit über alle Konflikte und Meinungsverschiedenheiten hinaus gepflegt werden.

Die Weiterführung dieser Parallelität von Stadtentwicklung und Strategiearbeit gewinnt vor allem durch die Arbeiten des französischen Philosophen und Ostasienkenners François Jullien (1999, 2006; sowie Baecker 1994) eine deutliche Erweiterung, die ebenfalls wertvolle Hinweise auf die Governance Principles von X-Cities liefert. In seinen Ausführungen zum traditionellen chinesischen Strategieverständnis kontrastiert Jullien die Grundlagen westlicher Modellbildung (mit der daran anschließenden Zielbildung und Planerstellung) mit einer Vorstellung von Wirksamkeit, die sich aus dem Situationspotenzial eines Sachverhalts erschließt und - insbesondere in Situationen, die durch hohe Unbestimmtheit und Vielfalt der Optionen, sprich Kontingenz geprägt sind eher auf ein Gewähren lassen der in der konkreten Situation eingeschriebenen Dynamik denn auf einen mit heroischer Geste produzierten (gezielten) Eingriff in den Lauf der Dinge hinausläuft. Mit der genauen Lesart wird deutlich, dass es sich hierbei in der Sache weder um ein passives sich treiben lassen noch im Vergleich um die geläufigen Verkürzungen eines esoterisch aufgeladenen abendländischen Blicks auf morgenländische Traditionen handeln: Eingewoben in ein hoch komplexes Netz kausaler Wechselbeziehungen sucht der solcherart geschulte Beobachter permanent nach Möglichkeiten, eine konkrete Situation produktiv werden zu lassen. Durch minimale Interventionen, und mit optimalem 
timing (das weniger glückliches Zusammentreffen von günstigen Umständen denn kluges Abwarten der größtmöglichen Potenzialität ist), werden die Kontextbedingungen verändert, die einer Situation ermöglichen, sich zu entfalten. Dies ist weder passives Abwarten noch aktives Handeln. Sondern Tun durch Nicht-Tun, um es mit Hilfe einer paradoxen Wendung auszudrücken. Wirksamkeit ist in dieser Lesart immer das Resultat eines Prozesses, ganz und gar verankert in der Empirie der Verhältnisse und damit hoch kontextabhängig. Strategisches Handeln besteht dann in erster Linie darin, das Potenzial einer Situation zu erfassen, noch bevor sie in einer Handlung kulminiert, und - im Sinne einer ,vorausschauenden Selbsterneuerung“ (Wimmer 2000) - dann, wenn es (noch) leicht fällt, so im Sinne der eigenen Interessen zu intervenieren, dass gleichsam eine schiefe Ebene entsteht, auf der Dinge wie von selbst ins Rollen geraten und man sie nur noch in Empfang zu nehmen braucht, wenn es soweit ist. Diese Form der Leichtigkeit und Effizienz (Jullien 2006: 84ff.) ist die hohe Kunst strategischen Handelns.

Es lohnt, genau an dieser Stelle auch auf die überraschenden Querverbindungen und Ergänzungen hinzuweisen, die sich bei der Frage nach wirksamen Steuerungsprinzipien für X-Cities ergeben, wenn eine Umstellung der inneren Disposition von Planung auf Improvisation erfolgt. Die aus der Jazz-Musik und dem modernen Tanztheater kondensierte Form der Improvisation (Berliner 1994; Johnstone 1979) lebt in einem überaus hohen Maße aus dem Situationspotenzial einer Performance.

Improvisation involves reworking precomposed material and designs in relation to unanticipated ideas conceived, shaped, and transformed under the special conditions of performance, thereby adding unique features to every creation. (Berliner, 1994: 241)

Einfache Regeln für komplexe Spiele, so könnte man die Grundlogik eines improvisierenden Vorgehens beschreiben. Ebenfalls tief verankert in der Empirie des konkreten Sachverhalts, baut Improvisation immer auf Bestehendem auf, das Material ist für vielfältige rekursive Verknüpfungen, Variationen und Transformationen. In den Worten des großen Altmeisters des Jazz, Charles Mingus: ,You can't improvise on nothing; you've gotta improvise on something.“ (Kernfeld 1995: 119)

Ihr prozesshafter Charakter ist in erster Linie auf Anschlusskommunikation gerichtet, weniger auf das Ergebnis des Handelns (und schon gar nicht auf die Bewertung des Ergebnisses im Sinne moralischer Kategorien) - das Resultat ist gleichsam das Nebenergebnis des aufmerksamen passiv-aktiven Spielens (listen to get into it!) mit dem vorgefunde- 
nen Material, sei es die Melodie und die anwesenden Mitmusiker im Jazz oder die bis dato vorhandene Story und die darin mitspielenden Schauspieler beim Improvisationstheater.

Eingespannt in das bereits erwähnte Netzwerk von Identität und Kontrolle, entwickeln sich in der Geborgenheit einfacher Spielregeln (,Just Say Yes“) überraschende Wendungen und Volten, die der Vielfalt nachbarschaftlich verbundener Projekte gerechter werden als komplizierte Vorgaben besorgter Spielmacher. Nicht nur für die Beobachtung und das Management von Organisationen (Weick 1998; Dell 2002), sondern gerade auch für die Gestaltung von urbanen Settings kann das Spiel mit dem Unbestimmten zu einer lustvollen Prämisse einer ,ungoverned governance“ werden, bei der es in erster Linie darum geht,

sich der jeweiligen Referenzsysteme bewusst zu werden und Räume der Information, Umwelten des Organismus, Milieus der Gesellschaft und Faszination des Bewusstseins weniger zu fusionieren als vielmehr zu unterscheiden und in ihrem jeweiligen Eigenwert aufeinander zu beziehen. (Baecker 2007: 79)

All die hier aufgeführten Steuerungsprinzipien bilden gleichsam das unausgesprochene Rückrat des Stadtentwicklungsprozesses in GrazReininghaus, der explizit als ergebnisoffener und auf Nachhaltigkeit ausgerichteter, hoch reflexiver Entstehungsprozess angelegt ist, der zu immer neuen Variationen und Verästelungen geführt hat, die stets weniger geplant als auf die Ausschöpfung der jeweiligen Situationspotenziale hin ausgerichtet waren. Das Suchraster, das dadurch aufgespannt wurde, hat zu einer hohen Komplexität und Vielfalt der in den Blick genommenen Sachverhalte geführt, die im Projektteam schon allein aus Gründen der Praktikabilität immer wieder auf ein handhabbares Maß zurechtgestutzt werden mussten.

Diese Verdichtungsphasen sind als notwendige Punkte eines Innehaltens explizit Bestandteil des Entwicklungsprozesses und tragen immer mit Blick auf das aktuell zur Verfügung stehende Situationspotenzial, dass je nach Umstand von überraschenden Koalitionen mit der Stadt bis hin zu Teilverkäufen des Geländes gehen kann - bereits den Kern weiterführender Aktivitäten in sich. Exemplarisch spiegeln die hier skizzierten abstrakten Prinzipien einer Governance-Struktur die Knotenpunkte einer Stadtentwicklung, die als „unendliches Spiel“ (Carse 1986) ${ }^{3}$ angelegt ist und die durch die Praxis der mitlaufenden Selbstver-

3 Im Unterschied zu endlichen Spielen spielt man unendliche Spiele nicht um sie zu gewinnen, sondern um sie zu spielen. 
änderung nicht mit der Fertigstellung von Plätzen und Gebäuden abgeschlossen sein kann - ganz im Sinne einer X-City, die nie ist, sondern immer nur wird. Die Frage, inwieweit die Stadtteilentwicklung in GrazReininghaus mitsamt des dort verfolgten methodischen Vorgehens - der Reininghaus Methode - dafür ein erfolgreiches Exempel statuiert, muss daher an dieser Stelle nicht zuletzt aufgrund der Ergebnisoffenheit des Prozesses tatsächlich unbeantwortet bleiben. Hier gilt nach wie vor der alte Satz des Großmeisters entspannter Gegenwarten, Bob Marley: „Only time will tell..."

\section{Literatur}

Bachtin, Michail (1987): Rabelais und seine Welt. Volkskultur als Gegenkultur, Frankfurt/Main: Suhrkamp.

Baecker, Dirk (1994): Postheroisches Management: Ein Vademecum, Berlin: Merve Verlag.

Baecker, Dirk (2004): „Miteinander leben, ohne sich zu kennen: Die Ökologie der Stadt“". Soziale Systeme 10, S. 257-272.

Baecker, Dirk (2007): Studien zur nächsten Gesellschaft, Frankfurt/Main: Suhrkamp.

Baecker, Dirk (2008): „Stadtluft macht frei“, bisher unveröffentlichtes Manuskript.

Berliner, Paul (1994): Thinking in Jazz: The Infinite Art of Improvisation, Chicago: University of Chicago Press.

Brand Eins (2008): „Ein Ort für die Zukunft“. Brand Eins, Ausgabe 8, S. 123-129.

Carse, James P. (1986): Endliche und unendliche Spiele, Stuttgart: Klett-Cotta.

Debord, Guy (1996): Die Gesellschaft des Spektakels, Berlin: Bittermann.

Dell, Christopher (2002): Prinzip Improvisation, Köln: Walther König.

Drucker, Peter (2001): „The Next Society: A Survey of the Near Future“. In: The Economist, 3.11.2001.

Florida, Richard (2004): The Rise of the Creative Class. And How It's Transforming Work, Leisure, Community and Everyday Life, New York: Basic Books.

Grabher, Gernot (2002): „The Project Ecology of Advertising: Talents, Tasks, and Teams“. In: Regional Studies, Special Issue, Vol. 36 (5), 245-262.

Haydn, Florian/Temel, Robert (Hg.) (2006): Temporäre Räume, Basel: Birkhäuser Verlag. 
Hilpert, Thilo (Hg.) (1984): „Le Corbusiers ,Charta von Athen“. Texte und Dokumente. Kritische Neuausgabe“. Bauwelt Fundamente 56. Braunschweig, Wiesbaden: Vieweg.

Holzinger, Markus (2007): Kontingenz in der Gegenwartsgesellschaft Dimensionen eines Leitbegriffs moderner Sozialtheorie, Bielefeld: transcript Verlag.

Johnstone, Keith (1979): Impro: Improvisation and the Theatre, New York: Theatre Arts Book.

Jullien, François (1999): Über die Wirksamkeit, Berlin: Merve Verlag.

Jullien, François (2006): Vortrag vor Managern über Wirksamkeit und Effizienz in China und im Westen, Berlin: Merve Verlag.

Kernfeld, Barry (1995): Die Enzyklopädie des Jazz: Die Geschichte des Jazz im Spiegel der wichtigsten Aufnahmen, Bern: Scherz.

Krusche, Bernhard (2008): Paradoxien der Führung, Heidelberg: CarlAuer-Systeme.

Koolhaas, Rem/Mau, Bruce/Sigler, Jennifer (1998): S, M X, XL. New York: Monacelli Press.

Le Corbusier (1962): An die Studenten - Die Charte d'Athènes, Hamburg: Rowohlt.

Levin, Thomas Y./Frohne, Ursula/Weibel, Peter (2002): CTRL Space. Rhetorics of Surveillance from Bentham to Big Brother, ZKM Publikation, Karlsruhe.

Löw, Martina (2001): Raumsoziologie, Frankfurt/Main: Suhrkamp.

Luhmann, Niklas (1998): Die Gesellschaft der Gesellschaft, Frankfurt/Main: Suhrkamp.

Luhmann, Niklas (2000): Organisation und Entscheidung, Opladen: Westdeutscher Verlag.

Maresch, Rudolf/Werber, Niels (2002), In: Maresch, Rolf/Werber, Niels (Hg.): Raum, Wissen, Macht, Frankfurt/Main: Suhrkamp, S. 7-30.

McDonough, Thomas (Hg.) (2004): Guy Debord and the Situationist International, Cambridge: Cambridge University Press.

Mintzberg, Henry (1999): Strategy Safari, Wien: Ueberreuter.

Musil, Robert (1978, Orig. 1930): Der Mann ohne Eigenschaften. Reinbek bei Hamburg: Rowohlt, Band 1.

Nagel, Reinhardt/Wimmer, Rudolf (2002): Systemische Strategieentwicklung, Stuttgart: Schäffer-Poesche.

Peters, Tom (2003): „Just Say Yes“. In: Bennis, Warren/Spreitzer, Gretchen/Cummings, Thomas (Hg.): The Future of Leadership, San Fransisco: Jossey-Bass, S. 177-188.

Schreyögg, Georg (1999b): „Strategisches Management - Entwicklungstendenzen und Zukunftsperspektiven“. Die Unternehmung - 
Schweizerische Zeitschrift für betriebswirtschaftliche Forschung und Praxis, 53 (1999), S. 387-407

Schroer, Markus (2006): Räume, Orte, Grenzen, Frankfurt/Main: Suhrkamp.

Sloterdijk, Peter: Sphären I - III, Frankfurt/Main: Suhrkamp.

Weick, Karin (1998): „Improvisation as a Mindset for Organizational Analysis”. Organization Science, Vol. 9, No. 5.

Weick, Karin/Sutcliffe, Kathleen (2001): Managing the Unexpected. Assuring High Performance in an Age of Complexity, San Francisco: Jossey-Bass.

White, Harrison C. (1992): Identity and Control, Princeton: Princeton University Press.

Wimmer, Rudolf (2000): „Wie lernfähig sind Organisationen? Zur Problematik einer vorausschauenden Selbsterneuerung sozialer Systeme“. In: Hejl, Peter M./Stahl, Heinz (Hg.): Management und Wirklichkeit, Heidelberg: Carl-Auer-Systeme, S. 265-293. 
FAZIT 



\section{Diagnosen, Handlungsoptionen sowie zehn abschließende Thesen zur Governance der Kreativwirtschaft}

BASTIAN LANGE, ARES KALANDIDES, BIRgIT StÖBER, INGA WELLMANN

Im Jahr 2007 begannen wir mit der Konzeption der vorliegenden Anthologie. Der damalige Kontext war durch generelles wirtschaftliches Wachstum bestimmt, mehrheitlich getragen durch den weiteren Ausbau der traditionell starken Exportbranchen: Mit einer stabilen Automobilindustrie, so die damalige Einschätzung und wirtschaftspolitische Hoffnung, können eine bundesrepublikanische und US-amerikanische Wirtschaft nachhaltig in bekannten Bahnen das Wohl des volkswirtschaftlichen Einkommens bestimmen. Allerorten dominierte das Bild des heroischen Unternehmers, der - im schumpeterschen Sinn - als kreativer Zerstörer immer in der Lage war, pionierartig Neues zu erschaffen. Diese Figur bestimmte auch das Bild des Unternehmers, der meinte, den Unwegsamkeiten einer immer ruppigeren Globalisierung zu trotzen.

Gleichzeitig nahm die Akzeptanz des Branchenkonglomerats Kreativwirtschaft aufgrund der vorgelegten Wachstumszahlen und ihrer Rolle als Aushängeschild der Städte stetig zu. Dabei verblieb die Kreativwirtschaft aber noch weitestgehend in der Position des Exoten: Die Flüchtigkeit und Wandlungsfähigkeit erschwerten zudem einen systematischen und zugleich fördernden Zugriff auf dieses Handlungsfeld. Kurzum: Die Bekanntheit des Phänomens Kreativwirtschaft ging nicht mit einem Zugewinn an Steuerungskompetenz einher.

Während wir die letzten Beiträge dieser Anthologie sammeln und bearbeiten, bestimmt die globale Finanzmarkt-, Automobil- und Immobilienkrise den Kontext der Druckvorbereitung. Unerwartet erhalten dadurch viele Perspektiven dieser Anthologie eine gestiegene Relevanz. 
Viele Teilbranchen der Kreativwirtschaft sind möglicherweise durch ihre Kleinteiligkeit, ihr erprobtes Krisenmanagement, ihre schmale und realwirtschaftliche Kapitaldeckung sowie ihre einfache und pragmatische technologische Basis besser gegen die Unwuchten der wachsenden Wirtschaftskrise gefeit, als dies bspw. technologie- und finanzintensive Branchen sind. Einige Teilbranchen der Kreativwirtschaft, wie z. B. die Bereiche Film und Architektur, sind zweifelsohne hart von der Krise betroffen.

Doch nicht nur die betriebswirtschaftlichen Basisparameter sprechen paradoxerweise für eine neue Kultur des ,weniger ist mehr“ vieler Teilbranchen der Kreativwirtschaft, die in selbstverantwortlichen Kollektiven und Netzwerken organisiert sind. Wir reden damit nicht dem Credo „,arm aber glücklich“ das Wort, ebenso wie wir wenig Gefallen an prekären Arbeitsverhältnissen finden. Doch das Scheitern der Massenproduktion durch den Konzernkapitalismus geht einher mit dem „Ende des heroischen Unternehmers“ (Misik 2009).

Dadurch eröffnen sich Fragen, wie im Nachgang dieser desaströsen Ära des Turbokapitalismus ein wirtschaftlicher, gesellschaftlicher und sinnstiftender Neuanfang möglich ist. Dieser Wandel bedarf - im keynesschen Sinne - eines starken Staates, der deutlicher als bisher die Leistung der Kreativwirtschaft honoriert. Bei der Suche nach Lösungen müsste sich der Wandel weg von der Ära des zentralistischen Staatsverständnisses hin zu einem netzwerkartigen, aktivierenden Staat vollends durchsetzen: Als Steuerungsmodell eröffnet sich notwendigerweise im Nachgang des heroischen Unternehmertums der Weg für ein postheroisches Management. Dabei könnte die Organisationspraxis der Kreativwirtschaft eine Quelle der Inspiration sein, da im Gewand des Branchenkonglomerats Kreativwirtschaft ein neuer, zweifelsohne vielschichtiger Modus der Ökonomie aufkeimt, in dem sich grundsätzlich andere Werte, Verfahrensweisen und wirtschaftliche Strategien zu erkennen geben. Diese skizzieren wir hier im Folgenden als Steuerungs- und Organisationspraxis vorwiegend junger emergierender Märkte, wie in Teilen ebenso traditioneller handwerklichorientierter Manufakturstrukturen in einem globalisierten und digitalisierten Kontext.

Arbeit in Netzwerken und Schwärmen - Die Kreativwirtschaft folgt einer Netzwerklogik, die sich auch aus den sozio-technologischen Entwicklungen einer digitalisierten Wissensgesellschaft ergibt. Neue, meist informelle Vergemeinschaftungsformen, die kollektive Ressourcen bündeln, zugleich aber auch die Autonomie und Individualität des Einzelnen gewährleisten, prägen die Arbeitspraxis.

Unvollständige Akteure - Die meisten Kreativarbeiter definieren sich über den Bezug zu anderen, d. h. sie entfalten ihr Potenzial erst in 
der Verbindung zu Netzwerkpartnern. Der relationale Charakter ist den kreativen Ökonomien als Wesensmerkmal eingeschrieben, auch in Hinblick auf die Formierung von Märkten und Produkten. Lebensweltliche Interaktionsforen (Co-working Spaces) in Kombination mit PeeringModellen (kostenneutraler Datenaustausch über Internet Protocol (IP)Adressen) gewinnen an Bedeutung.

Schnittstellenkompetenz - Die Kreativwirtschaft verortet sich sektorenübergreifend und zeichnet sich durch hohe Schnittstellenorientierung aus. Kreative Akteure arbeiten wechselseitig an den Rändern etablierter Disziplinen und entwickeln hybride, transdisziplinäre Tätigkeitsprofile, um selbstbestimmt mit der Komplexität und Dynamik kreativer Ökonomien umzugehen.

Nischenorientierung und Spezialistentum - Geringe Transaktionskosten durch moderne Informationstechnologien ermöglichen es dem Einzelnen, sich mit seinen Ideen ohne allzu großes Risiko auf dem Markt auszuprobieren und Nischen zu besetzen. Authentizität und Differenz gelten als wichtige Alleinstellungsmerkmale in einer Ökonomie, die u. a. auf der Grundlage von Anerkennung des Neuen funktioniert und dabei immer auf der Suche nach dem Randständigen wie gleichsam Unerwartetem ist.

Sozialer und kultureller Mehrwert - Immaterielle Wertschöpfung, die sich aus der sozialen und kulturellen Sphäre speist, kennzeichnet die kreative Ökonomie und bildet damit eine deutlich wertorientierte und von Nachhaltigkeit geprägte Haltung zu wirtschaftlichem Handeln in einer globalisierten Gesellschaft.

Strategien der Bricolage und des kulturellen Hackings - Im Umgang mit einem von Unsicherheit und ständigem Wandel geprägten wirtschaftlichen Kontext entwickeln die Akteure situative, von Improvisation und Intervention geprägte Strategien, die ihnen Selbstermächtigung ermöglichen und bestehende Ordnungen laufend in Frage stellen. Dadurch ergeben sich auch neue, sich den jeweiligen Erfordernissen ständig anpassende Formen der (Selbst-)Kontrolle.

Zeitalter der Konnektivität und Kooperation - Die auf Repräsentation und Hierarchie abgestellten Strukturen des Industriezeitalters werden in den kreativen Ökonomien durch kooperative, heterarchische, also dezentrale und im Bezug auf Machtverhältnisse ausgeglichene Handlungsansätze und Organisationszusammenhänge abgelöst, die - im Sinne von „,co-opetition“ - trotzdem wettbewerbsorientiert sind.

Raumrelevanz - Städte werden als Schnitt- und Berührungsstellen verschiedener sich kreuzender Netzwerkdimensionen für die Kreativwirtschaft bedeutsam. In dem Maße, wie die Ausrichtung auf Metropolregionen wächst, eröffnen sich aber auch für vermeintlich entschleunigte 
Räume abseits des Buzz vielfältige Chancen der Profilierung durch Kreativwirtschaft.

Daraus ergeben sich drei abschließende Diagnosen:

\section{Self-Governance der Kreativwirtschaft}

Die Kreativwirtschaft setzt sich aus spezifischen Strukturen und Logiken mit überaus heterogenen Repräsentanten zusammen, seien es Global Player in der Medienbranche oder Mikrounternehmer in der Designbranche. Allgemein verbindliche Steuerungslösungen für die gesamte Kreativwirtschaft sind daher wenig adäquat. Denn abgesehen von den relativ wenigen Stars der Kreativwirtschaft, lassen sich bei Betrachtung der meisten Arbeitsbiographien häufig stark risikobehaftete Lebensumstände und Phänomene erkennen. Die auffallende Kleinteiligkeit und Heterogenität der Kreativwirtschaft und ihrer Akteure basiert auf einem hohen Maß an Selbststeuerungsfähigkeit und gilt weniger als heroischer Behauptungsanspruch, denn als berufsbiographischer Entwurf 2.0, der sich (notwendigerweise) abseits etablierter Angestelltenkulturen eröffnet.

Von Außen mit einem Steuerungsanspruch an diese Mikrokollektive heranzutreten, ist somit ein Steuerungsparadoxon: Da sich die Produktionspraxis in einem vielschichtigem Kontinuum vollzieht, kann nur durch „Teilnahme“ an der Binnensicht steuernd eingegriffen werden. Denn erst wenn die marktwirtschaftlichen, intermediären und fachadministrativen Akteure der Kreativwirtschaft den Eindruck erhalten, dass sie gesteuert werden sollen, können daraus situative und passgenaue Steuerungen erwachsen. Getreu der Erkenntnis, dass man Kontrolle nur ausüben kann, wenn man selbst kontrolliert wird, erscheint es somit ratsam, die Berührungs- und Schnittstelle verschiedener Governance-Arrangements in den Blick zu nehmen. An diesen Sollbruchstellen unterschiedlicher Governance-Ansprüche erwachsen Lösungen einer gelingenden Eingriffs- und Gestaltungspraxis. Diese haben dann aber zunehmend eher den Charakter von Interventionen als von linearen wirtschaftspolitischen Zielrichtlinien.

\section{Co-Governance der Kreativwirtschaft}

Die Kreativwirtschaft ist nicht nur als ein wirtschaftliches Wachstumsfeld anzusprechen, wie dies unlängst das Forschungsgutachten zu den gesamtwirtschaftlichen Perspektiven der Kultur- und Kreativwirtschaft in Deutschland im Jahr 2009 ermittelt hat. Sie ist auch als ein innovatives Reservoir vielfältigster organisatorischer und institutioneller Antworten auf den Niedergang des überhitzten Spätkapitalismus mit zu 
großen und unflexiblen Einheiten anzusprechen. Als solcher gebührt der Kreativwirtschaft ein prominenter Platz bei der Suche nach adäquaten Steuerungsoptionen für eine Ökonomie, die sich in kleinen Schwärmen und Produktionsnischen dem Zentralismus politischer und wirtschaftlicher Repräsentanz erwehrt. Aus diesem Blickwinkel bedarf es eines Um- und Neudenkens von Haltungen und Ansätzen in Bezug auf Steuerung. Diese Haltungen fassen wir als postheroisches Management von Produktion und den dafür notwendigen sozialen und kulturellen Interaktionen auf (Baecker 1994). Wenn somit die intermediäre Ebene zwischen Individuum und Gesellschaft aufgewertet wird, wenn also kreative Tätigkeiten in flexiblen informellen Netzwerken relevanter werden, dann leiten sich daraus Fragen zur Bewältigung von Leadership in strukturell instabilen Situationen ab. Leadership in wachsenden Krisenzeiten mit schrumpfenden Etats eröffnet sich erst durch die Akzeptanz einer im Grunde genommen paradoxen Basis: Da sich Self-Governance von ihrem schwarmartigen Initialstadium zu professionalisierten Plattformen entwickelt hat, muss die Frage nach Leitung - wenn man sie als zeitgemäß erachtet - neu gestellt werden.

Der Fokus auf weitestgehend unabhängige und selbststeuernde Produktionsnetze erscheint uns aber zu einseitig, weshalb wir ebenso in etablierten politischen, wirtschaftlichen und kulturellen Bereichen für ein neues Selbstverständnis von Förderung der Kreativwirtschaft plädieren.

\section{Hierarchische Governance der Kreativwirtschaft}

Auch wenn die Kreativwirtschaft nur schwer mit klassischen Topdown Steuerungen im Sinne der hierarchischen Governance in Verbindung zu bringen ist, so ist dieses Prinzip nach wie vor präsent: Zum Beispiel auf der Ebene „offizieller“ Definitionen des Branchenkonstrukts Kreativwirtschaft, bei der Vergabe von finanzieller Förderung für die Filmbranche oder bei der Außendarstellung einer Nation durch das Thema Kreativwirtschaft im Ausland. Die vorliegende Anthologie beinhaltet verschiedene Beispiele dieser Top-down Steuerungen, sei es die sehr offensichtliche Art aus Singapur oder auch die subtilere Arten wie sie dem Beispiel von Berlin oder dem Ruhrgebiet innewohnen. Bei all diesen Aktivitäten vollzieht sich eine Instrumentalisierung von Kunst und Kultur, von der einzelne kreative Branchen oder Akteure jedoch profitieren können, z. B. durch neu geschaffene Absatzmärkte und die mitunter größere Einflussnahme in den sich daraus ergebenen neuen Governancestrukturen. Wir schließen die vorliegende Anthologie mit zehn handlungsorientierten Thesen, von denen wir uns weitere fruchtbare Denkanstösse und Diskussionen erhoffen: 


\section{Thesen zur Governance der Kreativwirtschaft}

1. Förderansätze im Feld der Kreativwirtschaft müssen immer vom Paradoxon des kreativen Imperativs - „sei kreativ!“ - ausgehen. Eine Kontextsteuerung an Stelle von direktionaler Einflussnahme steht an.

2. Das hohe Maß an risikobehafteter Selbststeuerung bedarf ebenso einer offiziellen Politik der Anerkennung wie einer intelligenten Kooperation etablierter Institutionen mit den freien Netzwerken der Kreativen. Dies bedeutet auch, dass es in der Politik einer Offenheit gegenüber neuer Formen der Meinungsartikulation bedarf.

3. Eine Politik der Förderung kann sich auch durch Praktiken der temporären Nichtbeachtung, des kurzzeitigen Weghörens, des bewussten Wegschauens äußern, um eigenlogische Ereignisse nicht im Keim zu ersticken. Steuerung kann durch gewollte Nicht-Steuerung erfolgen. Es muss wesentlich mehr Unschärfe zugelassen werden.

4. Will die öffentliche Hand Kreativwirtschaft durch wirtschafts- und kulturpolitische Steuerungen fördern, transformiert sich ihre bisherige alimentierende Daseinsfunktion zu der einer aktivierenden Trägerschaft. Sie verbindet vorhandene Potenziale und bündelt Kräfte, anstatt dass sie sich einzig über finanzielle Mittelvergabe definiert.

5. Es gilt, die staatliche Administration in der Form zu stärken, dass sie mit einem erweiterten Bestand an ausgewiesenen Fachkräften - ,intelligent agents“ - zunehmend versierter und proaktiver der Heterogenität der Kreativwirtschaft begegnen kann.

6. Kreativwirtschaftliche Praktiken führen zu einer Neubewertung bisher bekannter und gewohnter räumlicher, thematischer und professionsspezifischer Maßstabs- und Interaktionsebenen. Somit wird die Notwendigkeit, neue Governanceformen zu eröffnen größer denn je, wenngleich paradoxerweise - die Steuerungswirkungen aufgrund unsicherer Rahmenbedingungen vermutlich eher ab- als zunehmen werden.

7. Kreativwirtschaftliche Selbstorganisation konterkariert die Rhetorik des Leadership sowie des Urban Leaders. Traditionellen Leadern fehlt zunehmend die soziale Gefolgschaft, postmoderne Trickster können aber Gefolgschaften temporär hinter sich vereinen. Der Trickster ist als Kulturheros ein Stifter von Kultur und ein Medium kultureller Veränderung, dem kreative Problemlösungen gelingen, indem die Definition und 
die Funktion des gerade zur Hand liegenden überschritten wird und die zur Verfügung stehenden Mittel erfinderisch umgedeutet und dann genutzt werden.

8. Kontrolle über Steuerungsverfahren erwächst nur, wenn sich Governance-Subjekte kontrollieren und steuern lassen (wollen). Dies setzt eine Bereitschaft aller Beteiligten voraus, miteinander in Dialog zu treten und zeitgemäße Austausch- und Begegnungsformate zu entwickeln. Dies muss im Falle der Kreativwirtschaft über die klassischen Strukturen politischer Einflussnahme hinausgehen, da aufgrund der Heterogenität und Dynamik der Kreativwirtschaft eine traditionell organisierte Interessenvertretung nicht realisierbar ist.

9. Die Stadt ist gleichsam der relevante Ort für die sozialen Interaktionen der Marktteilnehmer und gleichzeitig stellen diese rückwirkend durch ihre Kommunikationspraxis neue und somit differente Orte herstellen. Dadurch wird sich Stadtentwicklungspolitik immer dezidierter mit der Kreativwirtschaft auseinandersetzen müssen.

10. Die Figur des Schnittstellenakteurs gewinnt in seiner Rolle als gesellschaftlicher Mittler an Relevanz. Es bedarf einer Professionalisierung kultureller Intermediäre, die sich souverän zwischen den verschiedenen Funktionssystemen bewegen und in der Lage sind, zwischen alten (industriekapitalistischen) und neuen (netzwerkökonomischen) Denk- und Handlungsweisen zu vermitteln. Dies können kulturwirtschaftliche Einzelakteure sein, ebenso wie staatlich unterstützte intermediäre Institutionen.

\section{Literatur}

Baecker, Dirk (1994): Postheroisches Management: Ein Vademecum, Berlin: Merve-Verlag.

Misik, Robert (2009): Das Ende des heroischen Unternehmers. In: Blätter für deutsche und internationale Politik 2, S. 61-70. 



\section{Autorinnen und Autoren}

Askerud, Pernille, (1958), works as freelance consultant to governments and international agencies, though chiefly to UNESCO. She is the author of four handbooks published by UNESCO, most recently Cultural Industries Statistics (2007). She has specialized in research and organizational development, especially in terms of institutional capacity building, and the identification of policies and project design that more adequately respond to socio-economic changes that challenge the effectiveness of established development modalities. Since 2002 she has been lead consultant for the research and implementation of UNESCO's programme activities for cultural industries in Asia-Pacific.

Contact: pernille@ askerud.org

Beyes, Timon, (1973), Dr., Senior Lecturer, University of Sankt Gallen, Switzerland. Research interests include: organizing in nonprofitorganizations; the organizing of space and the spaces of organizing; the aesthetics and politics of urban cultural/artistic interventions. Recent publications: Beyes/Deuflhard (eds.) (2009, forthcoming): ParCITYpate: Artistic Interventions and Urban Space; Beyes (2009): 'Spaces of intensity - urban entrepreneurship as redistribution of the sensible'. In: Hjorth/Steyaert (eds.), The Politics and Aesthetics of Entrepreneurship, Cheltenham: Edward Elgar, pp. 92-112.

Contact: timon.beyes@unisg.ch 
Böhning, Björn, (1978), Politikwissenschaftler, Leiter des Planungsstabs in der Senatskanzlei Berlin. Aktuelle Publikationen: Böhning/Burmeister (2004): Generationen \& Gerechtigkeit, Hamburg: VSA Verlag; Böhning et al. (2006): Unterschichten?, Prekariat?, Klassen? Moderne Politik gegen soziale Ausgrenzung, Dortmund: SPW Verlag. Kontakt: Bjoern.Boehning@senatskanzlei.berlin.de

Bürkner, Hans-Joachim, (1954), Prof. Dr., Professor für Wirtschaftsund Sozialgeographie an der Universität Potsdam, zugl. Senior Researcher am Leibniz-Institut für Regionalentwicklung und Strukturplanung (Erkner bei Berlin). Forschungsfelder: Soziale Milieus, räumlich-soziale Disparitäten, Migration, Wissensökonomie, Kreativwirtschaft, Urban Governance, Place-making. Aktuelle Publikation: Bürkner (2008): „Reaching for the Stars: East German Urban Regions and the Vicissitudes of Placemaking“. In: Scott (Hg.): De-coding New Regionalism: Shifting Socio-Political Contexts in Central Europe and Latin America. London: Ashgate. S. 215-232;

Kontakt: buerkner@irs-net.de

Dresel, Sebastian, (1975), Beauftragter für Musik und Popkultur der Stadt Mannheim, Organisator des „Forums kreative Stadt“ in Mannheim, das im April 2009 zum zweiten Mal stattfand.

Kontakt: Sebastian.Dresel@mannheim.de

Eichmann, Hubert, (1969), Dr., Soziologe. Senior Researcher bei der Forschungs- und Beratungsstelle Arbeitswelt, Wien. Forschungsfelder: Arbeits-, Professions- und Kultursoziologie; Raum- und Stadtentwicklung. Aktuelle Publikationen: Eichmann/Schiffbänker (Hg.) (2008): Nachhaltige Arbeit in der Wiener Kreativwirtschaft? Architektur, Design, Film, Internet, Werbung, Wien (u. a.): Lit-Verlag; Eichmann/ Hofbauer (2008): „Man braucht sehr hohes Energieniveau“: Zum Arbeitsalltag von UnternehmensberaterInnen, Berlin: edition sigma. Kontakt: eichmann@forba.at

Färber, Alexa, (1968), Dr. phil., Wissenschaftliche Mitarbeiterin Seminar für Europäische Ethnologie/Volkskunde Christian-Albrechts-Universität zu Kiel, seit April 2009 Juniorprofessorin für Europäische Ethnologie an der Humboldt-Universität zu Berlin. Forschungsfelder: Stadtanthropologie, Islam im urbanen Raum, Repräsentationsarbeit, Wissensanthropologie, Kulturelle Semantik ethnografischer Verfahren. Aktuelle Publikationen: Althans/Audehm/Binder/Ege/Färber (2008): „Kreativität. Eine Rückrufaktion“. In: Zeitschrift für Kulturwissen- 
schaften 1; Färber (2008): „Flourishing Cultural Production in Economic Wasteland: Three Ways of Making Sense of a Cultural Economy in Berlin at the Beginning of the Twenty-first Century“. In: Heßler/ Zimmermann (Hg.): Creative Urbane Milieus. Historical Perspectives on Culture, Economy, and the City, Frankfurt/Main: Campus, S. 409-428.

Kontakt: alexa.faerber@rz.hu-berlin.de

Förster, Agnes, (1976), Dipl.-Ing. Architektin, wissenschaftliche Mitarbeiterin am Lehrstuhl für Raumentwicklung der Technischen Universität München, Partnerin im Büro 4architekten, München. Forschungsfelder: Einsatz von Analyse, Visualisierung und Kommunikation in der Raumentwicklung sowie Fragen der Wahrnehmung und Identität von Stadtlandschaften. Aktuelle Publikation: Thierstein/Förster (Hg.) (2008): The Image and the Region - Making Mega-City Regions Visible! Baden: Lars Müller Publishers.

Kontakt: foerster@raumentwicklung.ar.tum.de

Günther, Constanze, (1980), M.A. Angewandte Kulturwissenschaften. Mitarbeiterin der IBA Hamburg im Querschnittsprojekt „Kreatives Quartier Elbinsel“ sowie Doktorandin an der Leuphana Universität Lüneburg zum Thema „,Strategische Kreativplanung der Stadt: Die Internationale Bauausstellung (IBA) Hamburg" (Arbeitstitel). Aktuelle Publikation: Günther (2008): „Kreatives Quartier Elbinsel - Handlungsfelder von Kunst und Kultur im Rahmen der IBA Hamburg“. In: Stadtkultur Magazin 04.

Kontakt: constanze.guenther@iba-hamburg.de

Kalandides, Ares, (1965), M.A. Stadt- und Raumplanung. Stadtmarketing und -branding. Geschäftsführer der INPOLIS GmbH und Ph.D. Cand. an der Technischen Universität Athen, Bereich Stadtgeographie. Aktuelle Publikationen: Kalandides (2008): „Kreativwirtschaft und digitale Bohemiens“. In: Garten+Landschaft 7; Kalandides/Lange/Stöber/Mieg (2008): „Berlin's Creative Industries: Governing Creativity?“ In: Industry and Innovation, Vol. 15, No. 5, S. 531-548; Kalandides (2007): „Marketing the Creative Berlin and the Paradox of Place Identity“. Präsentiert in: XXVII Conferenza Scinentifica Annuale dell' Associazione Italiana di Scienze Regionali, Bozen, Italien.

Kontakt: Kalandides@inpolis.de 
Krusche, Bernhard, (1960), Dr., Ethnologe and Geschäftsführer der Beratungsfirma osb Tübingen GmbH, Projektkoordinator der „Next City Graz-Reininghaus“. Forschungsfelder: Governance von komplexen Systemen, Organisationstheorie, Leadership. Aktuelle Publikationen: Krusche (2008): Paradoxien der Führung, Heidelberg: Carl-Auer-Verlag; Krusche (2008): „Houston, we have a Problem“. In: Revue für postheroisches Management, Heft 3, S. 72-81.

Kontakt: Bernhard.Krusche@osb-i.com

Kunzmann, Klaus R., studierte Architektur und Stadtplanung in an der TU München und promovierte 1972 an der TU Wien. Von 1974 bis 2006 war er Professor an der Fakultät Raumplanung der Universität Dortmund. Er ist Honorarprofessor am University College London und an der Chung Hua Universität in Hinchu/Taiwan. Interessen- und Forschungsschwerpunkte ist seit über 20 Jahren die Kulturwirtschaft. Er gehört zu den Autoren der bisher erschienen fünf Kulturwirtschaftsberichte des Landes NRW. Veröffentlichungen zu Themen der Kreativ- und Kulturwirtschaft.

Kontakt: klaus.kunzmann@udo.edu

Lange, Bastian, (1970), Dr. phil, Dipl.-Geograph. Post-Doc Researcher am Leibniz-Institut für Länderkunde Leipzig, Mit-Initiator des Forschungskreises „Governance der Creative Industries“ am GeorgSimmel-Zentrum für Metropolforschung der Humboldt Universität zu Berlin. Forschungsfelder: Creative and Knowledge Industries, Milieuund Szeneforschung, Entrepreneurship, Raumtheorien und Governance. Aktuelle Veröffentlichungen: Lange (2007): Die Räume der Kreativszenen - Culturepreneurs und ihre Orte in Berlin, Bielefeld: transcript Verlag; Lange (2009): „Accessing Markets in Creative Industries - Professionalization and Social-spatial Strategies of Culturepreneurs in Berlin“, Creative Industries Journal 1: 2, S. 115-135.

Kontakt: Bastian.Lange@berlin.de

Leitner-Sidl, Stefan, (1974), Magister der Wirtschafts- und Sozialwissenschaften an der WU Wien, Gründer der UnternehmerInnenzentren Schraubenfabrik, Hutfabrik und Rochuspark in Wien. Themenschwerpunkte: Kreativwirtschaft, Ein-Personen-Unternehmen, CommunityBuilding.

Kontakt: leitner-sidl@konnex.cc 
Lüthi, Stefan, (1978), Dipl.-Geograph. wisenschaftlicher Mitarbeiter am Lehrstuhl für Raumentwicklung der Technischen Universität München. Forschungsfelder: Metropolregionen, Regionale Innovationssysteme, Fragen der Innovations- und Regionalpolitik, quantitative und qualitative Methoden der Netzwerkanalyse. Aktuelle Publikation: Thierstein/Lüthi/Kruse/Gabi/Glanzmann (2008): „The changing value chain of the knowledge economy. Spatial impact of intra-firm and inter-firm networks within the emerging Mega-City Region of Northern Switzerland“. In: Regional Studies, Vol. 42/8, S. 1113-1131.

Kontakt: luethi@raumentwicklung.ar.tum.de

McRobbie, Angela, is Professor of Communications at Goldsmiths College University of London. Her earliest books and publications were written while still a student at the Birmingham University Centre for Contemporary Cultural Studies. Her most recent books include British Fashion Design (Routledge 1998), In the Culture Society (Routledge 1999), The Uses of Cultural Studies (Sage 2005) and the Aftermath of Feminism (Sage 2008). Her work is widely translated, and she is currently completing two books, one on new culture industry titled $\mathrm{Be}$ Creative, the other Post Feminist Art, Image, Music, Text. Angela McRobbie lives in London and Berlin.

Contact: angela.mcrobbie@gold.ac.uk

Mieg, Harald A., Dr., (1961), Umweltsozialwissenschaftler (ETH), HANS-SAUER-Professor für Metropolen- und Innovationsforschung am Geographischen Institut der Humboldt-Universität zu Berlin; Leiter des Georg-Simmel-Zentrums für Metropolenforschung der HU zu Berlin. Aktuelle Publikationen: Mieg (2005): „Professionalisierung“. In: Rauner (Hg.), Handbuch der Berufsbildungsforschung, Bielefeld: Bertelsmann, S. 342-349; Lange/Mieg (2008): „Professionalisierungswege und Konstituierungen von ,Märkten“ in den Creative Industries“. In: Geographische Zeitschrift, 94 (4), S. 225-242.

Kontakt: harald.mieg@env.ethz.ch

Olma, Sebastian, (1973), Dr., Organisationssoziologe und Consultant. Aktuelle Publikationen: Olma (Hg.) (2007): Life's (Re)Emergence: Theory, Culture \& Society Special Issue, London: Sage; Olma (2007): „On the Creativity of the Creative Industries“. In: Lovink/Rossiter (Hg.), My Creavitity Reader, Institute of Network Cultures, Amsterdam, S. 261-267.

Kontakt: sebolma@xs4all.nl 
Ooi, Can-Seng, (1965), PhD, Associate Professor, Copenhagen Business School, Master of Social Science degree in Sociology from the National University of Singapore. Research on Creative Industries, Place Branding, Tourism. Recent publications: Ooi (2008): 'Reimagining Singapore as a creative nation: The politics of place branding'. In: Place Branding and Public Diplomacy, Vol. 4, pp. 287-302; Ooi (2007): 'The creative industries and tourism in Singapore'. In: Richards/Wilson (eds.) Tourism, Creativity and Development, London: Routledge, pp. 240 251.

Contact: co.int@cbs.dk

Pratt, Andy C., (1959), Reader in Urban Cultural Economy, Department of Geography and Environment and Director of Urban Research Centre, London School of Economics. Research on the cultural economy, organisation and space in the international economy; urbanisation, innovation and culture; creative and cultural industries policy. Recent publications: Pratt/Jeffcutt (eds.) (2009): Creativity, Innovation and the Cultural Economy, London: Routledge; Kretschmer/Pratt (2009): 'Music, copyright and information and communications studies'. In: Information, Communication \& Society 12, pp. 1-13.; Gill/Pratt (2008): 'In the social factory? Immaterial labour, precariousness and cultural work'. In: Theory, Culture \& Society 25, pp. 1-30.

Contact: a.c.pratt@1se.ac.uk

Prossek, Achim, (1970), Dr. rer pol, Wissenschaftlicher Mitarbeiter an der Fakultät Raumplanung der Technischen Universität Dortmund. Forschungsfelder: Raumbilder und räumliche Identität, Kultur und Regionalentwicklung. Aktuelle Publikationen: Prossek (2009): Bild-Raum Ruhrgebiet. Zur symbolischen Produktion der Region, Dortmund: Rohn; Prossek (2008): „Visuelle Regionsproduktion. Ruhrgebiet im Blick“. In: Zeitschrift für Kulturwissenschaften: Räume, Heft 2/2008, S. 65-75;

Kontakt: achim.prossek@tu-dortmund.de

Seng, Judith, (1974), Dipl. Designerin, Vertretungsprofessorin für Prozesse und Methoden des Entwerfens an der Kunsthochschule Kassel. Zuletzt leitete sie die Prozess- und Designentwicklung sowie die Publikation im Design Reaktor Berlin. Ihr Arbeitsfeld umfasst die Gestaltung von Prozessen und Studien, aber auch von Objekten und Räumen. Ihre Arbeiten werden international ausgestellt. Aktuelle Publikation: Kufus/Piesbergen/Schirrmacher/Seng (2008), Design Reaktor Berlin, Verlag der Universität der Künste Berlin.

Kontakt: mail@judithseng.de 
Sikiaridi, Elizabeth and Frans Vogelaar are founders of Hybrid Space Lab (Amsterdam/Berlin), an $R \& D$ and design practice focusing on the hybrid fields that are emerging through the combination and fusion of environments, objects and services in the information/communication age. In 1998 Frans founded at the Academy of Media Arts Cologne the first Department of Hybrid Space worldwide. Elizabeth was appointed professor at the University of Duisburg-Essen in 1997, working on the design and development of the cityscape. Elizabeth and Frans have worked as a consultant to the Dutch government on 'the use of space in the information/communication age'.

Contact: office@hybridspacelab.net

Steyaert, Chris, (1962), Doctor in Psychology. Professor for Organizational Psychology, University of Sankt Gallen. Research themes: Narrating (Urban) Entrepreneurship, Multilingualism in International Organizations and Creative, Reflexive and Diverse Organizing. Recent publications: Hjorth/Steyaert (eds.) (2009), The Politics and Aesthetics of Entrepreneurship, Cheltenham: Edward Elgar; Chris Steyaert, 'Entrepreneuring as a conceptual attractor?: A review of process theories in 20 years of entrepreneurship studies?' In: Entrepreneurship and Regional Development, 2007, 19 (6), pp. 453-477.

Kontakt: chris.steyaert@unisg.ch

Stöber, Birgit, (1969), PhD, Associate Professor in Cultural Geography, Copenhagen Business School. Mit-Initiatorin des Forschungskreises „Governance der Creative Industries“ am Georg-Simmel-Zentrum für Metropolforschung der Humboldt Universität zu Berlin. Forschungsfelder: Creative Industries, Place Branding, Governance, Media Studies. Aktuelle Publikationen: Lange/Kalandides/Stöber/Mieg (2008): „Berlin's Creative Industries: Governing Creativity?“ In: Industry and Innovation 15, Vol. 5, S. 531-548; Stöber (2008): „Place Branding - How the Private creates the Public“. In: Hansen/Salskov-Iversen (Hg.) (2008): Critical Perspectives on Private Authority in Global Politics. Basingstoke: Palgrave Macmillan, S. 169-187.

Kontakt: bst.ikl@cbs.dk

Thierstein, Alain, (1957), Prof. Dr. oec., Technische Universität München: Lehrstuhl für Raumentwicklung; Forschungsfelder: Regional- und Stadtentwicklung, insbesondere wissensbasierte Unternehmenstätigkeiten, Metropolitan- und Raumentwicklungspolitik sowie Evaluation von Politikmaßnahmen. Aktuelle Publikationen: Thierstein/Schein (2008): „Emerging Cities on the Arabian Peninsula: Urban Space in the Knowl- 
edge Economy Context“. In: International Journal of Architectural Research, 2 (2), S. 178-195; Thierstein/Lüthi/Kruse/Gabi/Glanzmann (2008): „The changing value chain of the knowledge economy. Spatial impact of intra-firm and inter-firm networks within the emerging MegaCity Region of Northern Switzerland“. In: Regional Studies, 42/8, S. 1113-1131.

Kontakt: thierstein@ raumentwicklung.ar.tum.de

Wellmann, Inga, (1977), Dipl. Kulturmanagerin, BA Mixed Media Artist. Geschäftsführerin des Einstein Forum in Potsdam; Vorstandsmitglied von berlinpolis e. V. Inga Wellmann arbeitet seit vielen Jahren an der Schnittstelle von Kunst, Wirtschaft und Wissenschaft und untersucht in diesem Zuge u. a. die Rolle kreativer Intermediäre in Transformationskontexten. Sie konzipiert und organisiert Veranstaltungen im Bereich der Kreativwirtschaft und berät zu diesem Thema Akteure der Politik, Kultur und Wirtschaft. Aktuelle Publikation: Wellmann (2009): „Sozialstatik und Schnittstellengestaltung. Was sich in Krisenzeiten von den kreativen Ökonomien lernen lässt“. In: Dettling/Schüle (Hg): Minima Moralia der nächsten Gesellschaft, Berlin: VS-Verlag für Sozialwissenschaften.

Kontakt: inga.wellmann@einsteinforum.de 


\section{»Der Mittelzeeg 36 ist in der kritischen Gesellschafts- wissenschaft mittlerweile zu einer Institution geworden." Süddeutsche Zeitung}
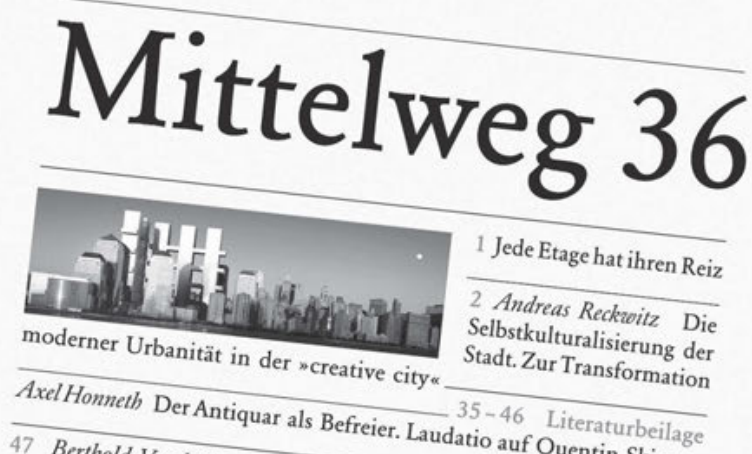

47 Berthold Vogel Streit, diagnostik?
donflikttheorie zu einer akteursorientierten Gesellschafts-
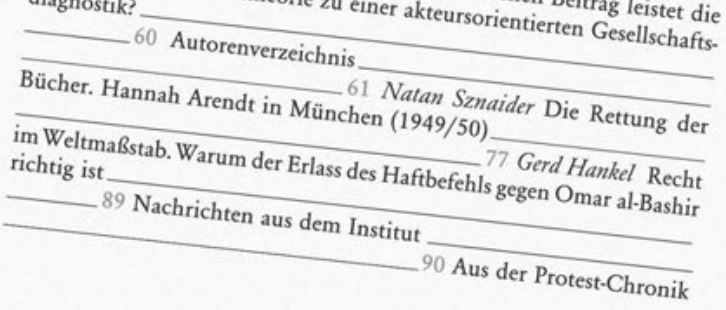

Bestellen Sie unser Probeabonnement (3 Ausgaben in Folge) für nur € 20,inkl. Versand (ohne automatische Verlängerung): Redaktion Mittelweg 36, Hamburger Institut für Sozialforschung, Mittelweg 36, 20148 Hamburg, Tel. 040/414097-0, E-Mail: zeitschrift@mittelweg 36.de www.mittelweg36.de 


\section{Urban Studies}

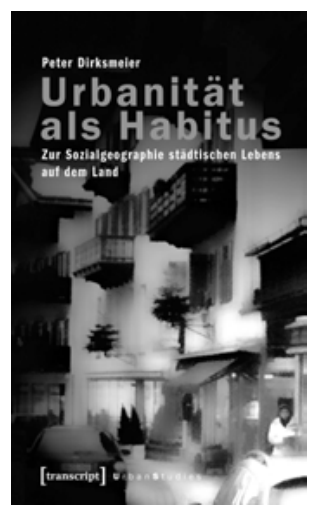

Peter Dirksmeier

Urbanität als Habitus

Zur Sozialgeographie städtischen

Lebens auf dem Land

April 2009, 296 Seiten, kart., 28,80€, ISBN 978-3-8376-II27-4

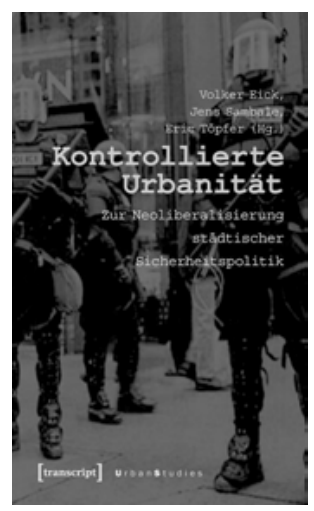

Volker Eick, Jens Sambale, ERIC TÖPfER (HG.)

Kontrollierte Urbanität

Zur Neoliberalisierung städtischer Sicherheitspolitik

2007, 402 Seiten, kart., 21,00 €, ISBN 978-3-89942-676-2

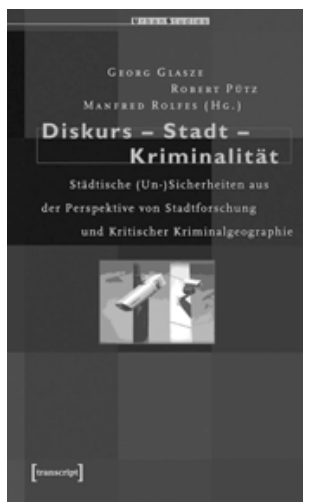

Georg Glasze, Robert Pütz, Manfred Rolfes (Hg.)

Diskurs - Stadt - Kriminalität Städtische (Un-)Sicherheiten aus der Perspektive von Stadtforschung und Kritischer Kriminalgeographie

2005, 326 Seiten, kart., $27,80 €$, ISBN 978-3-89942-408-9

Leseproben, weitere Informationen und Bestellmöglichkeiten finden Sie unter www.transcript-verlag.de 


\section{Urban Studies}
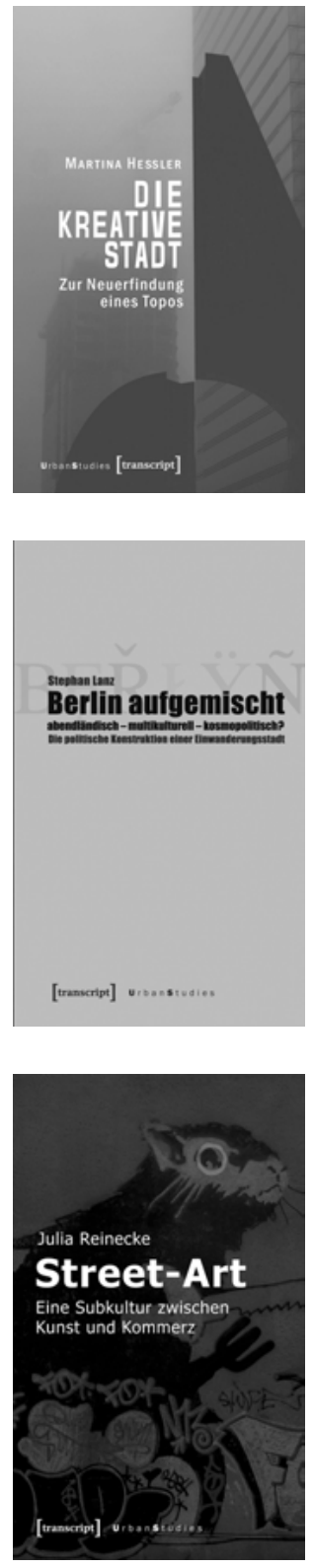

Martina Hessler

Die kreative Stadt

Zur Neuerfindung eines Topos

2007, 364 Seiten, kart., zahlr. Abb., 29,80€, ISBN 978-3-89942-725-7

\section{StEPHAN LANZ}

Berlin aufgemischt: abendländisch, multikulturell, kosmopolitisch? Die politische Konstruktion einer Einwanderungsstadt

2007, 434 Seiten, kart., 34,80€, ISBN 978-3-89942-789-9

\section{Julia ReineCKe}

Street-Art

Eine Subkultur zwischen Kunst und Kommerz

2007, I94 Seiten, kart., zahlr. farb. Abb., 23,80€, ISBN 978-3-89942-759-2 


\section{Urban Studies}

ULRIKE GERHARD

Global City Washington, D.C.

Eine politische Stadtgeographie 2007, 304 Seiten, kart., 29,80€, ISBN 978-3-89942-497-3

Katrin Grossman N

Am Ende des Wachstumsparadigmas?

Zum Wandel von

Deutungsmustern in

der Stadtentwicklung.

Der Fall Chemnitz

2007, 272 Seiten, kart., zahlr. Abb., $26,80 €$,

ISBN 978-3-89942-7I8-9

Nicole Grothe

\section{InnenStadtAktion -}

Kunst oder Politik?

Künstlerische Praxis

in der neoliberalen Stadt

2005, 282 Seiten, kart., zahlr. Abb.,

$25,80 €$,

ISBN 978-3-89942-4I3-3

Simon GÜNTneR

Soziale Stadtpolitik

Institutionen, Netzwerke und

Diskurse in der Politikgestaltung

2007, 406 Seiten, kart., 35,80€,

ISBN 978-3-89942-622-9

Susanne Heeg

Von Stadtplanung und

Immobilienwirtschaft

Die »South Boston Waterfront« als Beispiel für eine neue

Strategie städtischer Baupolitik 2008, 276 Seiten, kart., 26,80€, ISBN 978-3-89942-819-3
Andrej Holm

Die Restrukturierung

des Raumes

Stadterneuerung der goer Jahre in Ostberlin: Interessen und Machtverhältnisse 2006, 356 Seiten, kart., 29,80€, ISBN 978-3-89942-52I-5

UWE LEWITZKY

Kunst für alle?

Kunst im öffentlichen Raum zwischen Partizipation,

Intervention und

Neuer Urbanität

2005, I3 8 Seiten, kart., I4,80€,

ISBN 978-3-89942-285-6

Annika Mattissek

Die neoliberale Stadt

Diskursive Repräsentationen

im Stadtmarketing deutscher

Großstädte

2008, 298 Seiten, kart., $28,80 €$,

ISBN 978-3-8376-Io96-3

Thomas Pohl

Entgrenzte Stadt

Räumliche Fragmentierung und zeitliche Flexibilisierung in der Spätmoderne

April 2009, 394 Seiten, kart., zahlr. z.T. farb. Abb., 29,80€, ISBN 978-3-8376-III8-2

\section{Sonia Schoon}

Shanghai XXL

Alltag und Identitätsfindung im Spannungsfeld extremer Urbanisierung

2007, 344 Seiten, kart., 32,80€, ISBN 978-3-89942-645-8 